hep-th/0007195

\title{
Black Hole Entropy, Special Geometry and Strings
}

\author{
Thomas Mohaupt田 \\ Martin-Luther-Universität Halle-Wittenberg, Fachbereich Physik, D-06099 Halle, Germany
}

\begin{abstract}
We review work done over the last years on the macroscopic and microscopic entropy of supersymmetric black holes in fourdimensional $\mathcal{N}=2$ supergravity and in $\mathcal{N}=2$ compactifications of string theory and M-theory. Particular emphasis is put on the crucial role of higher curvature terms and of modifications of the area law in obtaining agreement between the macroscopic entropy, which is a geometric property of black hole solutions and the microscopic entropy, which is computed by state counting in Calabi-Yau compactifications of string or M-theory. We also discuss invariance properties of the entropy under stringy T-duality and S-duality transformations in $\mathcal{N}=2,4$ compactifications in presence of higher curvature terms.

In order to make the paper self-contained we review the laws of black hole mechanics in higher derivative gravity, the definition of entropy as a surface charge, the superconformal offshell description of $\mathcal{N}=2$ supergravity, special geometry, and $\mathcal{N}=2$ compactifications of heterotic and type II string theory and of M-theory.
\end{abstract}

PACS numbers: 04.65+e, 04.70. s, 11.25. w, 11.25.Mj

Key words: Supergravity, Black Holes, String theory, Compactification December 1999 / September 2000

\footnotetext{
1 mohaupt@hera1.physik.uni-halle.de
} 


\section{Contents}

1 Introduction and Overview 5

1.1 Introduction . . . . . . . . . . . . . . . . . . 5

1.2 Overview . . . . . . . . . . . . . . . . . . . . . 6

2 Gravity, Black Holes and Supersymmetry $\quad 9$

2.1 Gravity, Strings and Effective Field Theories . . . . . . . . . . . . . . . . . . . . 9 9

2.2 Black Holes . . . . . . . . . . . . . . . . . . . . . . . . . . . . . 12

2.2 .1 Basic Properties of Black Holes . . . . . . . . . . . . . . . . . . . . . . . . 12

2.2 .2 The Laws of Black Hole Mechanics . . . . . . . . . . . . . . . . . . . . . . 13

2.2.3 Quantum Aspects of Black Holes and Black Hole Thermodynamics . . . 14

2.2 .4 Black Hole Horizons . . . . . . . . . . . . . . . . . . . . 16

2.2 .5 Surface Charges $\quad$. . . . . . . . . . . . . . . . . . . . . . . . . . . . . . . 18

2.2 .6 The First Law in Higher Derivative Gravity . . . . . . . . . . . . . . . . . 19

$2.2 .7 \quad$ Extremal Black Holes as Supersymmetric Solitons . . . . . . . . . . . . . 23

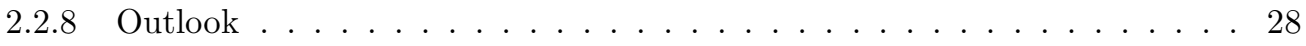

3 Four-Dimensional $\mathcal{N}=2$ Supergravity 29

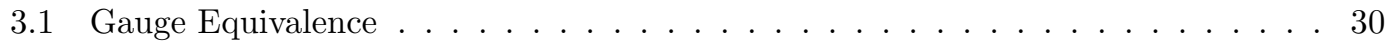

3.2 Superconformal Multiplets . . . . . . . . . . . . . . . . . . . . . . . . . 32

3.2.1 The Superconformal Algebra . . . . . . . . . . . . . . . . . . . . . . 32

3.2 .2 The Weyl Multiplet . . . . . . . . . . . . . . . . . . . . . . . . . . . 34

$3.2 .3 \quad$ Vector Multiplets . . . . . . . . . . . . . . . . . . . . . . . . . . . 39

3.2 .4 Chiral Multiplets . . . . . . . . . . . . . . . . . . . . . . . . 40

3.2.5 The Non-linear Multiplet . . . . . . . . . . . . . . . . . . . . . . 41

3.3 Superconformal Actions . . . . . . . . . . . . . . . . . . . . . . . . . . 43

3.4 Symplectic Reparametrizations . . . . . . . . . . . . . . . . . . . . . . . . . 46

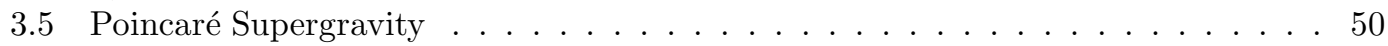

3.5.1 Poincaré Gauge Fixing $\quad$. . . . . . . . . . . . . . . . . . . . . . . 50

3.5.2 Special Geometry in Special Coordinates . . . . . . . . . . . . . . . . . 54

3.5.3 Special Geometry in General Coordinates . . . . . . . . . . . . . . . . . . 57

3.5.4 Consequences of the Presence of Higher Derivative Terms . . . . . . . . . 61

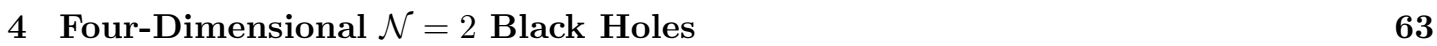

4.1 The Near Horizon Geometry 1 . . . . . . . . . . . . . . . . . . . . . . . . . . . . 64

4.1.1 The Gaugino Variations . . . . . . . . . . . . . . . . . . . . . . . . 65

4.1 .2 The Background Spinor Variation . . . . . . . . . . . . . . . . . . . . . 66

4.1.3 The Gravitini Variations . . . . . . . . . . . . . . . . . . . . . . . . . . . . 66

$4.1 .4 \quad$ Variation of the Spinor of the Second Compensating Multiplet . . . . . . 68

$4.1 .5 \quad$ Variation of the Derivative of the Spinor $\zeta_{i} \ldots \ldots . \ldots$. . . . . . . 69

4.1.6 The Relation between the Auxiliary T-Field and the Gauge Fields . . . . 69

4.1 .7 The Gauge Field Equations of Motion . . . . . . . . . . . . . . . . . . . 70

4.1 .8 The Metric . . . . . . . . . . . . . . . . . . . . 71

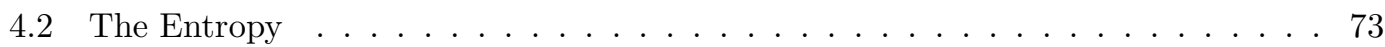


4.2 .1 The Entropy Formula _ . . . . . . . . . . . . . . . . . . . . . . . 73

4.2 .2 The Stabilization Equations . . . . . . . . . . . . . . . . . . . . . 74

4.3 Black hole Solutions $\quad \ldots \ldots \ldots \ldots$. . . . . . . . . . . . . . . 76

$4.4 \quad$ More recent results on stationary BPS solutions in presence of $R^{2}$-terms . . . . 80

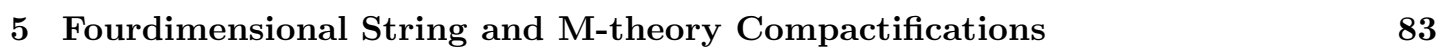

5.1 Type II String Theory on a Calabi-Yau Threefold . . . . . . . . . . . . . . . . . . 83

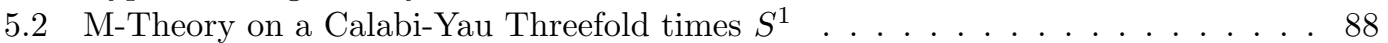

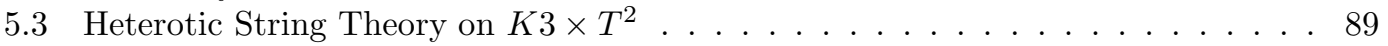

5.3.1 The Tree Level Prepotentiall. . . . . . . . . . . . . . . . . . . . . . . . . . 90

5.3 .2 The Perturbative Heterotic Prepotential . . . . . . . . . . . . . . . . . . . 93

5.3 .3 The Non-Perturbative Heterotic Prepotential . . . . . . . . . . . . . . . . 96

$5.3 .4 \quad$ Higher Derivative Couplings in Heterotic Compactifications . . . . . . . . 99

5.3 .5 Compactifications with $\mathcal{N}=4$ Supersymmetry . . . . . . . . . . . . . 100

$6 \quad$ Four-Dimensional $\mathcal{N}=2$ Black Holes in String and M-Theory 103

6.1 The Macroscopic Black Hole Entropy . . . . . . . . . . . . . . . . . . . . . . . . 104

6.1.1 Black Holes in Type II String Theory and in M-Theory . . . . . . . . . . 104

6.1 .2 Black Holes in Heterotic String Theory . . . . . . . . . . . . . . . . . . . 108

6.2 The Microscopic Black Hole Entropy

6.2 .1 Computation of the Microscopic Black Hole Entropy . . . . . . . . . . . . 115

6.2.2 Comparison of Macroscopic and Microscopic Results . . . . . . . . . . . 119

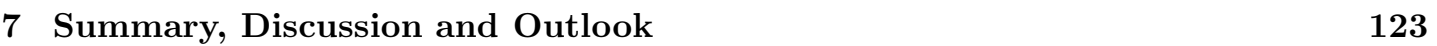

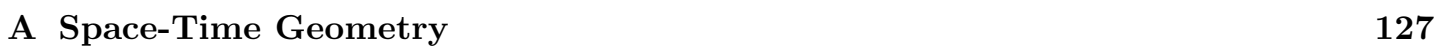

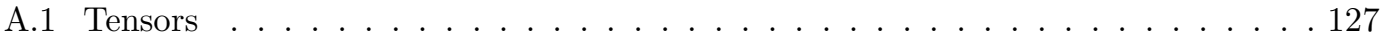

A.1.1 Metric and Vielbein . . . . . . . . . . . . . . . . . . . . . 127

A.1.2 Symmetrization and Antisymmetrization . . . . . . . . . . . . . . . . . . 127

A.1.3 $\quad$ Selfdual and Antiselfdual Antisymmetric Tensors . . . . . . . . . . . . . . 128

A.1.4 Spin Connection and Riemann Tensor . . . . . . . . . . . . . . . . . . . . 128

A.1.5 Consequences of Rotational Invariance . . . . . . . . . . . . . . . . . . . . 129

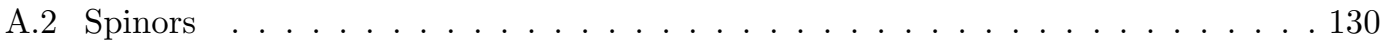

A.2.1 $\gamma$-Matrices . . . . . . . . . . . . . . . . . . 130

A.2.2 Charge Conjugation, Dirac-, Majorana- and Weyl-Spinors . . . . . . . . . 131

A.2.3 Spinor Bilinears and Fierz Rearrangements . . . . . . . . . . . . . . . . . 131

\begin{tabular}{|l|l}
\hline B Abelian Gauge Fields & 133
\end{tabular}

B.1 Maxwell and Einstein-Maxwell Theory . . . . . . . . . . . . . . . . . . . . . . . . 133

B.2 Abelian Gauge Fields coupled to $\mathcal{N}=2$ Supergravity . . . . . . . . . . . . . . . . 136

\begin{tabular}{|r|r|}
\hline C Covariant Derivatives & 139
\end{tabular}

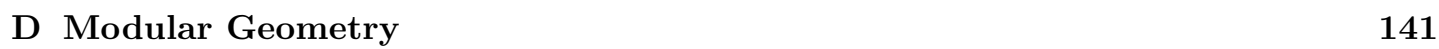

E The Polylogarithmic Functions 145 


\section{Chapter 1}

\section{Introduction and Overview}

\section{$1.1 \quad$ Introduction}

Black holes are truly unique objects: For theoretical physicists they pose various fascinating problems, which may offer a clue for solving the riddles of quantum gravity. At the same time black holes are an established part of pop culture. And even more astonishing it is very likely that black holes really exist beyond imagination: Currently about 20 stellar binaries are known in our galaxy which are believed to contain black holes of some solar masses, whereas supermassive black holes provide the only explanation for the processes observed in the centers of active galaxies [1]. The gravitational wave detectors GEO 600 [2], VIRGO [3] and LIGO [4], which are currently under construction, aim to directly observe processes involving black holes, including collisions of black holes, in our cosmic neighbourhood of about $25 \mathrm{Mpc}$.

In this paper we will consider black holes from the theoretical perspective. The most interesting questions of black hole physics are related to one of the fundamental problems of contemporary physics, namely to find a synthesis of the ideas of dynamical space-time geometry and of quantum physics. This has nourished the hope that black holes might turn out to be 'the hydrogen atom of quantum gravity'. Though it remains to be seen whether this is finally true, tremendous progress on several aspects of black holes has been made during the last four years using string theory. String theory [5, 6, 7] is the leading candidate for a unified quantum theory of elementary particles and their interactions, including gravity. During the 'first string revolution' of the mid-eighties it became clear that string theory is consistent at the level of perturbation theory. This was an important step, but left non-perturbative problems, such as the study of black holes, out of reach. The 'second string revolution' of the mid-nineties has changed this dramatically. The discovery of string dualities led to a new picture, where the strong coupling limit of every string theory is described by a weakly coupled dual theory, which is either a string theory, or eleven-dimensional supergravity. Moreover all five superstring theories and eleven-dimensional supergravity seem to be asymptotic expansions of one single underlying theory, called M-theory. A central role in establishing string dualities has been played by solitons, and in particular by $p$-branes, which are higher dimensional analogues of black holes. Conversely string theory can now be used to investigate problems of black hole physics. One approach is to interpret black holes and string excitations as dual descriptions of the same object 8, 9]. This so-called string - black hole correpondence passes some qualitative tests: it predicts the black hole entropy in terms of string states up to a constant of order unity and it gives an explanation for the final state of a Schwarzschild black hole: It heats up until it reaches the Planck temperature and then converts into a highly excited string state.

More precise and quantitative tests are possible for the subclass of charged extremal black holes. When embedded into theories with extended supersymmetry, extremal black holes are BPS solitons [10]. Since the essential properties of such objects are determined by supersymmetry, it is possible to do computations in the perturbative regime and then to extrapolate them to finite coupling. This became a powerful tool after the discovery that charged black 
holes in string theory have a dual, perturbative description in terms of D-branes 11. Fourand five-dimensional extremal black holes can be embedded into string theory. From the point of view of ten-dimensional string theory or eleven-dimensional M-theory extremal black holes are bound states of $p$-branes and other gravitational solitons. Using the BPS property one can study these brane configurations in the perturbative regime. In particular one can count the microstates of a black hole, which means all states of the brane configuration which lead to the same four- or five-dimensional black hole, and one can compute the corresponding 'microscopic' or 'statistical' entropy. Since one can independently compute the 'macroscopic' entropy of the black hole, which means its Bekenstein-Hawking entropy, the comparison of the two results provides a test both for the hypothesis that the Bekenstein-Hawking entropy really is an entropy, and for the microscopic model of black holes provided by D-branes. Using this strategy Strominger and Vafa showed that the two entropies agree quantitatively for certain classes of black holes in string compactifications [12]. Here 'quantitatively' means that the leading terms for both entropies are exactly the same. The D-brane method has also been applied to derive Hawking radiation and to compute greybody factors. In addition one gets a resolution of the information or unitarity problem of quantum gravity: According to the D-brane picture the time-evolution of a black hole is unitary, and the Planckian character of Hawking radiation is due to averaging over initial and summing over final states. We refer to [13] for a review and further references and to [14] for an elementary introduction to black holes in supergravity and string theory.

In this paper we will focus on black hole entropy. The original work of Strominger and Vafa studied five-dimensional black holes in compactifications with $\mathcal{N}=4,8$ supersymmetry. Of course, one is more interested in four-dimensional black holes and the method applies whenever extremal black holes are BPS states. This means that the most general setting is black holes in compactifications with $\mathcal{N}=2$ supersymmetry. It turns out that the generalization to this case is highly non-trivial. The reason is that four-dimensional supergravity theories with 8 supercharges $(\mathcal{N}=2$ in four dimensions) have a much richer dynamics than theories with 16 or 32 supercharges $(\mathcal{N}=4,8$ in four dimensions). Whereas the low energy Lagrangian (with terms up to two derivatives) is uniquely fixed by the spectrum in $\mathcal{N} \geq 4$ theories, the low energy Lagrangian of $\mathcal{N}=2$ supergravity contains arbitrary functions. This leaves room for both quantum and stringy corrections, which turn out to be very complicated. Fortunately the Lagrangian is still much more restricted than in theories with $\mathcal{N}=1$ supersymmetry or in non-supersymmetric theories. This makes it possible to keep the theory under sufficient control in order to solve various problems exactly. The relevant sector for black hole solutions consists of the supergravity multiplet together with a model-dependent number of vector multiplets. It is uniquely specified by a single function of the scalar fields, the prepotential, which is restricted to be holomorphic and to be homogenous of degree two. The related geometry of the scalar sector, called special geometry, is a very powerful tool in investigating the theory [15, 16, 17].

When comparing the macroscopic entropy of four-dimensional $\mathcal{N}=2$ black holes to the microscopic entropy computed in the brane picture one encounters subtleties that have not been noticed before. The microscopic entropy formula contains a subleading term, which on the macroscopic side corresponds to the presence of a non-minimal higher curvature term in the effective Lagrangian. Thus one is forced to include such higher curvature terms in the construction of black hole solutions and in the computation of the macroscopic entropy. This is a difficult technical problem, which can be overcome by using the superconformal off-shell formulation of $\mathcal{N}=2$ supergravity, as we will see. A second subtlety is that in the presence of higher curvature terms the entropy formula itself has to be modified and is no longer given by the Bekenstein-Hawking area law [18]. It is the purpose of this paper to explain all these points in detail, to put them into perspective and to review the background material that is needed to understand them.

\subsection{Overview}

Let us now give an overview of the following chapters. 
In chapter 2 we introduce our basic topics. We start with Einstein gravity and then discuss higher derivative gravity. Gravity does not lead to a renormalizable and unitary perturbative quantum field theory and therefore we embed it into string theory. Within string theory one can compute a low energy effective action which contains besides the Einstein-Hilbert term also higher curvature terms. These terms are generated by both quantum corrections and stringy $\alpha^{\prime}$-corrections. After recalling basic properties of black holes we review the four laws of black hole mechanics in the context of Einstein gravity. Then we discuss quantum aspects of black holes and explain why the structural equivalence between the laws of black hole mechanics and the laws of thermodynamics is expected to be more than a formal analogy. Special attention is paid to the problem of black hole entropy and we explain what we mean by macroscopic and microscopic black hole entropy. Then we turn to a more technical discussion of black hole horizons and surface charges. This enables us to understand why the laws of black hole mechanics, which were originally derived in Einstein gravity, are independent of the precise form of the equations of motion and also apply, with some subtle modifications, to higher derivative gravity. Particular emphasis is put on the first law and the definition of black hole entropy given by Wald. Finally we discuss extremal black holes and their interpretation as supersymmetric solitons and BPS states in extended supergravity.

Chapter 3 is devoted to a review of $\mathcal{N}=2$ supergravity in the superconformal off-shell formulation. After explaining the concept of gauge equivalence in a non-supersymmetric example, we present the relevant superconformal off-shell multiplets and the construction of the superconformal action for an arbitrary number of vector multiplets. The action depends on the prepotential, which is a holomorphic and homogenous function of the scalar fields, but is arbitrary otherwise. Particular emphasis is put on the Weyl multiplet and its use in describing a particular class of higher curvature terms, called $R^{2}$-terms in the following. The central concept of symplectic invariance, which generalizes electric - magnetic duality is explained in this setting. Then we describe how the superconformal theory and the gauge-equivalent super Poincaré theory are related. Special geometry is discussed both in terms of special and of general coordinates. We explain the relation between the superconformal off-shell and the geometric on-shell formulation of $\mathcal{N}=2$ supergravity in order to make contact with the majority of the recent literature.

In chapter 10 we study model-independent aspects of black holes in $\mathcal{N}=2$ supergravity with vector multiplets in presence of $R^{2}$-terms. We prove that the only static and spherically symmetric $\mathcal{N}=2$ vacuum besides four-dimensional Minkowski space is the space $A d S^{2} \times S^{2}$. We show that the metric and all other fields for such a vacuum can be expressed in terms of a single quantity $Z$, which is closely related to the central charge of the supersymmetry algebra. Since the $A d S^{2} \times S^{2}$ vacuum is the near horizon geometry of an extremal black hole, we can use the generalized entropy formula of Wald to derive a model-independent expression for the entropy, which is manifestly covariant under symplectic transformations. Then we review the supersymmetric attractor mechanism and argue that it generalizes to the case of $R^{2}$-terms. This implies a set of algebraic equations, called stabilization equations, which determine the entropy in terms of the electric and magnetic charges carried by the black hole. All the results mentioned so far can be obtained without explicitly knowing the black hole solution away from the horizon. For completeness we review what is known about explicit black hole solutions in the case without $R^{2}$-terms and we briefly indicate how these can be included. While preparing this paper for publication, we obtained important new results [19], which are briefly summarized in section 4.4 .

Up to this point the discussion is model independent and does not make use of string theory. The only input is $\mathcal{N}=2$ supergravity and all results apply to general prepotentials. Now we bring string theory into the game in order to have a microscopic model for black holes, where one can identify and count microstates. The embedding of supergravity into string theory also provides us with concrete models. In particular the prepotential and the couplings of higher curvature terms can be computed in string perturbation theory. In chapter 0 we review how fourdimensional $\mathcal{N}=2$ supergravity arises from compactifying type II string theory on a Calabi-Yau threefold, eleven-dimensional M-theory on a Calabi-Yau threefold times a circle and heterotic 
or type I string theory on a $K 3$ surface times a two-torus. The structure of the corresponding prepotentials is explained. In particular we discuss in each case the role of quantum and $\alpha^{\prime}$-corrections. The same is done for the field-dependent couplings of the higher curvature terms. We also review the non-perturbative dualities relating the three compactifications and the role of T-duality in perturbative heterotic compactifications. Finally we briefly discuss compactifications with $\mathcal{N}=4$ supersymmetry.

In chapter 6 we consider the entropy of black holes in four-dimensional $\mathcal{N}=2$ string compactifications both from the macroscopic and microscopic point of view. The macroscopic entropy is computed by solving the stabilization equations and plugging the result into the model-independent entropy formula. We show that the entropy is a series in even powers in the charges, with coefficients related to the higher curvature couplings. Then we specifically discuss black holes in type II and M-theory compactifications and describe three classes of black holes where one can completely solve the stabilization equations and obtain explicit formulae for the entropy in terms of the charges. The most important case is the formula for the entropy of black holes in a large volume Calabi-Yau compactification. This is the most general case where also the microscopic entropy has been computed. Then we turn to black holes in heterotic compactifications and derive entropy formulae which are manifestly invariant under T-duality. As a further application we find the S- and T-duality invariant entropy formula for black holes in $\mathcal{N}=4$ compactifications with $R^{2}$-terms. In the second part of the chapter we review the derivation of the microscopic entropy for black holes in compactifications in $\mathcal{N}=2,4,8$ supersymmetry. For $\mathcal{N}=2$ and $\mathcal{N}=4$ we find subleading terms which are related to $R^{2}$-terms on the macroscopic side. The matching of macroscopic and microscopic entropy is discussed in detail. We explain why it is a highly non-trivial result and recall all the subtleties involved in the matching.

In the final chapter 7 we list the key results on black hole entropy in $\mathcal{N}=2$ supergravity and string compactifications and we give an outlook on further directions of research.

In the five appendices we specify our conventions and collect several useful formulae. 


\section{Chapter 2}

\section{Gravity, Black Holes and Supersymmetry}

In this chapter we introduce our topics: gravity and the role of higher curvature terms in it, string theory and its description by a low energy effective action, the laws of black hole mechanics and the definition of black hole entropy, and last but not least the characterization of extremal black holes as supersymmetric solitons.

\subsection{Gravity, Strings and Effective Field Theories}

Gravity, as a classical theory, is described by Einstein gravity [20, 21, 22], which is based on the Einstein-Hilbert action

$$
S=-\frac{1}{2 \kappa^{2}} \int d^{4} x \sqrt{-g} R .
$$

The coupling of matter to gravity is obtained by minimal substitution, i.e. by replacing the partial derivatives occuring in the matter action by covariant derivatives with respect to the Christoffel and spin connections. The coupling constant $\kappa$ is related to Newtons constant $G_{N}$ by $\kappa^{2}=8 \pi G_{N}$, in natural units where $\hbar=c=1$. In these units the Planck length and mass are $G_{N}=l_{\text {Planck }}^{2}=m_{\text {Planck }}^{-2}$. In the following we will mostly use Planckian units, where one sets in addition $G_{N}=1$. When dimensional analysis is required we can easily reconstruct it. We work in four space-time dimensions and take the metric to have signature $(-+++)$.

Nowadays the only observational facts that might be in contradiction with the above minimal version of Einstein gravity are indications for a very small cosmological constant [23]. At the classical level this is a minor modification of the theory, but within a quantum theory a vanishing or very small cosmological constant constitutes a naturalness problem, see 24] for a review. This is an important topic in quantum gravity, but since it has no direct importance for the study of black hole entropy we will set the cosmological constant to zero throughout the text.

Despite that there is no known fact that cannot be covered by Einstein gravity (possibly extended to contain a cosmological constant) we believe that Einstein gravity cannot be a fundamental theory, because it is conceptually incompatible with the other cornerstone of physics, quantum theory. Therefore a central problem of theoretical physics consists in finding a theory that encompasses both gravity and quantum theory. One option is to quantize Einstein gravity. At the perturbative level Einstein gravity is non-renormalizable 25, 26, 27. It seems to be possible that quantum gravity nevertheless exists non-perturbatively, though one cannot get the appropriate classical limit, namely Einstein gravity [28].1. The most prominent candidate for a quantum theory of gravity is string theory [5, 6, 6]. At the perturbative level string theory

\footnotetext{
${ }^{1}$ See this paper for a review and references on the background independent approach to quantum gravity and on recent attempts to make contact with the string theory approach.
} 
is defined by quantizing the relativistic string in a fixed background geometry (and other classical background fields). It has been shown that five consistent string theories exist, which all are supersymmetric and ten-dimensional, in the sense that ten-dimensional Minkowski space is their most symmetric ground state. String theories give a consistent description of quantum gravity in perturbation theory, i.e. one can compute loop corrections involving gravitons. At the non-perturbative level no explicit and in particular no background independent definition is known. During the last years, however, there has been tremendous progress in understanding non-perturbative properties by studying solitons, instantons and string dualities, see [7] for an overview and references. Such dualities allow one to consistently interpret the strong coupling behaviour of a string theory in terms of the weak coupling behaviour of another, dual theory. This has led to a new picture, where all consistent perturbative string theories arise as asymptotic expansions of one single underlying theory, called M-theory. This theory has another vacuum, eleven-dimensional Minkowski space, where it is described at low energies by eleven-dimensional supergravity.

Among all possible covariant Lagrangians the Einstein-Hilbert Lagrangian is singled out by minimality, in the sense that it only contains terms with up to two derivatives. If this requirement is relaxed, one can write down more general covariant Lagrangians which then contain higher order curvature terms. The theory obtained at the four derivative level is called $R^{2}$-gravity because it contains terms quadratic in the curvature ? $^{2}$

$$
S=\int d^{4} x \sqrt{-g}\left(-\frac{1}{2 \kappa^{2}} R+a R^{2}+b R_{\mu \nu} R^{\mu \nu}+c R_{\mu \nu \rho \sigma} R^{\mu \nu \rho \sigma}+d \square R\right)
$$

(we neglected the cosmological constant). The last term is a total derivative and the same is true for the Gauss-Bonnet combination

$$
\begin{aligned}
\mathrm{GB} & =\frac{1}{2} \epsilon^{\mu \nu \alpha \beta} \epsilon^{\rho \sigma \kappa \omega} R_{\mu \nu \rho \sigma} R_{\kappa \omega \alpha \beta} \\
& =R_{\mu \nu \alpha \beta} R^{\mu \nu \alpha \beta}-4 R_{\mu \nu} R^{\mu \nu}+R^{2} .
\end{aligned}
$$

$R^{2}$-gravity is multiplicatively renormalizable, but since it is non-unitary it does not seem to provide an alternative root to perturbative quantum gravity (see 29] for a review and references). The non-unitarity is related to the fact that the higher curvature terms introduce new degrees of freedom, which lead to negative norm states that cannot be eliminated. The conformally invariant part of $R^{2}$-gravity is given by the Weyl action

$$
S=\int d^{4} x \sqrt{-g} \frac{1}{g_{\text {grav }}^{2}} C_{\mu \nu \rho \sigma} C^{\mu \nu \rho \sigma},
$$

where $C_{\mu \nu \rho \sigma}$ is the Weyl tensor and $g_{\text {grav }}$ is the corresponding coupling. This theory resembles Yang-Mills theories in many respects. It is classically conformally invariant and multiplicatively renormalizable at one loop. Concerning unitarity the same remarks apply as for the full $R^{2}$ theory.

Thus higher derivative gravity actions do not seem to make sense as starting points for the definition of fundamental theories. They play, however, an important role as low energy effective actions of fundamental quantum theories of gravity such as string theory. In string theory one can show that the low energy effective action indeed contains higher curvature terms [5, 7]. The couplings of the effective theory are functions of the scalar fields. One finds that $R^{2}$-terms are generated both through loop corrections and through stringy $\alpha^{\prime}$-corrections. The parameter $\alpha^{\prime}$, which has dimension length squared (in natural units) is the fundamental dimensionful parameter of string theory. Up to a constant, $\alpha^{\prime}$ is the inverse of the string tension. One then defines the string length by $l_{\text {String }}=\left(\alpha^{\prime}\right)^{1 / 2}$ and the string mass $m_{\text {String }}=\left(2 \alpha^{\prime}\right)^{-1 / 2}$. String units are obtained by setting $\alpha^{\prime}=\frac{1}{2}$, so that the string mass equals unity. When computing the low energy effective action of the massless modes of string theory at tree level one finds

\footnotetext{
${ }^{2}$ The definitions of the curvature tensors are recalled in appendix A.
} 
that it contains an Einstein-Hilbert term together with an infinite series of higher curvature terms that are supressed by powers of $\alpha^{\prime}$, so that they are subleading at low energies. Thus, string theory deviates from Einstein gravity already at the classical level. These are the $\alpha^{\prime}-$ corrections mentioned above. On top of this the effective action gets contributions from loop corrections. String theory contains a dimensionful coupling constant $\kappa$, which can be identified with the gravitational coupling, i.e. the coefficient of the Einstein-Hilbert term. This quantity is not independent from the other dimensionful constant, $\alpha^{\prime}$. Besides the graviton, every string theory contains another universal state, a massless scalar called the dilaton $\phi$. The dependence of string perturbation theory on this field is such that the $g$-loop contribution to the effective action is proportional to $\left(e^{-2 \phi}\right)^{1-g}$. Therefore there is a relation between $\kappa, \alpha^{\prime}$ and the vacuum expectation value $\langle\phi\rangle$ of the dilaton, which in four dimensions reads

$$
\frac{\alpha^{\prime}}{4}=\frac{\kappa^{2}}{8 \pi} e^{2\langle\phi\rangle} .
$$

The vacuum expectation value of the dilaton is not fixed by the equations of motion and this degeneracy is not lifted in string perturbation theory.[- Therefore $\langle\phi\rangle$ is a free parameter, at least in perturbation theory, and has to be specified as part of the definition of a string background.

Thus there is one fundamental dimensionful parameter which can be taken to be $\kappa$ or $\alpha^{\prime}$ and a continous familiy of vacua parametrized by $\langle\phi\rangle$. Since $e^{\langle\phi\rangle}$ can serve as loop counting parameter one introduces the dimensionless string coupling

$$
g_{S}=e^{\langle\phi\rangle} .
$$

In terms of the string and Planck masses, the relation (2.5) can be rewritten as

$$
m_{\text {String }}=g_{S} m_{\text {Planck }} .
$$

It should be noted that whether an $R^{2}$-term is due to a loop or due to an $\alpha^{\prime}$-correction is not determined by the term itself, but is a model (or better background) dependent question, which has to be determined case by case. The Einstein-Hilbert term itself is always found at tree level in both $g_{S}$ and $\alpha^{\prime}$. Consider now an $R^{2}$-term, by which we denote any curvature term with four derivatives. In four dimensions where both $\kappa^{2}$ and $\alpha^{\prime}$ have dimension length squared such an $R^{2}$ term can, by dimensional analysis, be of the form $\kappa^{2 m}\left(\alpha^{\prime}\right)^{n} R^{2}$, where $m+n=0$. Thus it could be either a string loop effect $(m=0=n)$ or an $\alpha^{\prime}$-loop effect $(n=1=-m)$. Since the coupling constants of the effective theory are not just constants but field dependent objects the actual analysis is more complicated, as we will see in chapter 5 . There we will consider terms of the form $C^{2} T^{2 g-2}$, where $C$ is the Weyl tensor and $T$ is a field strength. In one specific theory (IIA string theory on a Calabi-Yau threefold) these terms arise at the $g$-loop level in $g_{S}$ whereas in another, dual theory (heterotic string theory on $K 3 \times T^{2}$, with a choice of gauge bundle) the same term gets contributions at tree level, one loop level and non-perturbative level in $g_{S}$. In both cases the terms get loop and non-perturbative contributions in $\alpha^{\prime}$. Moreover in another dual theory (IIB string theory on the mirror Calabi-Yau manifold) the terms are $g$-loop in $g_{S}$ and tree level in $\alpha^{\prime}$.

In the effective action for $R^{2}$-gravity one usually separates the $C^{2}$-term (the square of the Weyl tensor) because it is the only conformally invariant term:

$$
8 \pi \mathcal{L}=-\frac{1}{2} R+\frac{1}{4} \frac{1}{g_{\text {grav }}^{2}} C^{2}+\frac{1}{4} \Theta R_{\mu \nu \rho \sigma}{ }^{\star} R^{\mu \nu \rho \sigma}+\frac{1}{\rho^{2}} R_{\mu \nu} R^{\mu \nu}+\frac{1}{\sigma^{2}} R^{2} .
$$

Here ${ }^{\star} R_{\mu \nu \rho \sigma}=\frac{1}{2} \epsilon_{\mu \nu \alpha \beta} R_{\rho \sigma}^{\alpha \beta}$ is the Hodge dual of the Riemann tensor. The conventional choice of couplings for compactifications of the heterotic string is such that at tree level all higher derivative couplings are topological,

$$
8 \pi \mathcal{L}=-\frac{1}{2} R+\frac{1}{4} \operatorname{Re} S G B+\frac{1}{4} \operatorname{Im} S R_{\mu \nu \rho \sigma}{ }^{\star} R^{\mu \nu \rho \sigma},
$$

\footnotetext{
${ }^{3}$ We only consider supersymmetric string vacua in this paper. If string theory describes our world then supersymmetry must be broken, and the supersymmetry breaking mechanism presumably generates a potential for all scalar fields and in particular for the dilaton.
} 
where $S=e^{-2 \phi}+i a$ is the complex heterotic dilaton, $G B$ is the Gauss-Bonnet term, $R_{\mu \nu \rho \sigma}{ }^{\star} R^{\mu \nu \rho \sigma}$ is proportional to the Hirzebruch signature density and $\operatorname{Im} S$ is sometimes called the 'gravitational $\Theta$-angle' [30, 31, 32].

At the loop level one can in particular study the $C^{2}$-coupling $g_{\text {grav }}$ which is very similar to a gauge coupling (see for example [33] and references therein). We will later study this and related couplings in the context of compactifications with $\mathcal{N}=2$ supersymmetry.

Finally note that the precise relation of quantum corrected string couplings to their counterparts in the effective field theory involves questions such as the scheme dependence of couplings and the proper definition of the renormalization scale [34]. We will not need to discuss this systematically (in the case of gauge couplings this is different because one wants to address the problem of coupling unification) but the distinction between 'physical' and 'Wilsonian' couplings [35] will play some role later in chapters 5 and 6 .

\subsection{Black Holes}

\subsubsection{Basic Properties of Black Holes}

Intuitively a black hole is a region of space-time from which one cannot escape. A more precise definition is that a black hole is an asymptotically flat space-time containing a region which is not in the backward lightcone of future timelike infinity [21, 22]. The boundary of such a region is a null hypersurface, called the future event horizon, or event horizon for short. The event horizon is a surface of infinite redshift and this motivates the name black hole.

Already one of the most simple solutions of Einstein gravity, the Schwarzschild solution, describes a black hole. The Schwarzschild solution is the unique spherically symmetric, and therefore, by Birkhoffs theorem, static solution of the vacuum Einstein equations. It describes the exterior region of a spherical mass (or better energy-momentum) distribution. If the massive body is sufficiently compact, the solution exhibits an event horizon. Since no mechanism is known which can stop the collapse of a star with a mass above the Oppenheimer-Volkov limit, this is believed to be a physical solution which describes the classical final state of a spherical collapse.

There exist more general black hole solutions which in addition carry charge and angular momentum. In Einstein-Maxwell theory one can show that the most general stationary black hole is the Kerr-Newmann black hole, which is uniquely characterized by its mass, charge and angular momentum. Remember that a space-time is called stationary if it is time-independent, i.e. if it posesses a timelike Killing vector. Such space-times represent classical final states of a total gravitational collapse. Much of the work on black holes focusses on stationary black holes, because these solutions are analytically tractable. In the following we will restrict ourselves to the subclass of static black holes, with the exception of some remarks in this introductory chapter. A stationary space-time is called static, if the timelike Killing vector field is hypersurface orthogonal. $\mathrm{f}$ A metric which is both static and spherically symmetry can be brought to the form

$$
d s^{2}=-e^{2 h(r)} d t^{2}+e^{2 k(r)} d r^{2}+r^{2} d \Omega^{2}
$$

by a coordinate transformation. Here $d \Omega^{2}=\sin ^{2} \theta d \phi^{2}+d \theta^{2}$ denotes the standard metric on the unit sphere. The adapted coordinates $t, r, \phi, \theta$ provide a generalization of spheric coordinates in Minkowski space-time.

The most general static black hole of Einstein-Maxwell theory is given by the ReissnerNordstrøm solution, which has a metric of the form (2.10), with

$$
e^{2 h(r)}=e^{-2 k(r)}=1-\frac{2 M}{r}+\frac{Q^{2}}{r^{2}},
$$

\footnotetext{
${ }^{4}$ We will not consider other types of event horizons. The technical terms used in this section will be explained in some more detail in the following sections. A complete account of background material is provided by textbooks on general relativity, see in particular [21].

${ }^{5}$ This notion is defined in section 2.2.4.
} 
where $M, Q$ are the mass and charge carried by the black hole, respectively. The mass of an asymptotically flat space time can be determined by considering the non-relativistic motion of a test particle in the asymptotic region. Such a particle sees a Newtonian gravitational potential $V=-M / r$, where $M$ is related to the $1 / r$ deviation of the metric from flat space by $g_{00}=-\left(1-\frac{2 M}{r}+\cdots\right)$. A more elaborate definition of the mass is provided by the ADM mass, see for example [21]. Like the mass, the charge can be measured at infinity, and is defined by

$$
Q=\frac{1}{4 \pi} \oint_{S_{\infty}^{2}}{ }^{\star} F
$$

where $S_{\infty}^{2}$ is an asymptotic spacelike sphere at infinity and ${ }^{\star} F$ is the dual of the field strength two-form $F=\frac{1}{2} F_{\mu \nu} d x^{\mu} \wedge d x^{\nu}$. World indices corresponding to the adapted coordinates are denoted by $\mu, \nu=t, r, \phi, \theta$. The normalization is such that an electric field carries charge $Q$ if its asymptotic form at infinity is

$$
F_{t r}=\frac{Q}{r^{2}} .
$$

We have restricted ourselves here to electric charge, but it is straigthforward to include magnetic charge,

$$
P=\frac{1}{4 \pi} \oint_{S_{\infty}^{2}} F
$$

as well. This results in replacing $Q^{2}$ by $Q^{2}+P^{2}$ in the metric.

Besides the mass and the charge, which are quantities measured at infinity, there are two characteristic quantities defined on the event horizon. One is the surface gravity $\kappa_{S}$, which measures the strength of the gravitational field on the event horizon, the other is the area of the event horizon. These quantities have a tentative thermodynamic interpretation, and we will discuss them in more detail soon.

The interpretation of the Reissner-Nordstrøm solution depends on the values of mass and charge. If $M>|Q|$ (if magnetic charge is present one has to replace $|Q|$ by $\sqrt{Q^{2}+P^{2}}$ ) then the solution describes a black hole with two horizons, an exterior event horizon and an interior so-called Cauchy horizon. . $^{0}$ This is the non-extremal Reissner-Nordstrøm black hole. Its surface gravity is given by

$$
\kappa_{S}=\frac{\sqrt{M^{2}-Q^{2}}}{2 M\left(M+\sqrt{M^{2}-Q^{2}}\right)-Q^{2}},
$$

whereas the area of the event horizon is

$$
A=4 \pi\left(M+\sqrt{M^{2}-Q^{2}}\right)^{2} .
$$

If $M=|Q|$, the two horizons coincide and the surface gravity vanishes. The area is now given in terms of the charge by

$$
A=4 \pi Q^{2} .
$$

The resulting black hole is called the extremal Reissner-Nordstrøm black hole. If $M<|Q|$ the event horizon vanishes and one is left with a naked singularity, which according to the cosmic censorship hypothesis is considered to be an unphysical solution. Black hole solutions must respect the mass bound $M \geq|Q|$ to ensure the existence of an event horizon. Extremal black holes saturate the bound.

Finally note that the special case $M>0, Q=0$ is the Schwarzschild black hole, while $M=Q=0$ is flat Minkowski space.

\subsubsection{The Laws of Black Hole Mechanics}

One of the most remarkable results of black hole physics is that one can derive a set of laws, called the laws of black hole mechanics, which have the same structure as the laws of thermodynamics 36. The black hole laws are a priori not linked to thermodynamics in any obvious way,

\footnotetext{
${ }^{6}$ See [21, 22] for a detailed account.
} 
because they are derived using geometrical properties of event horizons and general covariance. We will first describe the black hole laws in the context of Einstein gravity coupled to matter. Later we will discuss their generalization to higher derivative gravity.

The zeroth law states that the surface gravity of a stationary black hole is constant over the event horizon,

$$
\kappa_{S}=\text { const. }
$$

This resembles the zeroth law of thermodynamics, which says that the temperature is constant in thermodynamic equilibrium. The first law of black hole mechanics is energy conservation. One considers two infinitesimally close stationary black hole solutions and expresses the change $\delta M$ of the mass in terms of changes of the area of the event horizon, $\delta A$, of the charge, $\delta Q$, and of the angular momentum, $\delta J$ :

$$
\delta M=\frac{\kappa_{S}}{8 \pi} \delta A+\mu \delta Q+\Omega \delta J .
$$

This has the same form as the first law of thermodynamics, and since $\kappa_{S}$ is the analogue of temperature the area plays the role of entropy. This is the fact we are most interested in. The parameter associated with a variation of the charge is $\mu=Q / r_{+}$, where $r_{+}$is the location of the event horizon 7 and $\Omega$ is the angular velocity of rotation of the black hole. Formally we have the first theorem of thermodynamics for a grand canonical ensemble with $\Omega$ and $J$ playing the roles of pressure and volume and $\mu$ and $Q$ playing the roles of chemical potential and particle number, respectively. This form of the first law applies to Kerr-Newmann solutions of Einstein-Maxwell theory. If a more complicated matter sector is present, then there are more terms [37].

The analogy of area and entropy is confirmed by the second law of black hole mechanics, the Hawking area law [38]. This is a statement about non-stationary processes in a space-time containing black holes, including collisions and fusions of black holes.] Two assumptions have to be made: 1.) The time evolution of the system must be under sufficient control. This is implemented by requiring that the space-time is 'strongly asymptotically predictable'. 2.) The matter, represented by the stress energy tensor must behave 'reasonable'. This is done by imposing the null energy condition on the stress energy tensor. We refer to [21] for a more detailed explanation. Under these assumptions the second law states that the total area of all event horizons is non-decreasing,

$$
\delta A \geq 0 .
$$

This is a striking analogue of the entropy law of thermodynamics.

Finally there is a third law of black hole mechanics. Here several versions exist, and the status of this law does not seem to be fully understood. We only touch upon this and refer to 21 for a more detailed account. One version of the law states that the extremal limit cannot be reached in finite time in any 'physical process'. Here the obvious problem is to define what a physical process is and to bring such non-stationary processes under sufficient control. Another version, which does not refer to non-stationary properties, states that black holes of vanishing 'temperature' (surface gravity) have vanishing entropy. This is in obvious contradiction to the fact that the area of an extremal black hole can be non-vanishing. There are however subtleties at the quantum level, and these have been used as arguments in favour of the second version of the third law. We will return to this when discussing quantum aspects of black holes.

\subsubsection{Quantum Aspects of Black Holes and Black Hole Thermody- namics}

The laws of black hole mechanics have been known for quite some time, but were mostly considered as a curious formal analogy. The most obvious reason for not believing in a thermodynamic

${ }^{7}$ For the Reissner-Nordstrøm black hole the outer horizon is $r_{+}=M+\sqrt{M^{2}-Q^{2}}$ in the coordinates used in $(2.10)$.

${ }^{8}$ A black hole cannot split into two or more black holes, see for example 21. Note that the notion of a black hole is not time reflection symmetric even if the black hole is static, because it is based on the concept of a future event horizon. One can define analogue space-times involving past even horizons, which sometimes are called white holes. 
content is that a classical black hole is just black: It cannot radiate and therefore one should assign temperature zero to it, so that the interpretation of the surface gravity as temperature has no physical content.

This changes dramatically when taking into account quantum effects. One can analyse black holes in the context of quantum field theory in curved backgrounds, where matter is described by quantum field theory while gravity enters as a classical background, see for example [39]. In this framework it was discoverd that black holes can emit Hawking radiation [40]. The spectrum is (almost (9) $^{9}$ Planckian with a temperature, the so-called Hawking temperature, which is indeed proportional to the surface gravity,

$$
T_{H}=\frac{\kappa_{S}}{2 \pi} .
$$

This motivates to take the analogy of area and entropy seriously. Since the Hawking temperature fixes the factor of proportionality between temperature and surface gravity, one finds the Bekenstein-Hawking area law,

$$
\mathcal{S}=\frac{A}{4} .
$$

Before the discovery of Hawking radiation, Bekenstein had already given an independent argument in favour of assigning entropy on black holes 42, 43]. He pointed out that in a space-time containing a black hole one could adiabatically transport matter into it. This reduces the entropy in the observable world and thus violates the second law of thermodynamics. He therefore proposed to assign entropy to black holes, such that a generalized second law is valid, which states that the sum of thermodynamic entropy and black hole entropy is non-decreasing. With the discovery of Hawking radiation one can give an additional argument in favour of this generalization: By Hawking radiation a black hole looses mass and shrinks. This is not in contradiction with the second law of black hole mechanics, because one can show that the null energy condition is violated in the near horizon region if the effect of quantum fields is taken into account. Bekenstein's generalized second law claims that the loss in black hole entropy is always (at least) compensated by the thermodynamic entropy of the Hawking radiation, so that the total entropy is non-decreasing.

One example of unusual thermodynamic behaviour of black holes is provided by the mass dependence of the temperature of uncharged black holes. For the Schwarzschild black hole one finds $\kappa_{S}=(4 M)^{-1}$, which shows that the specific heat is negative: The black hole heats up while loosing mass. This behaviour is unusual, but nevertheless not unexpected because gravity is a purely attractive force. The fact that uncharged black holes seem to fully decay into Hawking radiation leads to the information or unitarity problem of quantum gravity, see for example [11]. Charged black holes behave differently in that the Hawking temperature vanishes in the extremal limit. Therefore extremal black holes are stable against decay by thermic radiation. It is less clear whether they are absolutely stable in non-supersymmetric gravity, since they could decay through charge superradiance [1]. But in (extended) supergravity one can argue that they are absolutely stable and provide examples of solitons, as we will see later.

We already mentioned that one version of the third law states that extremal black holes have vanishing entropy. This statement depends on subtleties of the quantum mechanical treatment of such objects [44, 35]: The entropy can be computed in semiclassical quantum gravity, i.e. by quantizing gravity around a black hole configuration. One can either use the Euclidean path integral formulation or the Minkowskian canonical framework. The result for the entropy depends on whether the extremal limit is taken before or after quantization: If one quantizes around extremal black holes the entropy vanishes. But if one quantizes around general charged black hole configurations one finds an entropy that is non-vanishing when taking the extremal limit. The second option seems to be more natural and it is the one supported by string theory, as we will see later.

The identification of the area with entropy leads to several questions. Standard thermodynamics provides a macroscopic effective description of systems in terms of coarse grained

\footnotetext{
9 When backscattering is taken into account the spectrum is not really Planckian. Nevertheless it makes sense to assign a temperature to a black hole, because a black hole can bein thermic equilibrium with a thermal bath if one puts it into a finite box with perfectly reflecting walls [39, 1, 41].
} 
macroscopic observables like temperature and entropy. At the fundamental, microscopic level systems are described by statistical mechanics in terms of microstates which encode, say, the positions and momenta of all particles that constitute the system. At the microscopic level one can define the microscopic or statistical entropy as the quantity which characterizes the degenaracy of microstates in a given macrostate, where the macrostate is characterized by specifying the macroscopic observables. Assuming ergodic behaviour the macroscopic and microscopic entropy agree.10 One should therefore address the question whether there exists a fundamental, microscopic level of description of black holes, where one can identify microstates and count how many of them lead to the same macrostate. The macrostate of a black hole is characterized by its mass, charge and angular momentum. Denoting the number of microstates leading to the same mass $M$, charge $Q$ and angular momentum $J$ by $N(M, Q, J)$, the statistical or microscopic black hole entropy is defined by

$$
\mathcal{S}_{\text {micro }}=\log N(M, Q, J) .
$$

If the Bekenstein-Hawking entropy is the analogue of thermodynamic entropy and if stationary black holes are the analogue of thermodynamic equilibrium states, then the Bekenstein-Hawking entropy must coincide with the microscopic entropy,

$$
\mathcal{S}=\mathcal{S}_{\text {micro }}
$$

We will see that the microscopic picture that string theory gives us for extremal black holes leads to quantitative agreement between the two entropies.

One of the astonishing properties of the Bekenstein-Hawking entropy is its simple and universal behaviour: the entropy is just proportional to the area. The fact that the entropy is proportional to the area and not to the volume has led to the speculation that quantum gravity is in some sense non-local and admits a holographic representation on boundaries of space-time 45. 46]. The D-brane picture of black holes [12, 47, 48, 49, 50, 51] (see [13] for a review) and the AdS/CFT conjecture [52, 53, 54] (see [55 for a review) provide steps towards a concrete realization of this proposal. The amount of information that one can store in a black hole according to the Bekenstein-Hawking area law is roughly one bit per Planck length squared. This suggests a picture where gravity lives on a two-dimensional lattice of Planck length spacing. It is also remarkable that the Bekenstein-Hawking entropy is several orders of magnitude bigger than the thermodynamic entropy of a star of the same mass [1]. Every microscopic picture of black holes has to explain where this huge number of microstates comes from. In string theory four-dimensional extremal black holes are effective descriptions of complicated bound states of solitons of the underlying ten- or eleven-dimensional fundamental theory. These configurations have a huge number of internal excitations which all lead to the same four-dimensional black hole. This accounts for the huge entropy.

\subsubsection{Black Hole Horizons}

In the next sections we will discuss the generalization of the laws of black hole mechanics to higher derivative gravity. At the same time we will explain the definitions of surface gravity and macroscopic black hole entropy in some more detail. We start in this section by reviewing properties of black hole horizons that apply to both Einstein gravity and higher derivative gravity. Then we formulate the generalization of the zeroth law to higher derivative gravity.

Our discussion of event horizons follows [21]. The event horizon is a hypersurface in spacetime. A space-time hypersurface $\Delta$ can be defined by an equation $f(x)=0$. Alternatively one can specify the hypersurface in terms of its normal vector field $n^{\mu}(x)$, where $n^{\mu}(x) t_{\mu}(x)=0$ for all tangent vectors $t^{\mu}(x)$ of $\Delta$. If $\Delta$ is defined in terms of a function $f(x)$, then $\nabla_{\mu} f$ is

\footnotetext{
${ }^{10}$ It is somewhat difficult to imagine how gravity, which is purely attractive, can show in some sense ergodic behaviour [41]. This is one of the problems in relating thermodynamics and gravity. We will see that nevertheless one can define and compute the microscopic entropy for certain black holes in string theory and that it quantitatively agrees with the macroscopic black hole entropy, as defined by the Bekenstein-Hawking area law and generalizations thereof.
} 
automatically normal to $\Delta$. According to (a special case of) the Frobenius theorem a vector field $n^{\mu}$ is the normal vector field of a smooth family of submanifolds if and only if it is hypersurface orthogonal,

$$
n_{[\mu} \nabla_{\nu} n_{\rho]}=0 .
$$

A hypersurface is called a null surface if its normal vector field is null, $n^{\mu} n_{\mu}=0$. Such surfaces appear as boundaries of lightcones. Event horizons are per definition null hypersurfaces. Note that for a null hypersurface a normal vector is at the same time a tangent vector.

By taking a spacelike cross section $\Sigma$ of $\Delta$ one obtains a two-dimensional spacelike surface which is also called the horizon. We will distinguish both objects by use of the corresponding symbols. Later on we will make use of the so-called normal bivector or binormal $\varepsilon_{\mu \nu}$ of $\Sigma$. In the normal space of $\Sigma$ in space-time one can construct one linear independent antisymmetric tensor. By imposing the normalization $\varepsilon_{\mu \nu} \varepsilon^{\mu \nu}=-2$ one can make a unique choice (up to an overall sign which is fixed by choosing an orientation). This tensor is the binormal of $\Sigma$. Since the normal space to $\Sigma$ has signature (-+) one can write $\varepsilon_{\mu \nu}$ as the exterior product of two null vectors. One can take one of these to be the normal vector $n_{\mu}$ and chooses a second linear independent null vector $N^{\mu}$, normalized such that $N^{\mu} n_{\mu}=-1$. Then the bivector

$$
\varepsilon_{\mu \nu}=N_{\mu} n_{\nu}-N_{\nu} n_{\mu}
$$

has its non-vanishing components in the directions normal to $\Sigma$ and has the required normalization.

If the black hole is static and spherically symmetric, then one can bring the metric to the form

$$
d s^{2}=-e^{2 g(r)} d t^{2}+e^{2 f(r)}\left(d r^{2}+r^{2} d \Omega^{2}\right),
$$

where $(t, r, \phi, \theta)$ are isotropic coordinates $\square$ For this metric the binormal takes the form

$$
\varepsilon_{t r}=-\varepsilon_{r t}=e^{g(r)+f(r)} .
$$

A Killing horizon is defined to be a null hypersurface which has a Killing vector field as normal vector field. Note that this notion is independent from that of an event horizon. In Einstein gravity all event horizons of stationary black holes are Killing horizons [22]. In higher derivative gravity one can show that event horizons are Killing horizons if the black hole is static or if it is stationary, axisymmetric and possesses a discrete reflection symmetry, called $t-\phi$ reflection symmetry 12 [56, 57, 58]. In the following it is understood that event horizons are Killing horizons and in particular that stationary black holes in higher derivative gravity are required to be in addition axisymmetric and to have $t-\phi$ reflection symmetry.

Remember that Killing vectors are defined by the Killing equation

$$
\nabla_{(\mu} \xi_{\nu)}=0
$$

This has the consequence that the second derivative of a Killing vector is given by

$$
\nabla_{\mu} \nabla_{\nu} \xi_{\rho}=R_{\nu \rho \mu}^{\sigma} \xi_{\sigma}
$$

and therefore all higher derivatives of a Killing vector can be expressed in terms of the Killing vector and its curl $\nabla_{[\mu} \xi_{\nu]}=\nabla_{\mu} \xi_{\nu}$.

For Killing horizons one can define the surface gravity $\kappa_{S}$. If $\xi^{\mu}$ is the Killing vector field normal to the horizon, or horizontal Killing vector field for short, then one can define $\Delta$ by $f=\xi^{\mu} \xi_{\mu}=0$. Since $\nabla_{\mu} f$ is normal to $\Delta$, it must be proportional to $\xi^{\mu}$ itself. The coefficient of proportionality defines the surface gravity,

$$
\nabla_{\mu}\left(\xi^{\nu} \xi_{\nu}\right)=-2 \kappa_{S} \xi_{\mu}
$$

\footnotetext{
${ }^{11}$ Isotropic coordinates have the special property that the spatial part of the metric is conformally flat. Note that the above coordinates only cover the exterior region of the black hole. The event horizon is localized at $r=0$. The coordinate system (2.10) used in the last section also covers the interior of the black hole and $r=0$ is the postion of the singularity.

${ }^{12}$ In terms of coordinates this means that mixed $t-\phi$ components of the metric can be transformed away.
} 
Using the Frobenius theorem (2.25) and the Killing equation (2.29) one can derive several useful relations. In particular one can show

$$
\kappa_{S}^{2}=-\frac{1}{2}\left(\nabla^{\mu} \xi^{\nu}\right)\left(\nabla_{\mu} \xi_{\nu}\right) \text { and } \xi^{\mu} \partial_{\mu} \kappa_{S}=0 .
$$

The second equation implies that the surface gravity is constant along the integral lines of the Killing field $\xi^{\mu}$ on $\Delta$. The zeroth law of black hole mechanics states that the surface gravity is constant over $\Delta$ for static and stationary black holes [36, 57, 58].

For static black holes one can give an alternative definition of the surface gravity [21]. One considers the problem of keeping a test body at rest on the event horizon. Though the local force on the body is infinite (which is another way of defining an event horizon) the force on an external observer who tries to keep the testbody fixed is redshifted and finite. If one fictionally refers to an observer at infinity then the redshifted force per mass equals the surface gravity as defined above in terms of the Killing vector. In this sense the surface gravity measures the force of the gravitational field on the event horizon. Note that this definition does not apply to stationary, rotating black holes, because in this case it is not possible to keep a body at rest inside the ergosphere. Nevertheless the surface gravity, as defined by the Killing vector is an intrinsic property of the horizon and characterizes, in a less direct way, the gravitational field.

\subsubsection{Surface Charges}

In order to define black hole entropy in a way that applies to higher derivative gravity one can make use of a formalism which associates surfaces charges to local symmetries of the Lagrangian. If the surface charge is evaluated in an on-shell background with a rigid symmetry then one gets a Noether charge in the usual sense, i.e. the generator of the rigid symmetry of the background. The black hole entropy is an example of such a Noether charge, and the residual gauge symmetry is the isometry generated by the horizontal Killing vector field.

The method of Noether surface charges was proposed by R. Wald in [18], based on [59, 60] and further developed by him and other authors in 61, 62, 63. In this section we will review a modified algorithm for computing surface Noether charges that was described in [64, 65].

We will consider a generally covariant Lagrangian that depends on the Riemann tensor but does not contain derivatives of the Riemann tensor. ${ }^{13}$ In addition the Lagrangian depends on a matter field $\psi_{\mu \nu}$ (which is a second rank tensor with no particular symmetry) and on its first derivative. The Lagrangian is not strictly invariant under general coordinate transformations, but transforms into a total derivative. Consider now a variation of the fields $\phi=\left(g_{\mu \nu}, \psi_{\mu \nu}\right)$ that takes the form of a general coordinate transformation multiplied with a test function $\epsilon(x)$ :

$$
\begin{aligned}
\delta_{\xi} g_{\mu \nu} & =-\epsilon(x)\left(\nabla_{\mu} \xi_{\nu}(x)+\nabla_{\nu} \xi_{\mu}(x)\right), \\
\delta_{\xi} \psi_{\mu \nu} & =-\epsilon(x)\left(\nabla_{\mu} \xi^{\sigma} \psi_{\sigma \nu}+\nabla_{\nu} \xi^{\sigma} \psi_{\mu \sigma}+\xi^{\sigma} \nabla_{\sigma} \psi_{\mu \nu}\right) .
\end{aligned}
$$

Such a transformation is not a symmetry of the Lagrangian, but if $\epsilon(x)$ and its derivatives satisfy the boundary conditions required by Hamiltons variational principle, then the variation is proportional to the equations of motion. Therefore the variation is a total derivative $\partial_{\mu} J^{\mu}$, modulo the equations of motion. This provides the definition of the Noether current associated with the local transformation generated by $\xi_{\mu}(x) .14$ Explicitly one finds

$$
\begin{aligned}
J^{\mu}= & \xi^{\mu} \mathcal{L}-2 \mathcal{L}^{\mu \nu \rho \sigma}\left[R_{\lambda \nu \rho \sigma} \xi^{\lambda}+\nabla_{\nu} \nabla_{\rho} \xi_{\sigma}\right]+4 \nabla_{\rho} \mathcal{L}^{\mu \nu \rho \sigma} \nabla_{(\nu} \xi_{\sigma)} \\
& -\mathcal{L}_{\psi}^{\mu, \rho \sigma}\left[\nabla_{\rho} \xi^{\lambda} \psi_{\lambda \sigma}+\nabla_{\sigma} \xi^{\lambda} \psi_{\rho \lambda}+\xi^{\lambda} \nabla_{\lambda} \psi_{\rho \sigma}\right]
\end{aligned}
$$

\footnotetext{
${ }^{13}$ The algorithm of 62 can be used when the Lagrangian depends on covariant derivatives of the Riemann tensor, whereas our algorithm has so far only been developed for a restricted class of Lagrangians, which contains the $\mathcal{N}=2$ supergravity Lagrangians that will be studied later.

14 As discussed in 64] this definition differs from the algorithm of 18, 61, 62, by improvement terms. This reflects ambiguities in the construction of the Noether current which, however, do not affect the definition of entropy as a Noether charge.
} 


$$
\begin{aligned}
+\frac{1}{2}\left(\nabla_{\lambda} \xi_{\rho}+\nabla_{\rho} \xi_{\lambda}\right)\left[\mathcal{L}_{\psi}^{\mu, \rho \sigma} \psi^{\lambda}{ }_{\sigma}+\mathcal{L}_{\psi}^{\mu, \sigma \rho} \psi_{\sigma}{ }^{\lambda}+\mathcal{L}_{\psi}^{\rho, \mu \sigma} \psi^{\lambda}{ }_{\sigma}\right. \\
\left.+\mathcal{L}_{\psi}^{\rho, \sigma \mu} \psi_{\sigma}{ }^{\lambda}-\mathcal{L}_{\psi}^{\rho, \lambda \sigma} \psi^{\mu}{ }_{\sigma}-\mathcal{L}_{\psi}^{\rho, \sigma \lambda} \psi_{\sigma}{ }^{\mu}\right],
\end{aligned}
$$

where

$$
\mathcal{L}^{\mu \nu}=\frac{\partial \mathcal{L}}{\partial g_{\mu \nu}}, \quad \mathcal{L}^{\mu \nu \rho \sigma}=\frac{\partial \mathcal{L}}{\partial R_{\mu \nu \rho \sigma}}, \quad \mathcal{L}_{\psi}^{\rho, \mu \nu}=\frac{\partial \mathcal{L}}{\partial \nabla_{\rho} \psi_{\mu \nu}}
$$

are partial derivatives of the Lagrangian ${ }^{15}$ with respect to the tensors $g_{\mu \nu}, R_{\mu \nu \rho \sigma}$ and $\nabla_{\rho} \psi_{\mu \nu}$. Since these tensors have symmetries their components are not independent and the definition of a partial derivative is ambiguous. To fix this we impose that the derivative has the same symmetry properties as the tensor, i.e. the partial derivative is determined by the variation of the Lagrangian as a function of the tensor field,

$$
\delta \mathcal{L}=\frac{\partial \mathcal{L}}{\partial T_{\mu_{1} \cdots \mu_{n}}} \delta T_{\mu_{1} \cdots \mu_{n}}+\cdots
$$

The Noether current is a local function of the fields $\phi$ and depends on the transformation parameter $\xi_{\mu}(x)$ only through the variations $\delta_{\xi} \phi$.

One can directly verify, using the equations of motion, that the Noether current is conserved on-shell. Moreover one can use the equations of motion to write the current in terms of a Noether protential $Q^{\mu \nu}=Q^{[\mu \nu]}$ as $J^{\mu}=\nabla_{\nu} Q^{\mu \nu}$, so that current conservation becomes trivial. The explicit expression for the Noether potential for the case at hand is

$$
\begin{aligned}
Q^{\mu \nu}= & -2 \mathcal{L}^{\mu \nu \rho \sigma} \nabla_{\rho} \xi_{\sigma}+4 \nabla_{\rho} \mathcal{L}^{\mu \nu \rho \sigma} \xi_{\sigma} \\
+ & +\frac{1}{2}\left[-\mathcal{L}_{\psi}^{\mu, \nu \rho} \psi^{\sigma}{ }_{\rho}-\mathcal{L}_{\psi}^{\mu, \rho \nu} \psi_{\rho}{ }^{\sigma}+\mathcal{L}_{\psi}^{\mu, \sigma \rho} \psi^{\nu}{ }_{\rho}\right. \\
& \left.\quad+\mathcal{L}_{\psi}^{\sigma, \mu \rho} \psi^{\nu}{ }_{\rho}+\mathcal{L}_{\psi}^{\mu, \rho \sigma} \psi_{\rho}{ }^{\nu}+\mathcal{L}_{\psi}^{\sigma, \rho \mu} \psi_{\rho}{ }^{\nu}-(\mu \leftrightarrow \nu)\right] \xi_{\sigma} .
\end{aligned}
$$

The Noether potential is not uniquely defined because one can add terms of the form $\nabla_{\rho} X^{\mu \nu \rho}$, where $X^{\mu \nu \rho}=X^{[\mu \nu \rho]}$. It is a local function of the fields and of the transformation parameter: $Q^{\mu \nu}=Q^{\mu \nu}(\phi, \xi)$.

The surface charge associated to the transformation $\xi_{\mu}(x)$ is defined by integrating the Noether potential over a closed spacelike surface $\Sigma$,

$$
Q=\oint_{\Sigma} Q^{\mu \nu} d \Sigma_{\mu \nu}
$$

The surface charge is a Noether charge in the usual sense if $\xi_{\mu}(x)$ is a Killing vector. In this case the transformation parametrized by $\xi_{\mu}(x)$ is a symmetry of the background. The Noether potential is proportional to a field dependent linear combination $\xi^{\mu}$ and its curl $\nabla_{[\mu} \xi_{\nu]}$, because all higher derivatives of the Killing vector can be expressed through these two terms.

\subsubsection{The First Law in Higher Derivative Gravity}

We now turn to the first law of black hole dynamics in the context of higher derivative gravity. As we will see the requirement that the first law is valid determines the expression for the entropy. In presence of higher curvature terms the validity of the first law implies that one has to deviate from the Bekenstein-Hawking area law. The idea for the derivation of the first law and of the construction of the entropy can be motivated by analysing the content of the first law in Einstein gravity

$$
\delta M=\frac{\kappa_{S}}{2 \pi} \delta \mathcal{S}+\Omega \delta J
$$

\footnotetext{
${ }^{15}$ In this and in the following sections $\mathcal{L}$ is a function, not a density: $S=\int d^{4} x \sqrt{-g} \mathcal{L}$. In the later chapters we follow supergravity conventions and absorbe the density $\sqrt{-g}$ in the Lagrangian.

${ }^{16}$ We neglect improvement terms $\nabla_{\rho} X^{[\mu \nu \rho]}$ in the following, because they do not contribute to the Noether charge.
} 
Note that this is an astonishing statement because it relates variations of the quantities defined at infinity, the mass $M$ and angular momentum $J$, to quantities defined on the horizon, $\kappa_{S}$ and $\mathcal{S}$, without making reference to the behaviour of the field configuration in the bulk of space time. This suggests that the first law relates the variations of surface charges associated with infinity to variations at the event horizon.

In the above version of the first law we omitted the contributions from electric and magnetic charges and from scalar fields. The presence of matter fields enters into the first theorem in two ways. First there is an explicit contribution to the mass. Since we are interested in the structure of the entropy term, we do not need to care about this. Second the presence of matter fields modifies the equations of motion and this relevant for our discussion because the first theorem refers to solutions to the field equations. But in section 2.2.5 we derived the formula for surface charges in on-shell backgrounds, and this formula holds irrespective of the details of the equations of motion. Since we will see that the entropy is such a surface charge, we do not need to consider the possible additional terms in the first law.

We will now review the proof of the first law given in 18 . Let us consider a continous family of static or stationary black hole solutions. The variation connecting two infinitesimally close solutions is denoted by $\delta$. All solutions in the family have the same symmetries and $\delta$ relates the corresponding Killing vectors and Noether currents. The natural surface charge in a black hole space-time is the one associated with the horizontal Killing vector field. For static black holes this is just the static Killing vector field of time translation invariance, $\xi=\frac{\partial}{\partial t}$, where as for rotating, stationary black holes it is a linear combination of the static and the axial Killing vector field,

$$
\xi=\frac{\partial}{\partial t}+\Omega \frac{\partial}{\partial \phi} .
$$

In the last section we discussed the construction of surface charges both for general transformations and for the special case of Killing vectors. The corresponding variation of the geometry was denoted $\delta_{\xi} \cdot[7$

The central observation for deriving the first law is that the motion along the integral lines of the horizontal Killing vector field is generated by a Hamiltonian $H$, which has the property that its variation along the continuous family of solution vanishes, $\delta H=0$ [18]. Before displaying the explicit form of $\delta H$ we need a few definitions. First we introduce a spacelike Cauchy surface $C$, which has two boundary components. One is an asymptotic two-sphere at infinity, $S_{\infty}^{2}$, the other is a spacelike cross-section $\Sigma$ of the horizon hypersurface $\Delta$. Second we define a vector field $\theta^{\mu}$ by considering the variation of the action under $\delta$ which takes the form

$$
\delta S=\int d^{4} x \partial_{\mu}\left(\sqrt{-g} \theta^{\mu}\right)
$$

after imposing the equations of motion. The variation of the Hamiltonian is [18]

$$
\delta H=\delta\left(\int_{C} d \Omega_{\mu} J^{\mu}\right)-\int_{C} d \Omega_{\mu} \nabla_{\nu}\left(\xi^{\mu} \theta^{\nu}-\xi^{\nu} \theta^{\mu}\right),
$$

where $d \Omega_{\mu}$ is the volume element on $C$ and $J^{\mu}$ is the Nother current associated with the horizontal Killing vector field $\xi^{\mu}$. Using the equations of motions one can rewrite the Noether current in terms of the Noether potential and use Stokes theorem,

$$
\delta H=\int_{\partial C} d \Sigma_{\mu \nu}\left(\delta Q^{\mu \nu}-\xi^{\mu} \theta^{\nu}+\xi^{\nu} \theta^{\mu}\right) .
$$

Next one uses $\delta H=0$ and separates the contributions from the two boundary components to get a relation between the surface charges at infinity and at the horizon 18

$$
\int_{\Sigma} d \Sigma_{\mu \nu}\left(\delta Q^{\mu \nu}-\xi^{\mu} \theta^{\nu}+\xi^{\nu} \theta^{\mu}\right)=\int_{S_{\infty}^{2}} d \Sigma_{\mu \nu}\left(\delta Q^{\mu \nu}-\xi^{\mu} \theta^{\nu}+\xi^{\nu} \theta^{\mu}\right) .
$$

\footnotetext{
${ }^{17}$ This should not be confused with the deformation $\delta$ which relates a family of solutions.

${ }^{18}$ The normal is taken with inward direction relative to $C$ for $\Sigma$ and with outward direction for $S_{\infty}^{2}$.
} 
This is a relation which has the structure of the first law and it remains to find the physical interpretation of the terms involved. We start with the terms at infinity. When decomposing the horizontal Killing vector field into the static part $\xi_{(t)}^{\mu}$ and the axial part $\xi_{(\phi)}^{\mu}$ then the variation at infinity takes the form $\delta M-\Omega \delta J$, where

$$
\begin{aligned}
\delta M & =\int_{S_{\infty}^{2}} d \Sigma_{\mu \nu}\left(\delta Q\left[\xi_{(t)}\right]^{\mu \nu}-\xi_{(t)}^{\mu} \theta^{\nu}+\xi_{(t)}^{\nu} \theta^{\mu}\right), \\
\delta J & =\int_{S_{\infty}^{2}} d \Sigma_{\mu \nu}\left(\delta Q\left[\xi_{(\phi)}\right]^{\mu \nu}\right)
\end{aligned}
$$

and $Q\left[\xi_{(t)}\right], Q\left[\xi_{(\phi)}\right]$ are the Noether charges associated with the static and axial Killing vector. Note that there is no contribution proportional to $\xi_{(\phi)}^{[\mu} \theta^{\nu]}$ in the second line because we have taken the intergration surface tangential to the axial Killing vector field. The above expressions suggest to interpret $\delta M$ and $\delta J$ as the variations of the mass and of the angular momentum of the black hole. A priori it is not clear how to define these quantities in higher derivative gravity. If one can find a vector field $b_{\mu}$ such that

$$
\delta \int_{S_{\infty}^{2}} \xi_{(t)}^{\mu} b^{\nu}-\xi_{(t)}^{\nu} b^{\mu}=\int_{S_{\infty}^{2}} \xi_{(t)}^{\mu} \theta^{\nu}-\xi_{(t)}^{\nu} \theta^{\mu}
$$

then one can use

$$
\begin{aligned}
M & =\int_{S_{\infty}^{2}} d \Sigma_{\mu \nu}\left(Q\left[\xi_{(t)}\right]^{\mu \nu}-\xi_{(t)}^{\mu} b^{\nu}+\xi_{(t)}^{\nu} b^{\mu}\right) \\
J & =\int_{S_{\infty}^{2}} d \Sigma_{\mu \nu} Q\left[\xi_{(\phi)}\right]^{\mu \nu}
\end{aligned}
$$

as definitions of mass and angular momentum. In the case of Einstein gravity these expressions correspond to the Komar expressions for mass and angular momentum [18]. The Komar formulae of general relativity apply for mass and angular momentum in space-times with Killing vectors and coincide with the more general ADM expressions which do only assume asymptotic symmetries, namely asymptotic flatness [21]. It is therefore natural to take (2.47) as the definitions of mass and angular momentum in higher derivative gravity.

Next we turn our attention to the variation of the charge on the horizon. If this is the entropy term we are looking at, it must take the form $\frac{\kappa_{S}}{2 \pi} \delta \mathcal{S}$. Obviously one should consider a non-singular, i.e. non-extremal situation, where the surface gravity $\kappa_{S}$ is finite. This will define the entropy of non-extremal black holes and in the spirit of the discussion given in section 2.2.3 the entropy of extremal black holes is defined by taking the extremal limit of the resulting expression.

Now we analyse the Noether potential on the horizon $\Sigma$. Since the Noether potential depends linearly on $\xi^{\mu}$ and its derivatives, it takes the general form

$$
Q^{\mu \nu}=B^{[\mu} \xi^{\nu]}+C \nabla^{[\mu} \xi^{\nu]}
$$

where $B^{\mu}$ and $C$ are local functions of the fields in the Lagrangian. For the special case of a Lagrangian depending on the Riemann tensor but not on its derivatives we found the explicit expression in section 2.2.5. For Lagrangians which also depend on covariant derivatives of the Riemann tensor the explicit form of $Q^{\mu \nu}$ can be found in [62]. Next we use the Frobenius theorem to decompose the curl of the Killing vector as

$$
\nabla_{[\mu} \xi_{\nu]}=\kappa_{S} \varepsilon_{\mu \nu}+t_{[\mu} \xi_{\nu]}
$$

where $\varepsilon_{\mu \nu}$ is the binormal and $t^{\mu}$ is a tangent vector of $\Sigma$. Note that by the Frobenius theorem the non-vanishing components of the tensor $\nabla_{[\mu} \xi_{\nu]}$ must have a non-tangential part. The 
normalization of the purely normal piece proportional to the binormal is fixed by the definition of surface gravity.

The analysis is simplified by a theorem of [58] that states that the horizon $\Delta$ of a nonextremal black hole can be analytically continued such that it becomes a bifurcate horizon. Bifurcate horizons contain a bifurcation surface $\Sigma_{0}$. This is a spacelike surface on which the horizontal Killing vector field vanishes. Examples of bifurcate horizons are provided by the maximally extended Schwarzschild and non-extremal Reissner-Nordstrøm solutions of EinsteinMaxwell gravity. Note that the analytical extension is used as a technical tool. It is not relevant whether the part of space-time containing $\Sigma_{0}$ is physical.

The special properties of the bifurcation surface can now be used to simplify the computation of the black hole entropy. Since $\delta H=0$ is valid for any choice of the Cauchy surface we can take $\Sigma_{0}$ as the interior boundary. The surface charges are by construction conserved under the evolution generated by $H$ and therefore the horizontal surface charge is the same on any spatial section $\Sigma$. We first note that by using $\xi^{\mu}=0$ on $\Sigma_{0}$ we get $\nabla_{\mu} \xi_{\nu}=\kappa_{S} \varepsilon_{\mu \nu}$ on $\Sigma_{0}$. In addition the contribution $\xi^{\mu} \theta^{\nu}-\xi^{\nu} \theta^{\mu}$ to the variation vanishes. Finally define a rescaled Noether potential and charge by

$$
\tilde{Q}^{\mu \nu}=\kappa_{S}^{-1} Q^{\mu \nu} \text { and } \tilde{Q}=\int_{\Sigma_{0}} d \Sigma_{\mu \nu} \tilde{Q}^{\mu \nu}
$$

so that the variation at the horizon takes the form $\frac{\kappa_{S}}{2 \pi} 2 \pi \delta \int_{\Sigma_{0}} \tilde{Q}$. Note that on the bifurcation surface $\Sigma_{0}$ the rescaled Noether charge takes the simplified form $\tilde{Q}^{\mu \nu}=C \varepsilon^{\mu \nu}$.

Collecting all results we obtain the first law,

$$
\frac{\kappa_{S}}{2 \pi} \delta \mathcal{S}=\delta M-\Omega \delta J
$$

with entropy

$$
\mathcal{S}=2 \pi \int_{\Sigma_{0}} d \Sigma_{\mu \nu} \tilde{Q}^{\mu \nu}
$$

Outside the bifurcation surface the rescaled Noether potential is more complicated, because $\xi_{\mu}$ does not vanish. But it has been shown in [61 that the terms in $\tilde{Q}^{\mu \nu}$ that vanish on $\Sigma_{0}$ do not contribute to the entropy when the integral is evaluated on a general spatial cross section $\Sigma$. Thus, the entropy is given by

$$
\mathcal{S}=2 \pi \int_{\Sigma} d \Sigma_{\mu \nu} \tilde{Q}^{\mu \nu}, \quad \tilde{Q}^{\mu \nu}=C \varepsilon^{\mu \nu},
$$

where $\Sigma$ is an arbitrary spatial cross section of $\Delta$. Note that we droped the additional nonvanishing terms in $\widetilde{Q}^{\mu \nu}$, because they do not contribute to the entropy.

If the Lagrangian does not depend on derivatives of the Riemann tensor, then the rescaled Noether potential is given by [61]

$$
\tilde{Q}_{\mu \nu}=-\frac{\partial \mathcal{L}}{\partial R_{\mu \nu \rho \sigma}} \varepsilon^{\rho \sigma},
$$

up to terms proportional to $\xi$. This result also follows using our formula (2.37) and taking into account a conventional normalization factor 2. 9 Thus the entropy is given by

$$
\mathcal{S}=2 \pi \int_{\Sigma} \frac{\partial \mathcal{L}}{\partial R_{\mu \nu \rho \sigma}} \varepsilon^{\mu \nu} \varepsilon^{\rho \sigma} \sqrt{h} d \Omega
$$

It is instructive to check that one indeed gets the Bekenstein-Hawking area law in the case of Einstein gravity. Then the Lagrangian is $8 \pi \mathcal{L}=-\frac{1}{2} R$ and therefore $8 \pi \frac{\partial \mathcal{L}}{\partial R_{\mu \nu \rho \sigma}}=-\frac{1}{2} g^{\mu[\rho} g^{\sigma] \nu}$.

\footnotetext{
${ }^{19}$ The relative normalizations of the Noether charges in both algorithms is not known a priori. In the approach of 64, 65] the construction of the Hamiltionian $H$ and the derivation the first law remain to be done. Therefore the normalization of the Noether charge has to be fixed by either comparing to the results of 61, 62] or by imposing that for the Einstein-Hilbert Lagrangian the Bekenstein-Hawking area law has the correct normalization.
} 
Now consider a static black hole in isotropic coordinates. The non-vanishing components of the binormal are $\varepsilon_{t r}=-\varepsilon_{r t}$, whereas the metric is diagonal. Therefore the contraction with the binormal yields $8 \pi \frac{\partial \mathcal{L}}{\partial R_{\mu \nu \rho \sigma}}=-\left(\varepsilon_{t r}\right)^{2} g^{t t} g^{r r}$. Now we use the explicit form of these expressions, $\varepsilon_{t r}=e^{g+f}, g^{t t}=-e^{-2 g}$ and $g^{r r}=e^{-2 f}$ with the result that

$$
\mathcal{S}=\frac{2 \pi}{8 \pi} \int_{\Sigma} \sqrt{h} d \Omega=\frac{A}{4} .
$$

Finally we mention some more results on entropy in higher derivative gravity. For Lagrangians which depend on derivatives of the Riemann tensor the entropy formula contains additional terms [62]. An alternative way of defining the entropy is provided by semiclassical Euclidean methods. These also predict deviations from the Bekenstein Hawking area law and the results are equivalent to those of the Minkowskian approach, in all cases where both methods apply [66]. Finally one can try to extend the definition of entropy to non-stationary black holes. This way it has been argued that the second law should be valid in higher derivative gravity [62], though there seems to be no complete proof. We refer to 667, 68] for recent reviews and more references concerning the laws of black hole mechanics.

\subsubsection{Extremal Black Holes as Supersymmetric Solitons}

Extremal black holes have very special properties and it turns out that these can be understood in terms of a symmetry principle, namely supersymmetry. After embedding gravity into extended supergravity, extremal black holes provide examples of supersymmetric solitons. We will consider the explicit example of the extremal Reissner-Nordstrøm black hole of EinsteinMaxwell theory, following the orginal work of [10, 69], but with the notation and conventions that we will use in chapter 4 to analyse more general black holes.

Let us first collect what is special about extremal black holes. We already mentioned that they saturate the mass bound defined by the existence of an event horizon. Therefore the mass is determined by the charge carried by the black hole. Next the surface gravity vanishes, and according to the thermodynamic interpretation this means that they have zero temperature and are stable against Hawking radiation. Note that this property makes them particularly interesting for finding a microscopic interpretation of the black hole entropy. Closer inspection reveals further particular properties. Consider for example the asymptotic geometry at the event horizon. In isotropic coordinates, where the horizon is located at $r=0$ the asymptotic metric is

$$
d s^{2}=-\frac{r^{2}}{Q^{2}} d t^{2}+\frac{Q^{2}}{r^{2}}\left(d r^{2}+r^{2} d \Omega^{2}\right) .
$$

This metric is known as the Bertotti-Robinson solution [70, 71].20 By switching to a new radial variable $\rho$, where $Q^{2} / \rho^{2}=r^{2} / Q^{2}$, or, alternatively, by computing the Weyl tensor one varifies that this geometry is conformally flat. Moreover the curvature scalar vanishes, so that only the traceless part of the Ricci tensor is non-trivial. By inspection of the metric one sees that spacetime factorizes into a two-sphere, parametrized by $(\phi, \theta)$ and a second surface parametrized by $(t, r)$. This second factor is two-dimensional Anti-de Sitter space, $A d S^{2}=S O(2,1) / S O(1,1)$. Both the sphere and the $A d S$-space have the same curvature radius and since the $A d S$-space has negative curvature the total curvature scalar is zero, as mentioned above.

Another special property of the extremal Reissner-Nordstrøm black hole is that it has a multi-centered generalization, i.e. there exist static configurations of black holes, which can be placed at arbitrary positions in space as long as their horizons do not overlapp and all their charges have equal sign. Then the gravitational attraction and electrostatic repulsion cancel precisely irrespective of the position. The corresponding metric belongs to the class of metrics which was discovered by Majumdar and Papapetrou [72, 73],

$$
d s^{2}=-H^{-2}(\vec{x}) d t^{2}+H^{2}(\vec{x}) d \vec{x}^{2},
$$

${ }^{20}$ Actually, this is one particular case of the class of metrics studied by Bertotti and Robinson. The class consists of all solutions of the Einstein-Maxwell theory with a covariantly constant electromagnetic field strength. The above solution is a special case with vanishing cosmological constant and without charged matter ('dust'). 
where $H(\vec{x})$ is a harmonic function with respect to the Laplacian $\Delta=\sum_{i=1}^{3} \partial_{x^{i}} \partial_{x^{i}}$. The most general choice of $H$ that does not lead to naked singularities is the multi-centered extremal Reissner-Nordstrøm solution [74]:

$$
H(\vec{x})=1+\sum_{i=1}^{N} \frac{M_{i}}{\left|\vec{x}-\vec{x}_{i}\right|},
$$

where $M_{i}, \vec{x}_{i}$ are the mass and position (of the event horizon) of the i-th black hole. Since no net forces act between the black holes the mass is additive,

$$
M=\sum_{i=1}^{N} M_{i}=\sum_{i=1}^{N} \sqrt{Q_{i}^{2}+P_{i}^{2}},
$$

where in the second equality we took a general, dyonic charge configuration with electric charges $Q_{i}$ and magnetic charges $P_{i}$. In this case the charges must have 'equal sign' in the sense that all the complex numbers $Q_{i}+i P_{i}$ must have the same phase.

We will now review how these special properties of the extremal Reissner-Nordstrøm black hole can be understood in terms of supersymmetry. First we should explain our use of the term 'soliton'. Usually a soliton is a static solution of the classical equations of motion that has finite energy and is regular everywhere. Sometimes one also includes the requirement that the soliton can be argued to be quantum mechanically stable. Very often solitons interpolate between two different vacua of the underlying theory. We refer to [75] for an overview of solitons and instantons in field theory.

In the context of gravity a less restrictive definition is adequate. Solitons are still required to be static finite energy solutions of the classical equations of motion.? 'Regular' is interpreted as 'having no naked singularities', so that solutions where singularities are covered by event horizons are admitted. For extremal black holes of extended supergravity theories one can give an argument in favour of their quantum mechanical stability that will be reviewed below. Finally, we will see that extremal black holes indeed interpolate between two distinct vacua of extended supergravity.

Next we have to explain what we mean by a 'supersymmetric' soliton. To do so we have to introduce the notion of a BPS state and review a bit of the representation theory of the supersymmetry algebra, see for example [76]. We consider four-dimensional $\mathcal{N}=2$ supersymmetry, because we are interested in four-dimensional theories and in four dimensions this is the most general case where BPS states can be defined. The supersymmetry algebra can be brought to the form

$$
\begin{aligned}
\left\{Q_{\alpha}^{i}, Q_{\beta}^{j+}\right\} & =2 P_{\mu} \sigma_{\alpha \beta}^{\mu} \delta^{i j} \\
\left\{Q_{\alpha}^{i}, Q_{\beta}^{j}\right\} & =2 Z \varepsilon_{\alpha \beta} \varepsilon^{i j}
\end{aligned}
$$

where we parametrized the supersymmetry generators by two Weyl spinors $Q_{\alpha}^{i}$. The indices $\alpha, \beta, . .=1,2$ are Weyl spinor indices, whereas $i, j, \ldots=1,2$ count the supercharges. We denote the hermitean conjugated charges by $Q_{\alpha}^{i+}$ and refrain from using dotted indices for the opposite chirality representation. As in all cases of extended supersymmetry, the algebra contains central charges, i.e. operators that commute with all generators of the algebra. In our case there is one complex central charge $Z$. It follows directly from the algebra that all massive representations satisfy a mass bound, which is given by the central charge,

$$
M \geq|Z| .
$$

Moreover states which saturate this bound,

$$
M=|Z|,
$$

\footnotetext{
${ }^{21}$ This generalizes to solutions with $p$ non-compact spatial isometries ( $p$-branes) by replacing 'finite energy' by 'finite energy per world volume'.
} 
have special properties. The structure of unitary representations is different for the cases $M>|Z|$ and $M=|Z|$. In the case $M=|Z|$ half of the supercharges act trivially, and therefore the multiplet is generated by $2^{\mathcal{N}}=2^{2}$ creation operators instead of $2^{2 \mathcal{N}}=2^{4}$ in the generic case. This is referred to as multiplet shortening, and the multiplets are called short and long multiplets, respectively. For short multiplets the mass is termined by the central charge. This relation cannot be changed by perturbative or non-perturbative corrections (assuming that the full theory is supersymmetric).

So far we have only considered the supersymmetry algebra and now turn to supersymmetric field theories. The fundamental fields in the Lagrangian can belong to either short or long representations. Two important short multiplets occuring in $\mathcal{N}=2$ Super-Yang-Mills theories and in $\mathcal{N}=2$ string compactifications are the short vector multiplet and the hypermultiplet. The on-shell degrees of freedom of a short vector multiplet are a (massive) vector, two Weyl fermions and a real scalar. A hypermultiplet contains two Weyl fermions and four real scalars. Whereas there is no 'long' version of the hypermultiplet, there is a long vector multiplet which contains the combined on-shell degrees of freedom of a short vector multiplet plus a hypermultiplet.

The short vector multiplet and the hypermultiplet have the same degrees of freedom as the corresponding massless multiplets. This is related to the two types of the Higgs mechanism that exist in $\mathcal{N}=2$ gauge theories, see for example [77. The scalar potential has flat directions which correspond to vacuum expectation values for either the scalars in short vector multiplets or to those in hypermultiplets. If one gives vacuum expectation values to scalars in vector multiplets in a generic way, then all charged massless vector multiplets become short massive vector multiplets and the gauge group is broken to its maximal torus. If one gives vacuum expectation values to scalars in hypermultiplets in a generic way then pairs of massless vector and hypermultiplets combine into long vector multiplets and the gauge group is broken completely, if sufficiently many hypermultiplets are present. These two generic kinds of flat directions constitute the Coulomb and the Higgs branch of the theory, respectively. Note that the two mechanisms tend to exclude one another. Once one is at a generic point in the Coulomb branch (Higgs branch) it is no longer possible to give a generic vacuum expectation value to scalars in hypermultiplets (vector multiplets). Depending on the precise field content there might be mixed branches where non-generic vacuum expectation values can be simultanously turned on for scalars both in vector and hypermultiplets, see for example [77 for more details.

We now turn to solitons and start with rigid supersymmetric theories. Like the fundamental fields in the Lagrangian the solitons of $\mathcal{N}=2$ Super-Yang-Mills theory can sit in short multiplets. For example the 't Hooft-Polyakov-monopoles of Super-Yang-Mills are in short hypermultiplets.22 This plays a prominent role in the Seiberg-Witten analysis of $\mathcal{N}=2$ gauge theories, see again [77 for more details. In non-supersymmetric gauge theories monopoles saturating the Bogomol'nyi mass bound are called Bogomol'nyi-Prasad-Sommerfield or BPS solitons. The special properties of such objects can be understood in terms of supersymmetry after extending the theory to an $\mathcal{N}=2$ supersymmetric theory. Therefore the terminology has been transferred to supersymmetric theories. In particular the supersymmetric mass bound is also called the BPS bound and states saturating it are called BPS states.

We now turn to $\mathcal{N}=2$ supergravity where we have to take into account that supersymmetry is realized as a local symmetry. Einstein-Maxwell theory can be naturally embedded into pure $\mathcal{N}=2$ supergravity by adding two Majorana spin $3 / 2$ fields, the gravitini. The gravitini $\psi_{\mu}^{i}$, the graviton (vielbein) $e_{\mu}{ }^{a}$ and the photon $A_{\mu}$ form the $\mathcal{N}=2$ supergravity multiplet. $(i=1,2$ is the internal $\mathcal{N}=2$ index, $\mu$ is a world index and $a$ is a tangent space index.) In this context the gauge field $A_{\mu}$ is usually called the graviphoton. In $\mathcal{N}=2$ supergravity the central charge transformations are local $U(1)$ symmetries, with the graviphoton as gauge field. As a consequence the central charge carried by a field configuration is related to its electric and magnetic charge by $Z=Q+i P$ (modulo a conventional phase) [78]. Thus the black hole mass bound $M=\sqrt{Q^{2}+P^{2}}$ translates into the supersymmetric one, $M=|Z|$.

Whereas it is obvious that the black hole mass bound should be interpretable as a su-

\footnotetext{
${ }^{22}$ Due to the existence of flat directions one is automatically in the Prasad-Sommerfield limit and the Bogomol'nyi bound of Yang-Mills theory can be understood as a supersymmetric mass bound.
} 
persymmetric mass bound in the supergravity theory, it is less obvious what we mean by a supersymmetric state or supersymmetric soliton in a locally supersymmetric theory. It is now useful to recall that local symmetries are just reduncancies in the parametrization of a theory whereas rigid (or global) symmetries are symmetries in the strict sense of the word, i.e. they lead to the identification of states, rather then just providing reparametrizations of the same state. Concerning local transformations one has to distinguish those which are strictly local (in the sense that the transformation parameter has compact support or vanishes fast) from those which asymptotically approach a rigid transformation (i.e. the transformation parameter approaches a constant). Thus local transformations in the broader sense include rigid transformation and local transformations in the narrow sense.

For a black hole supersymmetry transformations with a transformation parameter that approaches a constant at infinity are rigid transformations. They describe the collective modes of the corresponding field configuration. 23 This is obvious in the case of translations or rotations, which are, like the supersymmetry transformations, part of the supersymmetry algebra. Typically a soliton (or any other non-trivial field configuration) has less symmetry than the vacuum. For instance a black hole solution will never be translationally invariant. This is sometimes phrased in the way that the soliton 'breaks translational invariance', a somewhat trivial statement that should not be confused with spontanous symmetry breaking, which refers to properties of the vacuum. If the underlying theory (i.e. the Lagrangian) is translationally invariant, this will manifest itself in the degeneracy of all solutions that are related by translations. Such motions constitute the collective modes of the soliton. But there exist solitons which are more symmetric than a generic field configuration, and which share some of the symmetries of the vacuum. This is most easily visualized when thinking about rotations rather than translations. As we have seen above the stationary black holes of Einstein-Maxwell theory are either spherically symmetric or axisymmetric. The corresponding transformations do not generate new states but are isometries of the metric and the transformation parameters are Killing vectors.

The same phenomenon can happen with supersymmetry transformations. If one can find supersymmetry transformation parameters $\epsilon(x)$ such that a particular field configuration is invariant, one has the fermionic analogue of an isometry. The corresponding parameters are called Killing spinors. The prime example is the extremal Reissner Nordstrøm solution when embedded into $\mathcal{N}=2$ supergravity. The relevant field configuration is purely bosonic: Only the vielbein and the graviphoton are non-trivial whereas the gravitini are trivial, $\psi_{\mu}^{i}=0$. In order to prove the existence of Killing spinors one has to show that

$$
\left.\delta_{\epsilon(x)}\left(e_{\mu}^{a}(x), A_{\mu}(x), \psi_{\mu}^{i}(x)\right)\right|_{\text {extr. RN }}=0
$$

for some choice of the transformation parameter $\epsilon(x)$. Note that the supersymmetry transformation has to be evaluated on the background solution, since we are asking for an invariance of this particular field configuration. The invariance of the graviton and of the graviphoton is trivial, because they transform into fermionic quantities that vanish in the background. The non-trivial part is the variation of the gravitini,

$$
\left.\delta_{\epsilon(x)} \psi^{i}(x)\right|_{\text {extr. RN }}=0 .
$$

One can consider this as an equation for $\epsilon(x)$ and solve it with the result that one finds four Killing spinors. For later use it is instructive to proceed in a more general way, which is standard in the theory of solitons. The point is that the Killing spinor equation is first order in the bosonic background and therefore much easier to solve than the equations of motion. The same applies to the Bogomol'nyi equation of Yang-Mills-Higgs theory. One can therefore try to find supersymmetric solitons by taking the Killing equation as an equation for the supersymmetric background. In addition one should of course impose further symmetry properties in order to have a tractable problem. An ansatz for finding supersymmetric (multi) solitons is a general 'conformastatic' metric,

$$
d s^{2}=-e^{2 g(\vec{x})} d t^{2}+e^{2 f(\vec{x})} d \vec{x}^{2},
$$

\footnotetext{
${ }^{23}$ We refer to 755 for a detailed discussion of collective modes of solitons.
} 
which is static and has a conformally flat spatial part. In addition one requires that the gauge field is static. In a bosonic background the Killing spinor equation takes the form

$$
\left.\delta \psi_{\mu i}\right|_{\text {bosonic bg }}=D_{\mu} \epsilon_{i}=\nabla_{\mu} \epsilon_{i}-\frac{1}{4} F_{\rho \sigma}^{-} \gamma^{\rho} \gamma^{\sigma} \gamma_{\mu} \varepsilon_{i j} \epsilon^{j}=0,
$$

where $F_{\mu \nu}^{-}$is the antiselfdual part of the field strength and $\epsilon_{i}$ and $\epsilon^{i}$ are chiral projections of the two Majorana spinors that parametrize the supersymmetry transformations. ${ }^{24}$ In order to get an overview over the possible solutions one can analyse the integrability conditon

$$
D_{[\mu} D_{\nu]} \epsilon_{i}=0 .
$$

One class of solutions is obtained by imposing spherical symmetry and invariance under all $\mathcal{N}=2$ supertransformations. This corresponds to the maximal number of eight Killing spinors. Then the geometry is the Bertotti-Robinson geometry $A d S^{2} \times S^{2}$ with covariantly constant field strength. In the limit of infinite radius one recovers flat Minkowksi space. These are the only static, spherically symmetric and fully $\mathcal{N}=2$ supersymmetric field configurations.5 Since the field equations are automatically satisfied we will call them vacua. Observe that these are precisely the asymptotic geometries of the extremal Reissner-Nordstrøm black hole at the horizon and at infinity. Thus the black hole interpolates between two vacua of $\mathcal{N}=2$ supergravity.

Another class of solutions is found when imposing that the background is conformastatic and that the Killing spinor satisfies

$$
\epsilon_{i}+i \gamma_{0} \frac{\Sigma}{|\Sigma|} \varepsilon_{i j} \epsilon^{j}=0
$$

where $\Sigma$ is allowed to depend on the fields. ${ }^{26}$ In this case half of the parameters are fixed in terms of the others so that there are only four Killing spinors. Thus the solution is invariant under half of the supersymmetry transformations, as expected for a BPS soliton. It turns out that these solutions are precisely the Majumdar-Papapetrou solutions discussed above. They are the most general static supersymmetric solutions of the theory. The explicit form of the Killing spinor is

$$
\epsilon_{i}(\vec{x})=H^{-1 / 2}(\vec{x}) \epsilon_{i}(\infty)
$$

where the asymptotic value of the spinor is constrained by

$$
\epsilon_{i}(\infty)+i \gamma^{0} \frac{Z}{|Z|} \varepsilon_{i j} \epsilon^{j}(\infty)=0 .
$$

$Z$ is the central charge and $H$ is the harmonic function of the Majumdar-Papapetrou solution. The relation between the asymptotic transformation parameters can be directly understood from the supersymmetry algebra. If the soliton is a supersymmetric state, then the asymptotic transformation parameters must be null eigenvectors of the Bogomol'nyi matrix, which is the matrix of all supersymmetry anticommutators, evaluated in the background. This observation allows one to systematically construct supersymmetric solitons, such as $p$-branes in more complicated supergravity theories, see for example [79].

Due to the invariance under half of the supertransformations, the number of fermionic collective modes of the supersymmetric Reissner-Nordstrøm black hole is reduced by a factor of $1 / 2$, so that there are 4 instead of 8 . By supertransformations one can generate the corresponding 'half hypermultiplet'. Such a multiplet contains a fermion and two real scalars. Generically the multiplet carries some non-vanishing quantum number and therefore it is not CP-selfconjugate. Then one has to add the CP-conjugated half hypermultiplet to restore CPT-invariance and

\footnotetext{
${ }^{24}$ As already mentioned we follow [10, 69], but use our own conventions, which are explained in chapter 4 .

${ }^{25}$ This will be derived in chapter 1 for a more general class of $\mathcal{N}=2$ supergravity Lagrangians. One can show an even stronger result [19]: the Bertotti-Robinson geometry is the only stationary and $N=2$ supersymmetric field configuration for the class of Lagrangians discussed in chapter 1 .

${ }^{26}$ See again chapter 1 for the details.
} 
this way one obtains a hypermultiplet. In our case the black hole solution carries electric or magnetic charge and one gets a full hypermultiplet by adding the black hole of opposite charge and its supersymmetry partners. Thus the extremal Reissner-Nordstrøm black hole belongs to a hypermultiplet, like the monopoles and dyons of $\mathcal{N}=2$ Super-Yang-Mills theories.

In conclusion we have seen that supersymmetry accounts for all the particular features of the extremal Reissner-Nordstrøm black hole. This manifests itself nicely when expressing the metric, the mass and the entropy in terms of the central charge,

$$
\begin{gathered}
d s^{2}=-\left(1+\frac{|Z|}{r}\right)^{-2} d t^{2}+\left(1+\frac{|Z|}{r}\right)^{2}\left(d r^{2}+r^{2} d \Omega^{2}\right), \\
M=|Z|, \quad \mathcal{S}=\pi|Z|^{2} .
\end{gathered}
$$

As a further application we would like to mention that the above methods can also be used to prove statements about non-supersymmetric theories of gravity. Using the embedding into a supersymmetric theory as a mere tool one can prove, under certain technical assumptions such as the dominant energy condition, that the mass of a static, asymptotically flat space-time in Einstein gravity coupled to matter is bounded by its total charge through

$$
M \geq|Q|
$$

and that all static space-times saturating the bound posess four Killing spinors and therefore belong to the Majumdar-Papapetrou class 69.

We already mentioned that the method that we explained here for the extremal ReissnerNordstrøm black hole can also be used to systematically construct supersymmetric solitons of supergravity theories in various dimensions. The knowledge of these supergravity solitons was crucial for the study of string dualities and for establishing the new M-theory paradigm that all five perturbatively consistent string theories, together with eleven-dimensional supergravity, are asymptotic expansions of one underlying theory 80, 81.

\subsubsection{Outlook}

In the following chapters we will extend and connect all the topics introduced here. Instead of pure $\mathcal{N}=2$ supergravity we will consider the generic low energy effective theory of a $\mathcal{N}=2$ string compactification, namely $\mathcal{N}=2$ supergravity coupled to a model-dependent number of abelian vector multiplets and neutral hypermultiplets. Since supersymmetry does not allow gauge-neutral couplings of vector and hypermultiplets [16], one can find solutions of the equations motion where the hypermultiplets are trivial. Since this class of solutions is already very rich, and contains a variety of charged black holes, we will neglect hypermultiplets in the following.77 In our discussion we will include a particular class of higher curvature terms, which are quadratic in the Riemann tensor. We will then study generalizations of the extremal Reissner-Nordstrøm black hole in these theories and in particular derive an entropy formula for them according to the proposal of Wald.

After embedding supergravity into string theory it is possible to lift some of our solutions to ten-dimensonial string theory or eleven-dimensional M-theory. By counting the collective modes of the higher dimensional solitons one gets a microscopic entropy that can be compared to the macroscopic one. Both expressions agree and we will see that this depends on various subtleties like the proper treatment of the higher curvature terms.

\footnotetext{
27 More recently we have shown that in a stationary space-time with residual supersymmetry as specified in (2.70) the hypermultiplet scalars are automatically constant [19].
} 


\section{Chapter 3}

\section{Four-Dimensional $\mathcal{N}=2$ Supergravity}

In this chapter we review $\mathcal{N}=2$ supergravity coupled to an arbitrary number of abelian vector multiplets. This is the part of the low energy effective field theory of a $\mathcal{N}=2$ string compactification that we will need for the construction of black hole solutions. The discussion is model independent, concrete compactifications will be discussed later.

The approach that we will describe is based on the so-called superconformal multiplet calculus and was developed in [82, 15, 83, 16]. It has the advantage that it provides an off-shell formulation of the theory and that one can include a particular class of curvature squared terms, which are encoded in the Weyl multiplet. Both properties will be important when we study black hole solutions later. The whole vector multiplet Lagrangian is encoded in a single function of the vector multiplets, called the prepotential. The scalar sigma-model must take values in a special Kähler manifold. The structure of the Lagrangian and the role of the prepotential can be understood in terms of special Kähler geometry or special geometry, for short [33, 17, 16]. We will also see that symplectic transformations, which generalize electric - magnetic duality rotations, play an important role. They are intimately related to the special Kähler geometry of the scalar sector, because $\mathcal{N}=2$ vector multiplets contain both gauge fields and scalars.

The two crucial ingredients of the approach are the construction of extended conformal supergravity as a gauge theory of the superconformal group 84 and the gauge equivalence of conformal and Poincaré supergravity. It turns out that it is much easier to find off-shell realizations of the $\mathcal{N}=2$ superconformal algebra. In particular one can find smaller off-shell multiplets: Whereas the standard $\mathcal{N}=2$ Poincaré supergravity multiplet 85, 86, 82 has $40+40$ off-shell degrees of freedom, the minimal representation of $\mathcal{N}=2$ conformal supergravity has only $32+32$. Moreover the minimal representation is reducible and decomposes into the Weyl multiplet, with $24+24$ degrees of freedom and a vector multiplet wit $8+8$.

The supersymmetry transformation rules have a simpler form in the superconformal theory and one can in a systematic way derive these rules and the Lagrangian starting from a gauge theory associated with the $\mathcal{N}=2$ superconformal algebra. By adding multiplets which act as compensators one can construct $\mathcal{N}=2$ superconformal Lagrangians in such a way that they reduce upon partial gauge fixing to $\mathcal{N}=2$ super Poincaré Lagrangians.

The plan of this chapter is as follows. We first illustrate how gauge equivalence works using a non-supersymmetric toy example. Then we review the construction of the relevant superconformal off-shell multiplets, the superconformal action and symplectic transformations. Finally we discuss the transition to Poincaré supergravity and special geometry. The conventions are those of [90, 91], which are somewhat different from those in the original papers quoted above. Our presentation has profited a lot from the nice review contained in [92]. Reviews of $\mathcal{N}=2$

\footnotetext{
${ }^{1}$ It is known that smaller off-shell representations of $\mathcal{N}=2$ Poincareé supergravity exist: There are two $32+32$ representations [87, 88] and a $24+24$ representation [89]. In the first two cases an action is also known. But for our purposes we need to know matter couplings and $R^{2}$-couplings.
} 
conformal supergravity are 93,94 .

At the end of the chapter we make contact with the on-shell formulation of $\mathcal{N}=2$ supergravity developed in [95, 96], which is also called the intrinsic or geometric formulation. Reviews which also contain more references on this approach to $\mathcal{N}=2$ supergravity are 97,98 . Our treatment of black holes in the next chapter will be based on the off-shell formulation, but since most of the literature on $\mathcal{N}=2$ black holes is based on the on-shell formulation it is important for us to know how the two formulations are related. The advantages and disadvantages of the two formalisms are complementary. The superconformal off-shell formulation allows to include $R^{2}$-terms, but is tied to a parametrization of the scalar manifold in terms of so-called special coordinates. The geometric on-shell formulation does not depend on special coordinates and one can describe the theory in terms of a section over the scalar manifold instead of using a prepotential. This is relevant when constructing the most general gauged $\mathcal{N}=2$ supergravity Lagrangian, but does not lead to more general couplings of abelian vector multiplets. The reason why we prefer the superconformal formulation is that the geometric formulation only provides the on-shell supersymmetry transformation rules and that it has not been worked out how to include $R^{2}$-terms.

\subsection{Gauge Equivalence}

Before entering into the details of the superconformal formalism we illustrate the concept of gauge equivalence in a simple example. The Einstein-Hilbert action can be obtained from the action of a scalar field coupled to conformal gravity by partial gauge fixing of the conformal symmetries. This is a standard example for gauge equivalence. We follow [92, 99]. We try to focus on the line of thought and omit several details, which can be found either in the references or, for the more complicated $\mathcal{N}=2$ supersymmetric generalization, in later sections.

First we recall the Poincaré Lie algebra iso $(1,3)$ :

$$
\left[P_{a}, M_{b c}\right]=2 P_{[b} \eta_{c] a}, \quad\left[M_{a b}, M_{b c}\right]=2 \eta_{[a[c} M_{b] d]},
$$

where $a, b, \ldots=0, \ldots, 3$ are flat indices (tangent space indices). $P_{a}$ generate the translations and $M_{b c}$ generate the Lorentz transformations. This is extended to the Lie algebra of conformal transformations $\mathrm{su}(2,2) \simeq \mathrm{so}(2,4)$ by adding the generators $K_{a}$ of special conformal transformations and $D$ of dilatations:

$$
\left[K_{a}, M_{b c}\right]=2 K_{[b} \eta_{c] a}, \quad\left[P_{a}, K_{b}\right]=\eta_{a b} D-2 M_{a b}, \quad\left[D, P_{a}\right]=P_{a}, \quad\left[D, K_{a}\right]=-K_{a} .
$$

Finite special conformal transformations are singular on hypersurfaces in Minkowski space, but we only consider infinitesimal transformations here.

The first step is to construct a gauge theory of the conformal group. One introduces connections $e_{\mu}{ }^{a}, \omega_{\mu}^{a b}, f_{\mu}^{a}, b_{\mu}$ corresponding to $P_{a}, M_{a b}, K_{a}, D$. Here $\mu, \nu=t, x, y, z$ are space-time indices (curved indices, world indices). The corresponding curvatures are denoted by $R(P)_{\mu \nu}^{a}$, etc. At this point the superconformal tranformations have been introduced as purely internal transformations, i.e. they are space-time dependent but do not act on space-time, but rather on a bundle over space-time.

The next step is to interpret the translational connection $e_{\mu}{ }^{a}$ as the vielbein 2 , the local translations as general coordinate transformations of space-time and the local Lorentz transformations as acting on the tangent bundle of space-time, i.e. as local Lorentz transformations of the vielbein. As a consequence the Lorentz connection $\omega_{\mu}^{a b}$ is identified with the spin connection.

In order to make these identifications one has to impose so-called conventional constraints on the conformal curvatures. Such constraints have to be algebraic in order that they do not impose dynamical equations on the field. Moreover they have to be consistent with the underlying algebra. In the case of conventional constraints this is guaranteed because one imposes relations that can be realized by redefinitions of the connections. When imposing such

\footnotetext{
${ }^{2}$ This means that we require that $e_{\mu}^{a}$ is invertible.
} 
conditions as constraints, the Bianchi identities become non-trivial and it might be necessary to modify the transformation rules of the remaining independent fields in order to have consistent realization of the algebra.

In the case at hand the constraints have an obvious physical content. In general relativity we know that the spin connection $\omega_{\mu}^{a b}$ can be expressed in terms of the vielbein $e_{\mu}{ }^{a}$. This has to be implemented by the constraints. More generally the constraints have to be chosen such that all conformal transformations become transformations acting in space-time. For example the local translations (P-transformations) have to become general coordinate transformations on all fields.

All this can be implemented by imposing the conventional constraints

$$
R(P)_{\mu \nu}^{a}=0, \quad e_{b}^{\nu} R(M)_{\mu \nu}^{a b}=0 .
$$

These relations can be solved for the dependent connections $\omega_{\mu}^{a b}$ and $f_{\mu}{ }^{a}$ :

$$
\begin{aligned}
\omega_{\mu}^{a b} & =-2 e^{\nu[a} \partial_{[\mu} e_{\nu]}^{b]}-e^{\nu[a} e^{b] \sigma} e_{\mu c} \partial_{\sigma} e_{\nu}^{c}-2 e_{\mu}{ }^{[a} e^{b] \nu} b_{\nu} \\
f_{\mu}{ }^{a} & =\frac{1}{2}\left(R_{\mu \nu}-\frac{1}{6} g_{\mu \nu} R\right) e^{\nu a}
\end{aligned}
$$

The spin-connection $\omega_{\mu}^{a b}$ differs from the standard spin-connection of general relativity by the additional term involving the dilatational connection $b_{\mu}$. This additional term is needed to make the Riemann tensor covariant with respect to dilatations.

Consider now the case of a massless scalar field, which is taken to be $K$-invariant and to have Weyl weight (dilatational or $D$-weight) $w=1$. The minimal coupling to conformal gravity is given by the Lagrangian

$$
\mathcal{L}=-e \phi D^{\mu} D_{\mu} \phi
$$

where $e=\operatorname{det}\left(e_{\mu}{ }^{a}\right)$ and $D_{\mu}$ is the conformal covariant derivative. Under special conformal transformations the dilatational gauge field $b_{\mu}$ transforms as

$$
\delta_{K} b_{\mu}=\Lambda_{K \mu},
$$

where $\Lambda_{K \mu}$ are the transformation parameters of the $K$-transformation. The first and second covariant derivative of $\phi$ are:

$$
\begin{aligned}
& D_{\mu} \phi=\partial_{\mu} \phi-b_{\mu} \phi, \\
& D_{\mu} D^{a} \phi=\left(\partial_{\mu}-2 b_{\mu}\right) D^{a} \phi-\omega_{\mu}^{a b} D_{b} \phi+f_{\mu}{ }^{a} \phi .
\end{aligned}
$$

In the first line the term $-b_{\mu}$ provides the covariantization with respect to dilatations, because $\phi$ has $w=1$. In the second line $D^{a} \phi$ is not invariant under $K$-transformations, because it contains $b_{\mu}$. Therefore the extra term involving the $K$-connection $f_{\mu}{ }^{a}$ is necessary for making the second derivative covariant. This illustrates a general principle: In theories with several gauge symmetries and corresponding connections the structure of covariant derivatives can be systematically determined by considering the transformation properties of the object one differentiates, see appendix $\mathrm{G}$.

The Lagrangian (3.5) is conformally invariant and in particular invariant under special conformal transformations. Since $b_{\mu}$ is the only independent gauge field that transforms under $K$, one knows a priori that the $b_{\mu}$-terms in (3.5) must cancel out when expressing the dependent connections in terms of the independent ones. Explicitly one finds:

$$
\mathcal{L}=-e \phi \mathcal{D}^{\mu} \mathcal{D}_{\mu} \phi-e f_{\mu}{ }^{\mu} \phi^{2},
$$

where $\mathcal{D}_{\mu} \phi=\partial_{\mu} \phi$ is the covariant derivative with respect to general coordinate transformations. By partial integration and elimination of the $K$-connection in terms of independent quantities one gets

$$
\mathcal{L}=e \partial_{\mu} \phi \partial^{\mu} \phi-\frac{1}{6} e \phi^{2} R
$$


When gauge fixing the $D$-transformations by imposing $\phi=\sqrt{6} /(\sqrt{2} \kappa)$ we get the EinsteinHilbert action

$$
\mathcal{L}=-e \frac{1}{2 \kappa^{2}} R
$$

We will call the special parametrization of a conformal theory where only the Poincaré symmetry is manifest the Poincaré frame.

Conversely one can start with the Einstein-Hilbert action and add a scalar with suitable transformation properties to get the conformally invariant version of the Einstein-Hilbert action. The scalar acts as a compensator in the sense that it is tailor-made to compensate for the noninvariance of the Einstein-Hilbert action under conformal transformations. Since one adds new symmetries and new degrees of freedom in a balanced way, the total number of on-shell degrees of freedom remains unchanged, because the new degrees of freedom can be gauged away. The two actions are said to be gauge equivalent.

Note that we got the Einstein-Hilbert action by starting from a conformal matter action. There is no explicit term involving the conformal curvatures but the Einstein-Hilbert term is hidden in the minimal coupling. Similarly the $\mathcal{N}=2$ supergravity action can be obtained from one minimally coupled conformal vector multiplet (together with a second compensating multiplet).

Explicit curvature terms can also be added to the conformal action. They are not related to the Einstein-Hilbert term, but play a different role. Gauge invariant curvature terms in the conformal action are quadratic in the conformal curvatures and produce additional higher derivative terms in the Poincaré action. In particular one gets the Weyl action (2.4). In $\mathcal{N}=2$ supergravity we will include terms quadratic in the Weyl multiplet in the superconformal action and this way we will obtain in the Poincaré frame additional curvature terms, including terms quadratic in the Weyl tensor.

\subsection{Superconformal Multiplets}

\subsubsection{The Superconformal Algebra}

We now turn to the technical details of the superconformal formalism. In this section we review the $\mathcal{N}=2$ superconformal algebra and introduce the so-called chiral notation.

We already displayed the relations of the Poincaré and of the conformal Lie algebra in (3.1) and (3.2). The $\mathcal{N}$-fold extended superconformal algebra $\operatorname{su}(2,2 \mid \mathcal{N})$ is a supersymmetric extension of the conformal Lie algebra by adding $2 \mathcal{N}$ Majorana supercharges.

Let us start with the Poincaré superalgebra, which contains $\mathcal{N}$ Majorana supercharges $q^{i}$, $i=1, \ldots, \mathcal{N}$, satisfying the anticommutation relations [100]

$$
\left\{q^{i}, q^{j}\right\}=2 \gamma^{a} P_{a} \delta^{i j}+Z^{i j},
$$

where $Z^{i j}$ is a complex antisymmetric matrix of central charges. In the absence of central charges the algebra is invariant under the automorphism group (also called $R$-symmetry group) $U(\mathcal{N})_{R} \simeq S U(\mathcal{N})_{R} \times U(1)_{R} \cdot{ }^{3}$ In the presence of central charges the automorphism group is reduced to $U S p(\mathcal{N})=U(\mathcal{N}) \cap S p(\mathcal{N}, \mathbb{C})$. Since the supercharges are Majorana spinors, the action of $U(\mathcal{N})_{R}$ is chiral and the positive (left) and negative (right) chirality components transform in the fundamental and antifundamental representation, respectively.

It turns out to be useful to adopt the so-called chiral notation, which amounts to keeping track of spinor chiralities through writing the $S U(\mathcal{N})_{R}$ index as an upper or lower index 101, 102. Thus, upper and lower $S U(\mathcal{N})_{R}$ indices are correlated with a fixed spinor chirality and with either the fundamental or antifundamental representation. Note that depending on the spinor an upper index might be associated with left or with right chirality. The assignements are listed in various tables in this chapter. As a consequence $S U(\mathcal{N})_{R}$ indices are raised and lowered by complex conjugation.

\footnotetext{
${ }^{3}$ There is one exception: For $\mathcal{N}=4$ the automorphism group is $S U(4)_{R}$ and not $U(4)_{R} 100$.
} 
The chiral projections of the supercharges $q^{i}$ are:

$$
Q^{i}:=\frac{1}{2}\left(\mathbb{I}+\gamma_{5}\right) q^{i}, \quad Q_{i}:=\frac{1}{2}\left(\mathbb{I}-\gamma_{5}\right) q^{i} .
$$

Remember that the chiral projections of a Majorana spinor are not independent of each other. In the following we will omit several commutators which do not contain independent information. $\bar{\psi}$ denotes the Dirac conjugate of the spinor $\psi$. For Majorana spinors, such as the $Q$ - and $S$-supercharges, the Dirac conjugate equals the Majorana conjugate. We refer to appendix A for an overview of our spinor conventions.

The $\mathcal{N}$-fold extended superconformal algebra has twice as many fermionic generators as the $\mathcal{N}$-fold extended super Poincaré algebra [100]. The supercharges already present in the super Poincaré algebra are called $Q$-supercharges $Q^{i}, Q_{i}$, whereas the additional supercharges are called special supercharges or $S$-supercharges $S^{i}, S_{i}$. Both kinds of supercharges transform as Lorentz spinors:

$$
\left[M_{a b}, Q^{i}\right]=\frac{1}{2} \sigma_{a b} Q^{i}, \quad\left[M_{a b}, S^{i}\right]=\frac{1}{2} \sigma_{a b} S^{i} .
$$

Closure of the algebra (see 3.18) requires to include the $R$-symmetry algebra $U(\mathcal{N})_{R} \simeq$ $S U(\mathcal{N})_{R} \times U(1)_{R}$ into the superconformal algebra as well. The generators are denoted by $V_{\Lambda}$ and $A$, respectively. We now specialize to the case $\mathcal{N}=2$. In chiral notation the $S U(2)_{R}$ transformation rules read:

$$
\begin{array}{ll}
{\left[V_{\Lambda}, Q\right]^{i}=i\left(\sigma_{\Lambda}\right)^{i}{ }_{j} Q^{j},} & {\left[V_{\Lambda}, Q\right]_{i}=i\left(\sigma_{\Lambda}\right)_{i}{ }^{j} Q_{j},} \\
{\left[V_{\Lambda}, S\right]^{i}=i\left(\sigma_{\Lambda}\right)^{i}{ }_{j} S^{j},} & {\left[V_{\Lambda}, S\right]_{i}=i\left(\sigma_{\Lambda}\right)_{i}{ }^{j} S_{j},}
\end{array}
$$

where the generators are normalized as $\left[V_{\Lambda}, V_{\Sigma}\right]=-2 \epsilon_{\Lambda \Sigma}{ }^{\Xi} V_{\Xi}(\Lambda, \Sigma, \Xi=1,2,3)$.

Under dilatations and $U(1)_{R}$ transformations the supercharges transform as follows:

$$
\begin{array}{ll}
{\left[D, Q^{i}\right]=\frac{1}{2} Q^{i},} & {\left[A, Q^{i}\right]=-\frac{i}{2} Q^{i},} \\
{\left[D, S^{i}\right]=\frac{1}{2} S^{i},} & {\left[A, S^{i}\right]=\frac{i}{2} S^{i} .}
\end{array}
$$

The $U(1)_{R}$ transformations are chiral and $S_{i}, Q_{i}$ have the opposite charge (but the same dilatational weight).

The fermionic generators are related by $P_{a}$ and $K_{a}$ :

$$
\left[K_{a}, Q^{i}\right]=\gamma_{a} S^{i},\left[P_{a}, S^{i}\right]=\frac{1}{2} \gamma_{a} Q^{i} .
$$

The anticommutators of the $Q$ - and $S$-supercharges close into translations and special conformal transformations, respectively:

$$
\begin{aligned}
& \left\{Q^{i}, \bar{Q}_{j}\right\}=-\left(\mathbb{I}-\gamma_{5}\right) \gamma^{a} P_{a} \delta_{j}^{i}, \\
& \left\{S^{i}, \bar{S}_{j}\right\}=-\frac{1}{2}\left(\mathbb{I}+\gamma_{5}\right) \gamma^{a} K_{a} \delta_{j}^{i} .
\end{aligned}
$$

The mixed anticommutators yield other bosonic generators:

$$
\left\{Q^{i}, \bar{S}_{j}\right\}=\frac{1}{2}\left(\mathbb{I}-\gamma_{5}\right)\left(2 \sigma^{a b} M_{a b}+D-i A-2 V_{j}^{i}\right) .
$$

Finally we have a complex central charge $Z$,

$$
\left\{Q^{i}, \bar{Q}^{j}\right\}=\frac{1}{2}\left(\mathbb{I}-\gamma_{5}\right) \varepsilon^{i j} Z,
$$

where $\varepsilon_{i j}$ is the antisymmetric tensor in $i, j=1,2$.

\footnotetext{
${ }^{4}$ The Lorentz generators $\sigma_{a b}$ are defined in appendix A.
} 


\subsubsection{The Weyl Multiplet}

In this section we review the construction of the minimal off-shell representation of $\mathcal{N}=2$ conformal supergravity, the so-called Weyl or superconformal gauge multiplet 82, 15, 86, , 90, 92.

The first step in constructing $\mathcal{N}=2$ conformal supergravity is to build a superconformal gauge theory in which all generators of the algebra act as internal symmetries. This is in principle a straightforward procedure: Starting from the algebra one considers space-time dependent symmetry transformations and introduces gauge fields (connections). The gauge fields associated with translations $(P)$, Lorentz transformations $(M)$, dilatations $(D)$, special conformal transformations $(K), S U(2)$ and $U(1)$ transformations $(V, A)$ and $Q$ - and $S$ supertransformations are denoted by $e_{\mu}{ }^{a}, \omega_{\mu}^{a b}, b_{\mu}, f_{\mu}{ }^{a}, \mathcal{V}_{\mu}{ }^{i}{ }_{j}, A_{\mu}, \psi_{\mu}{ }^{i}, \phi_{\mu}{ }^{i}$. The $S U(2)$ gauge field $\mathcal{V}_{\mu}{ }^{i}{ }_{j}$ is antihermitean and traceless:

$$
\begin{aligned}
\mathcal{V}_{\mu j}^{i}+\mathcal{V}_{\mu j}{ }^{i} & =0 \\
\mathcal{V}_{\mu i}^{i}{ }^{i} & =0
\end{aligned}
$$

The (space-time) dependent transformation parameters corresponding to the transformations $T=P, M, D, K, Q, S, V, A$ are: $\xi^{a}, \varepsilon^{a b}, \Lambda_{D}, \Lambda_{K}^{a}, \varepsilon^{i}, \eta^{i}, \Lambda_{V j}^{i}, \Lambda_{A}$. The chirality properties and the Weyl and $U(1)$ weights of the gauge fields and transformation parameters are listed in table 3.1 .

The $Q \bar{Q}$ anticommutator (3.19) closes into the central charge and $Q$-transformations are realized as local transformations. Therefore the central charge transformations are local as well and we have to introduce a gauge field for them. This gauge field is not part of the Weyl multiplet but sits instead in a separate vector multiplet. Therefore the central charge gauge transformations can be treated as additional abelian gauge symmetries. This is consistent with the algebra because $Z$ commutes with all other generators.

The covariant derivative with respect to all superconformal transformations is

$$
D_{\mu}:=\partial_{\mu}-\sum_{T} \delta\left(h_{\mu}(T)\right)
$$

where the sum runs over all superconformal generators $T$. The covariantization of a derivative with respect to a transformation $T$ works by adding a gauge transformation with the gauge field $h_{\mu}(T)$ as parameter, see appendix C. Some of the gauge fields $h_{\mu}(T)$ appearing in the covariant derivative differ from the fields $e_{\mu}^{a}, \ldots$ listed above by normalization factors, see table 3.2 .

For later use we introduce another covariant derivative $\mathcal{D}_{\mu}$, which is covariant with respect to the bosonic $P, M, D, A, V$-transformations (and, if present, gauge transformations), but which does not include covariantization terms with repect to the fermionic $Q, S$ - transformations and with respect to the $K$-transformations.

Next one has to calculate the associated field strengths (curvatures) $R_{\mu \nu}(T)$ and to write down the corresponding transformation rules. Note that the notation $R_{\mu \nu}(T)$ is schematic, since most of the curvatures carry additional indices. When refering to a specific field strength we will always display all its bosonic indices (in practice Lorentz and $S U(2)$ indices) while surpressing the fermionic indices on the $Q$ - and $S$-field strengths. The explicit expressions for the field strengths are rather involved. Later we we will have to use modified field strengths, which will be introduced below. Therefore we will not need the explicit expressions for the $R_{\mu \nu}(T)$, which can be found in reference [82.

The second step is to rebuild this superconformal gauge theory into a theory of (conformal) supergravity. This means that the conformal symmetries and the supertransformations must be realized as space-time symmetries rather than as internal ones. In particular, local translations must be identified with local coordinate transformations and local Lorentz transformations must act as local rotations on the tangent bundle of space-time, whereas $Q$ supertransformations must close into general coordinate transformations, modulo other symmetries. As in the non-supersymmetric example discussed earlier the necessary identifications 
can be achieved by imposing conventional constraints. Since the constraints are not invariant under supersymmetry the dependent gauge fields that one obtains by solving the constraints transform differently from the original independent fields. In order to make the various field strengths $R_{\mu \nu}(T)$ covariant with respect to the modified transformation rules, additional terms have to be added. The new covariant curvatures are denoted $\widehat{R}_{\mu \nu}(T)$. The Bianchi identities are likewise modified. They are no longer identities in the literal sense, but become non-trivial equations, whose consistency has to be checked. All these changes can be fine-tuned in such a way that the commutator of two supersymmetry transformations closes into a general coordinate transformation. This requires the introduction of auxiliary fields, which together with the independent gauge fields form a superconformal multiplet, called the Weyl multiplet. It is the basic multiplet of the theory, because it describes the gravitational degrees of freedom.

One is interested in imposing as many constraints as possible in order to find a minimal representation of $\mathcal{N}=2$ conformal supergravity, i.e. a representation with the minimal number of degrees of freedom. The constraints are not completely fixed by the above requirements. However a suitable set of conventional constraints is known:

$$
\begin{aligned}
& R_{\mu \nu}(P)=0 \\
& \gamma^{\mu}\left(\widehat{R}_{\mu \nu}(Q)^{i}+\sigma_{\mu \nu} \chi^{i}\right)=0 \\
& e_{b}{ }^{\nu} \widehat{R}_{\mu \nu}(M)_{a}{ }^{b}-i \widehat{\widehat{R}}_{\mu a}(A)+\frac{1}{8} T_{a b i j} T_{\mu b}^{i j}-\frac{3}{2} D e_{\mu a}=0 .
\end{aligned}
$$

We denote the dual tensor of $R$ by $\widetilde{R}$. Note that we use a non-standard definition for the dual tensor, see appendix C. The auxiliary fields consist of an antiselfdual antisymmetric Lorentz tensor $T_{a b}^{i j}$, an $S U(2)$ doublet of Majorana spinors $\chi^{i}$ and a real scalar field $D$. Note that the tensor field is antisymmetric in its $S U(2)$ indices and, hence, is an $S U(2)$ singlet. In Minkowski space the selfdual and antiselfdual apart of an antisymmetric tensor are related by complex conjugation:

$$
\left(T_{a b i j}\right)^{*}=T_{a b}^{i j} .
$$

For later use we introduce

$$
T_{a b}^{+}=T_{a b i j} \varepsilon^{i j}, \quad T_{a b}^{-}=T_{a b}^{i j} \varepsilon_{i j} .
$$

Since the $S U(2)$ antisymmetric tensor is normalized by $\varepsilon_{i j} \varepsilon^{i j}=2$, the inverse relations read

$$
T_{a b i j}=\frac{1}{2} T_{a b}^{+} \varepsilon_{i j}, \quad T_{a b}^{i j}=\frac{1}{2} T_{a b}^{-} \varepsilon^{i j} .
$$

We next list the explicit expressions for the following modified field strengths:

$$
\begin{aligned}
\widehat{R}_{\mu \nu}(Q)^{i}= & 2 \mathcal{D}_{[\mu} \psi_{\nu]}^{i}-\gamma_{[\mu} \phi_{\nu]}^{i}-\frac{1}{4} \sigma^{a b} T_{a b}^{i j} \gamma_{[\mu} \psi_{\nu] j}, \\
\widehat{R}_{\mu \nu}(A)= & 2 \partial_{[\mu} A_{\nu]}-i\left(\frac{1}{2} \bar{\psi}_{[\mu}^{i} \phi_{\nu] i}+\frac{3}{4} \bar{\psi}_{[\mu}^{i} \gamma_{\nu]} \chi_{i}-\text { h.c. }\right), \\
\widehat{R}_{\mu \nu}(V)^{i}{ }_{j}= & 2 \partial_{[\mu} \mathcal{V}_{\nu] j}^{i}+\mathcal{V}_{[\mu}^{i} \mathcal{V}_{\nu] j}^{k} \\
& \left.+\left(2 \bar{\psi}_{[\mu}^{i} \phi_{\nu] j}-3 \bar{\psi}_{[\mu}^{i} \gamma_{\nu]} \chi_{j}-\text { (h.c. } ; \text { traceless }\right)\right) . \\
\widehat{R}_{\mu \nu}(M)^{a b}= & 2 \partial_{[\mu} \omega_{\nu]}^{a b}-2 \omega_{[\mu}^{a c} \omega_{\nu]}^{c b}-4 f_{[\mu}^{[a} e_{\nu]}^{b]}+\left(\bar{\psi}_{[\mu}^{i} \sigma^{a b} \phi_{\nu] i}+\text { h.c. }\right) \\
& \frac{1}{2} \bar{\psi}_{[\mu}^{i} T_{i j}^{a b} \psi_{\nu}{ }^{j}-\frac{3}{2} \bar{\psi}_{[\mu}^{i} \gamma_{\nu]} \sigma^{a b} \chi^{i}-\bar{\psi}_{[\mu}^{i} \gamma_{\nu]} \widehat{R}^{a b}(Q)_{i}+\text { h.c. } .
\end{aligned}
$$

By '-(h.c.; traceless)' we denote the projection of a product of spinors in $S U(2)$ doublets onto the antihermitean and traceless part. The explicit definition is

$$
\bar{\eta}^{i} \epsilon_{j}-\text { (h.c.; traceless) }=\bar{\eta}^{i} \epsilon_{j}-\bar{\eta}_{j} \epsilon^{i}-\frac{1}{2} \delta_{j}^{i}\left(\bar{\eta}^{k} \epsilon_{k}-\bar{\eta}_{k} \epsilon^{k}\right) \text {. }
$$

\footnotetext{
${ }^{5}$ To be precise, the commutator of two $Q$-supertransformations yields a so-called covariant general coordinate transformation plus further symmetry transformations. This is discussed below equation 3.43 .
} 
The other modified field strengths can be found in [82].

The constraints can be used to express the gauge fields of local Lorentz transformations $\omega_{\mu}^{a b}$, of special conformal transformations $f_{\mu}^{a}$ and of $S$-supertransformations $\phi_{\mu}^{i}$ in terms of the other gauge fields:

$$
\begin{aligned}
\omega_{\mu}^{a b}= & -2 e^{\nu[a} \partial_{[\mu} e_{\nu]}^{b]}-e^{\nu[a} e^{b] \sigma} e_{\mu c} \partial_{\sigma} e_{\nu}{ }^{c}-2 e_{\mu}{ }^{[a} e^{b] \nu} b_{\nu} \\
& -\frac{1}{4}\left(2 \bar{\psi}_{\mu}^{i} \gamma^{[a} \psi_{i}^{b]}+\bar{\psi}^{a i} \gamma_{\mu} \psi_{i}^{b}+\text { h.c. }\right), \\
\phi_{\mu}^{i}= & \left(\sigma^{\rho \sigma} \gamma_{\mu}-\frac{1}{3} \gamma_{\mu} \sigma^{\rho \sigma}\right)\left(\mathcal{D}_{\rho} \psi_{\sigma}^{i}-\frac{1}{8} \sigma^{a b} T_{a b}^{i j} \gamma_{\rho} \psi_{\sigma j}+\frac{1}{2} \sigma_{\rho \sigma} \chi^{i}\right), \\
f_{\mu}{ }^{a}= & \frac{1}{2} \widehat{R}_{\mu}{ }^{a}-\frac{1}{4}\left(D+\frac{1}{3} \widehat{R}\right) e_{\mu}^{a}-\frac{1}{2} i \widetilde{R}_{\mu a}(A)+\frac{1}{16} T_{\mu b}^{i j} T_{i j}^{a b},
\end{aligned}
$$

where

$$
\widehat{R}_{\mu}{ }^{a}=\left.\widehat{R}(M)_{\mu \nu}{ }^{a b} e_{b}{ }^{\nu}\right|_{f=0}
$$

is the Ricci tensor 7 constructed out of the curvature tensor $\widehat{R}(M)_{\mu \nu}^{a b}$ by contraction, but with the terms involving the conformal gauge field $f_{\mu}{ }^{a}$ omitted. $\widehat{R}=\widehat{R}_{\mu}{ }^{a} e_{a}{ }^{\mu}$ is the corresponding Ricci scalar. In a bosonic background and after Poincaré gauge fixing these expressions reduce to the standard Ricci tensor and Ricci scalar.

The independent gauge fields are the ones of general coordinate transformations $e_{\mu}{ }^{a}$, dilatations $b_{\mu}$, chiral $S U(2) \times U(1)$ transformations $\mathcal{V}_{\mu j}^{i}, A_{\mu}$ and $Q$-supertransformations $\psi_{\mu}^{i}$. The full content of the superconformal gauge multiplet is given by the independent gauge fields together with the auxiliary fields

$$
\left(e_{\mu}{ }^{a}, \psi_{\mu}^{i}, b_{\mu}, A_{\mu}, \mathcal{V}_{\mu}{ }^{i}{ }_{j}, T_{a b}^{i j}, \chi^{i}, D\right),
$$

so that there are $24+24$ off-shell degrees of freedom. The Weyl and chiral weights, and the chirality properties of the Weyl multiplet, of the dependent gauge fields and of the supersymmetry transformation parameters are listed in table 3.1.

One of the constraints is to set the curvature associated with local translations to zero, $R_{\mu \nu}(P)=0$ (3.22). This constraint enables one to interpret the translational gauge field $e_{\mu}{ }^{a}$ as a vielbein, because after imposing it this field transforms in the appropriate way under general coordinate transformations. The internal indices $a, b, \ldots$ can now be seen as tangent space indices and one can convert them into world indices using the vielbein. Note that the vielbein carries a non-trivial Weyl weight, so that the Weyl weight of a tensor changes when going from one type of indices to the other.

When solving the constraint one gets an expression for the Lorentz connection $\omega_{\mu}^{a b}$ in terms of the vielbein, the dilational gauge field $b_{\mu}$ and the $Q$-transformation gauge field $\psi_{\mu}{ }^{i}$ (3.30). Thus, $\omega_{\mu}^{a b}(e, b, \psi)$ is a superconformal generalization of the spin connection, in the sense that it does not only contain the standard terms that express the spin connection in terms of the vierbein, but also contains further terms involving the dilatational and $Q$-supertransformation gauge fields. Likewise, the modfied field strength $\widehat{R}_{\mu \nu}(M)^{a b}$ of Lorentz rotations is a superconformal version of the Riemann tensor. The expression (3.28) differs from the standard expression for the curvature tensor in terms of the spin connection,

$$
R_{\mu \nu}^{a b}=2 \partial_{[\mu} \omega_{\nu]}^{a b}-2 \omega_{[\mu}^{a b} \omega_{\nu]}^{c b} \eta_{b c},
$$

by additional terms involving the conformal gauge field $f_{\mu}{ }^{a}$ and the $Q$-gauge field $\psi_{\mu}{ }^{i}$. In the following we will refer to (3.34) as the Riemann tensor, because it reduces to the standard Riemann tensor when going to the super Poincaré theory by appropriate gauge fixing.

As already mentioned the Bianchi identities have to be modified after imposing the constraints. The modified Bianchi identity for the field strength $R_{\mu \nu}(P)$ is an algebraic equation which can be used to express the dilatational field strength in terms of the Lorentz field strength:

$$
\widehat{R}(M)_{[\mu \nu}^{a b} e_{\rho] b}=\widehat{R}_{[\mu \nu}(D) e_{\rho]}^{a} .
$$

\footnotetext{
${ }^{6}$ This tensor is not symmetric, when converting to pure world or pure tangent space indices.
} 
For the standard Riemann tensor the left hand side would be zero. Its non-vanishing reflects the presence of the dilatational gauge field $b_{\mu}$. Formula (3.35) is the non-standard Bianchi identity for the Riemann tensor in a theory with local scale invariance. Relation (3.35) leads to the pair-exchange property

$$
\widehat{R}(M)_{a b}^{c d}-\widehat{R}(M)^{c d}{ }_{a b}=2\left(\delta_{[a}^{[c} \widehat{R}(M)_{b]}^{d]}-\delta_{[a}^{[c} \widehat{R}(M)_{b]}^{d]}\right),
$$

where $\widehat{R}(M)_{\mu}^{a}=\widehat{R}(M)_{\mu \nu}{ }^{a b} e_{b}{ }^{\nu}$.

We now list some properties of the field strength that we will need later for constructing black hole solutions. It is convenient to define the modified field strength

$$
\mathcal{R}(M)_{a b}{ }^{c d}=\widehat{R}(M)_{a b}{ }^{c d}+\frac{1}{16}\left(T^{i j c d} T_{i j a b}+T_{a b}^{i j} T_{i j}^{c d}\right),
$$

where the $T^{2}$-modification cancels exactly the $T^{2}$-terms in the contribution to $\widehat{R}(M)$ from $f_{\mu}^{a}$. $\mathcal{R}(M)$ satisfies the following self conjugacy relations

$$
\begin{aligned}
\frac{1}{4} \varepsilon_{a b}{ }^{e f} \varepsilon^{c d}{ }_{g h} \mathcal{R}(M)_{e f}{ }^{g h} & =\mathcal{R}(M)_{a b}{ }^{c d} \\
\varepsilon_{a}{ }^{e c d} \mathcal{R}(M)_{c d b e} & =\varepsilon_{a}{ }^{e c d} \mathcal{R}(M)_{b e c d}=2 \widetilde{\widehat{R}}_{a b}(D)=2 i R_{a b}(A) .
\end{aligned}
$$

In the second line the relations (3.35) and (3.31) have been used.

It is also useful to introduce the following modified field strength for $S$-supertransformations:

$$
\mathcal{R}(S)_{a b}^{i}=R(S)_{a b}^{i}+\frac{3}{4} T_{a b}^{i j} \chi_{j} .
$$

The Bianchi identities and constraints imply that it satisfies

$$
\gamma^{a} \widetilde{\mathcal{R}}(S)_{a b}^{i}=2 D^{a} \widetilde{R}(Q)_{a b}^{i} .
$$

By contraction with $\gamma^{b} \gamma_{c d}$ one gets a relation between $\mathcal{R}(S)_{a b}^{i}$ and its dual:

$$
\mathcal{R}(S)_{a b}^{i}-\widetilde{\mathcal{R}}(S)_{a b}^{i}=2 \not D\left(R(Q)_{a b}^{i}+\frac{3}{4} \gamma_{a b} \chi^{i}\right) .
$$

Like the corresponding gauge field $\phi_{\mu}^{i}$, the field strengths $R(S)_{a b}^{i}$ and $\mathcal{R}(S)_{a b}^{i}$ have negative chirality.

Finally we note that the $Q$-field strength is related to the auxiliary field $\chi^{i}$ by

$$
\gamma^{a} R(Q)_{a b}^{i}+\frac{3}{2} \gamma_{b} \chi^{i}=0, \text { or } \chi^{i}=\frac{1}{6} \gamma^{a b} R(Q)_{a b}^{i} .
$$

Contraction with $\gamma^{b} \gamma_{e f}$ implies that $R(Q)_{a b}^{i}$ is antiselfdual. Like the corresponding gauge field $\psi_{\mu}^{i}$ the field strength $R(Q)_{a b}^{i}$ has positive chirality.

We now turn to the transformation properties of the fields under supersymmetry. As mentioned above $Q$-supertransformations have to close into general coordinate transformations, modulo other symmetries. The precise form in which successive $Q$-supertransformations act is the following:

$$
\left[\delta_{Q}\left(\epsilon_{1}\right), \delta_{Q}\left(\epsilon_{2}\right)\right]=\delta^{(\operatorname{cov})}(\xi)+\delta_{M}(\varepsilon)+\delta_{K}\left(\Lambda_{K}\right)+\delta_{S}(\eta)+\delta_{\text {gauge }} .
$$

Three comments are in order. The first is that $Q$-transformations do not close on a standard general coordinate transformation $\delta_{\text {gct }}(\xi)$ but involve a covariant general coordinate transformation, which is defined by

$$
\delta^{(\operatorname{cov})}(\xi)=\delta_{\mathrm{gct}}(\xi)+\sum_{T} \delta_{T}\left(-\xi^{\mu} h_{\mu}(T)\right) .
$$

The sum is over all superconformal transformations except the general coordinate transformation. If the field to which the above operator is applied transforms under additional gauge

\footnotetext{
${ }^{7}$ It is the integrability condition for expressing the spin connection in terms of the vielbein.
} 
symmetries, then these have to be included in the sum as well. The second comment is that there are additional Lorentz, special conformal and $S$-supertransformations on the right hand side of (3.43). The transformation parameters of these transformations and of the covariant general coordinate transformation are given in terms of the parameters $\left(\epsilon_{1}, \epsilon_{2}\right)$ of the two $Q$ supertransformations by

$$
\begin{aligned}
\xi^{\mu} & =2 \bar{\epsilon}_{2}^{i} \gamma^{\mu} \epsilon_{1 i}+\text { h.c. } \\
\varepsilon^{a b} & =\bar{\epsilon}_{1}^{i} \epsilon_{2}^{j} T_{i j}^{a b}+\text { h.c. } \\
\Lambda_{K}^{a} & =\bar{\epsilon}_{1}^{i} \epsilon_{2}^{j} D_{b} T_{i j}^{b a}-\frac{3}{2} \bar{\epsilon}_{2}^{i} \gamma^{a} \epsilon_{1 i} D+\text { h.c. } \\
\eta^{i} & =3 \bar{\epsilon}_{[1}^{i} \epsilon_{2]}^{j} \chi_{j} .
\end{aligned}
$$

The third remark is that whenever the field has additional gauge symmetries, like centralcharge gauge transformations or abelian or non-abelian gauge symmetries, then the gauge transformations $\delta_{\text {gauge }}$ are in general present on the right hand side. An example will be provided by the vector multiplets discussed in the next section.

For completeness we also list the commutators between $S$ and $Q$-supertransformations,

$$
\begin{aligned}
{\left[\delta_{S}(\eta), \delta_{Q}(\epsilon)\right]=} & \delta_{M}\left(2 \bar{\eta}^{i} \sigma^{a b} \epsilon_{i}+\text { h.c. }\right)+\delta_{D}\left(\bar{\eta}_{i} \epsilon^{i}+\text { h.c. }\right)+\delta_{A}\left(i \bar{\eta}_{i} \epsilon^{i}+\text { h.c. }\right) \\
& \left.+\delta_{V}\left(-2 \bar{\eta}^{i} \epsilon_{j}-\text { (h.c. } ; \text { traceless }\right)\right)
\end{aligned}
$$

and between two $S$-supertransformations,

$$
\left[\delta_{S}\left(\eta_{1}\right), \delta_{S}\left(\eta_{2}\right)\right]=\delta_{K}\left(\Lambda_{K}^{a}\right), \quad \text { with } \Lambda_{K}^{a}=\bar{\eta}_{2 i} \gamma^{a} \eta_{1}^{i}+\text { h.c. } .
$$

We conclude the section by presenting the transformation rules of the Weyl multiplet and of the dependent gauge fields under $Q-, S$ - and $K$-transformations. These relations are central for the construction of supersymmetric black hole solutions.

The transformation rules for the components of the Weyl multiplet are:

$$
\begin{aligned}
\delta e_{\mu}{ }^{a}= & \bar{\epsilon}^{i} \gamma^{a} \psi_{\mu i}+\text { h.c. } \\
\delta \psi_{\mu}^{i}= & 2 \mathcal{D}_{\mu} \epsilon^{i}-\frac{1}{8} \gamma_{a} \gamma_{b} T^{a b i j} \gamma_{\mu} \epsilon_{j}-\gamma_{\mu} \eta^{i}, \\
\delta b_{\mu}= & \frac{1}{2} \bar{\epsilon}^{i} \phi_{\mu i}-\frac{3}{4} \bar{\epsilon}^{i} \gamma_{\mu} \chi_{i}-\frac{1}{2} \bar{\eta}^{i} \psi_{\mu i}+\text { h.c. }+\Lambda_{K}^{a} e_{\mu}^{a}, \\
\delta A_{\mu}= & \frac{1}{2} i \bar{\epsilon}^{i} \phi_{\mu i}+\frac{3}{4} i \bar{\epsilon}^{i} \gamma_{\mu} \chi_{i}+\frac{1}{2} i \bar{\eta}^{i} \psi_{\mu i}+\text { h.c. }, \\
\delta \mathcal{V}_{\mu j}^{i}= & 2 \bar{\epsilon}_{j} \phi_{\mu}^{i}-3 \bar{\epsilon}_{j} \gamma_{\mu} \chi^{i}+2 \bar{\eta}_{j} \psi_{\mu}^{i}-\text { (h.c.; traceless), } \\
\delta T_{a b}^{i j}= & 8 \bar{\epsilon}^{i} \widehat{R}_{a b}(Q)^{j]} \\
\delta \chi^{i}= & -\frac{1}{12} \gamma_{a} \gamma_{b} D T^{a b i j} \epsilon_{j}+\frac{1}{6} \widehat{R}(V)^{i}{ }_{j \mu \nu} \gamma^{\mu} \gamma^{\nu} \epsilon^{j}-\frac{1}{3} i \widehat{R}(A)_{\mu \nu} \gamma^{\mu} \gamma^{\nu} \epsilon^{i} \\
& +D \epsilon^{i}+\frac{1}{12} T_{a b}^{i j} \gamma^{a} \gamma^{b} \eta_{j}, \\
\delta D= & \bar{\epsilon}^{i} D \chi_{i}+\text { h.c. },
\end{aligned}
$$

and the transformation rules of the dependent gauge fields are:

$$
\begin{aligned}
\delta \omega_{\mu}^{a b}= & -\bar{\epsilon}^{i} \sigma^{a b} \phi_{\mu i}-\frac{1}{2} \bar{\epsilon}^{i} T_{i j}^{a b} \psi_{\mu}^{j}+\frac{3}{2} \bar{\epsilon}^{i} \gamma_{\mu} \sigma^{a b} \chi_{i} \\
& +\bar{\epsilon}^{i} \gamma_{\mu} \widehat{R}^{a b}(Q)_{i}-\bar{\eta}^{i} \sigma^{a b} \psi_{\mu i}+\text { h.c. }+2 \Lambda_{K}^{[a} e_{\mu}^{b]}, \\
\delta \phi_{\mu}^{i}= & -2 f_{\mu}^{a} \gamma_{a} \epsilon^{i}-\frac{1}{4} D T_{c d}^{i j} \sigma^{c d} \gamma_{\mu} \epsilon_{j}+\frac{3}{2}\left[\left(\bar{\chi}_{j} \gamma^{a} \epsilon^{j}\right) \gamma_{a} \psi_{\mu}^{i}-\left(\bar{\chi}_{j} \gamma^{a} \psi_{\mu}^{j}\right) \gamma_{a} \epsilon^{i}\right] \\
& +\frac{1}{2} \widehat{R}(V)_{c d}{ }^{i}{ }_{j} \sigma^{c d} \gamma_{\mu} \epsilon^{j}+i \widehat{R}(A)_{c d} \sigma^{c d} \gamma_{\mu} \epsilon^{i}+2 \mathcal{D}_{\mu} \eta^{i}+\Lambda_{K}^{a} \gamma_{a} \psi_{\mu}^{i}, \\
\delta f_{\mu}^{a}= & -\frac{1}{2} \bar{\epsilon}^{i} \psi_{\mu}^{j} D_{b} T_{i j}^{b a}-\frac{3}{4} e_{\mu}{ }^{a} \bar{\epsilon}^{i} D \chi_{i}-\frac{3}{4} \bar{\epsilon}^{i} \gamma^{a} \psi_{\mu i} D \\
& +\bar{\epsilon}^{i} \gamma_{\mu} D_{b} \widehat{R}^{b a}(Q)_{i}+\frac{1}{2} \bar{\eta}^{i} \gamma^{a} \phi_{\mu i}+\text { h.c. }+\mathcal{D}_{\mu} \Lambda_{K}^{a} .
\end{aligned}
$$




\begin{tabular}{|c||cccccccc|ccc||cc|}
\hline & \multicolumn{10}{|c||}{ Weyl multiplet } & parameters \\
\hline \hline field & $e_{\mu}{ }^{a}{ }^{a}{ }_{\mu}^{i}$ & $b_{\mu}$ & $A_{\mu}$ & $\mathcal{V}_{\mu}{ }^{i}{ }_{j}$ & $T_{a b}^{i j}$ & $\chi^{i}$ & $D$ & $\omega_{\mu}^{a b}$ & $f_{\mu}{ }^{a}$ & $\phi_{\mu}^{i}$ & $\epsilon^{i}$ & $\eta^{i}$ \\
\hline \hline$w$ & -1 & $-\frac{1}{2}$ & 0 & 0 & 0 & 1 & $\frac{3}{2}$ & 2 & 0 & 1 & $\frac{1}{2}$ & $-\frac{1}{2}$ & $\frac{1}{2}$ \\
\hline$c$ & 0 & $-\frac{1}{2}$ & 0 & 0 & 0 & -1 & $-\frac{1}{2}$ & 0 & 0 & 0 & $-\frac{1}{2}$ & $-\frac{1}{2}$ & $-\frac{1}{2}$ \\
\hline$\gamma_{5}$ & & + & 1 & & & + & & & & - & + & - \\
\hline
\end{tabular}

Table 3.1: Weyl and chiral weights and fermion chirality ( $w$ and $c$ and $\gamma_{5}$, respectively) of the Weyl multiplet component fields and of the supersymmetry transformation parameters.

\begin{tabular}{|l|l|l|l|l|l|l|l|l|}
\hline Generator $T$ & $P^{a}$ & $M^{a b}$ & $D$ & $K^{a}$ & $Q^{i}$ & $S^{i}$ & $\left(V_{\Lambda}\right)^{i}{ }_{j}$ & $A$ \\
\hline Connection $h_{\mu}(T)$ & $e_{\mu}{ }^{a}$ & $\omega_{\mu}^{a b}$ & $b_{\mu}$ & $f_{\mu}{ }^{a}$ & $\frac{1}{2} \psi_{\mu}{ }^{i}$ & $\frac{1}{2} \phi_{\mu}{ }^{i}$ & $-\frac{1}{2} \mathcal{V}_{\mu}{ }^{i}{ }_{j}$ & $-i A_{\mu}$ \\
\hline Parameter & $\xi^{a}$ & $\varepsilon^{a b}$ & $\Lambda_{D}$ & $\Lambda_{K}^{a}$ & $\varepsilon^{i}$ & $\eta^{i}$ & $\Lambda_{V}{ }^{i}{ }_{j}$ & $\Lambda_{A}$ \\
\hline
\end{tabular}

Table 3.2: Table of superconformal gauge fields and transformation parameters

\subsubsection{Vector Multiplets}

We now turn to $\mathcal{N}=2$ vector multiplets $[103,104,105,106,82,16,90,92$, restricting ourselves to the case of abelian gauge symmetries. We consider $N_{V}+1$ vector multiplets, labeled by an index $I=0, \ldots, N_{V}$. One linear combination of the abelian gauge symmetries corresponds to the gauged central charge transformation, and the corresponding field strength belongs to the graviphoton. Note that we must have at least one vector multiplet in the theory in order to make contact with $\mathcal{N}=2$ Poincaré supergravity, because the Weyl multiplet does not account for the graviphoton.

A conformal $\mathcal{N}=2$ vector multiplet

$$
\mathbf{X}^{I}=\left(X^{I}, \Omega_{i}^{I}, W_{\mu}^{I}, Y_{i j}^{I}\right)
$$

has $8+8$ off-shell degrees of freedom and consists of a complex scalar $X^{I}$, an $S U(2)$ doublet of chiral fermions $\Omega_{i}^{I}$, called gaugini, a vector gauge field $W_{\mu}^{I}$ and a real $S U(2)$ triplet of auxiliary scalars $Y_{i j}^{I}$ (this means $Y_{i j}^{I}=Y_{j i}^{I}$ and $Y_{i j}^{I}=\varepsilon_{i k} \varepsilon_{j l} Y^{k l I}$ ). The Weyl and chiral weights and the chirality properties of the component fields are listed in table 3.3. The superconformally covariant field strength is defined by

$$
\mathcal{F}_{\mu \nu}^{I}=F_{\mu \nu}^{I}-\left(\varepsilon_{i j} \bar{\psi}_{[\mu}^{i} \gamma_{\nu]} \Omega^{j I}+\varepsilon_{i j} \bar{X}^{I} \bar{\psi}_{\mu}^{i} \psi_{\nu}^{j}+\frac{1}{4} \varepsilon_{i j} \bar{X}^{I} T_{\mu \nu}^{i j}+\text { h.c. }\right),
$$

where

$$
F_{\mu \nu}^{I}=2 \partial_{[\mu} W_{\nu]}^{I}
$$

is the standard abelian field strength. The covariant field strength satisfies the Bianchi identity

$$
D^{b}\left(\mathcal{F}_{a b}^{+I}-\mathcal{F}_{a b}^{-I}+\frac{1}{4} X^{I} T_{a b i j} \varepsilon^{i j}-\frac{1}{4} \bar{X}^{I} T_{a b}^{i j} \varepsilon_{i j}\right)=\frac{3}{4}\left(\bar{\chi}^{i} \gamma_{a} \Omega^{I j} \varepsilon_{i j}-\bar{\chi}_{i} \gamma_{a} \Omega_{j}^{I} \varepsilon^{i j}\right) .
$$

The selfdual and antiselfdual part $\mathcal{F}_{a b}^{ \pm I}$ of $\mathcal{F}_{a b}^{I}$ are defined according to the conventions explained in appendix A. The components of the multiplet transform as follows under supersymmetry:

$$
\begin{aligned}
\delta X^{I} & =\bar{\epsilon}^{i} \Omega_{i}^{I}, \\
\delta \Omega_{i}^{I} & =2 \not D X^{I} \epsilon_{i}+\frac{1}{2} \varepsilon_{i j} \mathcal{F}^{I \mu \nu-} \gamma_{\mu} \gamma_{\nu} \epsilon^{j}+Y_{i j}^{I} \epsilon^{j}+2 X^{I} \eta_{i}, \\
\delta W_{\mu}^{I} & =\varepsilon^{i j} \bar{\epsilon}_{i} \gamma_{\mu} \Omega_{j}^{I}+2 \varepsilon_{i j} \bar{\epsilon}^{i} \bar{X}^{I} \psi_{\mu}^{j}+\text { h.c. } \\
\delta Y_{i j}^{I} & =2 \bar{\epsilon}_{(i} \not D \Omega_{j)}^{I}+2 \varepsilon_{i k} \varepsilon_{j l} \bar{\epsilon}^{k} \not D \Omega^{l) I} .
\end{aligned}
$$




\begin{tabular}{|c||cccc|}
\hline \multicolumn{1}{|c||}{} & \multicolumn{4}{c|}{ vector multiplet } \\
\hline \hline field & $X^{I}$ & $\Omega_{i}^{I}$ & $W_{\mu}^{I}$ & $Y_{i j}^{I}$ \\
\hline \hline $\mathrm{w}$ & 1 & $\frac{3}{2}$ & 0 & 2 \\
\hline $\mathrm{c}$ & -1 & $-\frac{1}{2}$ & 0 & 0 \\
\hline$\gamma_{5}$ & \multicolumn{4}{c}{+} \\
\hline
\end{tabular}

Table 3.3: Weyl and chiral weights and fermion chirality ( $w$ and $c$ and $\gamma_{5}$, respectively) of the vector multiplet component fields

These transformation rules satisfy relation (3.43), including a field-dependent gauge transformation on the right-hand side, which acts with the following parameter:

$$
\theta^{I}=4 \varepsilon^{i j} \bar{\epsilon}_{2 i} \epsilon_{1 j} X^{I}+\text { h.c. } .
$$

The covariant field strength transforms as follows under supersymmetry,

$$
\delta \mathcal{F}_{a b}^{I}=-2 \varepsilon^{i j} \bar{\epsilon}_{i} \gamma_{[a} D_{b]} \Omega_{j}^{I}-2 \varepsilon^{i j} \bar{\eta}_{i} \sigma_{a b} \Omega_{j}^{I}+\text { h.c. . }
$$

\subsubsection{Chiral Multiplets}

The gauge invariant quantities of the Weyl multiplet and of vector multiplets sit in chiral multiplets [107, 104, 108, 108, 102, 109, 16, 90, 92], which therefore are the building blocks of the action. We will now discuss these multiplets.

A (left-handed) $\mathcal{N}=2$ chiral multiplet $\widehat{\mathbf{A}}$ is obtained from a general scalar $\mathcal{N}=2$ superfield by imposing the chirality constraint

$$
\mathbf{D}^{i} \widehat{\mathbf{A}}=0,
$$

where

$$
\mathbf{D}^{i}=\frac{\partial}{\partial \bar{\theta}^{i}}+\gamma^{\mu} \theta^{i} \frac{\partial}{\partial x^{\mu}}
$$

is the right-handed superderivative in $\mathcal{N}=2$ superspace with coordinates $\left(x^{\mu}, \theta^{i}\right)$. A chiral multiplet has $16+16$ off-shell components,

$$
\widehat{\mathbf{A}}=\left(\widehat{A}, \widehat{\Psi}_{i}, \widehat{B}_{i j}, \widehat{F}_{a b}^{-}, \widehat{\Lambda}_{i}, \widehat{C}\right),
$$

namely two complex scalars $\widehat{A}, \widehat{C}$, a complex $S U(2)$-triplet of scalars $\widehat{B}_{i j}$, an antiselfdual Lorentz tensor $\widehat{F}_{a b}^{-}$and two $S U(2)$ doublets of left-handed fermions $\widehat{\Psi}_{i}, \widehat{\Lambda}_{i}$. The Lagrangian also contains the conjugated right-handed chiral multiplet. When coupling to conformal supergravity one has to assign Weyl and chiral weights $(w, c)$ to a chiral superfield. The weights of the component fields are fixed by the ones of the lowest component, $(w, c)$ as indicated in table 3.4. A consistent coupling to conformal supergravity in addition requires $w=-c$.

In the special case $w=-c=1$ one can impose the further constraint 102

$$
\left(\varepsilon_{i j} \overline{\mathbf{D}}^{i} \sigma_{a b} \mathbf{D}^{j}\right)^{2}(\widehat{\mathbf{A}})^{\star}=\mp 96 \square \widehat{\mathbf{A}},
$$

which reduces the number of independent off-shell components to $8+8$. The resulting multiplet is called the restricted chiral multiplet. In the case of rigid $\mathcal{N}=2$ supersymmetry the constraint allows one to express $\widehat{C}$ and $\widehat{\Lambda}_{i}$ in terms $\widehat{A}$ and $\widehat{\Psi}_{i}$, respectively, and it imposes a

\footnotetext{
${ }^{8}$ We put a hat on the chiral superfield and on its components in order to have the same notation as in the following sections and chapters, where a background chiral superfield will play an important role.
} 
reality constraint on $\widehat{B}_{i j}$ and the standard Bianchi identity of an abelian field strength on $\widehat{F}_{a b}^{-}$. When coupling to $\mathcal{N}=2$ conformal supergravity the equations are more complicated due to additional terms needed for covariantization. The independent components $\widehat{A}, \widehat{\Psi}_{i}, \widehat{F}_{a b}^{-}, \widehat{B}_{i j}$ of a reduced chiral multiplet can both in the rigid and in the local case be identified with the convariant quantities $X^{I}, \Omega_{i}^{I}, \mathcal{F}_{a b}^{-I}, Y_{i j}^{I}$ associated with a vector multiplet. In particular $\widehat{F}_{a b}^{-}$can be interpreted as a field strength, because it satisfies the appropriate Bianchi identity (which, in the local case, is (3.53).)

The covariant quantities of the Weyl multiplet are associated with a reduced chiral multiplet $\mathbf{W}_{a b}^{i j}$ 110.9 This multiplet is obtained by imposing the constraints 3.57,3.60 on a $\mathcal{N}=2$ superfield which has an antiselfdual tensor field $\widehat{A}_{a b}^{i j}$ as its lowest component, with weights $w=-c=1$. The higher components are a left-handed antisymmetric tensor spinor $\widehat{\Psi}_{a b}^{i}$, a triplet of tensor fields $\widehat{B}_{a b}{ }^{i}$, a tensor $\widehat{F}_{a b}{ }^{c d}$, which is antisymmetric and antiselfdual in both pairs of indices, a left-handed tensor-spinor $\widehat{\chi}_{a b i}$ and a tensor field $\widehat{C}_{a b i j}$. The lowest component of $\mathbf{W}_{a b}^{i j}$ is the auxiliary $T$-field, $\widehat{A}_{a b}^{i j}=T_{a b}^{i j}$, whereas the higher components are related to the $Q$-field strength $\widehat{R}(Q)_{a b}^{i}$, the $S U(2)$-field strength $\widehat{R}(V)_{a b}{ }_{j}{ }_{j}$, the modified Lorentz field strength $\mathcal{R}(M)_{a b}{ }^{c d}$, the modified $S$-field strength $\mathcal{R}(S)_{a b i}$ and to auxiliary fields. We will not give the explicit relations here, because the superconformal field strengths will finally enter the action in terms of yet another chiral multiplet, that we will discuss next. But note that all field strengths associated with independent gauge fields appear in $\mathbf{W}_{a b}^{i j}$.

Chiral multiplets can be multiplied, and the product is another chiral multiplet. Weyl and chiral weights behave additive in products. Therefore the product of two reduced chiral multiplets is a non-reduced chiral multiplet 102]. The multiplet which contains the superconformal field strength in the action is a non-reduced chiral multiplet $\mathbf{W}^{2}=(\widehat{A}, \ldots)$ of weights $w=-c=2$, which is obtained by contracting the superfield $\mathbf{W}_{a b}^{i j}$ with itself in the following way:

$$
\mathbf{W}^{2}=\varepsilon_{i k} \varepsilon_{j l} \mathbf{W}_{a b}^{i j} \mathbf{W}^{a b k l} .
$$

By a long and tedious calculation the components of this multiplet are found to be the following 110, 91]:

$$
\begin{aligned}
\widehat{A}= & \left(\varepsilon_{i j} T_{a b}^{i j}\right)^{2} \\
\widehat{\Psi}_{i}= & 16 \varepsilon_{i j} R(Q)_{a b}^{j} T^{k l a b} \varepsilon_{k l}, \\
\widehat{B}_{i j}= & -16 \varepsilon_{k(i} R(V)^{k}{ }_{j) a b} T^{l m a b} \varepsilon_{l m}-64 \varepsilon_{i k} \varepsilon_{j l} \bar{R}(Q)_{a b}^{k} R(Q)^{l a b}, \\
\widehat{F}^{-a b}= & -16 \mathcal{R}(M)_{c d}^{a b} T^{k l c d} \varepsilon_{k l}-16 \varepsilon_{i j} \bar{R}(Q)_{c d}^{i} \gamma^{a b} R(Q)^{j c d}, \\
\widehat{\Lambda}_{i}= & 32 \varepsilon_{i j} \gamma_{a b} R(Q)_{c d}^{j} \mathcal{R}(M)_{c d}^{a b}+16\left(\mathcal{R}(S)_{a b i}+3 \gamma_{[a} \mathcal{D}_{b]} \chi_{i}\right) T^{k l a b} \varepsilon_{k l} \\
& -64 R(V)_{a b i}^{k} \varepsilon_{k l} R(Q)_{a b}^{l} \\
\widehat{C}= & 64 \mathcal{R}(M)_{c d}^{-a b} \mathcal{R}(M)_{c d}^{-a b}+32 R(V)_{a b l}^{-k} R(V)_{a b k}^{-l} \\
& -32 T^{i j a b} D_{a} D^{c} T_{c b i j}+128 \overline{\mathcal{R}}(S)_{i}^{a b} R(Q)_{a b}^{i}+384 \bar{R}(Q)^{a b i} \gamma_{a} D_{b} \chi_{i}
\end{aligned}
$$

The highest component $\widehat{C}$ contains terms quadratic and linear in the curvature and plays a central role in the $\mathcal{N}=2$ Lagrangian and in the computation of black hole entropy. We will see that the matching between macroscopic and microscopic black hole entropy depends on the precise value of the coefficients in $\widehat{C}$.

\subsubsection{The Non-linear Multiplet}

We have to introduce one further multiplet, which will be used later to consistently gauge-fix the superconformal theory to a super Poincaré theory. This multiplet was introduced for precisely this purpose in 15]. It is called the non-linear multiplet, because some of its components

\footnotetext{
${ }^{9} \mathbf{W}_{a b}^{i j}$ denotes the full $\mathcal{N}=2$ superfield.
} 


\begin{tabular}{|c||c|c|c|c|c|c|}
\hline Field & $\widehat{A}$ & $\widehat{\Psi}_{i}$ & $\widehat{B}_{i j}$ & $\widehat{F}_{a b}^{-}$ & $\widehat{\Lambda}_{i}$ & $\widehat{C}$ \\
\hline \hline Weyl weight & $w$ & $w+\frac{1}{2}$ & $w+1$ & $w+1$ & $w+\frac{3}{2}$ & $w+2$ \\
\hline Chiral weight & $c$ & $c+\frac{1}{2}$ & $c+1$ & $c+1$ & $c+\frac{3}{2}$ & $c+2$ \\
\hline Chirality & & + & & & + & \\
\hline
\end{tabular}

Table 3.4: Weyl and chiral weights and chiralities of the components of a chiral multiplet

\begin{tabular}{|c||c|c|c|c|}
\hline Field & $\Phi_{\alpha}^{i}$ & $\lambda^{i}$ & $M^{i j}$ & $V_{a}$ \\
\hline \hline Weyl weight & 0 & $\frac{1}{2}$ & 1 & 1 \\
\hline Chiral weight & 0 & $-\frac{1}{2}$ & -1 & 0 \\
\hline Chirality & & - & & \\
\hline
\end{tabular}

Table 3.5: Weyl weights, chiral weights and chirality of the components of the non-linear multiplet

transform into products of other components. For all other multiplets considered before the nonlinear terms in the transformation rules are entirely due to superconformal covariantizations.

A non-linear multiplet has the following components:

$$
\left(\Phi_{\alpha}^{i}, \lambda^{i}, M^{i j}, V_{a}\right)
$$

The field $\Phi=\Phi_{\alpha}^{i}$ is an $S U(2)$ matrix of scalar fields. Note that it has, besides the $S U(2)$ index $i$ a second index $\alpha=1,2$. This index is associated with an additional rigid $S U(2)$ symmetry, which acts from the right, whereas the local $S U(2)$, which is part of the superconformal group, acts from the left. The hermitean conjugate is denoted $\Phi^{+}=\Phi^{\alpha}{ }_{i}$. Since the matrix is an element of $S U(2)$, we have the constraints

$$
\Phi \Phi^{+}=\mathbb{I}=\Phi^{+} \Phi \text { and } \operatorname{det} \Phi=1 .
$$

Therefore $\Phi$ describes 3 real scalars. The scalars have weigth zero. The other components are a spinor doublet $\lambda^{i}$, a complex antisymmetric matrix $M^{i j}$ of Lorentz scalars and a real Lorentz vector $V_{a}$. The weights and chiral properties are listed in table 3.5.

Naive counting yields $9+8$ degrees of freedom. This indicates the presence of a constraint. When constructing the transformation rules

$$
\begin{aligned}
\delta \Phi_{\alpha}^{i}= & \left(2 \bar{\epsilon}^{i} \lambda_{j}-\delta_{j}^{i} \bar{\epsilon}^{k} \lambda_{k}-\text { h.c. }\right) \Phi_{\alpha}^{j} \\
\delta \lambda^{i}= & -\frac{1}{2} \gamma^{a} V_{a} \epsilon^{i}-\frac{1}{2} M^{i j} \epsilon_{j}-2 \lambda^{i}\left(\bar{\lambda}^{j} \epsilon_{j}+\bar{\lambda}_{j} \epsilon^{j}\right)+\gamma^{a} \epsilon^{i}\left(\bar{\lambda}^{j} \gamma_{a} \lambda_{j}\right), \\
& -2 \sigma_{a b} \epsilon_{j} \bar{\lambda}^{j} \sigma^{a b} \lambda^{i}+\Phi^{i}{ }_{\alpha} \gamma^{a} D_{a} \Phi^{\alpha}{ }_{j} \epsilon^{j}+\eta^{i}, \\
\delta M^{i j}= & 6 \bar{\epsilon}^{[i} \chi^{j]}+\bar{\epsilon}^{k} \sigma^{a b} T_{a b}^{-i j} \lambda_{k}-2 \bar{\epsilon}^{[i} \gamma^{a} V_{a} \lambda^{j]}-2 \bar{\epsilon}^{k} \lambda_{k} M^{i j}, \\
& +4 \bar{\epsilon}^{[i}\left(\gamma^{a} D_{a} \lambda^{j]}+\Phi^{j]}{ }_{\alpha} \gamma^{a} D_{a} \Phi^{\alpha}{ }_{k} \lambda^{k}\right) \\
\delta V_{a}= & {\left[\frac{3}{2} \bar{\epsilon}^{i} \gamma_{a} \chi_{i}-\frac{1}{4} \bar{\epsilon}^{i} \gamma_{a} \sigma_{b c} T_{i j}^{+b c} \lambda^{j}-\bar{\epsilon}^{i} \gamma_{a} \gamma^{b} V_{b} \lambda_{i}+\bar{\epsilon}^{i} \gamma_{a} \lambda^{j} M_{i j},\right.} \\
& \left.+4 \bar{\epsilon}^{i} \sigma_{a b} D^{b} \lambda_{i}+2 \bar{\epsilon}_{i} \gamma_{a} \Phi^{i}{ }_{\alpha} \gamma^{b} D_{b} \Phi^{\alpha}{ }_{j} \lambda^{j}-\bar{\lambda}_{i} \gamma_{a} \eta^{i}+\text { h.c. }\right]+2 \Lambda_{K a},
\end{aligned}
$$

one finds that one needs to impose the supersymmetric constraint

$$
D^{a} V_{a}-3 D-\frac{1}{2} V^{a} V_{a}-\frac{1}{4}\left|M_{i j}\right|^{2}+D^{a} \Phi_{\alpha}^{i} D_{a} \Phi_{i}^{\alpha}+\text { fermions }=0
$$


in order to close the algebra. This is interpreted as a constraint on the vector $V_{a}$ which reduces the degrees of freedom to $8+8$. When considering the coupling to the minimal $\mathcal{N}=2$ representation, we will give a somewhat different interpretation.

A particular property of the vector $V_{a}$ is that it transforms under special conformal transformations,

$$
\delta_{K} V_{a}=2 \Lambda_{K a} .
$$

The other components transform trivially, like almost all other independent fields in the other superconformal multiplets. As a consequence the covariant derivative of $V_{a}$ contains the Ktransformation gauge field:

$$
D^{a} V_{a}=\mathcal{D}^{a} V_{a}-2 f_{a}^{a}+\text { fermionic terms . }
$$

This will be used later.

\subsection{Superconformal Actions}

In the preceeding sections we outlined the construction of various off-shell representations of the $\mathcal{N}=2$ superconformal algebra. The next step is to find the action. We will first explain the basic ideas then describe how to find the action for $N_{V}+1$ abelian vector multiplets coupled to conformal supergravity.

An elementary method for constructing invariant actions is the well known Noether method. One first writes down all the terms that one wants to have in the Lagrangian. Then one iteratively adds terms to compensate for the non-invariance of the terms already present until one has found an invariant.

Since the method is tedious, especially for theories with a large number of degrees of freedom, additional methods are helpfull. In the context of rigid supersymmetry, for instance, one is used to the fact that the highest component of a chiral superfield transforms into a total derivative, $\delta_{Q}(\epsilon) C=\partial_{\mu}(\cdots)$. Therefore $\int d^{4} x C$ is an invariant and a candidate for an invariant action, though it is of course not guaranteed to be a physically sensitive choice. When coupling to $\mathcal{N}=2$ conformal supergravity things are more complicated: There are additional terms present in the transformation rules and one wants to have an invariant with respect to all superconformal transformations. Using the Noether method one can find the necessary covariantizations. The result is a so called density formula, which specifies a quantity that transforms into a total deriviative under all superconformal transformations. In the case of a chiral multiplet the density formula takes the form 102], 92]:

$$
\begin{aligned}
e^{-1} \mathcal{L}= & C-\varepsilon^{i j} \bar{\psi}_{i}^{\mu} \gamma_{\mu} \Lambda_{j}-\frac{1}{4} \bar{\psi}_{\mu i} \sigma_{a b} T_{j k}^{a b} \gamma^{\mu} \psi_{l} \varepsilon^{i j} \varepsilon^{k l} \\
& -\frac{1}{16} A\left(T_{a b i j} \varepsilon^{i j}\right)^{2}-\bar{\psi}_{\mu i} \sigma^{\mu \nu} \psi_{\nu i} B_{k l} \varepsilon^{i k} \varepsilon^{j l} \\
& \bar{\psi}_{\mu i} \psi_{\nu j} \varepsilon^{i j}\left(F^{-\mu \nu}-\frac{1}{2} A T_{k l}^{\mu \nu} \varepsilon^{k l}\right) \\
& -\frac{1}{2} \varepsilon^{i j} \varepsilon^{k l} e^{-1} \varepsilon^{\mu \nu \rho \sigma} \bar{\psi}_{\mu i} \psi_{\nu j}\left(\bar{\psi}_{\rho k} \gamma_{\sigma} \psi_{l}+\bar{\psi}_{\rho k} \psi_{\sigma l} A\right)+\text { h.c. }
\end{aligned}
$$

Note that the weight of the chiral multiplet is not arbitrary. The action $S=\int d^{4} x \mathcal{L}$ has to be Weyl and chirally invariant. Since the integration measure has weights $w=-4, c=0$ it follows that the $C$ must have $w_{C}=4, c_{C}=0$. This means that the lowest component has weights $w_{A}=-c_{A}=2$. As a consequence one needs a weight 2 chiral superfield to define the Lagrangian.

Similar density formulae can be derived for other multiplets. In order to put them to use one needs another ingredient. In practice one knows a density formula, like the one for a chiral multiplet, and wants to use it to construct an action for one or several other multiplets,

\footnotetext{
${ }^{10}$ In the construction of the action we need to distinguish two chiral multiplets by notation. The chiral multiplet appearing in the density formula and a background chiral multiplet which encodes the higher curvature terms. They are denoted by $(A, \ldots, C)$ and $(\widehat{A}, \ldots, \widehat{C})$, respectively.
} 
like a collection of vector multiplets. Then one needs to know how to construct a (weight 2) chiral multiplet (or another multiplet for which a density formula is known) out of the vector multiplets (or out of other whatever multiplets are to appear in the action). The techniques that enable one to get one multiplet out of another are known as the multiplet calculus, and in our particular context as the $\mathcal{N}=2$ superconformal multiplet calculus [16].

We will now outline the construction of an action for several abelian vector multiplets using the chiral density formula. The most general choice for the lowest component of the chiral multiplet in the density formula is a function of the vector multiplet scalars $X^{I}, I=0, \ldots, N_{V}$ 102,16 :

$$
A \sim F\left(X^{I}\right) .
$$

The function $F\left(X^{I}\right)$, which is called the prepotential, is subject to two restrictions: First, it needs to be holomorphic in the sense that it does not depend on the complex conjugated scalars $\bar{X}^{I}$. The second restriction, which does not apply to the case of rigid supersymmetry, follows from the fact that $A$ must have weight $2(w=-c=2)$ in the presence of conformal supergravity. Since a vector multiplet has weight $1(X$ has $w=-c=1)$ this implies that the function $F\left(X^{I}\right)$ must be homogenous of degree 2 :

$$
F\left(\lambda X^{I}\right)=\lambda^{2} F\left(X^{I}\right)
$$

for all $\lambda \in \mathbb{C}-\{0\}$.

The chiral density and the action depend on the Weyl multiplet only through the superconformal covariantizations. When gauge-fixing the theory to obtain $\mathcal{N}=2$ Poincaré supergravity coupled to $N_{V}$ vector multiplets way as in the toy example discussed earlier and there are no higher powers of the Riemann tensor in the action. The theory also contains minimal terms for the other fields, only, where minimal means terms with up to two derivatives and up to four fermions.

In order to have higher curvature terms in the Lagrangian one has to include explicit couplings to the Weyl multiplet. Since the covariant quantities of the Weyl multiplet sit in the chiral multiplet $\mathbf{W}^{2}$, only this multiplet can appear in the Lagrangian. For simplicity we will call the $\mathbf{W}^{2}$-multiplet the Weyl multiplet in the following. It will be clear from the context whether we refer to the multiplet of superconformal gauge fields or to the corresponding chiral multiplet. The problem of finding the coupling of vector multiplets to the Weyl multiplet is the same as finding the coupling to a background chiral multiplet, because the only relevant fact for constructing the coupling is the type of multiplet we want to couple to. Since the $R^{2}$-Lagrangian is very complicated and non-linear it is useful to work with a chiral background field as long as possible and to plug in the explicit expressions for the Weyl multiplet only at the end. The coupling to the background field is described by a function $F$, which now also depends on the lowest component of the chiral multiplet $\widehat{\mathbf{A}}=(\widehat{A}, \ldots)$ [111. The function must be holomorphic and homogenous of second degree in both $X^{I}$ and $\widehat{A}$ :

$$
F\left(\lambda X^{I}, \lambda^{w} \widehat{A}\right)=\lambda^{2} F\left(X^{I}, \widehat{A}\right),
$$

where $w$ is the weight of the chiral background field. ${ }^{12}$

The homogenity of $F\left(X^{I}, \widehat{A}\right)$ implies several useful identities between the function and its derivatives. We use the following notation for derivatives:

$$
F_{I}:=\frac{\partial}{\partial X^{I}} F\left(X^{I}, \widehat{A}\right), \quad F_{\widehat{A}}:=\frac{\partial}{\partial \widehat{A}} F\left(X^{I}, \widehat{A}\right),
$$

and consequently for higher deriviatives:

$$
F_{I_{1} \cdots I_{k} \widehat{A} \cdots \widehat{A}}=\frac{\partial}{\partial X^{I_{1}}} \cdots \frac{\partial}{\partial X^{I_{k}}} \frac{\partial}{\partial \widehat{A}} \cdots \frac{\partial}{\partial \widehat{A}} F\left(X^{I}, \widehat{A}\right) .
$$

\footnotetext{
${ }^{11}$ Actually this requires to add one further compensating multiplet. We will come back to this later.

12 For $w \neq 1$ this is a slightly generalized definition of 'homogenous', which allows the variable $\widehat{A}$ to be 'weighted'.
} 
Then, by differentiating the defining relation (3.72) with respect to $\lambda$, and setting $\lambda=1$, we get

$$
X^{I} F_{I}+w \widehat{A} F_{\widehat{A}}=2 F\left(X^{I}, \widehat{A}\right) .
$$

This is an alternative definition of a homogenous function (of degree 2), the so-called Euler relation. Further identities are found by taking derivatives with respect to $X^{I}$ or $\widehat{A}$, for example

$$
X^{I} F_{I J}+w \widehat{A} F_{J \widehat{A}}=F_{J}
$$

When assuming that the function $F\left(X^{I}, \widehat{A}\right)$ has a power expansion around $\widehat{A}=0$ one defines a family of functions $F^{(g)}\left(X^{I}\right)$ by

$$
F\left(X^{I}, \widehat{A}\right)=\sum_{g=0}^{\infty} F^{(g)}\left(X^{I}\right) \widehat{A}^{g} .
$$

Note that the $F^{(g)}$ are homogenous of degree $2-g w$ in $X^{I}$. When the background field is taken to be the Weyl multiplet ( $\mathbf{W}^{2}$ multiplet), then $w=2$. The first function $F^{(0)}\left(X^{I}\right)$ in the expansion is the prepotential, and controls the minimal terms in the action.

The full $\mathcal{N}=2$ superconformally invariant action for $N_{V}+1$ vector multiplets coupled to conformal supergravity is obtained by using the chiral density formula (3.69) for a chiral multiplet with lowest component $A=-\frac{i}{2} F\left(X^{I}, \widehat{A}\right)$. Since we will be interested in black holes, which are purely bosonic solutions to the field equations, we will only need to display the bosonic part, which reads 111]:

$$
\begin{aligned}
e^{-1} \mathcal{L} \sim & {\left[i \bar{F}_{I} X^{I}\left(\frac{1}{6} R-D\right)+i \mathcal{D}_{\mu} F_{I} \mathcal{D}^{\mu} \bar{X}^{I}\right.} \\
& +\frac{1}{4} i F_{I J}\left(F_{a b}^{-I}-\frac{1}{4} \bar{X}^{I} T_{a b}^{i j} \varepsilon_{i j}\right)\left(F_{a b}^{-J}-\frac{1}{4} \bar{X}^{J} T_{a b}^{i j} \varepsilon_{i j}\right)-\frac{1}{8} i F_{I}\left(F_{a b}^{+I}-\frac{1}{4} X^{I} T_{a b i j} \varepsilon^{i j}\right) T_{i j}^{a b} \varepsilon^{i j} \\
& -\frac{1}{8} i F_{I J} Y_{i j}^{I} Y^{J i j}-\frac{i}{32} F\left(T_{a b i j} \varepsilon^{i j}\right)^{2} \\
& +\frac{1}{2} i F_{\widehat{A}} \widehat{C}-\frac{1}{8} i F_{\widehat{A} \widehat{A}}\left(\varepsilon^{i k} \varepsilon^{j l} \widehat{B}_{i j} \widehat{B}_{k l}-2 \widehat{F}_{a b}^{-} \widehat{F}_{a b}^{-}\right)+\frac{1}{2} i \widehat{F}_{a b}^{-} F_{\widehat{A} I}\left(F_{a b}^{-I}-\frac{1}{4} \bar{X}^{I} T_{a b}^{i j} \varepsilon_{i j}\right) \\
& \left.-\frac{1}{4} i \widehat{B}_{i j} F_{\widehat{A} I} Y^{I i j}\right]+ \text { h.c. }
\end{aligned}
$$

Let us comment on the various terms of this lenghty expression. The two terms in the first line result from decomposing the term $i \bar{F}_{I} \square_{\mathrm{sc}} X^{I}+$ h.c., where $\square_{\mathrm{sc}}$ is the superconformal d'Alembertian, $\square_{\mathrm{sc}}=D^{a} D_{a}$, in terms of expressions which are natural from the super Poincaré perspective. One piece can be written in terms of the bosonic covariant derivative $\mathcal{D}_{\mu}$ and after a partial integration one gets the second term in the first line. The rest contains the auxiliary $D$ field plus a curvature piece which modulo fermionic pieces is proportional to the Ricci scalar associated with the spin connection $\omega_{\mu}^{a b}$. As in the toy example the special conformal gauge field has dropped out. Note however that we are still in a conformally invariant theory and therefore the spin connection still contains the dilatational gauge field. These considerations explain the first term in the first line, which has a piece that should become the Einstein-Hilbert term when going to the super Poincare theory. It is accompanied by an awkward looking term linear in the auxiliary field $D$. We will have to deal with this term later on.

In the following lines we get terms involving the gauge fields. Note that we have rewritten the superconformally covariant field strengths $\mathcal{F}_{a b}^{I}$ in terms of the standard field strength $F_{a b}^{I}$ in order to make explicit the dependence on the auxiliary field $T_{a b}^{i j}$. The terms in the last two lines explicitly involve the chiral background field. The most important term is $\frac{1}{2} i F_{\widehat{A}} \widehat{C}+$ h.c. which, according to (3.62), contains curvature squared terms. We will have a closer look at these terms later. The minimally coupled case is obtained by setting the chiral background to zero. Then, the last two lines are absent and the function $F\left(X^{I}, \widehat{A}\right)$ reduces to the prepotential $F\left(X^{I}\right)$. 


\subsection{Symplectic Reparametrizations}

As is well known, in extended supergravity models the full set of field equations (including the Bianchi identities) is invariant under continuous transformations which nowadays are called duality transformations 112. These transformations generalize the electric-magnetic duality transformations of Maxwell electrodynamics and like them they are - in general - not symmetries, because they are not invariances of the action and act non-trivially on the couplings. In supergravity theories couplings are scalar field dependent, and therefore supersymmetry implies that the scalars must transform as well. A particular structure occurs in the case of $\mathcal{N}=2$ vector multiplets, where gauge fields and scalars sit in the same supermultiplet. In this case the duality transformations manifest themselves as symplectic reparametrizations as we will review in this section [83]. We will follow the work [111] of de Wit, who analysed symplectic reparametrizations in a chiral background.

Since duality transformations generalize electric-magnetic duality, they can only exist if the action depends on the field strength, but not explicitly depends on the gauge potential. Therefore they are restricted to the case of abelian vector multiplets. This is precisely the case we are interested in.

The action that we found in the last section contains terms quadratic and linear in the field strength $F_{\mu \nu}^{I-}$. It is convenient to introduce a so-called dual field strength 13 by

$$
G_{I}^{-\mu \nu}=\frac{2 i}{e} \frac{\partial \mathcal{L}}{\partial F_{\mu \nu}^{I-}}
$$

so that the Lagrangian takes the form

$$
e^{-1} \mathcal{L} \sim-\frac{i}{2}\left(F_{\mu \nu}^{-I} G_{I}^{-\mu \nu}-\text { h.c. }\right)+\cdots .
$$

For the superconformal action whose bosonic part was displayed in the last section the dual gauge field takes the form

$$
G_{\mu \nu I}^{-}=F_{I J} F_{\mu \nu}^{-J}+\mathcal{O}_{\mu \nu I}^{-},
$$

where $\mathcal{O}_{\mu \nu I}^{-}$are all the terms that couple linearly to the field strength. When restricting ourselves to the bosonic fields this term takes the form

$$
\mathcal{O}_{\mu \nu I}^{-}=\frac{1}{4}\left(\bar{F}_{I}-F_{I J} \bar{X}^{J}\right) T_{\mu \nu}^{i j} \varepsilon_{i j}+\widehat{F}_{\mu \nu}^{-} F_{I \widehat{A}} .
$$

In the full theory this term also contains fermions. Clearly fermions have to transform under duality too, as they sit in the same supermultiplet as the gauge fields. But since we are interested in bosonic field configurations, only, we will restrict our discussion of duality to the bosonic fields. We also note that the second derivatives $F_{I J}$ of the function $F\left(X^{I}, \widehat{A}\right)$ obviously encode the field dependent couplings and $\theta$ angles. T4

The field equations and Bianchi identities take the form垉

$$
\begin{aligned}
\mathcal{D}_{\mu}\left(G_{I}^{-\mu \nu}-G_{I}^{+\mu \nu}\right) & =0 \\
\mathcal{D}_{\mu}\left(F^{-I \mu \nu}-F^{+I \mu \nu}\right) & =0 .
\end{aligned}
$$

The combined set of equations is manifestly invariant under the duality rotation

$$
\left(\begin{array}{c}
F_{\mu \nu}^{ \pm I} \\
G_{J \mu \nu}^{ \pm}
\end{array}\right) \rightarrow\left(\begin{array}{cc}
U_{K}^{I} & Z^{I L} \\
W_{J K} & V_{J}{ }^{L}
\end{array}\right)\left(\begin{array}{l}
F_{\mu \nu}^{ \pm K} \\
G_{L \mu \nu}^{ \pm}
\end{array}\right)=\left(\begin{array}{l}
\breve{F}_{\mu \nu}^{ \pm I} \\
\breve{G}_{J \mu \nu}^{ \pm}
\end{array}\right),
$$

\footnotetext{
${ }^{13}$ Note that this is in general not the Hodge dual. See appendix $\mathrm{B}$ for more details.

${ }^{14}$ This term gets modified when eliminating the auxiliary fields. Then $F_{I J}$ is replaced by another quantity usually denoted $\overline{\mathcal{N}}_{H}$. We will come back to this later.

${ }^{15}$ See appendix $\mathrm{B}$ for more details.
} 
if the matrix

$$
\mathcal{O}=\left(\begin{array}{cc}
U^{I}{ }_{K} & Z^{I L} \\
W_{J K} & V_{J}{ }^{L}
\end{array}\right)
$$

is real and invertible, $\mathcal{O} \in \mathrm{GL}\left(2 N_{V}+2, \mathbb{R}\right)$. In fact the choice of $\mathcal{O}$ is even more restricted, because we want to relate the transformed set of equations to a dual Lagrangian

$$
e^{-1} \breve{\mathcal{L}} \sim-\frac{i}{2}\left(\breve{F}_{\mu \nu}^{-I} \breve{G}_{I}^{-\mu \nu}-\text { h.c. }\right) .
$$

This Lagrangian must again have the structure that we found in the last section and therefore

$$
\breve{G}_{\mu \nu I}^{-}=\breve{F}_{I J} \breve{F}_{\mu \nu}^{-J}+\breve{\mathcal{O}}_{\mu \nu I}^{-} \text {. }
$$

This implies that $F_{I J}$ has to transform as

$$
\breve{F}_{I J}=[V F+W]_{I L}\left[(U+Z F)^{-1}\right]_{J}^{L},
$$

i.e. by a projective linear transformation and $\mathcal{O}_{\mu \nu}^{-I}$ must transform as

$$
\breve{\mathcal{O}}_{\mu \nu I}^{-}=\mathcal{O}_{\mu \nu J}^{-}\left[(U+Z F)^{-1}\right]_{I}^{J} .
$$

Inside such matrix equations we use the notation $F=\left(F_{I J}\right)$. The symmetric matrix $F_{I J}$ has to be mapped to a symmetric matrix $\breve{F}_{I J}$. This implies that (up to a uniform scale transformation, that we neglect) the matrix $\mathcal{O}$ has to be symplectic, $\mathcal{O} \in \operatorname{Sp}\left(2 N_{V}+2, \mathbb{R}\right)$ :

$$
\mathcal{O}^{T} \Omega \mathcal{O}=\Omega, \text { where } \Omega=\left(\begin{array}{cc}
0 & \mathbb{I} \\
-\mathbb{I} & 0
\end{array}\right) .
$$

In terms of the block matrices this means

$$
U^{T} W-W^{T} U=0=Z^{T} V-V^{T} Z \text { and } U^{T} V-W^{T} Z=\mathbb{I} .
$$

We have now to remind ourselves that $F_{I J}$ are the second derivatives of the function $F\left(X^{I}, \widehat{A}\right)$. Since $F_{I J}$ transforms, the function itself has to transform in such a way that the correct transformation of $F_{I J}$ follows. As a consequence various terms in the action besides the ones discussed so far transform under duality as well. This is no surprise, since the field strengths sit in the same $\mathcal{N}=2$ multiplet as the scalars. What we have to find is a transformation rule for the $X^{I}$ that precisely induces the correct transformation rule of $F_{I J}$. The additional chiral background field $\widehat{A}$ sits in a different supermultiplet and therefore is inert under duality rotations. Note however that it will enter into the transformation rules because of its appearence in $F\left(X^{I}, \widehat{A}\right)$.

In order to find the transformation rule of the scalars it is convenient first to focus on the expression $\left(X^{I}, F_{J}\right)^{T}$ and to postpone making the connection to the function $F\left(X^{I}, \widehat{A}\right)$. The reason is that the correct transformation law for $F_{I J}$ is induced by simply requiring that $\left(X^{I}, F_{J}\right)$ transforms linearly under symplectic transformations:

$$
\left(\begin{array}{c}
X^{I} \\
F_{J}
\end{array}\right) \longrightarrow\left(\begin{array}{cc}
U_{K}^{I} & Z^{I L} \\
W_{J K} & V_{J}^{L}
\end{array}\right)\left(\begin{array}{c}
X^{K} \\
F_{L}
\end{array}\right)=\left(\begin{array}{c}
\breve{X}^{I} \\
\breve{F}_{J}
\end{array}\right) .
$$

Computing $\breve{F}_{I J}=\frac{\partial}{\partial \check{X}^{J}} \breve{F}_{I}$ one indeed finds (3.88). Quantities transforming linearly under the symplectic group are called symplectic vectors. Given two symplectic vectors $v, w$ one can form a symplectic scalar by taking the symplectic scalar product, $-i \bar{v}^{T} \Omega w$. The symplectic scalar product of $\left(X^{I}, F_{J}\right)$ with itself, $-i\left(F_{I} \bar{X}^{I}-X^{I} \bar{F}_{I}\right)$ enters the action as the coefficient of the Ricci scalar. Thus the invariance of this term is manifest. Moreover this is another way of seeing why one cannot allow more general duality rotations than symplectic ones. 
The next step is to take into account that $X^{I}$ and $F_{J}$ are not independent quantities. $F_{J}$ is a function of the $X^{I}$ (and of the chiral background) and therefore all transformations have to be induced by transformations of the scalars $X^{I}$. From (3.92) we can read off that the new scalars are given by the field-dependent transformation

$$
\breve{X}^{I}=U^{I} X^{J}+Z^{I K} F_{K}=\left(U^{I}{ }_{J}+Z^{I K} F_{K J}\right) X^{J}+w \widehat{A} Z^{I K} F_{K \widehat{A}} .
$$

This transformation has to be invertible, ${ }^{16}$ because the number of independent scalar fields should not change under a duality transformation. Finally one can find the new function $\breve{F}\left(\breve{X}^{I}, \widehat{A}\right)$ by integrating 3.92$)$ :

$$
\begin{aligned}
\breve{F}(\breve{X}, \widehat{A})= & F(X, \widehat{A})-\frac{1}{2} X^{I} F_{I}+\frac{1}{2}\left(U^{T} W\right)_{I J} X^{I} X^{J}+\frac{1}{2}\left(U^{T} V+W^{T} Z\right)_{I}{ }^{K} X^{I} F_{K} \\
& +\frac{1}{2}\left(Z^{T} V\right)^{I J} F_{I} F_{J} .
\end{aligned}
$$

The integration constant is fixed by the homogenity properties of the function. Note that $F(X, \widehat{A})$ does not transform as a function under symplectic transformations $F(X, \widehat{A}) \neq$ $\breve{F}(\breve{X}, \widehat{A})$. An example of an expression transforming as a function is

$$
F(X, \widehat{A})-\frac{1}{2} X^{I} F_{I}=\breve{F}(\breve{X}, \widehat{A})-\frac{1}{2} \breve{X}^{I} \breve{F}_{I} .
$$

Such objects, which turn out to be rare, are called symplectic functions. Note that being a symplectic function refers to a simple, tensoriel transformation behaviour, which is not to be confused with an invariance property. An expression is called invariant (with respect to one, several or all symplectic transformations) if the functional dependence on the variables is not changed under the transformation,

$$
\breve{f}(\breve{X}, \widehat{A})=f(\breve{X}, \widehat{A}) .
$$

If the function $F(X, \widehat{A})$ is invariant under a duality transformation, then this transformation is a symmetry of the theory. Note that this is possible even though $F(X, \widehat{A})$ is not a symplectic function. The $T$-duality symmetries of string theory, which in $\mathcal{N}=2$ compactifications of heterotic string theories are realized as a subset of the symplectic reparametrizations, are examples of such duality symmetries.

In order to write down the transformation rules for some of the derivatives of $F(X, \widehat{A})$ it is convenient to introduce the following definitions:

$$
\begin{aligned}
\mathcal{S}(X, \widehat{A})^{I}{ }_{J} & :=\frac{\partial \breve{X}^{I}}{\partial X^{J}}=U_{J}^{I}+Z^{I K} F_{K J} \\
\mathcal{Z}^{I J} & :=\left[\mathcal{S}^{-1}\right]_{K}^{I} Z^{K J} \\
N_{I J} & :=2 \operatorname{Im} F_{I J} \\
N^{I J} & :=\left[N^{-1}\right]^{I J}
\end{aligned}
$$

For the lowest derivatives of $F(X, \widehat{A})$ one finds the following rules:

$$
\begin{aligned}
\breve{N}_{I J} & =N_{K L}\left[\overline{\mathcal{S}}^{-1}\right]_{I}^{K}\left[\mathcal{S}^{-1}\right]_{J}^{L} \\
\breve{N}^{I J} & =N^{K L} \overline{\mathcal{S}}_{K}^{I}{ }_{K} \mathcal{S}_{L}^{J} \\
\breve{F}_{I J K} & =F_{M N P}\left[\mathcal{S}^{-1}\right]_{I}^{M}\left[\mathcal{S}^{-1}\right]_{J}^{N_{J}}\left[\mathcal{S}^{-1}\right]_{K}^{P}
\end{aligned}
$$

\footnotetext{
${ }^{16}$ This changes when we go to the super Poincaré theory, because the gauge fixing imposes one relation among the scalars. We will come back to this later.
} 
and

$$
\begin{aligned}
\breve{F}_{\widehat{A}} & =F_{\widehat{A}}, \\
\breve{F}_{\widehat{A} I} & =F_{\widehat{A} J}\left[\mathcal{S}^{-1}\right]_{I}^{J}, \\
\breve{F}_{I}-\breve{F}_{I J} \breve{X}^{J} & =\left[F_{J}-F_{J K} X^{K}\right]\left[\mathcal{S}^{-1}\right]_{I}^{J}, \\
\breve{F}_{I}-\breve{F}_{I J} \breve{X}^{J} & =\left[F_{J}-\bar{F}_{J K} X^{K}\right]\left[\overline{\mathcal{S}}^{-1}\right]^{J}{ }_{I}, \\
\breve{F}_{\widehat{A} \widehat{A}} & =F_{\widehat{A} \widehat{A}}-F_{\widehat{A} I} F_{\widehat{A} J} \mathcal{Z}^{I J}
\end{aligned}
$$

and so on. Note that $F_{\widehat{A}}$ is a symplectic function, whereas all other derivatives of $F(X, \widehat{A})$ are not. The above formulae are sufficient to verify that the terms $\mathcal{O}_{I \mu \nu}^{-}$(3.82) transform as required by (3.89).

Though it can be shown that the field equations are invariant under continuous $S p\left(2 N_{V}+\right.$ $2, \mathbb{R})$ transformations, one expects that this group is broken to a discrete subgroup, denoted by $S p\left(2 N_{V}+2, \mathbb{Z}\right)$ at the non-perturbative level. One way of seeing this is to make the connection with electric-magnetic duality transformations more explicit. To do so one writes the vector kinetic term as

$$
e^{-1} \mathcal{L}=\frac{1}{8} N_{I J} F_{\mu \nu}^{I} F_{\mu \nu}^{J}+\frac{i}{4} \Theta_{I J} F_{\mu \nu}^{I} \widetilde{F}^{\mu \nu J},
$$

where $N_{I J}$ was defined above and $\Theta_{I J}=\operatorname{Re} F_{I J}$. Obviously, $N_{I J}$ encodes the gauge couplings, whereas $\Theta_{I J}$ are the $\Theta$-angles. The symplectic transformations contain a subgroup of the form $U=V=\mathbb{I}, Z=\mathbb{O}$ and $W_{I J}=\Delta \Theta_{I J}$, which acts by constant shifts of the $\Theta$-angles. Perturbatively such shifts can be ignored but non-perturbatively this is not guaranteed. If, for instance, the $U(1)$ effective field theory considered here comes from a spontanously broken $S U(2)$ Yang-Mills theory, then there will be instantons, and this restricts the $\Theta$-shifts to a discrete subgroup.

Another way of seeing the reduction from a continuous to a discrete group is to take into account the presence of electric and magnetic charges. If the effective $U(1)$ theory is obtained by integrating out the massive degrees of freedom of a string theory, then the theory contains electrically and magnetically charged massive states. Part of them are elementary string states, wheras the rest is realized as solitons. String dualities require that at the non-perturbative level both electric and magnetic charges with respect to all $U(1)$ factors exist. Thus, at the nonperturbative level one has to take into account that the theory contains electric and magnetic charges. According to the generalized Dirac quantization rule [113, 114, 115, 116] the allowed values of electric and magnetic charges are discrete and form a lattice.

The magnetic and electric charges $\left(p^{I}, q_{J}\right)$ are the sources for the gauge fields $\left(F_{\mu \nu}^{I}, G_{\mu \nu J}\right)$. They are defined by

$$
\begin{aligned}
& p^{I}=\frac{1}{4 \pi} \oint F^{I}=\frac{1}{4} \int_{0}^{2 \pi} \int_{0}^{\pi} F_{23}^{I} r^{2} \sin \theta d \theta d \phi \\
& q_{J}=\frac{1}{4 \pi} \oint G_{I}=\frac{1}{4} \int_{0}^{2 \pi} \int_{0}^{\pi} G_{J 23} r^{2} \sin \theta d \theta d \phi
\end{aligned}
$$

where $F_{a b}^{I}, G_{J a b}, a, b=0,1,2,3$ are the tangent space components of the field strength. The definition of charges is such that gauge fields with the asymptotic behaviour

$$
F_{23}^{I} \simeq_{r \rightarrow \infty} \frac{p^{I}}{r^{2}}, \quad G_{I 23} \simeq_{r \rightarrow \infty} \frac{q_{I}}{r^{2}},
$$

carry charges $\left(p^{I}, q_{I}\right)$. Note that $\left(p^{I}, q_{J}\right)$ transforms as a vector under symplectic $S p\left(2 N_{V}+\right.$ $2, \mathbb{R})$ transformations. A general continous symplectic transformation will not map the charge lattice onto itself but will deform it into a different lattice. This means that the spectrum of admissible charged states is not invariant. If such states are actually present, as it happens 
in string theory, then one has to restrict the symplectic transformations to a discrete subgoup $S p\left(2 N_{V}+2, \mathbb{Z}\right)$, which by definition maps the charge lattice to itself. When normalizing the gauge fields appropriately, this is just the subgroup of matrices with integer entries. Again we have reached the conclusion that the symplectic group is reduced to a discrete subgroup. Since duality rotations are not automatically symmetries of one given Lagrangian, this discrete group is the maximal possible group of duality symmetries. In string compactifications one can have, depending on the amount of unbroken supersymmetry various discrete duality symmetries, which are called T-, S- and U-duality. These are always proper subgroups of the discrete symplectic group.

\subsection{Poincaré Supergravity}

\subsubsection{Poincaré Gauge Fixing}

Our motivation for constructing $\mathcal{N}=2$ conformal supergravity is not to study it as a theory in its own right but to use it as a tool for dealing with $\mathcal{N}=2$ Poincaré supergravity. We now will discuss how to go from the superconformal to the super Poincaré theory by imposing gauge conditions, following [15], 92]. More generally, we would like to know how to go back and forth between the two formulations, because this gives us the option of analysing questions about the Poincaré theory in the conformal set-up. Since the Poincaré theory is a gauge fixed version of the conformal theory, what we have to do is to find appropriate gauge conditions and to identify gauge invariant quantities. The usage of the gauge invariant, conformal formulation is sometimes advantageous because here more symmetries are realized in a simple linear way, quantities transform in a simple and systematic way and the off-shell multiplets are smaller. In the gauge-fixed Poincaré theory symmetries and in particular supersymmetry are realized in a more complicated non-linear way. Moreover the multiplets are larger and since the graviphoton now belongs to the gravity supermultiplet instead of sitting in a separate vector multiplet, symplectic reparametrizations are more complicated.

We first sketch the gauge fixing procedure using the most simple example, the construction of pure $\mathcal{N}=2$ supergravity (without higher derivative terms). Then we give a more detailed account for the case where an arbitrary number of abelian vector multiplets is present together with a chiral background describing higher derivative terms.

The standard $\mathcal{N}=2$ Poincaré supergravity multiplet has $40+40$ off-shell degrees of freedom 85. 86, 82. 17 The physical degrees of freedom are the graviton $e_{\mu}{ }^{a}$, two gravitini $\psi_{\mu}^{i}$ and one gauge field, the graviphoton. On the superconformal side we have to use the Weyl multiplet, which has $24+24$ off-shell degrees of freedom, to describe the graviton and the gravitini. Since this does not account for the graviphoton, the natural thing is to add one vector multiplet $\left(X, F_{\mu \nu}, \ldots\right)$. The resulting reducible representation with $32+32$ degrees of freedom is called the $\mathcal{N}=2$ minimal field representation, because it is mandatory if one wants to describe $\mathcal{N}=2$ Poincaré supergravity. The coupling to the Weyl multiplet is described by a prepotential, which has to be holomorphic and homogenous of degree 2 . If only one vector multiplet is present, then up to normalization the unique choice therefore is $F(X)=X^{2}$. The vector multiplet contains a scalar $X$, which cannot be a physcial degree of freedom in the Poincaré theory. But from the toy example discussed earlier we expect that dilatational gauge fixing will be done by setting this scalar to a constant. This is indeed the case, but we will not enter into the details here, because we will discuss this in a more general situation later. At this point we have accounted for all physical degrees of freedom of the Poincaré theory. But nevertheless it is clear that this cannot be the full story.

The most obvious indication is that the minimal field representation has $8+8$ degrees of freedom less then the $40+40$ of the off-shell super Poincaré multiplet. A closer inspection shows that these additional degrees of freedom are indeed needed to accomplish the Poincaré gauge fixing, because the fields in the minimal field representation cannot be used as compensators

\footnotetext{
${ }^{17} \mathrm{As}$ mentioned at the beginning of the chapter there exist smaller off-shell representations. Within the superconformal approach we only know how to make contact with the $40+40$ multiplet.
} 
for the chiral $S U(2)$ symmetry. A related point, that we already mentioned, is that the action for the minimal field representation (plus $N_{V} \geq 0$ additional vector multiplets) has an awkward term linear in the auxiliary $D$-field, that leads to inconsistent equations of motions unless further terms are added.

Thus one always has to add further superconformal matter multiplets to the minimal field representation in order to describe Poincaré supergravity. There are three known choices for a second compensator multiplet with $8+8$ degrees of freedom: One can use a so-called non-linear multiplet, a hypermultiplet or a tensor multiplet.

We will now go through the gauge fixing procedure in some more detail and consider the more general case, where one starts with the Weyl multiplet and $\left(N_{V}+1\right)$ abelian vector multiplets. In this case one linear combination of the field strengths provides the graviphoton whereas the corresponding scalar becomes dependent on the others through a gauge condition. This way one arrives at $\mathcal{N}=2$ supergravity coupled to $N_{V}$ vector multiplets. The couplings are encoded in the function $F\left(X^{I}, \widehat{A}\right)$, where the chiral background field $\widehat{\mathbf{A}}$ is identified with the Weyl multiplet $\mathbf{W}^{2}$.

The first step is to break special conformal invariance by imposing the $K$-gauge $b_{\mu}=0$. Since this constraint is not invariant under the remaining transformations, one has to modify the rules for $Q$ - and $S$-supertransformations and for dilatations by a compensating, field dependent $K$ transformation. Note, however, that this does not modify the transformation properties of the other components of the Weyl multiplet and of the components of the vector multiplet, because these are $K$-independent. Only $b_{\mu}$ and the composite gauge fields transform non-trivially under special conformal transformations.

The second step is to gauge-fix dilatations. From the toy example we expect that fixing the dilatations will eliminate one scalar field and will lead to a standard Einstein-Hilbert term with a constant coefficient instead of a field dependent one. Therefore the natural choice is the $D$-gauge

$$
-i\left(X^{I} \bar{F}_{I}-F_{I} \bar{X}^{I}\right)=1\left(=m_{\text {Planck }}^{2}\right) .
$$

This condition is manifestly symplectic. Moreover it is dimensionful as expected for a constraint that breaks scale invariance. The constant on the right hand side should therefore be related to the natural scale of Poincaré gravity, the Planck mass. We will postpone fixing the overall normalization of our Lagrangian, because there will be another contribution to the Einstein-Hilbert term. Except where dimensional analysis is required we will use Planckian units, $m_{\text {Planck }}=1$. One can now proceed by fixing the chiral $U(1)$ symmetry, for example by imposing the $A$-gauge

$$
X^{0}=\bar{X}^{0} .
$$

Both the $D$ - and the $A$-gauge are constraints on the scalars $X^{I}$. As a consequence the Poincaré theory has only $N_{V}$ independent scalars. Further analysis leads to the notion of special Kähler geometry, that we will discuss in 3.5.3. At this point two comments are in order: First, for many purposes it is useful not to fix the gauge but to stay in the superconformal setup and to work with appropriate gauge invariant variables under dilatations and $U(1)$ transformations. Second, the above gauge choices are not unique and depending on the problem under considerations different choices might be useful.

In order to break $S$-supersymmetry one imposes another constraint, called the $S$-gauge. This constraint can be solved by eliminating one of the vector multiplet fermions. As a result the physical fields now precisely correspond to the $\mathcal{N}=2$ Poincaré gravity multiplet plus $N_{V}$ vector multiplets. To be precise the $S$-gauge also breaks $Q$-supersymmetry, but a combination of a $Q$-transformation and a compensating $S$-transformation is preserved. Furthermore one has to combine this with a compensating $K$-transformation which restores the $K$-gauge. Therefore the Poincaré supertransformations take the form

$$
\delta_{Q}^{\text {Poincaré }}(\epsilon)=\delta_{Q}(\epsilon)+\delta_{S}(\eta)+\delta_{K}\left(\Lambda_{K}\right),
$$

with suitable field dependent choinces of $\eta$ and $\Lambda_{K}$. The above formula is an example of a decomposition rule, that is a rule which displays a combination of symmetries that is left 
unbroken by a gauge choice. Obviously supersymmetry is realized in a much more complicated way in the Poincaré theory than in the conformal theory.

Finally we have to discuss the gauge-fixing of chiral $S U(2)$ transformations. We already mentioned that one has to add another multiplet in order to provide the necessary compensators. For definiteness we will use the non-linear multiplet. The correct coupling can be found by the following reasoning: As already mentioned the Lagrangian of the minimal field representation (with $N_{V}$ vector multiplets added) has a term linear in the auxiliary $D$ field,

$$
e^{-1} \mathcal{L} \sim-i\left(X^{I} \bar{F}_{I}-F_{I} \bar{X}^{I}\right)\left(D-\frac{1}{6} R\right)+\cdots
$$

which leads to inconsistent equations of motion. We have displayed the Einstein-Hilbert term as well for reasons that will become obvious in a second. The idea is to couple the non-linear multiplet such that all linear terms involving $D$ are precisely canceled. We now remind ourselves that the non-linear multiplet is subject to the constraint

$$
D^{a} V_{a}-3 D-\frac{1}{2} V^{a} V_{a}-\frac{1}{4}\left|M_{i j}\right|^{2}+D^{a} \Phi_{\alpha}^{i} D_{a} \Phi_{i}^{\alpha}+\cdots=0,
$$

where we neglected the fermionic terms, because we are only inerested in the bosonic Lagrangian. The constraint can be interpreted as a constraint on the vector $V_{a}$, but now we take it as the defining equation of $D$ in terms of the non-linear multiplet and take $V_{a}$ to be unconstrained. When coupling the non-linear multiplet to the $\mathcal{N}=2$ minimal field representation this has the net effect of adding $8+8$ degrees of freedom, so that one has a total of $40+40$ (not counting the additional $N_{V}$ vector multiplets). Since the constraint contains $D$ linearly one takes the Lagrangian of the non-linear multiplet to be proportional to the constraint. The normalization of this term is chosen such that the terms involving $D$ cancel in the full Lagrangian. At this point one has to keep in mind that $V_{a}$ transforms under $K$-transformations. As a consequence its covariant derivative contains a term linear in $D$ :

$$
D^{a} V_{a}=\mathcal{D}^{a} V_{a}-2 f_{a}^{a}+\cdots=\mathcal{D}^{a} V_{a}+2 D-\frac{1}{3} R+\cdots
$$

and the term to be added to the Lagrangian is proportional to

$$
\mathcal{D}^{a} V_{a}-D-\frac{1}{3} R-\frac{1}{2} V^{a} V_{a}-\frac{1}{4}\left|M_{i j}\right|^{2}+D^{a} \Phi_{\alpha}^{i} D_{a} \Phi_{i}^{\alpha}+\text { fermions } .
$$

Comparing this to (3.106) we see that we have to add the term

$$
e^{-1} \Delta \mathcal{L}=-i\left(X^{I} \bar{F}_{I}-F_{I} \bar{X}^{I}\right)\left(\mathcal{D}^{a} V_{a}-\frac{1}{3} R-D+\cdots\right)
$$

on the right hand side. The resulting bosonic Lagrangian is

$$
\begin{aligned}
8 \pi e^{-1} \mathcal{L}= & \left(-i\left(X^{I} \bar{F}_{I}-F_{I} \bar{X}^{I}\right)\right) \cdot\left(-\frac{1}{2} R\right) \\
& +\left[i \mathcal{D}_{\mu} F_{I} \mathcal{D}^{\mu} \bar{X}^{I}\right. \\
& +\frac{1}{4} i F_{I J}\left(F_{a b}^{-I}-\frac{1}{4} \bar{X}^{I} T_{a b}^{i j} \varepsilon_{i j}\right)\left(F_{a b}^{-J}-\frac{1}{4} \bar{X}^{J} T_{a b}^{i j} \varepsilon_{i j}\right)-\frac{1}{8} i F_{I}\left(F_{a b}^{+I}-\frac{1}{4} X^{I} T_{a b i j} \varepsilon^{i j}\right) T_{i j}^{a b} \varepsilon^{i j} \\
& -\frac{1}{8} i F_{I J} Y_{i j}^{I} Y^{J i j}-\frac{i}{32} F\left(T_{a b i j} \varepsilon^{i j}\right)^{2} \\
& +\frac{1}{2} i F_{\widehat{A}} \widehat{C}_{-}-\frac{1}{8} i F_{\widehat{A} \widehat{A}}\left(\varepsilon^{i k} \varepsilon^{j l} \widehat{B}_{i j} \widehat{B}_{k l}-2 \widehat{F}_{a b}^{-} \widehat{F}_{a b}^{-}\right)+\frac{1}{2} i \widehat{F}_{a b}^{-} F_{\widehat{A} I}\left(F_{a b}^{-I}-\frac{1}{4} \bar{X}^{I} T_{a b}^{i j} \varepsilon_{i j}\right) \\
& \left.-\frac{1}{4} i \widehat{B}_{i j} F_{\widehat{A} I} Y^{I i j}+\text { h.c. }\right] \\
& -i\left(X^{I} \bar{F}_{I}-F_{I} \bar{X}^{I}\right) \cdot\left(\mathcal{D}^{a} V_{a}-\frac{1}{2} V^{a} V_{a}-\frac{1}{4}\left|M_{i j}\right|^{2}+D^{a} \Phi_{\alpha}^{i} D_{a} \Phi^{\alpha}{ }_{i}\right) .
\end{aligned}
$$

Note that we now have fixed the overall normalization of our Lagrangian. We have chosen it such that when imposing the $D$-gauge $-i\left(X^{I} \bar{F}_{I}-F_{I} \bar{X}^{I}\right)=m_{\text {Planck }}^{2}$ the Einstein-Hilbert term takes the form

$$
e^{-1} \mathcal{L}_{E H}=-\frac{1}{2 \kappa^{2}} R
$$


where $\kappa$ is the gravitational coupling, which is related to Newtons constant by $\kappa^{2}=8 \pi G_{N}$. In natural units $(\hbar=c=1)$ we have $G_{N}=l_{\text {Planck }}^{2}=m_{\text {Planck }}^{-2}$ so that

$$
e^{-1} \mathcal{L}_{E H}=-\frac{m_{\text {Planck }}^{2}}{16 \pi} R
$$

which explains the factor $8 \pi$ on the left hand side of (3.111).

After discussing the Einstein-Hilbert term we next display the curvature squared terms in the Lagrangian. To do so we need to work out the term

$$
8 \pi e^{-1} \mathcal{L}=\frac{i}{2} F_{\widehat{A}} \widehat{C}+\text { h.c. }+\cdots .
$$

First recall that the highest component $\widehat{C}$ of the $\mathbf{W}^{2}$ superfield is given by

$$
\widehat{C}=64 \mathcal{R}(M)_{c d}^{-a b} \mathcal{R}(M)_{a b}^{-} c d+\cdots,
$$

where the neglected terms are at most linear in the curvature. Next recall that

$$
\mathcal{R}(M)_{a b}{ }^{c d}=R_{a b}{ }^{c d}-4 \delta_{[a}^{[c} f_{b]}^{d]}+\cdots,
$$

where the omitted terms do not depend on the curvature. But the $K$-gauge field $f_{a}{ }^{b}$ is a composite field which depends on the curvature. Substituting the explicit form we find

$$
\mathcal{R}(M)_{a b}{ }^{c d}=C_{a b}{ }^{c d}+\cdots,
$$

where $C_{a b}{ }^{c d}$ reduces to the Weyl tensor in the Poincaré frame. As a consequence we have

$$
\widehat{C}=64 C_{c d}^{-{ }^{a b}} C_{a b}^{-c d}+\cdots=: 64\left(C_{a b c d}^{-}\right)^{2}+\cdots .
$$

This motivates the name Weyl multiplet.

Next the function $F_{\widehat{A}}$ is the derivative of $F(X, \widehat{A})$ with respect to $\widehat{A}=T_{a b}^{-} T^{-a b}=:\left(T_{a b}^{-}\right)^{2}$, where $T_{a b}^{-}$is the auxiliary $T$-field. Thus

$$
F_{\widehat{A}}=F^{(1)}(X)+2 F^{(2)}(X)\left(T_{a b}^{-}\right)^{2}+\cdots .
$$

Therefore we get a series of interaction terms which are quadratic in the Weyl tensor and even powers in the $T$-field, with field dependent couplings $F^{(g \geq 1)}$ :

$$
8 \pi e^{-1} \mathcal{L}=32 i\left(F^{(1)}(X)\left(C^{-}\right)^{2}+2 F^{(2)}(X)\left(C^{-}\right)^{2}\left(T^{-}\right)^{2}+3 F^{(3)}(X)\left(C^{-}\right)^{2}\left(T^{-}\right)^{4}+\cdots\right)+\text { h.c. }
$$

For $g=1$ we get purely gravitational couplings, which can be rewritten as

$$
8 \pi e^{-1} \mathcal{L}=-32 \operatorname{Im} F^{(1)}(X)\left(C_{a b d c}\right)^{2}-32 i \operatorname{Re} F^{(1)}(X) C_{a b c d} \widetilde{C}^{a b c d} .
$$

The first term is the Weyl action with a field dependent coupling. The second term is real (in Minkowski signature) in view of our definition of the dual tensor, which includes a factor $i$. It is a 'gravitational $\theta$-term', because $C_{a b c d} \widetilde{C}^{a b c d}=R_{a b c d} \widetilde{R}^{a b c d}$ and $R_{a b c d} \widetilde{R}^{a b c d}$ is proportional to the Hirzebruch signature density. We already remarked in chapter 2 that this action resembles a Yang-Mills action in many respects.

We also note that our Lagrangian contains various other higher derivative terms. For example when expanding the 'gauge kinetic term' we find a series of higher powers of the auxiliary $T$-field:

$$
8 \pi e^{-1} \mathcal{L}=\frac{i}{4} F_{a b}^{I-} F^{J-a b}\left(F_{I J}^{(0)}(X)+F_{I J}^{(1)}(X)\left(T_{a b}^{-}\right)^{2}+F_{I J}^{(2)}(X)\left(T_{a b}^{-}\right)^{4}+\cdots\right)+\text { h.c. }
$$

Finally we comment on the terms in the last line of (3.111), which come from the second compensating multiplet. We note that when imposing the $D$-gauge the term $\mathcal{D}^{a} V_{a}$ becomes a total derivative and can be dropped. The local chiral $S U(2)$ invariance can be gauge-fixed by imposing the $V$-gauge

$$
\Phi_{\alpha}^{i}=\delta_{\alpha}^{i} .
$$

Once this is imposed the theory is only invariant under rigid $S U(2)_{R}$ rotations, which are automorphisms of the super Poincaré algebra.

\footnotetext{
${ }^{18}$ Recall that $C_{a b c d}^{-}$is antiselfdual in both pairs of indices.
} 


\subsubsection{Special Geometry in Special Coordinates}

The next step is to eliminate the auxiliary fields by their equations of motion and to obtain an on-shell formulation of the theory in terms of unconstrained physical fields. This can be done in closed form for the minimal terms, i.e. as long as the chiral background $\widehat{\mathbf{A}}=\mathbf{W}^{2}$ is absent. Therefore we first restrict ourselves to a discussion of the minimal terms related to the prepotential $F\left(X^{I}, \widehat{A}=0\right)=F^{(0)}\left(X^{I}\right)$ and comment on the higher derivative terms later on. We will see that the target space of the scalar sigma model is restricted to be a special Kähler manifold, and that the structure of the whole theory is governed by special geometry 83, 17, 16.

The physical fields of the theory are the graviton $e_{\mu}{ }^{a}$, two gravitini $\psi_{\mu}^{i}, N_{V}+1$ gauge fields $W_{\mu}^{I}$, and $N_{V}$ gaugini-doublets and scalars described by $\Omega_{i}^{I}$ and $X^{I}$, subject to one complex constraint. Finding an on-shell formulation in terms of unconstrained fields includes the following: The auxiliary fields $T_{a b}^{i j}, \chi^{i}, D$ and $Y_{i j}^{I}$ have to be eliminated, as well as the gauge fields $b_{\mu}, A_{\mu}, \mathcal{V}_{\mu}{ }^{i}{ }_{j}$ of the local dilatational and $U(1) \otimes S U(2)$ gauge symmetry and the scalars and fermions have to be expressed in terms of unconstrained fields. We will only discuss the purely bosonic part of the Lagrangian. The fields $b_{\mu}, D$ have already been dealt with by imposing the $K$-gauge and by coupling to the non-linear multiplet, respectively. Moreover it turns out that $\mathcal{V}_{\mu}{ }^{i}{ }_{j}$ and $Y_{i j}^{I}$ are only relevant for fermionic terms.19 It remains to deal with the $U(1)$ connection $A_{\mu}$ and with the auxiliary field $T_{a b}^{i j}$.

Solving the equation of motion for the $U(1)$ gauge field gives 20

$$
A_{\mu}=\frac{1}{2} \frac{\bar{F}_{I} \stackrel{\leftrightarrow}{\partial}_{\mu} X^{I}-\bar{X}^{I} \stackrel{\leftrightarrow}{\partial}_{\mu} F_{I}}{-i\left(X^{I} \bar{F}_{I}-F_{I} \bar{X}^{I}\right)}
$$

In this formula neither the $D$ - nor the $A$-gauge has been imposed. Note that the field has Weyl and chiral weight 0 and therefore is gauge invariant. The above formula is used when working in the superconformal setup. A gauge fixed version is obtained by imposing the $D$-gauge, which sets the denominator to unity. In absence of a chiral background we can use the homogenity properties $F_{I}=F_{I J} X^{J}$ and $F_{I J K} X^{K}=0$ of the prepotential to rewrite the numerator and we get:

$$
A_{\mu}=\frac{i}{2} N_{I J}\left(X^{I} \partial_{\mu} \bar{X}^{J}-\bar{X}^{I} \partial_{\mu} X^{J}\right)
$$

As a result the scalar kinetic term

$$
8 \pi e^{-1} \mathcal{L}_{\text {scalar }}=i \mathcal{D}_{\mu} F_{I} \mathcal{D}^{\mu} \bar{X}^{I}+\text { h.c. }
$$

takes the form

$$
8 \pi e^{-1} \mathcal{L}_{\text {scalar }}=-N_{I J} \partial_{\mu} X^{I} \partial^{\mu} \bar{X}^{J}+\frac{1}{4}\left(N_{I J}\left(X^{I} \partial_{\mu} \bar{X}^{J}-\bar{X}^{I} \partial^{\mu} X^{J}\right)\right)^{2},
$$

where we again used homogenity properties (in particular that the $D$-gauge takes the form $\left.N_{I J} X^{I} \bar{X}^{J}=-1\right)$.

One could procede fixing the $U(1)$ gauge invariance by a suitable gauge condition and then solve the two real gauge conditions in terms of $N_{V}$ unconstrained scalars. In practice it is more convenient to leave the local $U(1)$ invariance intact. This is related to the fact that scalar fields in a locally supersymmetric theory are described by non-linear sigma models with a Kähler manifold as target space. ${ }^{27}$ Such models have an invariance under Kähler transformations, which are closely related to the local $U(1)$ transformations. Therefore it is natural to leave this symmetry intact. The corresponding gauge field is a composite field given by (3.124).

There is an elegant way of reformulating the theory in terms of $N_{V}$ unconstrained scalars (modulo local $U(1)$ transformations). It is based on the observation that the $D$-gauge condition has a geometrical interpretation, because it is a normalization condition for the scalars

\footnotetext{
${ }^{19}$ The equations of motion of $\mathcal{V}_{\mu}{ }^{i}{ }_{j}$ also involve hypermultiplet scalars, but we are concerned with the vector multiplets, only

${ }^{20}$ In chapter 2 the graviphoton was denoted $A_{\mu}$. In this chapter $A_{\mu}$ denotes the composite $U(1)$ connection, which is a completely different object. The graviphoton will only enter via its field strength in the following.

${ }^{21}$ In the case of abelian vector multiplets in $\mathcal{N}=2$ supergravity no scalar potential is possible.
} 
$X^{I}$. One interprets the $X^{I}$ as homogenous coordinates of the scalar manifold and introduces inhomogenous coordinates by

$$
Z^{0}=1=\frac{X^{0}}{X^{0}}, \quad Z^{A}=\frac{X^{A}}{X^{0}},
$$

where $A=1, \ldots, N_{V}$. Since these equations are $U(1)$ invariant one can use them to reconstruct the $X^{I}$ from a given set of inhomogenous coordinates $Z^{A}$ up to an irrelevant phase. The $Z^{A}$ are called special coordinates of the scalar manifold, because they provide a coordinatization that is distinguished by its relation to the $\mathcal{N}=2$ multiplet structure. General coordinatizations will be discussed in the next section.

We can now rewrite the scalar kinetic term using the unconstrained fields $Z^{A}$. First note that one can use the homogenity of $F\left(X^{I}\right)$ to rewrite it as a function of the $Z^{I}$ :

$$
F\left(X^{0}, \ldots, X^{N_{V}}\right)=\left(X^{0}\right)^{2} F\left(1, Z^{1}, \ldots, Z^{N_{V}}\right)=:\left(X^{0}\right)^{2} \widetilde{F}\left(Z^{1}, \ldots, Z^{N_{V}}\right) .
$$

For simplicity we will not introduce a new symbol for the prepotential as a function of $Z^{I}$ and simply write $F(Z)$ instead of $\widetilde{F}(Z)$. Since the $r$-th derivative is homogenous of degree $2-r$ we can likewise rewrite them:

$$
F(X)=\left(X^{0}\right)^{2} F(Z), \quad F_{I}(X)=X^{0} F_{I}(Z), \quad F_{I J}(X)=F_{I J}(Z),
$$

etc.

It is useful to introduce the matrix

$$
\mathcal{M}_{I \bar{J}}:=N_{I J}+N_{I K} \bar{X}^{K} N_{J L} X^{L}
$$

which has two null directions that are identified by the transversality equations

$$
X^{I} \mathcal{M}_{I \bar{J}}=0=\mathcal{M}_{I \bar{J}} \bar{X}^{J} .
$$

Using this matrix the scalar kinetic term can be rewritten

$$
8 \pi e^{-1} \mathcal{L}_{\text {scalar }}=-\mathcal{M}_{I \bar{J}} \partial_{\mu} X^{I} \partial^{\mu} \bar{X}^{J}
$$

as can be verified using homogenity and the $D$-gauge (note in particular $N_{I J} X^{I} \bar{X}^{J}=-1$ combined with $F_{I J K} X^{K}=0$ implies $\left.N_{I L} X^{L} \partial_{\mu} \bar{X}^{I}=-N_{I L} \bar{X}^{L} \partial_{\mu} X^{I}\right)$. Using the transversality equations this implies

$$
8 \pi e^{-1} \mathcal{L}_{\text {scalar }}=\left(Z^{K} N_{K L} \bar{Z}^{L}\right)^{-1} \mathcal{M}_{I \bar{J}} \partial_{\mu} Z^{I} \partial^{\mu} \bar{Z}^{J},
$$

where we used the $D$-gauge

$$
N_{I J} Z^{I} \bar{Z}^{J}=-\left|X^{0}\right|^{-2} .
$$

$\mathcal{M}_{I \bar{J}}$ can be expressed in terms of $Z^{I}$ as

$$
\mathcal{M}_{I \bar{J}}=N_{I J}-\frac{N_{I K} \bar{Z}^{K} N_{J L} Z^{L}}{Z^{M} N_{M N} \bar{Z}^{N}} .
$$

Then it is easy to show that

$$
K(Z, \bar{Z})=-\log \left(-N_{I J} Z^{I} \bar{Z}^{J}\right)=-\log \left(-i\left(Z^{I} \bar{F}_{I}-\bar{Z}^{I} F_{I}\right)\right)
$$

is a Kähler potential for the scalar metric, because

$$
\partial_{I} \partial_{\bar{J}} K=-\left(Z^{K} N_{K L} \bar{Z}^{L}\right)^{-1} \mathcal{M}_{I \bar{J}} .
$$


Therefore the scalar kinetic term is a Kähler sigma model:

$$
8 \pi e^{-1} \mathcal{L}_{\text {scalar }}=-g_{I} \partial_{\mu} Z^{I} \partial^{\mu} \bar{Z}^{J}, \quad g_{I \bar{J}}=\partial_{I} \partial_{\bar{J}} K .
$$

The target space of a scalar sigma model coupled to supergravity is not only restricted to be Kähler, but has to be Kähler-Hodge. This means that the Kähler metric comes from the fibre metric of a $U(1)$ bundle over the scalar manifold. The sigma model found above is of that type and the scalars $X^{I}$ are, speaking geometrically, sections of this $U(1)$ bundle. We will discuss this in the next section, when we investigate the intrinsic structure of the scalar manifold.

The Riemann curvature tensor of the scalar metric takes a remarkably simple form:

$$
R_{B C}^{A}=-2 \delta_{\left(B^{A} \delta^{D}\right)}^{D}-e^{2 K} Q_{B C E} \bar{Q}^{E A D},
$$

where

$$
Q_{A B C}:=i F_{I J K}(X(Z)) \frac{\partial X^{I}(Z)}{\partial Z^{A}} \frac{\partial X^{J}(Z)}{\partial Z^{B}} \frac{\partial X^{K}(Z)}{\partial Z^{C}} .
$$

A Kähler-Hodge manifold where the curvature tensor takes the special form (3.140) is called a special Kähler manifold. The above construction shows that in $\mathcal{N}=2$ Poincaré supergravity the geometry of the scalar manifold is even more restricted then in the case $\mathcal{N}=1$. This is so because vector multiplets contain both scalars and gauge fields. We will see in the next section how the additional geometric structure is related to symplectic invariance.

We now consider the vector kinetic term and the auxiliary $T$-field. The corresponding terms in the Lagrangian can be reorganized in the following way:

$$
8 \pi e^{-1} \mathcal{L}_{\mathrm{vect}}=\frac{i}{4} F_{I J} F_{a b}^{-I} F^{-J a b}+\frac{i}{8}\left(\bar{F}_{I}-F_{I J} \bar{X}^{J}\right) F_{a b}^{-I} T^{-a b}-\frac{1}{64} N_{I J} \bar{X}^{I} \bar{X}^{J} T_{a b}^{-} T^{-a b}+\text { h.c. } .
$$

We can now solve for the auxiliary $T$-field:

$$
T_{a b}^{-}=4\left(\bar{X}^{K} N_{K L} \bar{X}^{L}\right)^{-1} N_{I J} \bar{X}^{J} F_{a b}^{-I} .
$$

Plugging this back into the Lagrangian we get

$$
8 \pi e^{-1} \mathcal{L}_{\text {vect }}=\frac{i}{4} \overline{\mathcal{N}}_{I J} F_{a b}^{-I} F^{-J a b}-\frac{i}{4} \mathcal{N}_{I J} F_{a b}^{+I} F^{+J a b},
$$

where

$$
\mathcal{N}_{I J}=\bar{F}_{I J}+i \frac{N_{I K} Z^{K} N_{J L} Z^{L}}{Z^{M} N_{M N} Z^{N}} .
$$

After the elimination of the auxiliary $T$-field the dual gauge field takes the form

$$
G_{I a b}^{-}=\overline{\mathcal{N}}_{I J} F_{a b}^{-J} .
$$

Comparing to the bosonic off-shell Lagrangian we see that all terms linear in the field strengths become quadratic and the field dependent coupling and $\Theta$-angles take a more complicated form than in the off-shell formulation. But the theory still has symplectic reparametrization invariance. In particular the matrix $\mathcal{N}_{I J}$ transforms by projective transformations, so that formulae of section 3.4 carry over. Note that the matrix $\mathcal{N}_{I J}$ is invertible, because all the $N_{V}+1$ field strengths are independent.

The gauge field sector of the Poincaré theory is more complicated because one linear combination of the gauge fields belongs to the gravity multiplet. In the superconformal setup the $N_{V}+1$ field strengths together with their duals form a symplectic vector, whereas the Weyl multiplet is invariant. In the Poincaré theory the gravity supermultiplet has to be invariant and therefore the graviphoton is given by the symplectically invariant combination

$$
T_{a b}^{\mathrm{GP}-}=F_{I} F_{I a b}^{-}-X^{I} G_{I a b}^{-} .
$$

In the off-shell formulation the auxiliary field $T_{a b}^{-}$satisfies formally the same relation and is therefore often simply called the graviphoton. Note however that in the off-shell formalism the 
definition of $G_{I a b}$ depends itself on $T_{a b}^{-}$and therefore the relation is implicit. Moreover in the presence of $R^{2}$-terms the auxiliary $T$-field cannot be solved for explicitly but only iteratively. Therefore the relation between $T$-field and graviphoton is complicated.

The graviphoton field of $\mathcal{N}=2$ supergravity gauges the central charge transformations. The associated conserved charge

$$
Z=\frac{1}{4 \pi} \oint T^{-}=\lim _{r \rightarrow \infty} \frac{1}{4 \pi} \int_{0}^{2 \pi} \int_{0}^{\pi} T_{23}^{-} r^{2} \sin \theta d \theta d \phi
$$

is the central charge of the $\mathcal{N}=2$ supersymmetry algebra [78].

\subsubsection{Special Geometry in General Coordinates}

The special coordinates $Z^{A}$ are non-generic holomorphic coordinates, singled out by their relation to the $\mathcal{N}=2$ vector supermultiplets. In order to analyse intrinsic geometric properties of the scalar manifold we now introduce a set $z^{A}$ of generic local holomorphic coordinates. The special coordinates, being a specific system of holomorphic coordinates, are holomorphically related to them, $Z^{I}=Z^{I}(z)$.

Let us first clarify the relation between local $U(1)$ transformations and Kähler transformations, following [117]. The scalars $X^{I}$ transform as follows under local scale $(D)$ and $U(1)(A)$ transformations:

$$
X^{I} \rightarrow e^{\Lambda_{D}(z)-i \Lambda_{A}(z)} X^{I}
$$

One can split the $X^{I}$ as

$$
X^{I}=a X^{I}(z),
$$

which introduces a holomorphic ambiguity

$$
X^{I}(z) \rightarrow e^{\Lambda(z)} X^{I}(z), \quad a \rightarrow e^{-\Lambda(z)} a .
$$

The motivation behind the notation $X^{I}(z)$ will become obvious later. The extra symmetry (3.151) can be used to take $X^{I}(z)$ to be invariant under scale and $U(1)$ transformations. The $D$-gauge implies $|a|^{2}=e^{K}$, where

$$
K(z, \bar{z})=-\log \left(-i\left[X^{I}(z) \bar{F}_{I}(\bar{X}(\bar{z}))-F_{I}(X(z)) \bar{X}^{I}(\bar{z})\right]\right) .
$$

The $U(1)$ invariance can be fixed by choosing $a$ to be real and positive, $a=e^{K / 2}$ :

$$
X^{I}=e^{\frac{1}{2} K(z, \bar{z})} X^{I}(z) .
$$

After that, there is still a residual invariance under combined $A$ and holomorphic transformations

$$
-i \Lambda_{A}(z)=\frac{1}{2}(f(z)-\bar{f}(\bar{z})), \quad \Lambda(z)=f(z),
$$

which act by

$$
X^{I} \rightarrow e^{i \operatorname{Im} f(z)} X^{I}, \quad a \rightarrow e^{-\operatorname{Re} f(z)} a, \quad X^{I}(z) \rightarrow e^{f(z)} X^{I}(z) .
$$

This invariance can be fixed by imposing a constraint on the $X^{I}(z)$. One possible choice is to take them to be special coordinates,

$$
X^{0}(z)=Z^{0}=1 \text { and } X^{A}(z)=Z^{A} .
$$

Comparing to (3.137) we see that the function $(3.152)$ is the Kähler potential of the scalar sigma model.

We could instead impose another condition, but since we know that the scalar manifold is a holomorphic hypersurface the constraint must take the form $g\left(X^{I}(z)\right)=$ const, with holomorphic $g$. Changing the condition amounts to a holomorphic reparametrization of the 
hypersurface, $X^{I}(z) \rightarrow e^{f(z)} X^{I}(z)$, which acts on the $X^{I}$ as a local $U(1)$ transformation, according to 3.155$)$. Note that the $X^{I}(z)$ are holomorphically related to the generic holomorphic coordinates $z^{A}$. This motivates the notation we have chosen.

The transformation (3.155) acts on the Kähler potential as a Kähler transformation

$$
K(z, \bar{z}) \rightarrow K-f-\bar{f},
$$

which leaves the metric of the scalar sigma model invariant. This is the link between local $U(1)$ transformations and Kähler transformations that we promised to explain.

The next step is to find out how the theory behaves under a general holomorphic reparametrization $z \rightarrow z^{\prime}(z)$. This way one can arrive at an intrinsic characterization of special Kähler geometry and see that the fields $X^{I}$ and $X^{I}(z)$ can be geometrically characterized as sections of certain bundles over the scalar manifold.

Note that in the minimal coupling case the knowledge of the symplectic vector $\left(X^{I}, F_{J}\right)$ is sufficient to write down the on-shell action. In the absence of the chiral background $2 F=$ $F_{I} X^{I}$ and therefore we can take $\left(X^{I}, F_{J}\right)$ as the defining data.2 Using the newly defined $X^{I}(z)$ and the homogenity of the prepotential we can likewise describe it by $\left(X^{I}(z), F_{J}(X(z))\right)$. Since the form of the action cannot depend on how we parametrize the scalar manifold, a reparametrization $z \rightarrow z^{\prime}$ must yield a new vector $\left(X^{I}\left(z^{\prime}\right), F_{J}\left(X\left(z^{\prime}\right)\right)\right)$. The most general way in which the two vectors can be related is a combination of a Kähler transformation with a symplectic transformation:

$$
\left(\begin{array}{c}
X^{I}\left(z^{\prime}\right) \\
F_{J}\left(X\left(z^{\prime}\right)\right)
\end{array}\right)=e^{f(z)} \mathcal{O}_{J K}^{I L}\left(\begin{array}{c}
X^{K}(z) \\
F_{L}(X(z))
\end{array}\right) .
$$

For $\left(X^{I}, F_{J}\right)$ the Kähler transformation acts by the induced $U(1)$ transformation:

$$
\left(\begin{array}{c}
\left(X^{I}\right)^{\prime} \\
\left(F_{J}\right)^{\prime}
\end{array}\right)=e^{i \operatorname{Im} f(z)} \mathcal{O}_{J K}^{I L}\left(\begin{array}{c}
X^{K} \\
F_{L}
\end{array}\right) .
$$

This provides a geometric characterization of the symplectic vectors: $\left(X^{I}(z), F_{J}(X(z))\right)$ is a section of a bundle $\mathcal{L} \otimes \mathcal{H}$ over the scalar manifold, where $\mathcal{L}$ is a holomorphic line bundle and $\mathcal{H}$ is a flat symplectic vector bundle. $\left(X^{I}, F_{J}\right)$ is a section of a related bundle $\mathcal{P} \otimes \mathcal{H}$, where $\mathcal{P}$ is the principal $U(1)$ bundle associated to $\mathcal{L}$.

We now collect how various terms in the Lagrangian look, when expressed in terms of general coordinates. The scalar kinetic term is a Kähler sigma-model,

$$
e^{-1} \mathcal{L}_{\text {scalar }} \sim-g_{A} \bar{B}_{\mu} z^{A} \partial^{\mu} \bar{z}^{\bar{B}}, \quad g_{A \bar{B}}=\partial_{A} \partial_{\bar{B}} K(z, \bar{z}),
$$

with Kähler potential

$$
K=-\log \left(-i\left[X^{I}(z) \bar{F}_{I}(\bar{X}(\bar{z}))-F_{I}(X(z)) \bar{X}^{I}(\bar{z})\right]\right)
$$

and the vector kinetic term is

$$
e^{-1} \mathcal{L}_{\text {vect }} \sim \frac{i}{4} \overline{\mathcal{N}}_{I J} F_{a b}^{-I} F^{-I a b}-\frac{i}{4} \mathcal{N}_{I J} F_{a b}^{I+} F^{I+a b},
$$

with

$$
\mathcal{N}_{I J}=\bar{F}_{I J}+i \frac{N_{I K} X^{K}(z) N_{J L} X^{L}(z)}{X^{M}(z) N_{M N} X^{N}(z)} .
$$

\footnotetext{
${ }^{22}$ As we will see later, there exist vectors of the form $\left(X^{I}, F_{J}\right)$, which are not related to a prepotential $F(X)$. But it turns out that such choices are related by symplectic transformations to symplectic vectors which come from a prepotential. Also note that the existence of a prepotential is indispensable for the off-shell formulation. Only after elimination of the auxiliary fields the theory can be formulated purely in terms of $\left(X^{I}, F_{J}\right)$.
} 
In order to define a physical model the section $\left(X^{I}(z), F_{J}(z)\right)$ has to be chosen such that the various kinetic terms are positive definite. From the spin-two, spin-one and spin-zero kinetic terms one gets three conditions:

$$
-i\left(X^{I}(z) \bar{F}_{I}\left(\bar{X}(\bar{z})-F_{I}(X(z)) \bar{X}^{I}(\bar{z})\right)>0,\right.
$$

and the matrices $\operatorname{Im} \mathcal{N}_{I J}$ and $g_{A \bar{B}}(z, \bar{z})$ must be negatvie definite and positive definite, respectively. It can be shown that the second condition is implied by the first and third [17].

Let us investigate the bundles $\mathcal{L}$ and $\mathcal{P}$ a little closer [97]. A section $\phi(z, \bar{z})$ of $\mathcal{L}^{p}$, the $p$-th power of $\mathcal{L}$ is an object that transforms according to

$$
\phi(z, \bar{z}) \rightarrow e^{p f(z)} \phi(z, \bar{z}) .
$$

A natural connection on $\mathcal{L}^{p}$ is found by covariantizing the complex partial derivatives with respect to the Kähler transformations:

$$
D_{A} \phi=\left(\partial_{A}+p\left(\partial_{A} K\right)\right) \phi, \quad D_{\bar{A}} \phi=\partial_{\bar{A}} \phi .
$$

Note that for $\mathcal{L}^{p}$ covariantly holomorphic is the same as holomorphic, $D_{\bar{A}} \phi=\partial_{\bar{A}} \phi=0$. The sections $X^{I}(z)$ of $\mathcal{L}$ are holomorphic, $\partial_{\bar{A}} X^{I}(z)=0$.

Sections $\varphi(z, \bar{z})$ of $\mathcal{P}^{p}$, the $p$-th power of the associated principal $U(1)$ bundle transform as

$$
\varphi(z, \bar{z}) \rightarrow e^{i p \operatorname{Im} f(z)} \varphi(z, \bar{z}) .
$$

Such sections can be obtained from sections of $\mathcal{L}^{p}$ by $\varphi=e^{p / 2 K} \phi$. The $U(1)$ covariant derivatives are

$$
D_{A}^{\prime} \varphi=\left(\partial_{A}+\frac{p}{2}\left(\partial_{A} K\right)\right) \varphi, \quad D_{\bar{A}}^{\prime} \varphi=\left(\partial_{\bar{A}}-\frac{p}{2}\left(\partial_{\bar{A}} K\right)\right) \varphi .
$$

A section of $\mathcal{P}^{p}$ is covariantly holomorphic, $D_{\bar{A}}^{\prime} \varphi=0$ if and only if the corresponding section of $\mathcal{L}^{p}$ is holomorphic. In particular the sections $X^{I}$ of $\mathcal{P}$ are covariantly holomorphic.

The bundles $\mathcal{L}$ and $\mathcal{P}$ are nontrivial and the connections introduced above can be used to compute their first Chern classes, or, in physical terms, the integrated field strength associated with the Kähler gauge field.

The connection one-form of the holomorphic connection on $\mathcal{L}$ is $\Theta=\partial K=\partial_{A} K d z^{A}$, and the associated connection one-form $Q$ on $\mathcal{P}$ is the imaginary part $Q=\operatorname{Im} \Theta$. By definition the first Chern classes are the $H^{2}(M, \mathbf{R})_{\text {de Rham }}$ cohomology classes of the appropriately normalized curvatures ( $M$ denotes the scalar manifold):

$$
c_{1}(\mathcal{L})=2 \pi i[\bar{\partial} \Theta] .
$$

The Kähler two-form $\mathbf{K}$ of the scalar manifold $M$ is

$$
\mathbf{K}=\frac{i}{2 \pi} g_{A \bar{B}} d z^{A} \wedge d \bar{z}^{\bar{B}}=\frac{i}{2 \pi} \partial \bar{\partial} K .
$$

The curvature of $\mathcal{L}$ and the Kähler two-form are related by

$$
2 \pi i \bar{\partial} \Theta=\mathbf{K}
$$

and therefore the Kähler class is integral:

$$
[\mathbf{K}]=c_{1}(\mathcal{L}) .
$$

Kähler manifolds with this property are called Kähler-Hodge manifolds in the mathematical literature. They have the particular property that the Kähler metric comes from the fibre metric of a holomorphic line bundle $\mathcal{L}$ over the manifold. The fibre metric on $\mathcal{L}_{z}$ is just the exponential $e^{K}$ of the Kähler potential at fixed $z$. Alternatively one can express this in terms of the associated $U(1)$ principal bundle which has the curvature

$$
d Q=2 \pi \mathbf{K} .
$$


In the physics literature a manifold is called Kähler-Hodge if it is an admissible scalar manifold in $\mathcal{N}=1$ supergravity. ${ }^{23}$ This is more restrictive than the mathematical definition, because of the presence of fermions which are sections of $\mathcal{L}^{1 / 2}$. Kähler invariance in the presence of fermions requires a compensating chiral rotation, which is only well defined if the curvature on $\mathcal{L}^{1 / 2}$ is normalized as $\int F=2 \pi i n$, with $n \in \mathbf{Z}$. (In mathematical terms this means that one can take the square root of $\mathcal{L}$ such that $\mathcal{L}^{1 / 2}$ is a well defined holomorphic line bundle.) As a consequence the Kähler form must have even integer cohomology and not just integer cohomology, because $[\mathbf{K}]=2 c_{1}\left(\mathcal{L}^{1 / 2}\right)$ [117.

We finally note that the Kähler connection one-form $Q$ is related to the $U(1)$ connection constructed earlier. In (3.124) the $U(1)$ connection was expressed in terms of $\left(X^{I}, F_{J}\right)$ by its equation of motion. Rewriting this in terms of $\left(X^{I}(z), F_{J}(X(z))\right)$ we find

$$
A_{\mu}=\frac{1}{2} e^{K}\left(\bar{F}_{I}(\bar{X}(\bar{z})) \overleftrightarrow{\partial}_{\mu} X^{I}(z)-\bar{X}^{I}(\bar{z}) \overleftrightarrow{\partial}_{\mu} F^{I}(X(z))\right)
$$

The connection one-form $Q$ is related to a composite space-time gauge field by

$$
Q_{\mu}=-\frac{i}{2}\left(\partial_{A} K \partial_{\mu} z^{A}-\partial_{\bar{A}} K \partial_{\mu} \bar{z}^{\bar{A}}\right)
$$

Using

$$
\partial_{A} K \partial_{\mu} z^{A}=i e^{K}\left(\bar{F}_{I}(\bar{X}(\bar{z})) \partial_{\mu} X^{I}-\bar{X}^{I} \partial_{\mu} F_{I}(X(z))\right)
$$

we find $A_{\mu}=Q_{\mu}$.

So far we have focussed on the bundles $\mathcal{L}, \mathcal{P}$ related to the Kähler-Hodge structure required by a consistent coupling to $\mathcal{N}=1$ supergravity. We next turn to the symplectic bundle $\mathcal{H}$ which is the additional structure required by coupling vector multiplets to $\mathcal{N}=2$ supergravity. Here scalars and vectors sit in the same multiplet and the consistent action of symplectic reparametrizations puts additional restrictions on the scalar manifold. The admissible manifolds of vector multiplet scalars in $\mathcal{N}=2$ supergravity are called special Kähler manifolds. We already gave a definition in terms of the Riemann curvature tensor in special coordinates. The first intrinsic definition was given by Strominger [119]: A Kähler-Hodge manifold is special Kähler if it allows a flat symplectic vector bundle $\mathcal{H}$ with a holomorphic section $v, \bar{\partial} v=0$, such that the Kähler form $\mathbf{K}$ can be expressed in terms of the section as

$$
\mathbf{K}=-i \partial \bar{\partial} \log (-i\langle\bar{v}, v\rangle)
$$

where $\langle u, w\rangle=u^{T} \Omega w$ is the symplectic bilinear form. As pointed out in [117 one has to include the additional condition

$$
\left\langle v, \partial_{A} v\right\rangle=0
$$

in order to guarantee that the matrix $\mathcal{N}_{I J}$ is symmetric: for models constructed using KaluzaKlein compactification on Calabi-Yau-threefolds this holds automatically, but it is not guaranteed in general.

Various other definitions can be given. For example one can work with the principal bundle $\mathcal{P}$ instead of the line bundle $\mathcal{L}$. Then holomorphicity conditions are replaced by covariant holomorphicity properties. Other definitions use the characterization through the curvature tensor or the existence of special coordinates. We will not enter the details here but refer to 117, 97. More about the geometric formulation of $\mathcal{N}=2$ supergravity can be found in 95, 96, 98.

Finally we have to discuss one further point. The intrinsic definition reviewed above does not directly refer to a prepotential, but only required the existence of a secion $v$ of $\mathcal{L} \otimes \mathcal{H}$ with certain properties. It is not true that all such sections take the form $\left(X^{I}(z), F_{J}(X(z))\right)$, where $X^{I}(z)$ are coordinates and $F_{J}(X(z))$ is the gradient of a prepotential [120]. For instance, if one starts with a section with prepotential and performs a symplectic transformation where the new $\breve{X}^{I}(z)$ are not invertibly related with the old $X^{I}(z)$,

$$
\operatorname{det} \frac{\partial \breve{X}^{I}}{\partial X^{J}}=0
$$

\footnotetext{
${ }^{23}$ We refer to [118] for a review of $\mathcal{N}=1$ supergravity, its matter couplings and its geometric structure.
} 
then one finds that the new would-be prepotential vanishes identically, $\breve{F}=0$. Note that in the superconformal case the $X^{I}$ were treated as $N_{V}+1$ independent scalars, so that it was natural to require that symplectic transformations are related to invertible transformations of the scalars. In the super Poincaré situation, where only $N_{V}$ scalars are independent, there is no reason for imposing this condition. As a consequence models exist which cannot be described by a prepotential. Nevertheless it can be shown that every such model can be reparametrized by a symplectic transformation such that in the new basis a prepotential exists. In fact, the existence of a description through special coordinates and a prepotential provides one of the possible definitions of special Kähler geometry [117]. We will see later that for some models obtained from string theory the description in a basis without prepotential is the natural one. Finally we would like to point out that the equivalence of models without prepotential to models with prepotential requires the possibility of symplectic reparametrizations. If these are no longer possible, for example when considering gauged supergravity, then the equivalence cannot be expected to hold.

\subsubsection{Consequences of the Presence of Higher Derivative Terms}

In the last two sections we discussed the minimal terms of the action, which are controlled by the prepotential $F^{(0)}$. Non-minimal higher derivative terms are encoded in the higher terms $F^{(g>0)}(X)$ of the function $F\left(X, \widehat{A}=W^{2}\right)$. Once these terms are taken into account several things change. The first thing is that the Lagrangian becomes much more complicated. In particular one cannot eliminate the auxiliary fields in closed form. A closer look shows that the Lagrangian now contains derivatives of the auxiliary fields, which asks for an interpretation. Auxiliary fields are expected to have algebraic equations of motion, so that they do not introduce new degrees of freedom into the theory. We already discussed in chaper 2 that the $R^{2}$-terms themselves also introduce new degrees of freedom which are in conflict with perturbative unitarity. The resolution of the problem is the same in both cases: The Lagrangian is not to be interpreted as a fundamental Lagrangian but as an effective Lagrangian of the underlying fundamental theory that we believe to be string theory. The expansion in terms of derivatives is a low energy expansion, because every derivative is suppressed by a factor of $m_{\text {Planck }}$. The effective action is used to compute corrections iteratively, oder by order in $m_{\text {Planck }}$. The fundamental theory has to provide the explicit form of the functions $F^{(g)}(X)$. As we will see later these quantities can be computed in string perturbation theory.

Since the auxiliary fields can only be solved for iteratively we have to insist on the existence of a prepotential. As we explained in the last section, this is no loss in generality at least as long as we consider ungauged supergravity. In view of the complicated dependence of the Lagrangian on the auxiliary fields, we will try to avoid using its explicit form as much as we can. In particular we will not eliminate the auxiliary fields, and we will work in the superconformal setup where symmetries are realized in a more simple way. The computation of black hole entropy will show that this is an effective way for solving problems explicitly. 


\section{Chapter 4}

\section{Four-Dimensional $\mathcal{N}=2$ Black Holes}

In this chapter we discuss extremal black holes in $\mathcal{N}=2$ supergravity with $N_{V}+1$ vector multiplets. The discussion is model-independent, because we work with a general function $F(X, \widehat{A})$. We derive one of our main results, the model-independent entropy formula (4.84). This result and its derivation were briefly described in the letter [91. Here we will give a detailed presentation. The main line of thought is the following: As we discussed in chapter 2 the extremal Reissner-Nordstrøm black hole is a BPS-soliton which interpolates between two $\mathcal{N}=2$ supersymmetric vacua, flat space at infinity and $A d S^{2} \times S^{2}$ at the horizon [10]. The same is true when considering $\mathcal{N}=2$ supergravity coupled to vector multiplets with a general prepotential $F^{(0)}(X)$ : The event horizon of a static and spherically symmetric BPS black hole is fully $\mathcal{N}=2$ supersymmetric and the solution therefore interpolates between two $\mathcal{N}=2$ vacua 121, 122, 123, 124]. Naturally one expects that this property will persist when $R^{2}$-terms are switched on. One can give a general argument for this: In the sections 4.2.2 and 4.3 we will review how the asymptotic behaviour of BPS black holes is determined by the so-called stabilization equations. Symplectic covariance requires that these equations have to be modified in presence of $R^{2}$-terms [125, 91]. The modified stabilization equations then imply universal behaviour and full $\mathcal{N}=2$ supersymmetry on the horizon.

In absence of $R^{2}$-terms one can derive the stabilization equations from the BPS condition. Moreover one can find an expression for the full interpolating black hole solution, which is determined by the generalized stabilization equations [126, 127, 128]. The same is expected when $R^{2}$-terms are present and we will argue in section 4.3 that the result is predicted by symplectic invariance. A derivation of the stabilization equations and of the full black hole solution in presence of $R^{2}$-terms is currently under investigation and the result will be the subject of a future publication [19]. Here we will determine the near horizon geometry by imposing full $\mathcal{N}=2$ supersymmetry on a static and spherically symmetric field configuration. This is already a complicated problem and we will devote section 4.1 to a detailed discussion. The result is that the unique static and spherically symmetric $\mathcal{N}=2$ vacuum is the BertottiRobinson geometry $A d S^{2} \times S^{2}$. The geometry and the corresponding gauge and scalar fields can be expressed in terms of a single field $Z$, which is related to the central charge.

In section 4.2.1 we use Wald's entropy formular to compute the black hole entropy corresponding to our near horizon solution. We call the resulting formula model-independent, because it is valid for arbitrary $F\left(X^{I}, \widehat{A}\right)$. The formula is manifestly covariant under symplectic reparametrizations and the entropy is uniquely determined by the value of the field $Z$ at the horizon. This field is a function of the electric and magnetic charges and of the scalar fields.

In section 4.2 .2 we review the supersymmetric attractor mechanism and how it leads to the stabilization equations which determine the near horizon behaviour of the scalar fields in terms of the charges 121, 122, 123, 124. Based on symplectic invariance we propose a generalization of the stabilization equations to the case with $R^{2}$-terms 125,91 . Combining 
the stabilization equations with our model-independent entropy formula we see that the black hole entropy is determined by the charges. In order to arrive at explicit expressions one has to specify concrete models by an explicit choice of the function $F(X, \widehat{A})$ and then to solve the stabilization equations. This will be done in chapter 6 .

In section 4.3 we review for completeness the structure of the full explicit black hole solutions in $\mathcal{N}=2$ supergravity without $R^{2}$-terms. We explain how the the solution is expressed in terms of harmonic functions using the so-called generalized stabilization equations [126, 127, 128. As already mentioned the generalization of these results to the case with $R^{2}$-terms is currently under investigation [19]. Here we will restrict ourselves to a few remarks which are based on symplectic invariance.

\subsection{The Near Horizon Geometry}

We now turn to the classification of all fully supersymmetric, static and spherically symmetric field configurations of $\mathcal{N}=2$ supergravity with $N_{V}+1$ vector multiplets, based on a general function $F(X, \widehat{A})$, where $\widehat{A}$ will eventually be identified with lowest component of the Weyl multiplet $\mathbf{W}^{2}$.

By reparametrizations a static and spherically symmetric metric can be brought to the form

$$
d s^{2}=-e^{2 g(r)} d t^{2}+e^{2 f(r)}\left(d r^{2}+r^{2}\left(\sin ^{2} \theta d \phi^{2}+d \theta^{2}\right)\right)
$$

In absence of higher curvature terms it was shown by Tod [129] that for a superymmetric static metric the two functions $f$ and $g$ are not independent, but related through $f=-g$. We will not make the assumption that this generalizes to our case but rather prove it. Therefore in our ansatz (4.1) $f$ and $g$ are independent functions.

Since we are looking for bosonic field configurations which are invariant under all $\mathcal{N}=2$ supertransformations, nontrivial constraints arise from the condition that variations of fermions with arbitrary transformation parameter $\epsilon^{i}$ must vanish when evaluated in the background. The bosonic fields themselves vary into fermions, which by assumption vanish in the background. The vanishing of the fermionic variations imposes conditions on the bosonic background and we have to find the most general static and spherically symmetric bosonic background which satisfies them. Terms which are at least quadratic in the fermions are irrelevant for our problem because they vanish and transform under supersymmetry into objects which vanish in a bosonic background. Therefore we will save work in this chapter by systematically ignoring all higher order fermionic terms. This will be applied from now on without further notice.

We will work in the superconformal off-shell formulation and in a general chiral background field. This way we can avoid to deal with the complications of the action and the equations of motion that we described earlier. We require that the variations of all fermionic quantities vanish in the bosonic background for arbitrary choice of the supersymmetry parameter. As we will see the vanishing of the variation of a fermion does not necessarily imply that the variation of the covariant derivative of the fermion vanishes. For example the vanishing of the variation of the covariant derivatives of the gaugini gives new conditions on the background, which are equivalent to the field equations and Bianchi identities of the gauge fields. Therefore we have to continue analysing fermionic variations until no new conditions can arise, because the background is completely determined. Since we are imposing all conditions on the bosonic background which are compatible with $\mathcal{N}=2$ supersymmetry, we know that the background must satisfy the equations of motion. Thus by imposing full supersymmetry we do not need to solve the equations of motion directly. This is similar to the analysis of fully supersymmetric compactifications of eleven-dimensional supergravity in [130, where unbroken supersymmetry requires that the variation of the supercovariant gravitino field strength vanishes. In that case it follows from this single condition that all equations of motion are satisfied.

Since we keep superconformal invariance intact while solving the Killing spinor equations we profit from the simpler structure of the supersymmetry variations and from the fact that the superconformal off-shell multiplets are smaller then their Poincaré counterparts. In order 
to apply our results to black holes in $\mathcal{N}=2$ Poincare supergravity we finally have to fix the extra conformal symmetries or to consider suitable gauge invariant quantities. As we saw earlier dilation invariance is fixed by setting

$$
e^{-\mathcal{K}}:=i\left(F_{I}(X, \widehat{A}) \bar{X}^{I}-X^{I} \bar{F}_{I}(\bar{X}, \widehat{\widehat{A}})\right)
$$

to a constant. In the superconformal situation $e^{-\mathcal{K}}$ is a symplectic function of Weyl weight $w=2$ and chiral weight $c=0$. It appears in various places, for example in the Einstein-Hilbert term, in order to preserve dilatational invariance. We will use it to construct dilatation-invariant quantities. The notation $\mathcal{K}$ is chosen because this quantity resembles the Kähler potential. Note however that it does not reduce to the Kähler potential when setting $\widehat{A}=0$ because it involves the covariantly holomorphic section $\left(X^{I}, F_{J}\right)$ and not the holomorphic one, $\left(X^{I}(z), F_{J}(X(z))\right.$.

In the superconformal setup the special $S$-supertransformations are still present. Therefore we cannot require strict invariance under $Q$-supertransformations, but only $Q$-invariance up to an uniform $S$-supertransformation. An elegant way to deal with this is to find $S$-invariant spinors. This can be done using a spinor $\zeta_{i}$ which under $S$-supersymmetry transforms into the $S$-variation parameter $\eta_{i}$. Given any spinor that transforms under $S$, one can form a suitable combination with $\zeta_{i}$ such that the $S$-variations mutually cancel. A spinor with the required inhomogenous transformation under $S$ can be found and is related by supersymmetry to the function $\mathcal{K}$ :

$$
\zeta_{i}=-\left(\Omega_{i}^{I} \frac{\partial}{\partial X^{T}}+\widehat{\psi}_{i} \frac{\partial}{\partial \widehat{A}}\right) \mathcal{K}=-i e^{\mathcal{K}}\left(\left(\bar{F}_{I}-\bar{X}^{J} F_{I J}\right) \Omega_{i}^{I}-\bar{X}^{I} F_{I \widehat{A}} \widehat{\psi}_{i}\right) .
$$

The derivative operator acts on the symplectically invariant $\mathcal{K}$ in such a way that $\zeta_{i}$ is symplectically invariant. The behaviour of $\zeta_{i}$ under $Q$ - and $S$-transformations is

$$
\begin{aligned}
\delta \zeta_{i}= & -2 i e^{\mathcal{K}}\left(\bar{F}_{I} \gamma^{a} D_{a} X^{I}-\bar{X}^{I} \gamma^{a} D_{a} F_{I}\right) \epsilon_{i}-i e^{\mathcal{K}}\left(\left(\bar{F}_{I}-\bar{X}^{J} F_{J I}\right) Y_{i j}^{I}-\bar{X}^{I} F_{I \widehat{A}} \widehat{B}_{i j}\right) \epsilon^{j} \\
& -\frac{1}{2} i \varepsilon_{i j} \mathcal{F}_{a b}^{-} \gamma^{a b} \epsilon^{j}+2 \eta_{i},
\end{aligned}
$$

where we defined

$$
\mathcal{F}_{a b}^{-}:=e^{\mathcal{K}}\left(\bar{F}_{I} F_{a b}^{-I}-\bar{X}^{I} G_{a b I}^{-}\right) .
$$

Note that $\zeta_{i}$ has the required behaviour under $S$-transformations. Now we can form $S$-invariant spinors and require that their $Q$-variations vanish exactly.

\subsubsection{The Gaugino Variations}

We start our analysis with the gaugini. The $S$-invariant combination is given by $\Omega_{i}^{I}-X^{I} \zeta_{i}$. The $Q$ variation has to vanish:

$$
\begin{aligned}
\delta\left(\Omega_{i}^{I}-X^{I} \zeta_{i}\right)= & 2 D^{a} X^{I} \gamma_{a} \epsilon_{i}+\frac{1}{2} \varepsilon_{i j} \mathcal{F}^{-I a b} \gamma_{a b} \epsilon^{j}+Y_{i j}^{I} \epsilon^{j} \\
& +i e^{\mathcal{K}} X^{I}\left[2\left(\bar{F}_{J} D^{a} X^{J}-\bar{X}^{J} D^{a} F_{J}\right) \gamma_{a} \epsilon_{i}+\frac{1}{2}\left(\bar{F}_{J} F_{a b}^{-J}-\bar{X}^{J} G_{J a b}^{-}\right) \gamma^{a b} \varepsilon_{i j} \epsilon^{j}\right] \\
& +X^{I}\left(\left(\bar{F}_{J}-\bar{X}^{K} F_{K J}\right) Y_{i j}^{J}-\bar{X}^{J} F_{J \widehat{A}} \widehat{B}_{i j}\right) \epsilon^{j} \\
\stackrel{!}{=} & 0 .
\end{aligned}
$$

Since this must hold for all choices of $\epsilon^{i}$ the coefficients of terms with a different structure concerning spinor indices (which we did not write out explicitly) and $S U(2)$ indices $(i, j)$ have

\footnotetext{
${ }^{1}$ This corresponds to the fact that Poincaré $Q$-supertransformations are combinations of $Q$ - and $S$ supertransformations and $K$-transformations. For the problem studied in this chapter the $K$-transformations are not relevant, since all the quantities whose supersymmetry variation we require to vanish are $K$-invariant.
} 
to vanish separately. There are three independent types of terms: those proportional to $\gamma^{a} \epsilon_{i}$, to $\epsilon^{j}$ and to $\gamma_{a b} \varepsilon_{i j} \epsilon^{j}$. Thus we get three equations:

$$
\begin{aligned}
& D_{a} X^{I}+i e^{\mathcal{K}} X^{I}\left(\bar{F}_{J} D_{a} X^{J}-\bar{X}^{J} D_{a} F_{J}\right)=0, \\
& \mathcal{F}_{a b}^{I-}+i e^{\mathcal{K}} X^{I}\left(\bar{F}_{J} F_{a b}^{-J}-\bar{X}^{I} G_{I a b}^{-}\right)=0, \\
& Y_{i j}^{I}+i e^{\mathcal{K}} X^{I}\left(\left(\bar{F}_{J}-\bar{X}^{J} F_{J K}\right) Y_{i j}^{K}-\bar{X}^{J} F_{J \widehat{A}} \widehat{B}_{i j}\right)=0 .
\end{aligned}
$$

The first equation can be brought to a simpler form by writing out the covariant derivatives and reorganizing terms using the composite connection

$$
\mathcal{A}_{a}=\frac{1}{2} e^{\mathcal{K}}\left(\bar{X}^{J} \stackrel{\leftrightarrow}{\partial}_{a} F_{J}-\bar{F}_{J} \stackrel{\leftrightarrow}{\partial}_{a} X^{J}\right)
$$

Using this equation (4.7) takes the form

$$
\left(\partial_{a}-i \mathcal{A}_{a}\right)\left(e^{\mathcal{K} / 2} X^{I}\right)=0 .
$$

Note that this equation is Weyl and $U(1)$ invariant. Moreover it is obvious that there is an integrability condition

$$
\partial_{[a} \mathcal{A}_{b]}=0,
$$

which tells us that the connection $\mathcal{A}_{a}$ is flat. Using the quantity $\mathcal{F}_{a b}^{-}$introduced in (4.5) we can also write equation (4.8) in more suggestive form:

$$
\mathcal{F}_{a b}^{I-}=-i X^{I} \mathcal{F}_{a b}^{-} .
$$

\subsubsection{The Background Spinor Variation}

Next we look at the variation of the background spinor $\widehat{\psi}_{i}$. The structure is the same as for the gaugino variation. In a completely analogous way we get three equations. The first can be brought to the form

$$
\left(\partial_{a}-w i \mathcal{A}_{\mu}\right)\left(e^{w \mathcal{K} / 2} \widehat{A}\right)=0 .
$$

Combining this with (4.11) and using homogenity of $F(X, \widehat{A})$ we find

$$
\left(\partial_{a}-i \mathcal{A}_{a}\right)\left(e^{\mathcal{K} / 2} F_{I}\right)=0 .
$$

The second equation can be brought to the form

$$
\widehat{F}_{a b}^{-}=-i w \widehat{A} \mathcal{F}_{a b}^{-} \text {. }
$$

The third equation can be combined with (4.13) resulting in

$$
\widehat{B}_{i j} X^{I}=w \widehat{A} Y_{i j}^{I}
$$

\subsubsection{The Gravitini Variations}

We now turn to the variation of the gravitini $\psi_{a}^{i}$. Since the gravitini are gauge fields it is to restrictive to set them to zero, instead of requiring that they are pure gauge. Therefore we will only require that the corresponding gauge-invariant quantity, the gravitino field strength $R_{a b}^{i}(Q)$ has a vanishing $Q$-variation modulo an $S$-variation. As before we first have to find the appropriate $S$-invariant object, which is $R_{a b}^{i}(Q)-\frac{1}{16} T^{c d i j} \gamma_{c d} \gamma_{a b} \zeta_{j}$. The resulting expression is somewhat more complicated than the ones we encountered before. One can use the selfduality and chirality properties of the various quantities and the $\gamma$-matrix identities listed in appendix 
A to simplify it. After collecting terms with the same spinor and $S U(2)$ index structure the result takes the form

$$
\begin{aligned}
& \delta R_{a b}^{i}(Q)-\frac{1}{16} T^{c d i j} \gamma_{c d} \gamma_{a b} \delta \zeta_{j} \\
= & A_{a b}^{e} \gamma_{e} \varepsilon^{i j} \epsilon_{j}+B_{a b}^{c d} \sigma_{c d} \epsilon^{i}+\varepsilon^{i j} C_{a b j k} \epsilon^{k}+D_{a b j}^{c d i} \sigma_{c d} \epsilon^{j}+E_{a b} \epsilon^{i} \\
\stackrel{!}{=} & 0 .
\end{aligned}
$$

Explicit expressions will be given soon. All tensors are antisymmetric and antiselfdual in the index pairs $a, b$ and $c, d$. The tensors $C_{a b j k}$ and $D_{a b j}^{c d i}$ are symmetric and antihermitean-traceless in the $S U(2)$ indices, respectively. All five terms have to vanish independently.

The first condition $A_{a b}^{c}=0$ gives an equation for the covariant derivative of the auxiliary tensor $T_{a b}^{-}$:

$$
\mathcal{D}_{c} T_{a b}^{-}=i e^{\mathcal{K}}\left(\bar{X}^{J} \mathcal{D}_{d} F_{J}-\bar{F}_{J} \mathcal{D}_{d} X^{J}\right)\left(\delta_{c}^{d} T_{a b}^{-}-2 \delta_{[a}^{d} T_{b] c}^{-}+2 \eta_{c[a} T_{b]}^{-d}\right) .
$$

From $E_{a b}=0$ we get:

$$
T_{a}^{-d} \mathcal{F}_{d b}^{-}-T_{b}^{-d} \mathcal{F}_{d a}^{-}=0 .
$$

Next we have $D_{a b j}^{c d i}=0$ which after a few manipulations gives

$$
R(V) a{ }_{a b}{ }^{i}{ }^{2}=0 .
$$

Then we have $B_{a b}^{c d}=0$. With some effort and using 4.20 ) this can be brought to the form

$$
\mathcal{R}(M)_{a b}^{-c d}=\frac{-i}{16} T^{-e f} \mathcal{F}_{e f}^{-}\left(\delta_{[a}^{c} \delta_{b]}^{d}-\frac{1}{2} \varepsilon_{a b}^{c d}\right)+\frac{i}{8}\left[T_{a b}^{-} \mathcal{F}^{-c d}+T^{-c d} \mathcal{F}_{a b}^{-}\right] .
$$

The expression $\mathcal{R}(M)_{a b}^{-c d}$ is related to the modified Lorentz field strength $\mathcal{R}(M)_{a b}{ }^{c d}$ defined in (3.37) by antiselfdual projection in both pairs of indices. The terms on the right hand side are manifestly antiselfdual in both $a, b$ and $c, d$.

We can get more explicit information out of this tensor equation by making contractions. First we substitute the explicit form (3.31) of the $K$-connection into the definition (3.37) of $\mathcal{R}(M)_{a b}{ }^{c d}$ and we define the tensor

$$
C_{a b}{ }^{c d}=R_{a b}{ }^{c d}-2 \delta_{[a}^{[c} R_{b]}^{d]}+\frac{1}{3} R \delta_{[a}^{[c} \delta_{b]}^{d]},
$$

which in the Poincaré frame, i.e. after gauge fixing the conformal symmetries, becomes the Weyl tensor. Plugging this into (3.37) we get:

$$
\mathcal{R}(M)_{a b}^{c d}=C_{a b}^{c d}+D \delta_{[a}^{[c} \delta_{b]}^{d]}+2 i \delta_{[a}^{[c} \widetilde{R}(A)_{b]}^{d]} .
$$

Next we perform contractions that project onto the terms containing $R(A)_{a b}$ and $D$, respectively. First note that

$$
\varepsilon^{g b}{ }_{c d} \mathcal{R}(M)_{a b}{ }^{c d}=2 i R(A)^{g}{ }_{a}=2 \widetilde{R}(D)^{g}{ }_{a} .
$$

The first equations follows by computing the contraction, the second one is a consequence of the Bianchi identity (3.35). Performing the same contraction on the right hand side of (4.22) one gets zero and therefore we have

$$
R(A)_{a b}=0=R(D)_{a b} .
$$

Next we consider the trace part $\mathcal{R}(M)_{a b}{ }^{a b}$ to get information about $D$, using that the Weyltensor is traceless. This gives

$$
D=-\frac{i}{24} T^{-a b} \mathcal{F}_{a b}^{-}
$$

and plugging the result back we find

$$
\mathcal{R}(M)_{a b}^{-c d}=C_{a b}^{-c d}-\frac{i}{48} T^{-e f} \mathcal{F}_{e f}^{-}\left(\delta_{[a}^{c} \delta_{b]}^{d}-\frac{1}{2} \varepsilon_{a b}{ }^{c d}\right)
$$


(note that going from $\mathcal{R}(M)_{a b}{ }^{c d}$ to $\mathcal{R}(M)_{a b}^{-}{ }^{c d}$ involves the antiselfdual projection) and by (4.22) we find that the Weyl tensor is

$$
C_{a b}^{-c d}=\frac{-i}{24} T^{-e f} \mathcal{F}_{e f}^{-}\left(\delta_{[a}^{c} \delta_{b]}^{d}-\frac{1}{2} \varepsilon_{a b}^{c d}\right)+\frac{i}{8}\left[T_{a b}^{-} \mathcal{F}^{-c d}+T^{-c d} \mathcal{F}_{a b}^{-}\right]
$$

For generic antiselfdual tensors the right hand side has all algebraic symmetries of the Weyl tensor. But if we impose in addition spherical symmetry on this equation and look at the equation component by component, we find that it can only be satisfied trivially. To see this we first note that the Weyl tensor of the metric (4.1) has only one independent non-vanishing component:

$$
C_{01}^{01}=C_{23}^{23}=-2 C_{02}^{02}=-2 C_{03}^{03}=-2 C_{12}^{12}=-2 C_{13}^{13},
$$

whereas all other independent components vanish. In order to use this in equation (4.29) one has to project onto the antiselfdual part in both pairs of indices. This gives

$$
C_{01}^{-}{ }^{01}=\frac{1}{2} C_{01}{ }^{01}, C_{02}^{-02}=-\frac{1}{4} C_{01}{ }^{01}, \ldots
$$

On the right hand side one uses that an antiselfdual antisymmetric tensor only has one independent component $T_{01}^{-}=i T_{23}^{-}$. Evaluation (4.29) for the two components of the antiselfdual Weyl tensor given above implies that either $T_{01}^{-}=0$ or $\mathcal{F}_{01}^{-}=0$ and therefore

$$
C_{a b}{ }^{c d}=0 \text { and } T_{a b}^{-} \mathcal{F}^{-c d}=0 .
$$

By (4.27) this also implies

$$
D=0 \text {. }
$$

Finally $C_{a b j k}=0$ implies

$$
T_{a b}^{-} e^{\mathcal{K}}\left[\left(\bar{F}_{I}-\bar{X}^{J} F_{J I}\right) Y_{j k}^{I}-\bar{X}^{I} F_{I \widehat{A}} \widehat{B}_{j k}\right]=0 .
$$

We will see in the following sections that $T_{a b}^{-}=0$ leads to flat space. Now we take it to be non-vanishing and use that $F(X, \widehat{A})$ is an arbitrary function. Combining this with (4.17) implies

$$
Y_{i j}^{I}=0=\widehat{B}_{i j} .
$$

\subsubsection{Variation of the Spinor of the Second Compensating Multiplet}

The inclusion of the second compensating multiplet is not only needed for the consistency of the whole construction, but it also provides additional information for our problem. We take the non-linear multiplet as the second compensator. The S-invariant variation of its spinor yields

$$
\mathcal{F}_{a b}^{-}=0
$$

and

$$
A_{a}+\mathcal{A}_{a}=0
$$

The first equation implies

$$
\mathcal{F}_{a b}^{-I}=\widehat{F}_{a b}^{-I}=\mathcal{R}(M)_{a b}^{-c d}=0,
$$

using (4.13), (4.16) and (4.22). Since $\mathcal{A}_{a}$ as defined in (4.10) equals the Kähler connection (3.173) up to sign, we recognize that (4.37) is the equation of motion for the $U(1)$ gauge field.

Using the relation between $A_{a}$ and $\mathcal{A}_{a}$ we can rewrite the equations (4.11), (4.14) and (4.15) as

$$
\mathcal{D}_{a}\left(e^{\mathcal{K} / 2} X^{I}\right)=\mathcal{D}_{a}\left(e^{\mathcal{K}} / 2 F_{I}\right)=\mathcal{D}_{a}\left(e^{w \mathcal{K} / 2} \widehat{A}\right)=0 .
$$

As a consequence of (4.12) the $U(1)$ connection is flat. Now we pick a gauge where $A_{a}=0$ and go to the Poincaré frame by imposing the $K$ - and $D$-gauge,

$$
b_{\mu}=0 \text { and } e^{-\mathcal{K}}=m_{\text {Planck }}^{2} .
$$


Since the fields $X^{I}, F_{J}$ and $\widehat{A}$ are Lorentz scalars we are left with

$$
\partial_{a} X^{I}=\partial_{a} F_{I}=\partial_{a} \widehat{A}
$$

implying that the scalars $X^{I}$ and the background field $\widehat{A}$ are constant. Constancy of $X^{I}$ and $F_{J}$ implies $\mathcal{D}_{c} T_{a b}^{i}=0$ by (4.19). When we go to the Poincaré frame and impose the gauge $A_{a}=0$ as above then the covariant derivative still contains the spin connection because $T_{a b}^{-}$ is a Lorentz tensor. But using the explicit form of the spin connection for a static spherically symmetric metric A.22 one can verify that $T_{a b}^{-}$is actually constant, $\partial_{c} T_{a b}^{-}=0$.

\subsubsection{Variation of the Derivative of the Spinor $\zeta_{i}$}

Since all fermionic quantities have to vanish in our $\mathcal{N}=2$ invariant background, derivatives of spinors must have a vanishing variation. Usually this does not lead to new conditions, but in our case it does. When imposing that the $Q$-variation of $D_{a} \zeta_{i}$ vanishes up to a uniform S-transformation one gets an equation for the $K$-connection:

$$
f_{a b}-\frac{1}{2} \mathcal{D}_{a} \mathcal{D}_{b} \mathcal{K}-\frac{1}{4} \mathcal{D}_{a} \mathcal{K} \mathcal{D}_{b} \mathcal{K}+\frac{1}{8} \delta_{a b} \mathcal{D}_{c} \mathcal{K} \mathcal{D}^{c} \mathcal{K}=0
$$

In the Poincaré frame, when imposing the $K$ - and $D$-gauge conditions, we have

$$
\mathcal{D}_{a} \mathcal{K}=\partial_{a} \mathcal{K}=0
$$

and therefore the $K$-gauge field vanishes

$$
f_{a b}=0 \text {. }
$$

This yields an important information about the geometry of the background. Using the explicit form (3.31) of the composite $K$-gauge field we find

$$
R_{a b}=\frac{1}{8} T_{a}^{i j c} T_{c b i j}=\frac{1}{16} T_{a}^{-c} T_{c b}^{+}
$$

for the Ricci tensor and the corresponding Ricci scalar vanishes

$$
R=\frac{1}{16} T_{a}^{-c} T_{c a}^{+}=0
$$

by an identity which is generally valid for (anti-)selfdual tensors (see A.13). The Riemann tensor takes the same form as in the case of the near horizon geometry of an extremal ReissnerNordstrøm black hole: The Weyl tensor and Ricci scalar vanish while the traceless part of the Ricci tensor is non-trivial. Since the Ricci tensor is given in terms of the auxiliary tensor $T_{a b}^{-}$ we have to find the explicit form of this field.

\subsubsection{The Relation between the Auxiliary T-Field and the Gauge Fields}

The auxiliary $T$-field is related to the gauge fields. First recall that the dual gauge field $G_{\text {Iab }}^{-}$ is given by (3.81, 3.82)

$$
G_{I a b}^{-}=F_{I J} F_{a b}^{-J}+\frac{1}{4}\left(\bar{F}_{I}-F_{I J} \bar{X}^{J}\right) T_{a b}^{-}+\widehat{F}_{a b}^{-} F_{I \widehat{A}} .
$$

Now we contract this with $X^{I}$, use the homogenity of $F(X, \widehat{A})$ amd formula (4.38) to derive

$$
T_{a b}^{-}=4 i e^{\mathcal{K}}\left(F_{I} F_{a b}^{-I}-X^{I} G_{I a b}^{-}\right) .
$$

Since the definition of $G_{I a b}^{-}$involves $T_{a b}^{-}$, this is not an explicit expression for the $T$-field. 


\subsubsection{The Gauge Field Equations of Motion}

The gauge field equations of motion and the Bianchi identities have the following form:

$$
\begin{aligned}
\mathcal{D}^{\mu}\left(G_{I \mu \nu}^{-}-G_{I \mu \nu}^{+}\right) & =0, \\
\mathcal{D}^{\mu}\left(F_{\mu \nu}^{I-}-F_{\mu \nu}^{I+}\right) & =0 .
\end{aligned}
$$

They can be derived from the Lagrangian, as discussed in chapter 3 and appendix B. Alternatively these equations can be derived by setting the $Q$-variation of the covariant derivatives of the gaugini to zero (modulo the usual uniform $S$-transformation). This illustrates that full supersymmetry implies the equations of motion.

We can solve the equations of motion in a spherically symmetric and static background. Note that the field strengthes $F_{a b}^{I}$ and $G_{I a b}$ are not independent. When solving the equations we have to pick a set of independent components. Taking $F_{a b}^{I}$ (or $G^{I a b}$ ) as independent is inconvenient because then one of the two equations is very complicated. Instead on takes $F_{23}^{I}$ and $G_{I 23}$. Then all the equations take the form of Bianchi identities and can be solved as discussed in appendix $B$. For the spherically symmetric case one finds:

$$
\begin{aligned}
G_{I 01}^{-}-G_{I 01}^{+} & =i G_{I 23}=i \frac{e^{-2 f(r)}}{r^{2}} q_{I}, \\
F_{01}^{I-}-F_{01}^{I+}=i F_{23}^{I} & =i \frac{e^{-2 f(r)}}{r^{2}} p^{I},
\end{aligned}
$$

(using flat indices) with constants $p^{I}, q_{I}$, which, according to our definition (3.101) are the magnetic and electric charges.

Now we use that $\mathcal{F}_{a b}^{+}=0$ implies

$$
F_{I} F_{a b}^{I+}-X^{I} G_{I a b}^{+}=0
$$

to rewrite the expression for $T$

$$
T_{a b}^{-}=4 i e^{\mathcal{K}}\left(F_{I} F_{a b}^{I-}-X^{I} G_{I a b}^{-}\right)=4 i e^{\mathcal{K}}\left(F_{I} F_{a b}^{I}-X^{I} G_{I a b}\right) .
$$

Next we can use our solution for the gauge fields to express the $T$-field as

$$
T_{01}^{-}=i T_{23}^{-}=-4 e^{\mathcal{K} / 2} Z \frac{e^{-2 f(r)}}{r^{2}}
$$

where we defined

$$
Z=e^{\mathcal{K} / 2}\left(p^{I} F_{I}-q_{I} X^{I}\right) .
$$

The symbol $Z$ was chosen because this quantity resembles the central charge. Note however that $Z$ as defined here is not a number but a field. We will see later that in an asymptotically flat geometry the value of $Z$ at infinity is the central charge as defined in (3.148).

We already argued that in a supersymmetric, static and spherically symmetric background the quantities $X^{I}, F_{J}, Z, \mathcal{K}, T_{a b}^{-}$are constant in the Poincaré frame. This implies that $r^{2} e^{2 f(r)}$ must be constant and is given by

$$
r^{2} e^{2 f(r)}=-4 e^{\mathcal{K} / 2} \frac{Z}{T_{01}^{-}}
$$

so that we have fixed one of the unknown functions in (4.1). Obviously the constants on the right hand side must be related such that a real positive number results. In order to find this relation we now turn to a detailed investigation of the metric. 


\subsubsection{The Metric}

In order to proceed systematically we start by computing the curvature components of a spherically symmetric, static metric (4.1). The non-vanishing components of the Ricci tensor, with tangent space indices $a, b=0, \ldots, 3$ and the Ricci scalar are

$$
\begin{aligned}
R_{0}^{0} & =\left[g^{\prime \prime}+g^{\prime}\left(g^{\prime}+f^{\prime}\right)+\frac{2}{r} g^{\prime}\right] \mathrm{e}^{-2 f} \\
R_{1}^{1} & =\left[2 f^{\prime \prime}+g^{\prime \prime}+g^{\prime}\left(g^{\prime}-f^{\prime}\right)+\frac{2}{r} f^{\prime}\right] \mathrm{e}^{-2 f} \\
R_{2}^{2} & =R_{3}^{3}=\left[f^{\prime \prime}+f^{\prime}\left(g^{\prime}+f^{\prime}\right)+\frac{1}{r}\left(3 f^{\prime}+g^{\prime}\right)\right] \mathrm{e}^{-2 f}, \\
R & =\left[2\left(2 f^{\prime \prime}+g^{\prime \prime}\right)+2\left(f^{\prime 2}+g^{\prime 2}+f^{\prime} g^{\prime}\right)+\frac{4}{r}\left(2 f^{\prime}+g^{\prime}\right)\right] e^{-2 f}
\end{aligned}
$$

and the components of the Weyl tensor (again with tangent space indices) are equal to

$$
\begin{aligned}
C_{01}^{01}= & C_{23}^{23}=-2 C_{02}^{02}=-2 C_{03}^{03}=-2 C_{12}^{12}=-2 C_{13}^{13}= \\
& \frac{1}{3}\left[-f^{\prime \prime}+g^{\prime \prime}+\left(g^{\prime}-f^{\prime}\right)^{2}+\frac{1}{r}\left(f^{\prime}-g^{\prime}\right)\right] e^{-2 f} .
\end{aligned}
$$

We saw that full $\mathcal{N}=2$ supersymmetry implies

$$
R_{b}^{a}=-\frac{1}{16} T^{-a c} T_{b c}^{+} \text {and } C_{c d}^{a b}=0 .
$$

Moreover $T_{a b}^{-}$is constant and has only one independent non-vanishing component. Therefore all nonvanishing components of the Ricci tensor are equal up to sign

$$
R_{0}^{0}=R_{1}^{1}=-R_{2}^{2}=-R_{3}^{3}=\frac{1}{16}\left|T_{01}^{-}\right|^{2}=\text { constant } .
$$

Using (4.56) and the equations $R_{0}^{0}=R_{1}^{1}$ and $R_{0}^{0}=-R_{2}^{2}$ we get

$$
\begin{aligned}
f^{\prime \prime}-f^{\prime} g^{\prime}+\frac{1}{r}\left(f^{\prime}-g^{\prime}\right) & =0, \\
f^{\prime \prime}+g^{\prime \prime}+\left(f^{\prime}+g^{\prime}\right)^{2}+\frac{3}{r}\left(f^{\prime}+g^{\prime}\right) & =0 .
\end{aligned}
$$

In addition Weyl flatness implies

$$
f^{\prime \prime}-g^{\prime \prime}-\left(f^{\prime}-g^{\prime}\right)^{2}-\frac{1}{r}\left(f^{\prime}-g^{\prime}\right)=0 .
$$

Finally we have one inhomogenous equation, $R_{0}^{0}=$ constant. Using the other equations, it can be brought to the form

$$
\left(f^{\prime}\left(f^{\prime}-g^{\prime}\right)+\frac{1}{r}\left(2 f^{\prime}-g^{\prime}\right)\right) e^{-2 f}=\text { constant } .
$$

There is one linear combination of the three homogenous equations such that the second derivatives drop out:

$$
f^{\prime} g^{\prime}+\frac{1}{r} g^{\prime}=0
$$

Thus either $g^{\prime}=0$ or $f^{\prime}=\frac{-1}{r}$. The first case yields flat space. In the second case we have

$$
e^{f}=\frac{c}{r} .
$$

This is the same result (4.55) that we got from the gauge field equations, where the constant took the value $c=\sqrt{-4 e^{\mathcal{K} / 2} \frac{Z}{T_{01}^{-}}}$. We will check that this value of $c$ is consistent with what we get by solving the conditions on the Ricci and Weyl tensor. 
We proceed by using that the Weyl flatness condition implies

$$
e^{g-f}=A r^{2}+B .
$$

Defining $a=c A$ and $b=c B$ we find the metric

$$
d s^{2}=-\left(a r+\frac{b}{r}\right)^{2} d t^{2}+\frac{c^{2}}{r^{2}}\left(d r^{2}+r^{2} d \Omega^{2}\right) .
$$

Space-time factorizes into a sphere parametrized by $(\phi, \theta)$ times another two-surface parametrized by $(t, r)$. One can check that the Ricci scalar vanishes and that the Ricci tensor (with flat indices) is constant and only depends on $c$ whereas the coefficients $a, b$ drop out. This suggests that the constants $a, b$ do not have an invariant meaning, but that they can be changed by coordinate transformations, whereas $c$ has an invariant meaning and parametrizes a family of inequivalent (non-isometric) metrics. In order to prove this we have computed the Killing vectors associated with the $(t, r)$ surface. The result is that for all admissible choices (we have to request $a \neq 0$ or $b \neq 0$ to exclude singular cases and we have $c \neq 0$ ) three Killing vectors exist, and that the isometries satisfy the Lie algebra $s l(2, \mathbb{R}) \simeq s o(2,1)$. In view of the signature of the $(t, r)$ surface this leaves us with the unique possiblity of two-dimensional anti de Sitter space $A d S^{2} \simeq S O(2,1) / S O(1,1) \simeq S l(2, \mathbb{R}) / U(1)$. The full metric is the Bertotti-Robinson geometry $A d S^{2} \times S^{2}$. The standard parametrization is obtained by setting $b=0$ and rescaling $t$ such that $a=c^{-1}$ :

$$
d s^{2}=-\frac{r^{2}}{c^{2}} d t^{2}+c^{2} \frac{d r^{2}}{r^{2}}+c^{2}\left(\sin ^{2} \theta d \phi^{2}+d \theta^{2}\right) .
$$

It is possible to find the explicit coordinate transformation that eliminates $b$, but the expression is rather complicated and so we do not write it down. The constant $c$ specifies the radius of the geometry. We have found two expressions for the constant, one from the Ricci tensor and one from the gauge field equations. The formula for the Ricci tensor implies

$$
e^{2 g(r)}=e^{-2 f(r)}=\frac{r^{2}}{c^{2}}=\frac{1}{16}\left|T_{01}^{-}\right|^{2} r^{2},
$$

whereas the gauge field equations gave us

$$
e^{-2 f(r)}=-\frac{1}{4} e^{-\mathcal{K} / 2} T_{01}^{-} Z^{-1} r^{2} .
$$

This implies the relation

$$
T_{01}^{-}=-4 e^{-\mathcal{K} / 2} \bar{Z}^{-1}
$$

Thus the two constants $T_{01}^{-}, Z$ are related ${ }^{2}$ and one can express the radius either in terms of $T_{a b}^{-}$or in terms of $|Z|$ :

$$
e^{2 g(r)}=e^{-2 f(r)}=\frac{r^{2}}{c^{2}}=e^{-\mathcal{K}} \frac{r^{2}}{|Z|^{2}} .
$$

The later form is familiar from the case without chiral background. $Z$ is related to the $\mathcal{N}=2$ central charge. Therefore it is convenient to express all the other constants in terms of $Z$.

Our result describes the near horizon geometry of a static extremal black hole in Poincaré supergravity. The global form of the solution is (4.1) with two functions $f_{\mathrm{BH}}(r), g_{\mathrm{BH}}(r)$. In the near horizon region $r \rightarrow 0$ the black hole geometry approaches the $A d S^{2} \times S^{2}$ solution (4.72)

$$
\left(r^{2} e^{2 f(r)} \mathrm{BH}\right) \rightarrow_{r \rightarrow 0} r^{2} e^{2 f(r)}=e^{\mathcal{K}}|Z|^{2} \text { and } f_{\mathrm{BH}}(r) \simeq-g_{\mathrm{BH}}(r) .
$$

\footnotetext{
${ }^{2}$ We note in passing that the phase relation between $T_{01}^{-}$and $Z$ is such that the right hand side of 4.70 ) is positive, and that the constant $c$, when computed from the gauge field equation as $c=\sqrt{-4 e^{\mathcal{K} / 2} \frac{Z}{T_{01}^{-}}}$is real, $c=|Z|$
} 
Therefore the size of the event horizon is $A=4 \pi|Z|^{2}$. In order to describe $R^{2}$-corrections we identify the chiral background multiplet with the Weyl multiplet. The background scalar is

$$
\widehat{A}=T_{a b}^{i j} T^{k l a b} \varepsilon_{i k} \varepsilon_{j l}=-64 e^{-\mathcal{K} \bar{Z}^{-2}}
$$

In conclusion we have now fully specified the near horizon solution in terms of the field $Z$, which depends on the charges and on the scalar fields. In the Bertotti-Robinson geometry $Z$ is constant, but in the full black hole solution, which only approaches the Bertotti-Robinson geometry asymptotically, it is in general an $r$-dependent quantity through the $r$-dependence of the scalar fields.

We conclude this lengthy section by collecting the formulae which describe a static and spherically symmetric $\mathcal{N}=2$ vacuum and, simultanously, the near horizon geometry of a BPS black hole. The metric, gauge fields and scalars are

$$
\begin{aligned}
& d s^{2}=-e^{-2 f(r)} d t^{2}+e^{2 f(r)}\left(d r^{2}+r^{2} d \Omega^{2}\right) \\
& G_{I 23}=\frac{e^{-2 f(r)}}{r^{2}} q_{I}, \quad F_{23}^{I}=\frac{e^{-2 f(r)}}{r^{2}} p^{I}, \quad X^{I}=\text { const. }
\end{aligned}
$$

where the function $f(r)$ is related to the field $Z=p^{I} F_{I}(X, \widehat{A})-q_{I} X^{I}$ by

$$
e^{2 f(r)} r^{2}=e^{\mathcal{K}}|Z|^{2}
$$

The field $e^{\mathcal{K}}$ is the compensator for dilation invariance and becomes a constant in the Poincaré frame. The values of the auxiliary $T$-field and of the background scalar $\widehat{A}$ are given in (4.71) and (4.74) in terms of $Z$. When we substitute the expression for $\widehat{A}$ into the definition of $Z$ and use the homogenity of $F_{I}(X, \widehat{A})$ we get an equation for $|Z|^{2}$ in terms of the $X^{I}$, whereas the phase of $Z$ remains arbitrary (see also the beginning of chapter 6). We will see in the next section that the $X^{I}$ and therefore $|Z|^{2}$ and the complete solution can be expressed in terms of the electric and magnetic charges.

\subsection{The Entropy}

\subsubsection{The Entropy Formula}

With the Poincaré frame action ((3.111), subject to gauge conditons) and the near horizon solution at our disposal we can now use Wald's formula to compute the entropy. First remember that the entropy is given by (2.5.5)

$$
\mathcal{S}=2 \pi \oint d^{2} x \sqrt{h} \varepsilon_{a b} \varepsilon_{c d} \frac{\partial \mathcal{L}_{\text {Poinc }}}{\partial R_{a b c d}}
$$

where the integral is over the event horizon, $h$ is the absolute value of the determinant of the pulled-back metric and $\varepsilon_{a b}$ is the binormal. We have already normalized the Poincaré action such that we can use the conventions of chapter 2. First we have to compute the partial derivative with respect to the Riemann tensor. To do so we have to remind ourselves that the components of the chiral background field are related to the Weyl multiplet and therefore a lot of the terms depend on the Riemann tensor. However there are no derivatives of the Riemann tensor present so that the version (2.55) of the entropy formula applies. The result is:

$$
\frac{\partial \mathcal{L}_{\text {Poinc }}}{\partial R_{a b c d}}=-\frac{1}{2 \kappa^{2}} \eta^{a c} \eta^{b d}+\frac{i}{16 \pi}\left(F_{\widehat{A} I} \mathcal{F}_{e f}^{I-} \frac{\partial \widehat{F}_{e f}^{-}}{\partial R_{a b c d}}+F_{\widehat{A} \widehat{A}} \widehat{F}_{e f}^{-} \frac{\partial \widehat{F}_{e f}^{-}}{\partial R_{a b c d}}+F_{\widehat{A}} \frac{\partial \widehat{C}}{\partial R_{a b c d}}-h . c .\right)
$$

The first term comes from the Einstein-Hilbert term and gives the standard Bekenstein-Hawking entropy. The other terms are deviations from the area law that result from the additional curvature terms. Using that for our solution $\mathcal{F}_{a b}^{-I}=\widehat{F}_{a b}=0$ the formula simplifies:

$$
\frac{\partial \mathcal{L}_{\text {Poinc }}}{\partial R_{a b c d}}=-\frac{1}{2 \kappa^{2}} \eta^{a c} \eta^{b d}+\frac{i}{16 \pi}\left(F_{A} \frac{\partial \widehat{C}}{\partial R_{a b c d}}-\text { h.c. }\right)
$$


Now remind the transformation rules $(3.99)$ of the function $F(X, \widehat{A})$ and of its derivatives. Most of these quantities transform in a complicated, non-covariant way under symplectic transformation. This is true in particular for $F_{\widehat{A} I}$ and $F_{\widehat{A} \widehat{A}}$, but luckily these quantities do not contribute because there coefficients vanish. The remaining correction term involves $F_{\widehat{A}}$ which is a symplectic function.

It remains to compute $\frac{\partial \widehat{C}}{\partial R_{a b c d}}$. We now use that in a bosonic background

$$
D_{a}\left(D^{c} T_{c b i j}\right)=\mathcal{D}_{a}\left(\mathcal{D}^{c} T_{c b i j}\right)-f_{a}{ }^{c} T_{c b i j},
$$

to rewrite (the bosonic terms in) $\widehat{C}$ as ${ }^{5}$

$$
\begin{aligned}
\widehat{C} & =-8 T^{a b-}\left\{\mathcal{D}_{a}, \mathcal{D}^{c}\right\} T_{c b}^{+}+16 T^{a b-} f_{a}{ }^{c} T_{c b}++64 \mathcal{R}(M)_{c d}^{-a b} \mathcal{R}(M)_{c d}^{-a b} \\
& +32 R(V)_{a b k}^{-l} R(V)_{a b l}^{-k} .
\end{aligned}
$$

We compute

$$
\frac{\partial \widehat{C}}{\partial R_{a b}{ }^{c d}}=8 T^{a f-} T_{c f}+\delta_{d}^{b}+128 \mathcal{R}(M)^{-m n}{ }_{l q} \frac{\partial \mathcal{R}(M)_{m n}^{-l q}}{\partial R_{a b}{ }^{c d}} .
$$

Using that $\mathcal{R}(M)_{a b}^{-c d}=0$ on the horizon we find

$$
\epsilon_{a b} \epsilon^{c d} \frac{\partial \widehat{C}}{\partial R_{a b}{ }^{c d}}=16 T^{01+} T^{01-} .
$$

Performing the integral over the horizon and expressing everything in terms of $Z$ we finally get our model-independent entropy formula

$$
\mathcal{S}=\pi\left(G_{N}^{-1}|Z|^{2}-256 \operatorname{Im} F_{\widehat{A}}(X, \widehat{A})\right), \quad \text { where } \widehat{A}=-64 \bar{Z}^{-2} e^{-\mathcal{K}} .
$$

This is one of our central results [91]. Note that we expressed the gravitational coupling $\kappa$ by Newtons constant. The dilatational gauge fixing relates $e^{-\mathcal{K}}$ to the Planck scale and therefore to $G_{N}: e^{-\mathcal{K}}=m_{\text {Planck }}^{2}=G_{N}^{-1}=8 \pi \kappa^{-2}$. The formula is manifestly convariant with respect to symplectic transformations.

In view of the complications we had to go through this is a strikingly simple result. All modifications through additional curvature terms are captured by the function $F_{\widehat{A}}$, and we did not need to specify this function so that the formula is completely model-independent. Now recall that $Z$ is a function of the $X^{I}$ and of the charges $p^{I}, q_{I}$ and that the fields $X^{I}$, which themselves are functions of the unconstrained physical scalars. Therefore the entropy is a complicated function of the charges and of the scalars on the horizon. Normally one would expect that the scalars on the horizon can take arbitrary or at least a continuous range of values so that the entropy can vary continuously. Such a behaviour would clash with the intended interpretation of the entropy as the degeneracy of the macroscopic state of the black hole. The next step is to argue that the scalars cannot take arbitrary values on the horizon, but are themselves determined by the charges.

\subsubsection{The Stabilization Equations}

In absence of higher derivative terms a set of beautiful relations have been derived, which express the scalars on the event horizon, $z_{\text {hor }}^{A}=\left(X^{A} / X^{0}\right)_{\text {hor }}$ in terms of the charges $\left(p^{I}, q_{J}\right)$ 121, 122, 123, 124. These relations are called the stabilization equations and they take the form

$$
\left[\bar{Z}\left(\begin{array}{c}
X^{I} \\
F_{J}
\end{array}\right)-Z\left(\begin{array}{c}
\bar{X}^{I} \\
\bar{F}_{J}
\end{array}\right)\right]_{\mathrm{hor}}=i\left(\begin{array}{c}
p^{I} \\
q_{J}
\end{array}\right),
$$

\footnotetext{
${ }^{3}$ The commutator part of the double derivative vanishes, $T^{a b-}\left[\mathcal{D}_{a}, \mathcal{D}^{c}\right] T_{c b}^{+}=0$, due to (anti-)selfduality of $T_{a b}^{ \pm}$.

${ }^{4}$ We set $e^{\mathcal{K}}=1$ for the rest of this chapter.
} 
where $F(X)$ is the prepotential, $Z$ is the function defined in (4.54) and the expression on the left side is evaluated on the event horizon. The name stabilization equations is motiviated by the fact that in the presence of non-trivial scalar fields one needs to impose conditions on their near horizon behaviour in order to avoid that they take singular values on the horizon. In the context of string compactifications, where the scalar fields are moduli, such singularities can be understood as compactification artefacts. The values of the moduli specify the four-dimensional coupling and the geometry of the internal compact space. Singularities in these quantities do not signal a breakdown of the underlying microscopic higher-dimensional theory, but a breakdown of the effective four-dimensional description due to decompactification or strong four-dimensional coupling. Therefore the singularity can be removed by lifting the solution to a higher-dimensional solution of the fundamental theory. This can be explicitly studied in the case of toroidal compactifications. Conversely one also sees that the compactification of a regular higher dimensional solution often leads to a singular lower dimensional solution, unless suitable conditions are imposed which stabilize the moduli.[.

BPS black holes in $\mathcal{N}=2$ theories do not result from simple toroidal compactifications. But one can analyse the condition for having regular moduli using four-dimensional supergravity. The result is that on the horizon full $\mathcal{N}=2$ supersymmetry must be restored, implying that the solution interpolates between two vacua, Minkowski space and the Bertotti-Robinson geometry. Moreover this implies the stabilization equations (4.85).

The equations impose $2 N_{V}+2$ real conditions on $2 N_{V}+2$ complex quantities $X^{I}, F_{J}$. Since the $F_{J}$ are functions of the $X^{I}$ only half of these quantities are independent, and generically these equations will fix the moduli $z_{\text {hor }}^{A}$ at the horizon in terms of charges $\left(p^{I}, q_{J}\right)$. If the prepotential is sufficiently simple one can solve the equations explicitly. We will see examples of this when discussing concrete models in chapter 6. The behaviour of the moduli in the asymptotically flat region at infinity, $z_{\infty}^{A}=\left(X^{A} / X^{0}\right)_{\infty}$, is completely different in that the values of the moduli are not fixed, but can be chosen arbitrarily. The scalar equations of motion in a static spherically symmetric BPS background can be interpreted as a dynamical system with the radius $r$ as 'time'. They describe the evolution of the moduli as functions of $r$ from their arbitrary values at infinity to their fixed values at the horizon. Thus the dynamical system exhibits fixed point behaviour. Since the fixed points are attractive, they form an attractor and the mechanism has been called the supersymmetric attractor mechanism.

The asymptotic form of the metric at the horizon is

$$
d s^{2}=-\frac{r^{2}}{|Z|^{2}} d t^{2}+\frac{|Z|^{2}}{r^{2}} d \vec{x}^{2},
$$

and therefore the Bekenstein-Hawking entropy is given by

$$
\mathcal{S}=\frac{A}{4}=\pi|Z|_{\text {hor }}^{2},
$$

where $|Z|_{\text {hor }}$ is the value of $|Z|$ on the horizon. Since on the horizon the scalar fields are fixed in terms of the charges, it follows that $|Z|_{\text {hor }}$ and the entropy are exclusively determined by the charges.

The stabilization equations can be reformulated as an extremalization condition on $|Z|$, evaluated on the horizon. In fact it was first directly observed that for static supersymmetric backgrounds the dynamical system governing the $r$ evolution of the function $f(r)$ shows attractive fixed point behaviour [121. Later it was shown that the fixed points are determined by the stationary points of $|Z|$ as a function of the moduli, keeping the charges fixed, by

$$
\partial_{A}\left|Z\left(z, \bar{z}, p^{I}, q_{J}\right)\right|=0,
$$

where $\partial_{A}$ is the partial derivative with respect to the scalars $z^{A}$ [123, 124]. It was also shown that this is equivalent to the stabilization equations (4.85). In practice it is much easier to solve

\footnotetext{
${ }^{5}$ From now on we will call scalars with flat potentials moduli, even when not explicitly referring to a string compactification.
} 
the stabilization equations than to perform the explicit extremization 131. This is the reason why we started our discussion with them. Note also that they can be derived directly from the Killing spinor equations, without first deriving the equivalent statement (4.88) [126, 127, 128].

It turns out that $|Z|$ is not just stationary at the fixed point but in fact takes its minimum, which motivates the term minimal area principle 132|. It is known from explicit examples that several minima with the same charges may exist 133, 134, 135. Thus in general every fixed point has a finite bassin of attraction and is characterized by an area code. It might also happen that the fixed point sits on the boundary of the moduli space. The global aspects of the attractor mechanism are not yet fully understood. We refer to [133] for a more complete discussion, which also describes the deep relations of the attractor mechanism to number theory and to the geometry of Calabi-Yau manifolds. We also note that the attractor mechanism applies generally to BPS solitons in extended supergravity. Besides four-dimensional and five-dimensional BPS black holes in $\mathcal{N}=2,4,8$ supergravity [123, 124, 136] this applies more generally to BPS $p$-branes 137. We refer to 138 for a review.

One can give yet another derivation of the stabilization equations, which is based on electricmagnetic duality, or symplectic covariance [131]. Given a black hole solution that in the near horizon area exhibits full $\mathcal{N}=2$ supersymmetry we know that the solution is described by two symplectic vectors, $\left(p^{I}, q_{J}\right)$ and $\left(X^{I}, F_{J}\right)$. Assuming that the solution is uniquely determined by the charges the two vectors must be related. The only possible relation admitted by symplectic invariance is that they are proportional. Since $\left(p^{I}, q_{J}\right)$ is real whereas $\left(X^{I}, F_{J}\right)$ is complex the constant of proportionality must be complex. Using the $D$-gauge condition this constant is fixed to be $Z$ and this way one obtains (4.85). This argument has the advantage that it can be directly applied to the case with $R^{2}$-terms without having to find the full interpolating solution. The only possibility which respects symplectic covariance is 125:

$$
\left[\bar{Z}\left(\begin{array}{c}
X^{I} \\
F_{J}(X, \widehat{A})
\end{array}\right)-Z\left(\begin{array}{c}
\bar{X}^{I} \\
\bar{F}_{J}(\bar{X}, \bar{A})
\end{array}\right)\right]_{\mathrm{hor}}=i\left(\begin{array}{c}
p^{I} \\
q_{J}
\end{array}\right) .
$$

This form of the stabilization equation will be used later to determine the black hole entropy in terms of the charges.

\subsection{Black hole Solutions}

So far we have restricted our attention to the near horizon geometry and to the black hole entropy. In this section we will give a short review of the results obtained in [126, 127, 128] on the full BPS black hole solution. These results were obtained using the on-shell formulation and refer to the minimal Lagrangian without $R^{2}$-terms. At the end we will briefly comment on the case with $R^{2}$-terms.

BPS solutions can be found by looking for a static (or stationary) and asymptotically flat geometry that is invariant under half of the $\mathcal{N}=2$ supersymmetries. This means that the supersymmetry variations of the fermions vanish in the bosonic background for specific choices of the $\epsilon_{i}$, where four of the eight real components are fixed in terms of the others. The resulting geometry has four Killing spinors rather then eight and is invariant under four of the eight supersymmetry transformations.

The analysis can be carried out without imposing spherical symmetry. metric that is static and has a conformally flat space part ('conformastatic')

$$
d s^{2}=-e^{2 g(\vec{x})} d t^{2}+e^{2 f(\vec{x})} d \vec{x}^{2}
$$

with two unknown functions. It was shown by Tod 129 that the most general static supersymmetric metric is of that type and that the two functions in the supersymmetric case must be related by $f=-g$.

\footnotetext{
${ }^{6}$ In our analysis of $\mathcal{N}=2$ supersymmetry we imposed spherical symmetry to simplify the problem. The generalization to multi-centered BPS solutions is currently under investigation [19].
} 
The projection which identifies the four unbroken supersymmetries can be found in various ways. One can start from the supersymmetry algebra and ask for a massive state at rest which saturates the BPS bound. This implies that the Bogomolnyi matrix, which is the matrix of all $Q$-anticommutators, evaluated in the background, must have a zero mode. This implies a condition on the asymptotic form of the Killing spinor, which expresses half of the components in terms of the others. Alternatively one can look at the $Q$-variations of the gravitini field strengthes. If one does not impose full $\mathcal{N}=2$ supersymmetry, then one obains a relation between the components of the Killing spinor, which fixes half of them. This is an integrability condition for the vanishing of the gravitini variations. One can also directly solve the gravitini variations without looking at the integrability condition, as for example done in 126, 127, 128 .

In the case of static (and stationary) BPS backgrounds the projection takes the form

$$
\epsilon_{i}=i \gamma_{0} \varepsilon_{i j} \epsilon^{j}
$$

(up to a phase factor that we will discuss later). Moreover the vanishing of the gaugino variations implies the so-called generalized stabilization equations 126 .

$$
i\left(X^{I}(z)-\bar{X}^{I}(\bar{z})\right)=H^{I}, \quad i\left(F_{J}(z)-\bar{F}_{J}(\bar{z})\right)=H_{J}
$$

where $\left(X^{I}(z), F_{J}(z)\right)$ is the holomorphic section. The functions $H^{I}(\vec{x}), H_{J}(\vec{x})$ are related to the magnetic parts of the gauge fields by

$$
F_{m n}^{I}=\frac{1}{2} \epsilon_{m n p} \partial_{p} H^{I}, \quad G_{J m n}=\frac{1}{2} \epsilon_{m n p} \partial_{p} H_{I},
$$

and therefore form a symplectic vector. Note that $m, n=x, y, z$ are spatial world indices and that the $\epsilon$-symbol is the standard real one, $\epsilon_{x y z}=1$. When imposing the gauge field equations of motion the functions $H^{I}, H_{J}$ must be harmonic. The generalized stabilization equations are the rationale behind the observation that the full black hole solution is obtained from the near horizon solution by replacing electric and magnetic charges with harmonic functions [139].

The static supersymmetric metric takes the form (4.90) with

$$
e^{-2 g}=e^{2 f}=i\left(\bar{X}^{I}(\bar{z}) F_{I}(z)-X^{I}(z) \bar{F}_{I}(\bar{z})\right)=e^{-K},
$$

where $K$ is the Kähler potential. As a further condition of unbroken supersymmetry in a static background one finds that the Kähler connection (3.173.3.174) has to vanish,

$$
A_{\mu}=-\frac{i}{2}\left(\partial_{A} K \partial_{\mu} z^{A}-\partial_{\bar{A}} K \partial_{\mu} \bar{z}^{\bar{A}}\right)=0 .
$$

Moreover the $Z$-field has to be real.

Depending on the choice of harmonic functions one can describe a single BPS black hole or a static ensemble of BPS black holes. The above analysis has even been generalized to stationary BPS backgrounds and to gravitational instantons. In these cases the Kähler connection is non-vanishing and determines the off-diagonal components $g_{t m}(\vec{x})$ of the metric. The resulting solutions describe rotating geometries, Eguchi-Hanson and Taub-NUT spaces [128].

We will consider only static solutions in the following. Let us first reformulate the above solution in order to bring it closer to the formalism we use in this paper. In particular we use the covariantly holomorphic section $\left(X^{I}, F_{J}\right)$ instead of the holomorphic one. We would also like to formulate the solution in a manifestly $U(1)$ and Kähler invariant way. In the above solution the metric is given in terms of the Kähler potential. As we discussed earlier, a general holomorphic reparametrization of the scalar manifold acts on the holomorphic section as a symplectic transformation accompanied by a Kähler transformation. The Kähler potential is symplectically invariant but is changed by the Kähler transformation. Since the function $e^{2 f}$ is invariant under reparametrizations of the scalar manifold it seems that the solution imposes a 'gauge fixing condition' that picks a particular class of parametrizations of the scalar manifold. A related point is that the projection (4.91) explicitly breaks chiral $U(1)$ invariance and thus 
fixes the phase of $Z$, such that $Z$ is real. One can, however, replace (4.91) by the $U(1)$ covariant version

$$
\epsilon_{i}=\gamma_{0} \frac{\bar{\Sigma}}{|\Sigma|} \varepsilon_{i j} \epsilon^{j}
$$

where $\Sigma$ is a field of $U(1)$ weight -1 , which is later on determined by the Killing spinor equations.

The calculation of [128 can now be recast in a $U(1)$ invariant way. Alternatively one can do the calculation in the off-shell formulation, which allows to include the Weyl background [19].

In both cases the Killing equations imply generalized stabilization equations of the form

$$
\bar{\Sigma}\left(\begin{array}{c}
X^{I} \\
F_{J}
\end{array}\right)-\Sigma\left(\begin{array}{c}
\bar{X}^{I} \\
\bar{F}_{J}
\end{array}\right)=i\left(\begin{array}{c}
H^{I} \\
H_{J}
\end{array}\right),
$$

with

$$
\Sigma=H^{I} F_{I}-H_{I} X^{I}
$$

The metric is given by

$$
e^{-2 g}=e^{2 f}=|\Sigma|^{2} .
$$

In addition one finds that the Kähler connection takes the form

$$
A_{r}=\frac{i}{2} \partial_{r} \log \frac{\Sigma}{\bar{\Sigma}}
$$

and that $\bar{\Sigma} Z$ has to be real.

Note that (4.99) is invariant under reparametrizations of the scalar manifold because the covariantly holomorphic section transforms by a symplectic transformation combined with a local $U(1)$ rotation, and $\Sigma$ is symplectically invariant while the phase is irrelevant for $|\Sigma|$. The original solution is recovered in a parametrization where $\Sigma=\bar{\Sigma}=-e^{-K / 2}$ : With this condition the generalized stabilization equations take the form (4.92), the metric is given in terms of the Kähler potential, the Kähler connection vanishes and the $Z$-field is real.

We now take a closer look onto static single-centered solutions, which are obtained by choosing the harmonic functions

$$
H^{I}=h^{I}+\frac{p^{I}}{r}, \quad H_{I}=h_{I}+\frac{q_{I}}{r} .
$$

First we analyse the behaviour near the horizon to make contact with the discussion of the last section. We find that

$$
(r \Sigma)_{\text {hor }}=Z_{\text {hor }}
$$

and therefore the generalized stabilization equations evaluated at the horizon are the stabilization equation (4.85).

The Bekenstein-Hawking entropy $\mathcal{S}=\frac{A}{4}$ can be extracted from the behaviour of the metric at the horizon,

$$
A=4 \pi\left(e^{2 f} r^{2}\right)_{r=0} .
$$

Using that

$$
\left(e^{2 f} r^{2}\right)_{r=0}=\left(|\Sigma|^{2} r^{2}\right)_{r=0}=|Z|_{r=0}^{2}=|Z|_{\text {hor }}^{2}
$$

we obtain

$$
\mathcal{S}=\pi|Z|_{\text {hor }}^{2},
$$

where $|Z|_{\text {hor }}$ only depends on the charges $p^{I}, q_{J}$ as a consequence of the stabilization equations.

Let us now study the asymptotic behaviour at infinity and compute the ADM-mass. The asymptotic value of $\Sigma$ is

$$
\Sigma_{\infty}=h^{I} F_{I}(\infty)-h_{I} X^{I}(\infty) .
$$

In order to have the correct normalization of the metric at infinity we need to impose

$$
e^{2 g(\infty)}=e^{2 f(\infty)}=|\Sigma|_{\infty}^{2}=1 .
$$


This yields one real condition on the parameters $h^{I}, h_{J}$, which characterize the behaviour of the harmonic functions at infinity. There is a second condition which results from the fact that the Kähler connection $A_{\mu}$ has to take the particular form (4.100) in a supersymmetric static background. This implies

$$
h^{I} q_{I}-h_{I} p^{I}=0 .
$$

Thus the family of solutions is parametrized by $h^{I}, h_{J}, p^{I}, q_{J}$ subject to two real constraints. The parameters $p^{I}, q_{J}$ are discrete by Dirac quantization, and independend because they are the electric and magnetic charges with respect to the $N_{V}+1$ gauge fields in the theory. The parameters $h^{I}, h_{J}$ are continuous and subject to 2 real constraints. This corresponds to the fact that the theory contains $N_{V}+1$ independent gauge fields, but only $N_{V}$ independent complex scalar fields. The scalars can take arbitrary (or at least a continuous range of) values at infinity. In other words the solution is parametrized by the charges $p^{I}, q_{J}$ and the moduli at infinity, $z_{\infty}^{A}$. The ADM mass is obtained by expanding the metric component $g_{t t}$ to lowest order in $\frac{1}{r}$ :

$$
-e^{2 g}=-\left(1-2 \frac{M_{A D M}}{r}+\mathcal{O}\left(\frac{1}{r^{2}}\right)\right) .
$$

Using the explicit form of $\Sigma$ one can show that

$$
M_{A D M}=\bar{\Sigma}_{\infty} Z_{\infty}
$$

Since $|\Sigma|_{\infty}=1$ we can rewrite the ADM mass:

$$
M_{A D M}=|Z|_{\infty}=\left|p^{I} F_{I}(\infty)-q_{I} X^{I}(\infty)\right|
$$

This is in fact the BPS mass formula

$$
M_{B P S}=|Z| \text {, }
$$

where $Z$ is the central charge defined in (3.148). To make this explicit one uses that the relation $F_{I} F_{a b}^{+I}-X^{I} G_{I a b}^{+}=0$ that we derived earlier for $\mathcal{N}=2$ vacua also holds for BPS configurations with four Killing spinors 128 . Thus one has

$$
T_{a b}^{-}=F_{I} F_{a b}^{I}-X^{I} G_{I a b}
$$

The asymptotic form of the gauge field is

$$
T_{23}^{-} \simeq_{r \rightarrow \infty} \frac{p^{I} F_{I}(\infty)-q_{I} X^{I}(\infty)}{r^{2}}=\frac{Z_{\infty}}{r^{2}}
$$

where we used the definition of $p^{I}, q_{J}$ in terms of $F_{a b}^{I}, G_{I a b}$. Then the central charge is

$$
Z=\frac{1}{4 \pi} \oint T^{-}=\frac{1}{4 \pi} \int_{S_{\infty}^{2}} T_{23}^{-} r^{2} d \Omega=Z_{\infty} .
$$

Therefore the asymptotic value $Z_{\infty}$ of the $Z$-field (4.54) is the central charge (3.148) carried by the BPS solution and we obtain the standard BPS mass formula, as expected. Obviously the mass depends both on the charges $p^{I}, q_{J}$ and on the moduli $z_{\infty}^{A}$.

For any given set of charges $p^{I}, q_{J}$ one can obtain a particularly simple solution by setting the scalar fields to constant values. Consistency then requires that these values are precisely the fixed point values that supersymmetry dictates, $z^{A}(r)=z_{\mathrm{hor}}^{A}=z_{\mathrm{FP}}^{A}\left(p^{I}, q_{J}\right)$. This implies that $Z_{\infty}=Z_{\text {hor }}$ and since $|Z|_{\text {hor }}$ takes the minimal value (as a function of the moduli $z^{A}$ ), it follows that the ADM mass is minimized as a function of the moduli $z_{\infty}^{A}$. It is of course plausible that for black hole solutions with scalar fields the minimum of the mass is obtained when the scalars

\footnotetext{
${ }^{7}$ Remember that the scalar manifold can be an arbitrary special Kähler manifold. The precise range of value of the scalar fields depends on how one parametrizes this manifold.
} 
are constant. Such black hole solutions have been called double extremal [140], motivated by the following set of inequalities:

$$
M_{B H}\left(z_{\infty}, p^{I}, q_{J}\right) \geq M_{B P S}\left(z_{\infty}, p^{I}, q_{J}\right) \geq M_{F P}\left(z_{\mathrm{FP}}\left(p^{I}, q_{J}\right), p^{I}, q_{J}\right) .
$$

This means that given a general (non-extremal) static black hole with charges $p^{I}, q_{J}$ and generic values of the moduli $z_{\infty}^{A}$ one can minimize the mass, while keeping charges and moduli fix, by going to the extremal limit, where the black hole becomes supersymmetric. Then one can further minimize the BPS mass over the moduli space, which yields the black hole with the minimal possible mass for the given set of charges. For constant scalars the relation between $\Sigma$ and $Z$ simplifies with the result

$$
e^{-2 g}=e^{2 f}=|\Sigma|^{2}=\left(1+\frac{|Z|}{r}\right)^{2} .
$$

Therefore the metric of a double extremal black hole is the extremal Reissner-Nordstrøm metric.

In the above discussion we did not consider $R^{2}$-terms but worked with the mininal Lagrangian. Given the form of the solution we expect that in presence of $R^{2}$-terms the solution is modified by replacing the prepotential $F(X)$ by the full function $F(X, \widehat{A})$. As is obvious from our discussion of $\mathcal{N}=2$ solutions in presence of $R^{2}$-terms it is, however, difficult and involved to actually prove this statement. We will not enter the investigation here, but refer to [19].

Finally we would like to point out that there are further interesting topics in relation to $D=4, \mathcal{N}=2$ black holes, which we did not touch in the short review given above. Extremal black holes in five-dimensional $\mathcal{N}=2$ supergravity ( 8 real supercharges) are very similar to their four-dimensional counterparts, because of the so-called very special geometry 141, which controls the vector multiplet couplings. This has been used to construct and analyse extremal black hole solutions [142, 143, 144. In the context of string or M-theory compactifications on Calabi-Yau threefolds black holes can be used as probes to investigate topological phase transitions 145, 128, 146, 133. One can use T-duality to map extremal black holes onto Euclidean wormholes, which are generalizations of the ten-dimensional type IIB D-instanton 147 . We refer to 138 for a nice review of BPS black holes in extended supergravity and to 148, 149 for a recent account on the role of so-called generating solutions for both macroscopic and microscopic aspects of BPS black holes in $N=8$ supergravity.

During the last year the metric on the moduli space of multiblack hole solutions has been studied for both five- and four-dimensional $\mathcal{N}=2$ supergravity coupled to vector multiplets 150. 151, 152. The extension of these results to the case with $R^{2}$ terms is currently under investigation 153. The dynamics of near coincident black hole is described by superconformal quantum mechanics on the moduli space, see [154] for a review.

\subsection{More recent results on stationary BPS solutions in presence of $R^{2}$-terms}

More recently we have succeeded in finding all stationary space-times with residual supersymmetry embedded according to (4.96), for an arbitrary function $F(X, \widehat{A})$. We refer the reader to [19] for the details and only summarize the most important results here. The most general stationary solution can be fully specified in terms of $2 N_{V}+2$ harmonic functions, subject to the generalized stabilization equations (4.97) with the prepotential $F(X)$ replaced by the full function $F\left(X^{I}, \widehat{A}\right)$, as conjectured above. We emphasize that the generalized stabilization equations are not only sufficient, but also necessary for having partial supersymmetry. A particular subclass of solutions describes single- and multi-centered configurations of extremal black holes and these solution approach the Bertotti-Robinson solution on their horizons. In [19] we included an arbitrary number of neutral hypermultiplets into the analysis and found that the hypermultiplet scalars have to be constant in stationary space-times with residual supersymmetry embedded according to (4.96). Thus including hypermultiplets does not lead to more general solutions. 
In 19 we also proved two further results about solutions with full $N=2$ supersymmetry (again for arbitrary $F(X, \widehat{A})$ ). The first is that the Bertotti-Robinson solution is the unique stationary $N=2$ solution. The properties of spherical symmetry and staticity, which we imposed separately in section 4.1 follow in fact automatically from full supersymmetry. The second new result is that in any stationary $N=2$ solution, except the limiting case of Minkowski space, the scalar fields are determined in terms of the electric and magnetic charges by the stabilization equations (4.89). In other words one does not need top invoke arguments based on the flow from a BPS solution to a fully supersymmetric configuration in order to derive (4.89). The stabilization equations are a necessary condition for having full $\mathcal{N}=2$ supersymmetry, and the scalars must take their fixed-point values, if non-trivial gauge fields are present. If there are no charges, one gets flat Minkowski space and the scalars can take arbitrary constant values.

What is the origin of this additional condition for $N=2$ solutions? As explained above, new conditions may arise when considering the variations of covariant derivatives of fermions. In [19] we succeeded in finding all such conditions, and it turned out that there is one additional condition, which finally implies the stabilization equations. In order to find all conditions coming from derivatives of fermions in a transparent and systematic way, it is advantagous to use a hypermultiplet rather than a non-linear multiplet as the second compensating multiplet. Therefore we do not enter into the details here, but refer the reader once again to [19]. 


\section{Chapter 5}

\section{Fourdimensional String and M-theory Compactifications}

In the last two chapters we discussed $\mathcal{N}=2$ supergravity and its black hole solutions. Since supergravity is not consistent as a quantum theory, it must be embedded into a larger consistent theory. The most promissing candidate is string theory, and in this chapter we review how fourdimensional $\mathcal{N}=2$ supergravity arises as the low energy effective theory of compactifications of ten-dimensional string theories and of eleven-dimensional M-theory.

\subsection{Type II String Theory on a Calabi-Yau Threefold}

One way to obtain four-dimensional $\mathcal{N}=2$ supergravity from string theory is by compactifying type II string theory on a complex-three-dimensional Calabi-Yau manifold. Such spaces are Kähler manifolds with vanishing first Chern class.] Since they have holonomy group $S U(3)$ they posess Killing spinors and the number of supercharges of the four-dimensional theory is $1 / 4$ of the number of supercharges of the ten-dimensional theory. In the following we review some facts about Calabi-Yau compactifications of string theory. For a more complete account we refer to the reviews 155, 156, 157, 158].

The four-dimensional theory has a massless spectrum that consists of the $\mathcal{N}=2$ supergravity multiplet plus a model-dependent number of abelian vector multiplets and neutral hypermultiplets. A hypermultiplet contains two Weyl spinors and four real scalars as its on-shell degrees of freedom. The kinetic term of the hypermultiplet scalars is a non-linear sigma-model with a target space that is restricted to be quaternionic by local $\mathcal{N}=2$ supersymmetry [159]. Local $\mathcal{N}=2$ supersymmetry forbids neutral couplings between vector and hypermultiplets 16. Since the string compactification for generic moduli only contains gauge-neutral fields, the total moduli space factorizes into a product of the special Kähler manifold of vector multiplet moduli and the quaternionic manifold of hypermultiplet moduli. This factorization breaks down at special points in the moduli space, where the Calabi-Yau manifold becomes singular in such a way that the moduli space metric and the low energy effective action are singular, too. 2 The most popular case of such a singularity is the conifold singularity [160]. The conifold point and other more complicated singular points in the moduli space are at finite distance from regular points. The fact that string backgrounds can become singular under finite changes of the parameters was long considered as a severe problem. Then it was observed by Strominger that the conifold singularity could be physically explained by the presence of a charged hypermultiplet that becomes massless at the conifold point 161]. The additional massless state corresponds to a type IIB threebrane, which is wrapped on the three-cycle which degenerates at the conifold point.

\footnotetext{
${ }^{1}$ We also include the condition $h_{1,0}=0$ in order to exclude from the definition the six-torus $T^{6}$ and $K 3 \times T^{2}$, where $K 3$ denotes the $K 3$ surface.

${ }^{2}$ There exist milder singularities, such as orbifold singularities and flop transitions, which do not give rise to singularities of string theory.
} 
When the additional state is taken into account, all physical quantities behave smooth at the conifold point. Subsequent work generalized this to other types of singularities. In particular it was shown that more complicated singularities correspond to multicritical points in the scalar potential of $\mathcal{N}=2$ supergravity coupled to charged matter [162]. At these points one has the option to go to different branches of the theory, such as the Coulomb and Higgs branch, which we discussed in chapter 2. The corresponding geometric mechanism is that a singularity can be resolved in several non-equivalent ways. The transition between branches of the scalar potential corresponds to a topological phase transition, i.e. a change in the topology of the Calabi-Yau manifold. In such transitions physics, or more precisely the low energy effective action is smooth. We refer to [157] for a review and references. In the following we will only consider generic compactifications where all fields in the Lagrangian are gauge-neutral and the moduli space factorizes into a vector multiplet and a hypermultiplet part.

In type IIA compactifications the numbers $N_{V}, N_{H}$ of vector and hypermultiplets are given by

$$
N_{V}=h_{1,1} \text { and } N_{H}=h_{2,1}+1,
$$

where $h_{1,1}, h_{2,1}$ are Hodge numbers of the Calabi-Yau manifold. Part of the scalar fields are geometric moduli of the internal space. The $h_{1,1}$ vector multiplet moduli describe deformations of the Kähler structure and of the internal part of the stringy $B$-field. Both data can be conveniently combined into the so-called complexified Kähler structure. Among the $4 h_{2,1}$ real hypermultiplet moduli $2 h_{2,1}$ describe deformations of the complex structure whereas the other $2 h_{2,1}$ moduli come from the fields of the Ramond-Ramond sector. There is one additional hypermultiplet which is always present, even when the Calabi-Yau manifold has a unique complex structure, $h_{2,1}=0$. It is called the universal hypermultiplet because of its independence from Calabi-Yau data. One of its scalars is the dilaton $\phi$, whose vacuum expectation value is related to the four-dimensional IIA string coupling by $g_{I I A}=e^{\langle\phi\rangle}$. In addition it contains the stringy axion, which is obtained from the space-time part of the $B$-field by Hodge duality, and two scalars from the Ramond-Ramond sector.

In type IIB compactifications the role of vector and hypermultiplets is reversed,

$$
N_{V}^{(B)}=h_{2,1} \text { and } N_{H}^{(B)}=h_{1,1}+1 .
$$

By mirror symmetry type IIB string theory on a Calabi-Yau manifold is equivalent to type IIA string theory on a so-called mirror manifold [163, 164, see 165, 157 for a review. A Calabi-Yau manifold and its mirror are related by exchanging the roles of the complex structure moduli and (complexified) Kähler moduli spaces.[] In particular the Hodge numbers are related by $\widetilde{h}_{1,1}=h_{2,1}$ and $\widetilde{h}_{2,1}=h_{1,1}$. Due to mirror symmetry we can restrict our attention to one of the two type II theories. For our purposes it is convenient to consider the type IIA theory.

The part of the effective Lagrangian that we are interested in is the vector multiplet sector which is encoded in the function $F(X, \widehat{A})$. By expansion of the function,

$$
F(X, \widehat{A})=\sum_{g=0}^{\infty} F^{(g)}(X) \widehat{A}^{g},
$$

one finds the prepotential $F^{(0)}(X)$ which describes the minimal part of the Lagrangian and the coefficients $F^{(g \geq 1)}(X)$ of higher derivative couplings of the form $C^{2} T^{2 g-2}$. In string perturbation theory one can compute on-shell scattering amplitudes and obtain an effective action. This action has ambiguities because string perturbation theory has only access to on-shell quantities. Therefore terms in the effective action are only known up to terms which vanish on-shell. For example the difference between the square of the Riemann tensor and the square of the

\footnotetext{
${ }^{3}$ The precise statement of mirror symmetry is that IIA and IIB string theory in the corresponding string backgrounds are equivalent. String backgrounds are defined in terms of conformal field theories. It is important to take into accoung stringy $\alpha^{\prime}$ corrections of the classical geometry. Moreover one has to include regions of the moduli space, so-called non-geometric phases, which do not have a geometrical interpretation in terms of a Calabi-Yau sigma model.
} 
Weyl tensor vanishes in a Ricci-flat background and therefore string perturbation cannot decide whether the curvature squared term with coupling $F^{(1)}(X)$ involves the Riemann or the Weyl tensor. We can invoke the off-shell formalism of chapter 3 to identify this term as the square of the Weyl tensor. Conversely, the effective supergravity Lagrangian of chapter 3 needs the function $F^{(1)}(X)$ as input from string theory, because supergravity is not a consistent quantum theory. As discussed in chapter 3 the relation between the auxiliary $T$-field and the graviphoton is complicated in the off-shell formulation. In [166 a relation is found by requiring that the effective action reproduces the on-shell scattering amplitudes. In this context the graviphoton is defined through its vertex operator.

The string computation involves the physical scalars $z^{A}$ rather then the sections $X^{I}$. In order to rewrite the couplings $F^{(g)}(X)$ we introduce the holomorphic sections $X^{I}(z)$ by

$$
X^{I}=m_{\text {Planck }} e^{\frac{1}{2} K(z, \bar{z})} X^{I}(z),
$$

where $K(z, \bar{z})$ is the Kähler potential. Note that we have restored the Planck mass in order to be able to do dimensional analysis later. We go to special coordinates $z^{A}$ by imposing $X^{0}(z)=1, X^{A}(z)=z^{A}$. Next we use that $F^{(g)}(X)$ is homogenous of degree $2-2 g$ and define

$$
\mathcal{F}^{(g)}(z)=i\left[m_{\text {Planck }}\right]^{g-1} e^{-(1-g) K} F^{(g)}(X) .
$$

In the language of chapter 3 the quantity $\mathcal{F}^{(g)}(z)$ is a holomorphic section of $\mathcal{L}^{2(1-g)}$, whereas $F^{(g)}$ is a covariantly holomorphic section of $\mathcal{P}^{2(1-g)}$.

In order to display the dependence of $F^{(g)}(X)$ on the string coupling $g_{S}$, we have to replace the Planck mass by the string mass $m_{\text {String }}=m_{\text {Planck }} g_{S}$. This yields

$$
F^{(g)}(X)=-i m_{\text {String }}^{2-2 g} g_{S}^{-2+2 g} e^{(1-g) K} \mathcal{F}^{(g)}(z) .
$$

Next we use that the string coupling is given by the vacuum expectation value of the dilaton and that in type II compactifications the dilaton sits in a hypermultiplet. The Kähler potential $K$ and the function $\mathcal{F}^{(g)}(z)$ depend only on the Kähler moduli and not on hypermultiplet moduli like the dilaton. Therefore the factorization of the moduli space into vector and hypermultiplet moduli has the far reaching consequence that the dependence of the terms in the vector multiplet part of the Lagrangian is very simple: $F^{(g)}(X)$ depends on the string coupling only through the factor $g_{S}^{2-2 g}$ required by dimensional analysis. This term is precisely generated at the $g$-loop level of type II perturbation theory. Supersymmetry leaves no room for perturbative or nonperturbative corrections. In particular the prepotential $\mathcal{F}^{(0)}(z)$ can be computed exactly at string tree level. But quantities related to the hypermultiplet sector can depend on the dilaton in a non-trivial way and are therefore subject to complicated perturbative and non-perturbative quantum corrections. But this will not concern us here, since black hole solutions exclusively depend on quantities in the vector multiplet sector.

In string theory we have a second type of corrections in addition to loop corrections, namely $\alpha^{\prime}$-corrections. At given loop order these are additional stringy corrections to point particle behaviour, which give rise to higher derivative terms in the effective action. These terms are suppressed by additional powers of the string scale, which can be either parametrized by the string mass $m_{\text {String }}$ or by the parameter $\alpha^{\prime}$ which has dimension length squared, $m_{\text {String }}^{-1} \sim \sqrt{\alpha^{\prime}}$. One way to understand $\alpha^{\prime}$-corrections is to look at strings moving in non-trivial background fields, in particular in a curved background geometry. In this case the worldsheet action of the string is a non-linear sigma-model, and therefore the two-dimensional worldsheet theory has non-trivial quantum corrections. The role of the dimensionless loop counting parameter is played by the curvature of the manifold measured in units of $\alpha^{\prime}$. In the case of strings propagating in a Calabi-Yau manifold the information about the size and curvature of the manifold is encoded in the Kähler moduli. Since the Kähler moduli sit in vector multiplets, all quantities in this sector have a non-trivial dependence on the Kähler structure and are subject to perturbative and non-perturbative $\alpha^{\prime}$-corrections. The perturbative corrections are given by world-sheet loops, whereas the non-perturbative corrections are due to world-sheet instantons. 
World-sheet instantons arise from non-trivial embeddings of the string world-sheet into the Calabi-Yau manifold and produce saddle points in the string path integral. More specifically the string world sheet is a genus $g$ Riemann surface and can be holomorphically mapped onto twocycles inside the Calabi-Yau space. The mappings are classified by their degrees $d_{1}, \ldots, d_{h_{1,1}}$, where the degree $d_{i}$ specifies how many times the world sheet is wrapped around the $i$-th generator of the homology group $H_{2}(X, \mathbb{Z})$. These generators provide a basis in which every two-cycle can be expanded (modulo homology). The number of genus $g$ instantons, i.e. the number of distinct holomorphic mappings of the genus $g$ world-sheet onto holomorphic twocycles with degrees $d_{1}, \ldots, d_{h_{1,1}}$ is denoted by $n_{d_{1}, \ldots d_{h_{1,1}}}^{(g)}$. These numbers 'count' holomorphic genus $g$ curves of given homology and degrees in the Calabi-Yau manifold f

Obviously it is a hopeless task to compute all the world sheet intstanton corrections explicitly term by term. But here mirror symmetry enables one to find the full result. When switching from type IIA theory to type IIB theory on the mirror manifold the vector multiplet moduli are now complex structure moduli. But since the world-sheet corrections are controlled by the Kähler moduli, all quantities in the complex structure moduli space do not receive $\alpha^{\prime}$-corrections and can be calculated exactly at tree level in $\alpha^{\prime}$. In geometric terms this means that while the Kähler structure of the Calabi-Yau manifold is modified when probed by a string instead of a point particle, the complex structure is not. One can now try to compute the functions $\mathcal{F}^{(g)}(z)$ in the type IIB theory and then switch to IIA variables by the mirror map that connects the two theories. This is still a complicated problem, because one has to compute a string $g$ loop diagram in a Calabi-Yau background geometry. In the case of the prepotential $\mathcal{F}^{(0)}(z)$ special geometry can be exploited. The holomorphic section $\left(X^{I}(z), F_{J}(X(z))\right)$ is related to the periods of the holomorphic $(3,0)$-form of the Calabi-Yau manifold and the so-called PicardFuchs equations can be used to compute it 160, 168]. The computation of the function $\mathcal{F}^{(1)}(z)$ 169] is related to the supersymmetric index of [170]. The higher functions $\mathcal{F}^{(g)}(z), g>1$ satisfy a holomorphic anomaly equation and information about them can be obtained by using various techniques in particular the topological twisting of the string sigma model and the computation of special (so-called topological) scattering amplitudes [167, 166].

Let us next have a look at concrete formulae for the functions $\mathcal{F}^{(g)}(z)$. We will use the type IIA description. Since the Calabi-Yau metric has to be positive definite, the space of Kähler deformations has the structure of a cone. We use the convention that the Kähler moduli sit in the imaginary part of the complex field $z^{A}$. The variables are chosen such that the Kähler cone is given by

$$
\operatorname{Im} z^{A}>0 \text {. }
$$

The real parts of the $z^{A}$ contain the zero modes of the internal part of the stringy $B$-field. The gauge symmetry associated with this field translates into a Peccei-Quinn symmetry of $\operatorname{Re} z^{A}$.

We are now prepared to present the general form of the IIA prepotential [160, 168]:

$$
\mathcal{F}_{I I A}^{(0)}=-i \frac{1}{6} C_{A B C} z^{A} z^{B} z^{C}-i \frac{\chi \zeta(3)}{2(2 \pi)^{3}}+i \frac{1}{(2 \pi)^{3}} \sum_{\left\{d_{i}\right\}} n_{\left\{d_{i}\right\}}^{(0)} \operatorname{Li}_{3}\left(\exp \left[i \sum_{A} d_{A} z^{A}\right]\right),
$$

where $\left\{d_{i}\right\}:=\left\{d_{1}, \ldots, d_{h_{1,1}}\right\}$. In this formula $\mathrm{Li}_{3}$ denotes the third polylogarithmic function, see appendix $\mathrm{E}$. The coefficients $C_{A B C}, \chi, n_{\left\{d_{i}\right\}}^{(0)}$ are topological data of the Calabi-Yau space, namely its triple intersection numbers, the Euler number and the rational or genus zero worldsheet instanton numbers, respectively. The first term arises at tree level in $\alpha^{\prime}$, whereas the second term is a loop correction. The third term encodes the world-sheet instanton corrections that we discussed above. The classical or large volume limit corresponds to taking all Kähler moduli to be large, $\operatorname{Im} z^{A} \rightarrow \infty$. This corresponds to a region deep inside the Kähler cone, as opposed to the boundaries $\operatorname{Im} z^{A}=0$. Geometrically, large Kähler moduli mean that the sizes of

\footnotetext{
${ }^{4}$ This is at least the intuitive interpretation of these numbers. The mathematics behind it is more complicated. For example, holomorphic curves are not necessarily isolated but can form continuous families, so that one needs to generalize the notion of counting. We will not need to describe this here and refer the interested reader to the literature, see for example 167 .
} 
the manifold and of all its two- and four cycles are large (in units of $\alpha^{\prime}$ ) and therefore curvature is small. In the limit $\operatorname{Im} z^{A} \rightarrow \infty$ the world-sheet instanton corrections are exponentially small, whereas the classical cubic term is very large. Since the leading classical piece contains the triple intersection form, one can interpret the $\alpha^{\prime}$-corrections as a 'quantum deformation' of the standard triple intersection form. Thus the formula for the prepotential summarizes in which way the geometry seen by strings differs from classical geometry.

Let us next display the structure of the $C^{2}$-coupling [169]:

$$
\begin{aligned}
\mathcal{F}_{I I A}^{(1)(\text { hol })}(z)= & -i \sum_{A} z^{A} c_{2 A}-\frac{1}{\pi} \sum_{\left\{d_{i}\right\}}\left\{12 n_{d_{1}, \ldots}^{(1)} \log \left[\widetilde{\eta}\left(\exp \left[i \sum_{A} d_{A} z^{A}\right]\right)\right]\right. \\
& \left.+n_{\left\{d_{i}\right\}}^{(0)} \log \left[1-\exp \left(i \sum_{A} d_{A} z^{A}\right)\right]\right\}
\end{aligned}
$$

where $\widetilde{\eta}(q)=\prod_{m=1}^{\infty}\left(1-q^{m}\right)$. Among the topological data that enter this time are the second Chern class numbers $c_{2 A}$, which are the expansion coefficients of the second Chern class in a basis of $H^{4}(X, \mathbb{Z})$ that is dual to the chosen basis of $H_{2}(X, \mathbb{Z})$. The corresponding term arises at tree level in $\alpha^{\prime}$ and is dominating in the large volume limit. There are no $\alpha^{\prime}$-loop corrections but world sheet instantons of genus 0 and 1, called rational and elliptic instantons, respectively. Naively one would only expect elliptic instantons according to the discussion given above. The appearence of rational instantons is related to one of the many subtleties that we do not discuss explicitly here, namely to the proper treatment of degenerate curves, where the so-called 'bubbling phenomenon' has to be taken into account [169].

Also note that we put an additional label 'hol' on the function in order to notify that we have only displayed the holomorphic part of $\mathcal{F}^{(1)}(z)$. The function also has a non-holomorphic part which is determined by the so-called holomorphic anomaly equation [169]. From the point of view of the string world sheet the non-holomorphic contribution comes from a contact term and the holomorphic anomaly equation is a modified superconformal Ward identity. From the space-time point of view the fact that the full coupling $\mathcal{F}^{(1)}(z)$ is non-holomorphic is due to non-trivial infrared physics 171. As we saw in chapter 3 the couplings occuring in the most general local supersymmetric Lagrangian are necessarily holomorphic functions of the moduli. In quantum field theory the term effective action refers to the generating functional of 1PI Greens functions. If massless particles are present this effective action is in general non-local, due to the fact that one integrates out all modes, including the massless ones. This has the consequence that the physical couplings of supersymmetric field theories depend in a non-holomorphic way on the moduli, as discussed for gauge couplings in [172, 173] and for gravitational couplings in [171, 166]. In string perturbation theory one computes on-shell amplitudes, which automatically incorporate the loops of massless particles. Therefore the couplings extracted from such a computation are the physical, non-holomorphic couplings. A local supersymmetric Lagrangian cannot properly account for the non-holomorphic part of the coupling. The corresponding effective action is therefore interpreted as a Wilsonian effective action, i.e. an effective action where only the massive modes above a certain infrared cut-off have been integrated out. The corresponding holomorphic couplings are called Wilsonian couplings. Note that these remarks do not only apply to $\mathcal{N}=2$ compactifications but to supersymmetric string effective actions in general, see [174] for an overview.

Finally we have to discuss the higher couplings $\mathcal{F}^{(g>1)}(z)$ 167, 166, 175, 176]. These functions do not have a contribution at tree level in $\alpha^{\prime}$ and the leading term is an $\alpha^{\prime}$-loop correction which yields a term proportional to $\chi \zeta(3)$. The non-perturbative terms involve genus $g$ world sheet instantons, but there are additional subtleties such as 'bubblings' which lead to further contributions. Like the function $\mathcal{F}^{(1)}(z)$ all the higher functions receive non-holomorphic con-

\footnotetext{
${ }^{5}$ Though the name quantum geometry is common for this deformation, one should keep in mind that 'quantum' refers to world-sheet and not to space-time properties. One might prefer to call this 'stringy geometry' as opposed to 'point particle geometry', i.e. the geometry seen by point particles.
} 
tributions, which are subject to a hierarchy of holomorphic anomaly equations. In chapter 6 we will discuss the contribution of non-holomorphic terms to the black hole entropy.

The derivation of both the macroscopic and microscopic black hole entropy that we will discuss in the next chapter is performed in the large volume limit. The most basic approximation consists of taking only the terms which arise at tree level in $\alpha^{\prime}$. In this case only the leading parts of $\mathcal{F}^{(0)}(z)$ and $\mathcal{F}^{(1)}(z)$ contribute,

$$
\mathcal{F}^{(0)}(z)=-i \frac{1}{6} C_{A B C} z^{A} z^{B} z^{C} \text { and } \mathcal{F}^{(1)}(z)=-i c_{2 A} z^{A} .
$$

\subsection{M-Theory on a Calabi-Yau Threefold times $S^{1}$}

The most striking development in the discovery of string dualities was that the strong coupling limit of ten-dimensional IIA string theory is an eleven-dimensional theory, which at low energies is effectively described by eleven-dimensional supergravity [81. The full theory behind this effective theory cannot be a perturbative supersymmetric string theory, because one is beyond the critical dimension $D=10$. The new theory has been called eleven-dimensional M-theory, and subsequent work has led to the conclusion that all five consistent perturbative string theories describe asymptotic expansions around special points in the full moduli space of M-theory [7].

In the duality between ten-dimensional IIA string theory and eleven-dimensional M-theory the ten-dimensional IIA string coupling is related to the (geodesic) radius of the eleventh dimension by

$$
g_{I I A}^{2}=\left(\frac{R_{11}}{L_{11}}\right)^{3}
$$

where $L_{11}$ is the eleven-dimensional Planck length, which is related to the Regge parameter $\alpha^{\prime}$ of IIA string theory by

$$
L_{11}^{3}=\alpha^{\prime} R_{11}
$$

and to the eleven-dimensional gravitational coupling by $\kappa_{11}^{2}=L_{11}^{9}$.

As a consequence of equation (5.11) weak IIA coupling corresponds to a small eleventh dimension, which is invisible in IIA perturbation theory. Conversely large coupling corresponds to the decompactification of the extra dimension and at sufficiently small energies eleven-dimensional supergravity describes the strong coupling behaviour of the IIA string.

Upon compactification on a manifold $X$, IIA string theory on $X$ and M-theory on $X \times S^{1}$ are 'on the same moduli'. This means that they are part of one single theory and describe two different regimes, namely weak ten-dimensional IIA coupling and strong ten-dimensional IIA coupling, respectively.

We consider now the case that $X$ is a Calabi-Yau threefold. The compactification of elevendimensional supergravity on a Calabi-Yau threefold 177 yields minimal five-dimensional supergravity coupled to $N_{V}^{(5)}$ abelian vector and $N_{H}^{(5)}$ neutral hypermultiplets, where

$$
N_{V}^{(5)}=h_{1,1}-1, \quad N_{H}^{(5)}=h_{2,1}+1
$$

and $h_{1,1}, h_{2,1}$ are Hodge numbers of the Calabi-Yau space. By further compactification on a circle one gets four-dimensional $\mathcal{N}=2$ supergravity coupled to $N_{V}=h_{1,1}$ vector and $N_{H}=$ $h_{2,1}+1$ hypermultiplets, which is indeed the same spectrum that one gets by compactification of type IIA string theory on the same threefold.

The vector multiplet couplings are fully specified by the triple intersection numbers $C_{I J K}$ 178, 179. The geometric structure behind the vector multiplet couplings of five-dimensional supergravity is very special geometry 141. In this language the particular feature compared to four-dimensional $\mathcal{N}=2$ supergravity is that the prepotential is purely cubic. This has a nice interpretation in terms of the M-theory limit of the IIA string [180]: In this case the limit of strong ten-dimensional IIA coupling can be reinterpreted as the large volume limit of the Calabi-Yau space. Here 'large' refers to measuring the metric in stringy $\alpha$-units and 
therefore is equivalent to taking the limit $\alpha^{\prime} \rightarrow 0$ in which all perturbative and non-perturbative $\alpha^{\prime}$-corrections vanish. Thus one is left with the classical part of the prepotential.

When studying the theory at finite $S^{1}$ radius, then $\alpha^{\prime}$-corrections are present and correspond in the M-theory language to perturbative and non-perturbative corrections involving the Kaluza-Klein modes and solitons of M-theory. This has recently been used to obtain information about the four-dimensional vector multiplet couplings $\mathcal{F}^{(g)}(z)$ from the M-theory perspective [175, 176]. In particular one-loop calculations involving Kaluza-Klein modes of the five-dimensional theory and wrapped M2-branes have been used to compute the leading parts of $\mathcal{F}^{(g)}(z)$ for all $g$. These computations confirm and extend known results and are sensitive to effects like the 'bubblings' we mentioned in the last section.

Later on we will consider a contribution to the function $F(X, \widehat{A})$ which takes the form $G\left(X^{0}, \widehat{A}\right)$ and encodes subleading contributions from all genera $g$ in the large volume limit. For this function an integral representation was derived in 175 by a computation which is similar to the Schwinger calculation of charged particle creation in an external field. We will discuss how such a contribution modifies the black hole entropy.

\subsection{Heterotic String Theory on $K 3 \times T^{2}$}

So far we constructed four-dimensional $\mathcal{N}=2$ string vacua using theories with 32 supercharges. Alternatively one can start with the heterotic or with the type I string, which only posess 16 supercharges. Then one needs to compactify on a manifold which preserves $1 / 2$ of the supersymmetries of the ten-dimensional theory. The most simple choice, besides singular spaces such as orbifolds, is $K 3 \times T^{2}$, where $K 3$ is a $K 3$-surface and $T^{2}$ is the two-dimensional torus. A $K 3$-surface is a complex Kähler surface with vanishing first Chern class or, in other words, a Calabi-Yau twofold. The holonomy group is $S U(2)$. In contrast to Calabi-Yau threefolds the topology of such surfaces are unique, but deformations of the complex structure and of the (complexified) Kähler structure exist. We refer to [181, 158] for a review of string theory in $K 3$ backgrounds.

Heterotic and type I string theories have a gauge group, $E_{8} \times E_{8}$ or $S O(32)$, in ten dimensions. In order to obtain consistent compactifications one has to switch on a non-trivial gauge field configuration if the space-time geometry is curved. In our case one has to choose an instanton configuration inside the $K 3$. The low-energy effective theory (at generic points in the moduli space) only depends on the topological class of the gauge field, i.e. on the instanton number (the second Chern class of the gauge bundle).

All three options, the heterotic string theories with gauge groups $E_{8} \times E_{8}$ or $S O(32)$ and the type I string theory with gauge group $S O(32)$ are related by dualities. Whereas the two heterotic theories are related by T-duality after compactification on a circle, the type I theory is $S$-dual to the heterotic $S O(32)$ theory. When compactifyed on $K 3 \times T^{2}$ all three theories are 'on the same moduli'. We will choose the perspective of the heterotic $E_{8} \times E_{8}$ theory. Moreover we will restrict ourselves to so-called perturbative vacua, i.e. to compactifications without additional $p$-branes in the compact part of space-time.

By compactification of the $E_{8} \times E_{8}$ theory on $K 3$ one obtains minimal, chiral six-dimensional $\mathcal{N}=(0,1)$ supergravity coupled to $N_{T}^{(6)}=1$ tensor, $N_{V}^{(6)}$ vector and $N_{H}^{(6)}$ hypermultiplets. The resulting models have been studied in the context of F-theory 182, 183, 184.

The tensor multiplet contains the six-dimensional dilaton. In order to obtain a consistent, in particular anomaly-free theory one has to switch on $E_{8} \times E_{8}$ instantons with instanton numbers $N_{I}^{(1)}+N_{I}^{(2)}=24$ in the $K 3$. The gauge group depends on the instanton numbers $\left(N_{I}^{(1)}, N_{I}^{(2)}\right)$ and on the position in moduli space. In six dimensions a vector multiplet does not contain scalars, so that the moduli sit in tensor and hypermultiplets. For the perturbative vacua that we consider here the dilaton is the only tensor multiplet modulus. The hypermultiplet moduli space contains the moduli of the $K 3$ and of the instantons. At a generic positon in moduli space the gauge group and massless spectrum are minimal. We assume to be at a generic point in the hypermultiplet moduli space. Then, the three models with instanton numbers $(12,12),(13,11)$ 
and $(14,10)$ have the same massless spectrum: The gauge group is broken completely, $N_{V}^{(6)}=0$ and there are $N_{H}^{(6)}=244$ neutral hypermultiplets. If the instanton numbers are distributed more asymmetrically, then the gauge group is not broken completely, so that one has $N_{V}^{(6)} \neq 0$.

By compactification on $K 3 \times S^{1}$ one obtains minimal five-dimensional supergravity coupled to $N_{V}^{(5)}=N_{V}^{(6)}+2$ vector multiplets and $N_{H}^{(5)}=N_{H}^{(6)}$ hypermultiplets. One of the additional vector multiplets is related to the usual Kaluza-Klein vector whereas the other is obtained by Hodge dualization of the six-dimensional tensor multiplet. A five-dimensional vector multiplet contains one real scalar. At generic points in the moduli space the gauge group is abelian and the hypermultiplets are neutral. As in the case of five-dimensional M-theory compactifications the vector multiplet couplings are described by very special geometry.

By compactification on $K 3 \times T^{2}$ one obtains four-dimensional $\mathcal{N}=2$ supergravity coupled to $N_{V}^{(4)}=N_{V}^{(6)}+3$ vector and $N_{H}^{(4)}=N_{H}^{(5)}$ hypermultiplets. Again we have gained one vector multiplet by Kaluza-Klein reduction. The scalars in the vector multiplets are now complex.

This counting applies to the three models mentioned above, where the six-dimensional gauge group is completely broken for generic moduli. For the other models with instanton numbers $(15,9),(16,8), \ldots$ one has an unbroken non-abelian group $S U(3), S O(8), \ldots$ in six dimensions. After compactification one can use the two versions of the Higgs mechanim available in fourdimensional $\mathcal{N}=2$ theories ('going to the Coulomb- or Higgsbranch') that we briefly mentioned in chapter 2 to break the gauge group. For generic moduli one is left with abelian vector and neutral hypermultiplets. Thus the effective action is of the type we have studied.

In the case of the models with instanton numbers $(12,12),(13,11)$ and $(14,10)$ we obtain precisely three vector multiplets, and therefore gauge group $U(1)^{4}$, where the extra $U(1)$ is due to the graviphoton. These models are called the three parameter models, because they have three complex vector multiplet moduli.6 The number of neutral hypermultiplets is 244 . Fortunately this sector will not concern us here. The other models have larger abelian gauge groups, which are relics of the six-dimensional gauge group. All models form branches of one single moduli space.

By a slightly modified scheme of compactification one can construct another class of models where an even smaller vector multiplet sector is possible [185]: One first compactifies on $T^{2}$ to $D=8$ and freezes one modulus by imposing that the Kähler modulus $T$ and the complex structure modulus $U$ are equal, $T=U$, so that the model has an enhanced gauge group $S U(2)$ related to the $T^{2}$ in $D=8$. Now one compactifies on $K 3$ and puts instantons both in the $E_{8} \times E_{8}$ and in the $S U(2)$. When distributing the instanton numbers sufficiently symmetric and going to generic moduli one arrives at the so-called two-paramter model with generic gauge group $U(1)^{3}$.

In contradistinction to type II compactifications the dilaton sits in one of the vector multiplets. Therefore the prepotential and all the higher couplings get perturbative and nonperturbative corrections. We will proceed step by step and start with the tree level prepotential.

\subsubsection{The Tree Level Prepotential}

The tree level prepotential takes a universal form that only depends on the number of vector moduli 186]. In order to describe the models in terms of the physical scalars we introduce

$$
\mathcal{F}^{(0)}(z)=i m_{\text {Planck }}^{-1} e^{-K} F^{(0)}(X(z)),
$$

where $K(z, \bar{z})$ is the Kähler potential and $X^{I}(z)$ are the holomorphic sections. In the following we set $m_{\text {Planck }}=1$ and drop the label (0) on the prepotential. We introduce special coordinates $X^{0}(z)=1$ and $X^{A}(z)=z^{A}$. The heterotic tree level potential is purely cubic, $\mathcal{F}(z)=$ $i D_{A B C} z^{A} z^{B} z^{C}$, with a special form of $D_{A B C}$ that we will discuss in a minute. First we remark

\footnotetext{
${ }^{6}$ Since all models are equivalent at the classical level, one sometimes refers to them simply as 'the' three parameter model. It turns out that at the non-perturbative level there are actually two inequivalent models, see below and [184].

${ }^{7}$ We are using the standard parametrization of the moduli of a torus. See for example 33.
} 
that the standard convention in heterotic models is to parametrize the moduli by $S^{A}=-i z^{A}$. Thus it is the imaginary part rather than the real part that has a Peccei-Quinn symmetry. The prepotential takes the form $\mathcal{F}=D_{A B C} S^{A} S^{B} S^{C}$. In order to understand the special form that $D_{A B C}$ takes in heterotic tree level models, let us look at the physical and geometrical interpretation of the three moduli of the three parameter model.

One of the moduli contains the four-dimensional dilaton $\phi^{(4)}$ and the universal axion $a$, which is obtained by dualizing the universal $B_{\mu \nu}$-field. At the supermultiplet level the dilaton originally resides in the tensor multiplet, which, below six dimensions, can be dualized into a vector multiplet. One combines the dilaton and axion into a four-dimensional complex dilaton

$$
S^{1}=S=4 \pi e^{-2 \phi^{(4)}}+i a=\frac{4 \pi}{g_{S}^{2}}-i \frac{\theta}{2 \pi}
$$

where $g_{S}$ is the four-dimensional heterotic tree level coupling and $\theta$ is the $\theta$-angle associated with the universal axion $a$. The other two universal moduli are related to the two-torus. They are the complexified Kähler modulus $S^{2}=T$ and the complex structure modulus $S^{3}=U$ of the torus. The standard notation $S, T, U$ motivates the synonym $S T U$ model for the three parameter model. Models with more vector multiplets have additional moduli $S^{3+i}=V^{i}$, which parametrize Wilson lines on the torus.

We now come back to the prepotential. The tree level part must be linear in $S$ in order to have the right dependence on the string coupling. The quadratic polynomial depending on the geometric moduli $T^{a}=T, U, V^{i}$ is unique:

$$
\mathcal{F}\left(S, T^{a}\right)=-S T^{a} \eta_{a b} T^{b}=-S\left(T U-\sum_{i} V^{i} V^{i}\right) .
$$

Note that

$$
\left(\eta_{a b}\right)=\left(\begin{array}{ccc}
0 & \frac{1}{2} & 0 \\
\frac{1}{2} & 0 & 0 \\
0 & 0 & -\delta_{i j}
\end{array}\right) .
$$

One can now compute the Kähler potential, with the result that the moduli space locally takes the form

$$
\left.\mathcal{M} \sim \frac{S U(1,1)}{U(1)}\right|_{S} \times\left.\frac{S O\left(2, N_{V}-1\right)}{S O(2) \times S O\left(N_{V}-1\right)}\right|_{T, U, V_{i}} .
$$

The first factor is related to the dilaton, which at tree level does not mix with the moduli. For the $S T U$-model one gets

$$
\left.\mathcal{M} \sim \frac{S U(1,1)}{U(1)}\right|_{S, T, U} ^{3}
$$

by using the local isomorphism $S O(2,2) \sim S U(1,1) \times S U(1,1)$.

The global structure of the moduli space is determined by T-duality. We refer to 187 for a review. In compactifications on $S^{1} \mathrm{~T}$-duality relates the radius to its inverse in string units. For torus compactifications this generalizes to a more complicated discrete group, which in particular contains large diffeomorphisms of the tours, the inversion of radii and discrete axion-like shift symmetries of the imaginary parts of the moduli. In heterotic compactifications the group also contains transformations acting on the Wilson lines. In our case the discrete group is $S O\left(2, N_{V}-1, \mathbb{Z}\right)$. In $\mathcal{N}=2$ compactifications T-dualities are realized as a subset of the symplectic transformations [120, 188]. In contradistinction to generic symplectic transformations, T-dualities are true symmetries of the theory, at least at the tree and perturbative level. Therefore physical observables have to be T-duality invariant. In order to display the

\footnotetext{
${ }^{8}$ In the most simple cases the $z^{A}$ parametrize the upper complex half plane, whereas the $S^{A}$ paramatrize the right half plane, $\operatorname{Re} S^{A}>0$.
} 
symplectic action of T-duality, we have to describe the theory in terms of symplectic sections. For simplicity we will consider the $S T U$ model. In this case the prepotential is

$$
F(X(z))=-\frac{X^{1}(z) X^{2}(z) X^{3}(z)}{X^{0}(z)} \leftrightarrow \mathcal{F}(S, T, U)=-S T U
$$

and therefore the holomorphic section, written in special coordinates is

$$
\left(X^{I}, F_{J}\right)=(1, i S, i T, i U,-i S T U, T U, S U, S T) .
$$

It turns out that this parametrization is not the natural one from the point of view of the heterotic string. When computing the associated gauge couplings in the perturbative limit $S \rightarrow \infty$, the coupling of the gauge field in the dilaton vector multiplet blows up, whereas all other gauge couplings go to zero. The natural behaviour, where all gauge couplings are small for $S \rightarrow \infty$, is obtained when going to another section by a symplectic reparametrization,

$$
\left(P^{I}, Q_{J}\right)=(1,-T U, i T, i U,-i S T U, i S, S U, S T) .
$$

Note that $Q_{J}$ is not the gradient of a prepotential $F(P)$. This is the standard example for a section without prepotential [120]. Since the two parametrizations are related to one another in a simple way we find it convenient to refer to (5.20) as 'the prepotential'. The above symplectic transformation amounts to an electric - magnetic duality in the $U(1)$ associated with $X^{1}, F_{1}$. The new symplectic vector of electric and magnetic charges is denoted by

$$
\left(M^{I}, N_{J}\right)=\left(q_{0},-p^{1}, q_{2}, q_{3}, p^{0}, q_{1}, p^{2}, p^{3}\right),
$$

where $q^{I}, p_{J}$ are the electric and magnetic charges of the old section.

We now review how T-duality is realized in the $S T U$ model. The T-duality group is

$$
O(2,2, \mathbb{Z})=\left(S L(2, \mathbb{Z})_{T} \otimes S L(2, \mathbb{Z})_{U}\right) \times \mathbb{Z}_{2}^{T-U} .
$$

The two $S L(2, \mathbb{Z})$ groups act by fractional linear transformations

$$
\begin{array}{ll}
T \rightarrow \frac{a T-i b}{i c T+d}, & U \rightarrow U, \\
T \rightarrow T, & U \rightarrow \frac{a^{\prime} U-i b^{\prime}}{i c^{\prime} U+d^{\prime}} .
\end{array}
$$

where

$$
\left(\begin{array}{ll}
a & b \\
c & d
\end{array}\right), \quad\left(\begin{array}{ll}
a^{\prime} & b^{\prime} \\
c^{\prime} & d^{\prime}
\end{array}\right) \in S L(2, \mathbb{Z}) .
$$

The $\mathbb{Z}_{2}$ group exchanges the Kähler and complex structure modulus

$$
T \leftrightarrow U .
$$

This is mirror symmetry for $T^{2}$. All these transformations leave the dilaton invariant, $S \rightarrow S$.

The above transformations act as symplectic transformations on the sections. In the heterotic basis $\left(P^{I}, Q_{J}\right)$ all T-duality transformations take the particular form

$$
\Gamma_{\text {class }}=\left(\begin{array}{cc}
\mathbf{U} & \mathbb{O} \\
\mathbb{O} & \mathbf{U}^{T,-1}
\end{array}\right),
$$

where $\mathbf{U} \in O(2,2, \mathbb{Z})$. Transformations of this special structure leave the Lagrangian invariant.? This is related to the fact that the prepotential does not have non-trivial monodromies at the classical level.

\footnotetext{
${ }^{9}$ This does not only hold for T-duality transformations but for all symplectic transformations with $\mathbf{U} \in$ $G L(4, \mathbb{Z})$.
} 
The prepotential and section of the classical $S T U$-model obviously have a higher symmetry. The corresponding triality group

$$
\left(S L(2, \mathbb{Z})_{T} \otimes S L(2, \mathbb{Z})_{U} \otimes S L(2, \mathbb{Z})_{S}\right) \times \mathbb{Z}_{2}^{T-U} \times \mathbb{Z}_{2}^{S-T} \times \mathbb{Z}_{2}^{S-U}
$$

contains in addition to the full T-duality group also S-duality

$$
S \rightarrow \frac{a S-i b}{i c S+d}, T \rightarrow T, U \rightarrow U
$$

and the exchange symmetries

$$
S \leftrightarrow T, \quad S \leftrightarrow U .
$$

S-duality acts on the heterotic section by

$$
\Gamma=\left(\begin{array}{cc}
a \mathbb{I} & b \mathbb{H} \\
c \mathbb{H} & d \mathbb{I}
\end{array}\right),
$$

where $\mathbb{H}=\eta \oplus \eta$ and

$$
\eta=\left(\begin{array}{ll}
0 & 1 \\
1 & 0
\end{array}\right) .
$$

Such symplectic transformations are not invariances of the Lagrangian. In particular they map small to large string coupling. The symplectic matrices for the two exchange symmetries involving $S$ are of the same type. Note that it is not guaranteed or even likely that such transformations are symmetries of the full non-perturbative theory. For specific models it is known that discrete non-perturbative $S-T$ exchange symmetries exist [189, 190, 191. But such symmetries have to be established case by case using heterotic - type II duality (to be reviewed below). In generic $\mathcal{N}=2$ models one does not expect S-duality at the non-perturbative level. Nevertheless there are several situations where the tree-level formulae displayed above are useful. First we can describe a subsector of a heterotic $\mathcal{N}=4$ compactification. In this case one has evidence for unbroken S-duality at the non-perturbative level [30]. Moreover the full triality group of the $S T U$-model is related to heterotic - IIA - IIB triality present in a subsector of four-dimensional $\mathcal{N}=4$ compactifications 192]. Second there are so called finite or $\mathcal{N}=4$ like $\mathcal{N}=2$ models, like the FHSV model [193], which exhibit S-duality. Finally the triality group simply is a symmetry of the tree level $S T U$-model and therefore physical observables like the black hole entropy should be invariant when computed in the classical approximation. We will see that this is indeed the case.

At special points in the vector multiplet moduli space one obtains non-abelian gauge groups. In the $S T U$-model a $U(1)^{2}$ part of the abelian gauge group is enhanced to $S U(2) \times U(1)$ on the subspace $T=U$ whereas it is enhanced to $S U(2)^{2}$ and $S U(3)$ at $T=U=1$ and $T=U=e^{i \pi / 6}$, respectively. Similarly one obtains larger non-abelian gauge groups in the other models by switching off the Wilson lines. The points of enhanced, non-abelian gauge symmetry are fixed points under some of the T-duality transformations. The fixed point transformations can be identified with the Weyl group of the non-abelian group. This indicates that T-duality is a discrete remnant of a local gauge symmetry of string theory.

\subsubsection{The Perturbative Heterotic Prepotential}

Let us next review the perturbative corrections to the prepotential 120, 188, 194, 195. The one loop contribution $h\left(T^{a}\right)$ to the prepotential is independent of $g_{S}$ and therefore it can only depend on the (geometric) moduli $T^{a}$ but not on the dilaton $S$ :

$$
\mathcal{F}_{1 \text { loop }}\left(S, T^{a}\right)=-S T^{a} T^{b} \eta_{a b}+h\left(T^{a}\right) .
$$


The function $h\left(T^{a}\right)$ can be computed in string perturbation theory and takes the following form 195:

$$
h\left(T^{a}\right)=p\left(T^{a}\right)-c-d \sum_{n_{a} \in \Gamma} c\left(n_{a}\right) \operatorname{Li}_{3}\left(e^{-n_{a} T^{a}}\right) .
$$

The first term $p\left(T^{a}\right)$ is a cubic polynomial in $T^{a}$ which arises at $\alpha^{\prime}$-tree level. In the case of the $S T U$-model the combined tree and one loop cubic part of the prepotential is

$$
\mathcal{F}(S, T, U)=-S T U-\frac{1}{3} U^{3} .
$$

To be precise this is the expression in the region of moduli space where $\operatorname{Re} T>\operatorname{Re} U$. This specification is necessary since it turns out that the full one-loop function has branch cuts. In the other 'Weyl chamber', where $\operatorname{Re} U>\operatorname{Re} T$, the cubic part is $\mathcal{F}(S, T, U)=-S T U-\frac{1}{3} T^{3}$.

The second term $c$ is an $\alpha^{\prime}$-loop correction. It is a constant and in the $S T U$-model it is proportional to $c(0) \zeta(3)$, where $c(0)$ is defined below in (5.38). The third term represents nonperturbative contributions in $\alpha^{\prime}$. At this point we have to explain the origin of the $\alpha^{\prime}$-corrections. Above we argued that $\alpha^{\prime}$-corrections are related to Kähler moduli and we also emphasized that the vector and hypermultiplet moduli spaces factorize. Non-trivial $\alpha^{\prime}$ corrections are to be expected from the Kähler moduli of the $K 3$, because this space has non-trivial curvature. But these moduli sit in hypermultiplets, so how can they contribute to the vector multiplet sector? The reason is as follows [195]: The loop corrections to the prepotential depend on BPS states, only. But in $\mathcal{N}=2$ theories the number of BPS states is not conserved in moduli space. As we explained in chapter 2 short vector multiplets and hypermultiplets, which both are BPS multiplets, can combine into long vector multiplets, which are not BPS mulitplets. When moving through moduli space the number of BPS states changes in a very complicated, 'chaotic' manner. But the difference of the number of short vector and hypermultiplets is conserved and it turns out that $h\left(T^{a}\right)$ only depends on this quantity. Moreover the correction does not explicitly depend on the $K 3$-moduli and can be computed in an orbifold limit of the $K 3$. Let us now investigate the third term of the prepotential more closely: $d$ is an overall constant. The sum runs over a model dependent set of integers denoted by $\Gamma$ and the coefficients $c\left(n_{a}\right)$ encode the corrections. The structure behind these coefficients is an algebraic one: One can associate an infinite dimensional Lie algebra, called the BPS algebra with the BPS states. In particular cases this algebra is a generalized Kac Moody algebra in the sense of Borcherds 195. The set $\Gamma$ parametrizes the positive roots of the BPS algebra. As we already remarked above the explicit expressions for the prepotential refer to subregions of the moduli space such as $\operatorname{Re} T>\operatorname{Re} U$. These regions define the Weyl chambers of the BPS algebra. Furthermore, there is a deep relation between the BPS algebra and automorphic forms of the T-duality group. Since Tduality is a symmetry of string theory to all orders in perturbation theory, the perturbative prepotential must transform in an appropriate way. This is encoded in the above formula by the fact that the coefficients $c\left(n_{a}\right)$, where $n_{a}$ runs over the positive roots of the BPS algebra are expansion coefficients of automorphic forms. To give an explicit example we consider the $S T U$-model and display the non-cubic part (thus including the constant part) 195:

$$
\mathcal{F}=\cdots-\frac{1}{(2 \pi)^{4}}\left(\sum_{k, l \geq 0} c(k l) \operatorname{Li}_{3}\left(e^{-2 \pi(k T+l U)}\right)+\operatorname{Li}_{3}\left(e^{-2 \pi(T-U)}\right)\right) .
$$

In this case the coefficients $c(n)$ are defined by

$$
\frac{E_{4} E_{6}}{\eta^{24}}=\sum_{n \geq-1} c(n) q^{n}=\frac{1}{q}-240-141444 q-8529280 q^{2}-\cdots,
$$

where $E_{4}, E_{6}$ are normalized Eisenstein series and $\eta$ is the Dedekind $\eta$-function, see appendix D. In the above expression for the prepotential we separated the part that describes the behaviour at $T=U$, which is a branch locus of the function. The branch cut is related to the presence of two extra charged massless vector multiplets which enhance one of the $U(1)$ gauge groups 
to $S U(2)$. The gauge couplings calculated from the prepotential contain the corresponding threshold correction. Near the branch cut the threshold correction diverges logarithmically like $\log (T-U)$, because $T-U$ is the associated Higgs field. This form is correct up to finite terms, which are needed to make the threshold correction compatible with T-duality. The covariant form of the threshold correction is $\log (j(i T)-j(i U))$, where $j(z)$ is the modular invariant $j$-function. For generic $T \simeq U$ the expression $j(i T)-j(i U)$ behaves like $T-U$. There are, however, the special points $T=U=1$ and $T=U=e^{i \pi / 6}$ in the moduli space, where one finds the higher rank non-abelian gauge groups $S U(2)^{2}$ and $S U(3)$. Instead of two extra vector multiplets one gets four and six extra multiplets, respectively. The behaviour of the $j$ function and of its derivatives at $z=1, e^{i \pi / 6}$ precisely captures this. Namely $j(i T)-j(i U) \simeq(T-U)^{2}$ for $T \simeq U \simeq 1$, whereas $j(i T)-j(i U) \simeq(T-U)^{3}$ for $T \simeq U \simeq e^{i \pi / 6}$. Therefore the coefficients of the threshold corrections $\log (j(i T)-j(i U))$ have relative weights $1: 2: 3$, reflecting the numbers of extra states at the threshold [33].

In the above example we saw how a particular T-duality transformation acts on the prepotential as a monodromy transformation. More generally the whole T-duality group now acts as a group of non-trivial monodromies. It is convenient to consider the action of the monodromy transformations on the symplectic section instead of looking at the action on the prepotential. In the heterotic basis for the section this takes the form

$$
\Gamma_{\text {pert }}=\left(\begin{array}{cc}
\mathbf{U} & \mathbb{O} \\
\mathbf{U}^{T,-1} \Lambda & \mathbf{U}^{T,-1}
\end{array}\right),
$$

where $\mathbf{U}$ is the $O\left(2, N_{V}-1, \mathbb{Z}\right)$ matrix of classical T-duality wheras the symmetric matrix $\Lambda$ encodes the perturbative modification. Symplectic transformations of the type (5.39) are called 'perturbative transformations'. They have the special property that the Lagrangian is invariant up to a total derivative.19 This shows explicitly that T-duality transformations are true symmetries at the perturbative level.

Perturbative symplectic transformations such as T-dualities have the property that the upper components $P^{I}$ of the symplectic section still transform like under the corresponding classical transformation, whereas the lower components $Q_{J}$ have a modified transformation law. This implies that the geometric moduli $T^{a}=T, U, V^{i}$ transform according to the classical T-duality rules, whereas the conjugated variables $F_{I}, I \neq 1$ transform in a modified way. This precisely reflects that the prepotential now has non-trivial monodromy properties. The group structure of the perturbative monodromy group was analysed in [194], with the result that the reflections with respect to the special loci are promoted to braidings. This precisely accounts for the non-trivial monodromies around the branch loci.

The dilaton $S$, which was invariant under classical T-duality sits in a lower component of the heterotic section. Therefore its transformation behaviour is modified, and $S$ transforms non-trivially under T-duality at the quantum level. Moreover the metric of the moduli space does not factorize any more into a direct product. The Kähler potential now takes the form

$$
K=-\log \left[(S+\bar{S})+V_{G S}\left(T^{i}, \bar{T}^{i}\right)\right]-\log \left[\left(T^{i}+\bar{T}^{i}\right)\left(T^{j}+\bar{T}^{j}\right) \eta_{i j}\right],
$$

where $V_{G S}\left(T^{i}, \bar{T}^{i}\right)$ is the so-called Green-Schwarz term

$$
V_{G S}\left(T^{i}, \bar{T}^{i}\right)=\frac{2(h+\bar{h})-\left(T^{k}+\bar{T}^{k}\right)\left(\partial_{T^{k}} h+\partial_{\bar{T}^{k}} \bar{h}\right)}{\left(T^{i}+\bar{T}^{i}\right)\left(T^{j}+\bar{T}^{j}\right) \eta_{i j}},
$$

which encodes the dilaton - moduli mixing 188. At the tree level the dilaton was connected to the string coupling in a simple way. At the loop level this relation has to be modified, because the string coupling has to be T-duality invariant, whereas the dilaton is not. The correct, T-duality invariant, perturbative string coupling can be defined, by using the fact that the

\footnotetext{
10 This is still true when $\mathbf{U}$ is not a T-duality transformation, but a general matrix $\mathbf{U} \in G L\left(N_{V}+1, \mathbb{Z}\right)$.
} 
Green-Schwarz term precisely compensates the non-invariance of $S$ :

$$
\frac{4 \pi}{g_{\text {pert }}^{2}}=\frac{1}{2}\left(S+\bar{S}+V_{G S}\left(T^{i}, \bar{T}^{i}\right)\right) .
$$

Since the new perturbative coupling is related to the special coordinates $T^{i}$ in a non-linear way, the simple relation to the $\mathcal{N}=2$ supermultiplet structure is lost. This is the price to be payed for having an invariant coupling. [1]

In the above paragraphs we already used the expression 'perturbative' synonymous to 'oneloop'. This is correct because there are no higher loop corrections to the prepotential:

$$
\mathcal{F}_{\text {pert }}\left(S, T^{a}\right)=\mathcal{F}_{1 \text { loop }}\left(S, T^{a}\right) .
$$

The underlying non-renormalization theorem is easily understood in terms of string theory and of the holomorphicity properties of the $\mathcal{N}=2$ action 188. The string coupling is inversely related to the real part of the dilaton, up to a moduli dependent correction, the Green-Schwarz term. The $g$-loop contribution to the Lagrangian is proportional to $g_{S}^{-2+2 g} \sim(\operatorname{Re} S)^{1-g}$. In string theory the dilaton $S$ has a continuous Peccei-Quinn symmetry, which is not broken in perturbation theory. But $\mathcal{N}=2$ supersymmetry implies that the action can be derived from a prepotential, which depends holomorphically on special coordinates such as $S$. Higher loop corrections to the prepotential would either violate the Peccei-Quinn symmetry, through negative powers of $S$ in the prepotential, or violate holomorphicity, by a prepotential that depends on Re $S$ rather than on $S$. Therefore there cannot be higher loop contributions to the prepotential, but only tree and one loop contributions, which are compatible with both Peccei-Quinn symmetry and holomorphicity.

\subsubsection{The Non-Perturbative Heterotic Prepotential}

At the non-perturbative level the prepotential receives further corrections by space-time instantons. Such contributions depend on the dilaton in the form $e^{-S}$ :

$$
\mathcal{F}\left(S, T^{a}\right)=\mathcal{F}_{\text {pert }}\left(S, T^{a}\right)+f^{N P}\left(e^{-S}, T^{a}\right) .
$$

In string theory space-time instantons result from Euclidean $p$-branes, which are wrapped on $p+1$ cycles of the compactification manifold. In the heterotic string theory the instantons result from wrapping the heterotic fivebrane around $K 3 \times T^{2}$. Obviously such instanton corrections cannot exist in $D>4$ dimensions. This fits with the observation that the prepotential of minimal five-dimensional supergravity is restricted to be purely cubic. Supersymmetry does not leave room for non-perturbative corrections and higher order $\alpha^{\prime}$ corrections. The only nonperturbative effects in five dimensions are discontinuous changes of the cubic coefficients $C_{I J K}$ at the boundaries of the Weyl chambers. This has been shown explicitly by considering the decompactification limit of the heterotic string 196]. In the M-theory language the discontinuous changes of the triple intersection numbers $C_{I J K}$ arise from flop transitions at boundaries of the Kähler cone 180].

There is at time no direct way to compute the instanton corrections to the four-dimensional heterotic prepotential. One can, however, compute them indirectly by using the duality between heterotic string theory on $K 3 \times T^{2}$ and type IIA string theory on a Calabi-Yau threefold 185 , 193. This duality can be motivated taking the six-dimensional duality between the heterotic string on $T^{4}$ and the IIA string on $K 3$ and expanding it adiabatically over a holomorphic twosphere $P^{1}$ [197. Globally the resulting geometries are taken to be fibrations rather than direct products. This way the compactification manifolds become $K 3 \times T^{2}$ on the heterotic side and a Calabi-Yau threefold on the type IIA side. The Calabi-Yau space has the special structure of

\footnotetext{
${ }^{11}$ In the literature one often defines an invariant dilaton, which contains both the perturbative string coupling and the $\theta$ angle. Note that there are several variants, called the 'invariant dilaton' or the 'quasi-invariant dilaton', which differ by conventional choices.
} 
a $K 3$-fibration, which means that it looks, locally and around a generic point, like a product of $P^{1}$ and $K 3$.

One can also directly show in four dimensions that the Calabi-Yau space of a IIA compactification must be a $K 3$ fibration in order to have a perturbative heterotic dual theory [198]. The basic observation is that the cubic part of the heterotic prepotential takes the special form

$$
\mathcal{F}^{\text {(het) }}\left(S, T^{a}\right)=-S T^{a} T^{b} \eta_{a b}-D_{a b c} T^{a} T^{b} T^{c}
$$

Comparing this to the cubic part of the IIA prepotential[2]

$$
\mathcal{F}^{(\mathrm{IIA})}=i \frac{1}{6} C_{A B C} z^{A} z^{B} z^{C}
$$

where $C_{A B C}$ are the triple intersection numbers it is obvious that the intersection form must take a special form and that the dilaton plays a distinguished role. It turns out that the heterotic dilaton corresponds to the IIA Kähler modulus that measures the size of the $P^{1}$ basis of the $K 3$ fibration. Since in the IIA theory all vector multiplet moduli are Kähler moduli, whereas the IIA dilaton sits in a hypermultiplet one can now compute the exact prepotential at tree level in IIA perturbation theory. The heterotic - IIA duality was therefore called a 'second quantized mirror map' in [193.

The weak coupling limit of the heterotic string, where heterotic perturbation theory is valid, corresponds to a particular large volume limit in the IIA moduli space, namely the limit of a large $P^{1}$. In order to ensure that the expansion for the IIA prepotential converges the other moduli have to be in the interior of the Kähler cone. Thus the perturbative heterotic limit corresponds to the IIA large volume limit. In this limit the prepotentials (and the higher derivative couplings) can be compared for concrete dual pairs. The particular family of heterotic theories with instanton numbers $(12+n, 12-n)$ is dual to a particular family of Calabi-Yau threefolds which can be constructed using methods of toric geometry [182, 183, 184]. These Calabi-Yau spaces are simultanously $K 3$-fibrations over $P^{1}$ and elliptic fibrations over the Hirzebruch surfaces $\mathbb{F}_{n}$. We remarked earlier that the three heterotic models with $n=0,1,2$ had the same cubic part of the prepotential and therefore were equivalent in the perturbative limit. At the non-perturbative level the models with $n=0,2$ turn out to live in the same moduli space, whereas the model with $n=1$ is a separate model [184.

One of the best and most studied examples for testing the duality is provided by the $(14,10)$ model [185, 189, 199]. The dual Calabi-Yau space to the $(14,10)$ model is a $K 3$-fibration which is also an elliptic fibration over the Hirzebruch surface $\mathbb{F}_{2}$. The model can be realized as a degree 24 hypersurface in weighted projective space with weights $(1,1,2,8,12)$. As the most simple test of the conjectured duality one might note that this Calabi-Yau space has $h_{1,1}=3$ and $h_{2,1}=243$, which, taking into account the universal hypermultiplet yields $N_{V}=3$ vector and $N_{H}=244$ hypermultiplets. The triple intersection form, in a suitable parametrization, yields the same cubic term in the prepotential as the heterotic one. The constant term in the prepotential is, on the IIA side proportional to $\zeta(3)$ times the Euler number, which is $2\left(h_{1,1}-h_{2,1}\right)=480$. This term also agrees with its heterotic counterpart. Moreover one can compare the next expansion coefficients of the heterotic and the IIA prepotential and finds that they agree.

Taking the duality for granted, the IIA tree level prepotential encodes the full nonperturbative physics of the heterotic theory. The resulting physics makes perfect sense and is a generalization of the Seiberg-Witten description [200] of $\mathcal{N}=2$ Super-Yang-Mills theory. Conversely one can recover the Seiberg-Witten prepotential of super Yang-Mills theory by decoupling gravity and the massive string modes [199]. Let us briefly indicate the picture that heterotic - type II duality provides for the dynamics of the heterotic string. In the full non-perturbative theory the structure and physical interpretation of the special loci in moduli space are different from those of the perturbative theory. In particular the monodromy matrices

\footnotetext{
${ }^{12}$ The relative factor $i$ is related to the different conventions for heterotic and type IIA moduli, that we explained above.
} 
corresponding to the singular loci now take the form

$$
\Gamma_{\text {nonpert }}=\left(\begin{array}{cc}
\mathbf{U} & \mathbf{Z} \\
\mathbf{W} & \mathbf{V}
\end{array}\right),
$$

which does not correspond to a symmetry of the perturbative Lagrangian. The physics associated to special loci in moduli space can be understood in terms of a dual local Lagrangian. To find the dual description one has to perform a symplectic transformation such that the particular monodromy one is interested in takes the form of a perturbative monodromy. This amounts to an electric - magnetic duality transformation, such that the new Lagrangian is weakly coupled in the vicinity of the special locus. One example is provided by the locus $T=U$, which in heterotic perturbation theory corresponds to non-abelian gauge symmetry enhancement. In the full theory the special locus is modified and the associated monodromies are of the nonperturbative type from the point of view of the heterotic Lagrangian. The physics can be best understood by going to the type IIB description by mirror symmetry [161. Then the special locus is part of the conifold locus, where the complex structure of the mirror Calabi-Yau degenerates. This degeneration corresponds to a vanishing three-cycle. The IIB theory has solitonic D3-branes, which can be wrapped holomorphically on the three-cycle to give pointlike BPS states from the four-dimensional point of view. As long as the three-cycle is large the BPS state is very heavy and corresponds to an extreme black hole of precisely the type we discuss in this paper. If, however, the three-cycle shrinks, the BPS state becomes very light and should be treated like an elementary particle. As usual for BPS black holes the correponding multiplet is a hypermultiplet and the charge carried by the state is magnetic or dyonic in terms of the heterotic basis for the charges. Thus, instead of massless charged vector multiplets one gets massless magnetic and dyonic hypermultiplets. This is obviously the generalization of the Seiberg-Witten solution of $\mathcal{N}=2 S U(2)$ super Yang-Mills theory to the case of local supersymmetry. There are various extensions of this to more complicated situations, which all have relations to field theory. We refer to [157, 201, 202 for review and references.

We already mentioned that the non-perturbative corrections change the topology of the moduli space. This results in what is called non-perturbative breaking of T-duality in [203, 158]. To understand what this means we recall that the original moduli space was the quotient space of a covering 'Teichmüller' space by the T-duality group. Our intuitive geometric notions are tied to the Teichmüller space: We imagine the radius of a dimension to be a number between 0 and $\infty$. Then the statement of T-duality is that small and big radii are 'actually the same' or 'physically indistinguishable'. But to describe inequivalent physics one can directly work with the moduli space, without reference to the Teichmüller space. If one decides to describe the physics of a compactification using the domain which contains the point $r=\infty$ then there is in principle no need to talk about radii smaller then the minimal one. The statement of non-perturbative T-duality breaking points out that it might happen, and in theories with 8 supercharges will generically happen, that the non-perturbative moduli space is not the quotient of a modified Teichmüller space by a modified T-duality group. Since the moduli space encodes all physics there is no actual problem with this. But we loose the intuitive interpretation based on the Teichmüller space.

We also note that at the non-perturbative level we still have discrete invariances. As we discussed above, T-duality transformations act on the perturbative prepotential as monodromies. At the non-perturbative level the monodromy group is modified but still exists, and one might consider the full mondromy group as the non-perturbative generalization of the T-duality group. In particular the monodromy group encodes exact discrete symmetries of the theory, like the exact $S-T$ exchange symmetries mentioned earlier. If the Calabi-Yau space is realized as an algebraic variety one can define a duality group $\Gamma$, which gets contributions from the monodromy group $\Gamma_{M}$ of the prepotential and from the group of symmetries of the defining the equations, $\Gamma_{W}$. In 204] it was argued that the duality group is given by the semidirect product $\Gamma_{M} \times \Gamma_{W}$. For one-moduli examples this is known to be true, see 187 for discussion and references. In comparison to perturbative heterotic T-duality groups it is interesting that in both cases braid 
groups seem to play a role. The relations between the heterotic tree level and perturbative T-duality group is given by replacing certain Weyl reflections by braidings 194, wheras in 205] it was shown that the duality group of a specific two-parameter Calabi-Yau moduli space is the central extension of a braid group.

\subsubsection{Higher Derivative Couplings in Heterotic Compactifications}

So far we only discussed the heterotic prepotential. In order to analyse the perturbative structure of the higher order couplings, we must look at their dependence on the string coupling $g_{S}$. We saw above that the perturbative Kähler potential is

$$
K\left(S, \bar{S}, T^{a}, \bar{T}^{a}\right)=\log g_{S}^{2}+\widehat{K}\left(T^{a}, \bar{T}^{a}\right) .
$$

Therefore the couplings take the form

$$
F^{(g)}(X)=-i m_{\text {String }}^{2-2 g} e^{(1-g) \widehat{K}} \mathcal{F}^{(g)}\left(S, T^{a}\right) .
$$

This time the dependence on the string coupling or dilaton is through the function $\mathcal{F}^{(g)}\left(S, T^{a}\right)$. Fortunately the dependence on $S$ is restricted by holomorphicity considerations as we discussed for the particular case of the prepotential. It can be linear, constant or of the form $e^{-S}$, corresponding to tree level, one loop and non-perturbative contributions. By explicit calculation one finds that the higher couplings $\mathcal{F}^{(g>1)}$ do not have a tree level term, whereas the tree level part of $\mathcal{F}^{(1)}$ is universal [206, 207]. In summary one finds the following structure:

$$
\begin{aligned}
\mathcal{F}^{(0)}\left(S, T^{a}\right) & =-S T^{a} T^{b} \eta_{a b}+h^{(0)}\left(T^{a}\right)+f^{(0)}\left(e^{-S}, T^{a}\right) \\
\mathcal{F}^{(1)}\left(S, T^{a}\right) & =24 S+h^{(1)}\left(T^{a}\right)+f^{(1)}\left(e^{-S}, T^{a}\right) \\
\mathcal{F}^{(g>1)}\left(S, T^{a}\right) & =h^{(g>1)}\left(T^{a}\right)+f^{(g>1)}\left(e^{-S}, T^{a}\right) .
\end{aligned}
$$

The prepotential was discussed at length in the preceeding paragraphs. Let us now look at the $C^{2}$-coupling $\mathcal{F}^{(1)}\left(S, T^{a}\right)$. For concreteness we consider the $(14,10) S T U$ model. Then 208, 191]

$$
\mathcal{F}^{(1)}(S, T, U)=24 S_{\mathrm{inv}}+\frac{b_{\mathrm{grav}}}{8 \pi^{2}} \log \eta^{-2}(i T) \eta^{-2}(i U)+\frac{1}{2 \pi^{2}} \log (j(i T)-j(i U)),
$$

where the invariant dilaton is defined by

$$
\begin{aligned}
S_{\mathrm{inv}}= & S-\frac{1}{2} \frac{\partial h^{(1)}(T, U)}{\partial T \partial U}-\frac{1}{8 \pi^{2}} \log (j(i T)-j(i U)) \\
= & S+p(T, U)+\frac{1}{8 \pi^{2}} \sum_{k, l \geq 0} k l c(k l) \mathrm{Li}_{1}\left(e^{-2 \pi(k T+l U)}\right) \\
& -\frac{1}{8 \pi^{2}} \mathrm{Li}_{1}\left(e^{-2 \pi(T-U)}\right)-\frac{1}{8 \pi^{2}} \log (j(i T)-j(i U)),
\end{aligned}
$$

where $p(T, U)$ is a linear polynomial, $c(n)$ are the modular coefficients defined in (5.38) and $\mathrm{Li}_{1}$ is the first polylogarithmic function, see appendix $\mathrm{E}$. The number $b_{\text {grav }}$ is the gravitational $\beta$-function coefficient, associated with the one-loop running of the $C^{2}$ term. Note that there is no constant term in $\mathcal{F}^{(1)}(S, T, U)$ and that we find again threshold corrections proportional to $\log (j(i T)-j(i U))$ which correspond to perturbative gauge symmetry enhancement.

The comparison of heterotic and type II $\mathcal{F}^{(1)}$-functions provides further evidence for heterotic - type II duality 206, 209, 210, 208, 191. One can in particular compare the expansion of the perturbative heterotic $\mathcal{F}^{(1)}\left(S, T^{a}\right)$ to that of its IIA counterpart in the large volume limit. The IIA function counts rational and elliptic curves inside the Calabi-Yau, whereas the heterotic function is related to expansion coefficients of modular forms. For the $(14,10)$ model 
a detailed comparison of the heterotic and IIA $\mathcal{F}^{(1)}$-functions has been performed in [191], with the result that both functions agree to the order one can compute.

The perturbative part of the higher couplings $\mathcal{F}^{(g>1)}\left(S, T^{a}\right)$ has also been computed in the $(14,10)$ model [207]. The resulting expressions are very complicated. They have no polynomial piece in the moduli, but start with a constant piece and include in their holomorphic part a sum over polylogs $\mathrm{Li}_{3-2 g}$, with coefficients related to modular forms. As far as a comparison is possible everything is consistent with heterotic - IIA duality. In particular the constant term is always related to the Euler number of the dual Calabi-Yau space with the correct $g$-dependent prefactor. The structure of the polylog terms fits with the counting of higher genus holomorphic curves in the dual Calabi-Yau.

Another way of testing heterotic - type II duality using the higher couplings $\mathcal{F}^{(g)}(z)$ is provided by the holomorphic anomaly [211, 212]. As in the case of type II theory, one has to distinguish between Wilsonian and physical couplings in the heterotic theory. The full physical couplings have a non-holomorphic part. In the perturbative heterotic theory the nonholomorphic pieces are necessary to make the couplings covariant with respect to symplectic transformations [111]. As we discussed in chapter 3 the holomorphic couplings are in general not symplectic functions. The physical couplings, however, have to transform as symplectic functions, and they have to be automorphic functions of the T-duality group. The lack of symplectic covariance of the holomorphic couplings is encoded in a symplectic anomaly equation [11], which coincides with the large radius limit of the type II holomorphic anomaly equation. For lower $g$ the anomaly equation together with other physical input fixes the non-holomorphic piece uniquely. This has been used to predict the non-holomorphic parts of the higher IIA couplings on the basis of heterotic T-duality in [212].

Finally we would like to stress that from the mathematical point of view heterotic - IIA duality predicts a deep relation between automorphic forms and Calabi-Yau geometry. As we saw the expansion coefficients of the couplings count holomorphic curves inside the Calabi-Yau manifold. The heterotic couplings are constrained by T-duality to be automorphic forms of the T-duality group and are related to infinite dimensional Lie algebras. Duality predicts that 'holomorphic curves are counted by (expansion coefficients of) automorphic forms'. This is a highly non-trivial statement, but confirmed by explicit computations.

\subsubsection{Compactifications with $\mathcal{N}=4$ Supersymmetry}

The main focus of this paper is on four-dimensional string vacua with $\mathcal{N}=2$ supersymmetry, but it is instructive to consider certain aspects of $\mathcal{N}=4$ compactifications in parallel. The most simple realization is provided by compactifying the heterotic string on a six-torus $T^{6}$. One can take any of the ten-dimensional gauge groups because the two theories are on the same moduli after compactification by virtue of T-duality.

For generic moduli the massless spectrum consists of $\mathcal{N}=4$ supergravity coupled to 22 abelian $\mathcal{N}=4$ vector multiplets. The gravity multiplet contains 6 graviphotons, resulting in a gauge group $U(1)^{28}$. The minimal terms in the Lagrangian are uniquely fixed by local $\mathcal{N}=4$ supersymmetry [213]. The moduli space is locally

$$
\mathcal{M}=\left.\frac{S U(1,1)}{U(1)}\right|_{S} \times\left.\frac{S O(6,22)}{S O(6) \times S O(22)}\right|_{T^{a}},
$$

where $S$ is the dilaton and $T^{a}$ are 132 geometric moduli which encode deformations of the metric of the torus, of the background $B$-field and of the Wilson lines. The global structure is determined by the combined S- and T-duality groups

$$
S L(2, \mathbb{Z})_{S} \times S O(6,22, \mathbb{Z})_{T}
$$

In contradistinction to the $\mathcal{N}=2$ case the two-derivative part of the Lagrangian is not corrected and the $\mathrm{S}$ - and T-duality group are symmetries of the full theory [214. As in the $\mathcal{N}=2$ case the theory contains a higher derivative gravitational $C^{2}$-term with a field dependent coupling 
$\mathcal{F}^{(1)}(S)$. At tree level this function is [206]

$$
\mathcal{F}^{(1)}(S)=24 S \text {. }
$$

There are no perturbative corrections to this result and in particular the gravitational $\beta$-function vanishes because the coefficient $b_{\text {grav }}$ vanishes, as a result of the matter content of the theory [208. But there has to be a non-perturbative correction, because the above coupling is not S-duality invariant [215. This is different for the two-derivative terms which are S-duality invariant at tree level and do not get any perturbative or non-perturbative correction. By looking for an S-duality invariant completion one finds

$$
\mathcal{F}^{(1)(\mathrm{hol})}(S)=\frac{24}{2 \pi i} \log \eta(4 \pi i S),
$$

which has the correct behaviour under S-duality and reduces to the tree level result in the large $\mathrm{S}$ limit. ${ }^{3}$ More precisely this is the holomorphic, Wilsonian piece of the coupling. We will discuss the full coupling in $\mathcal{N}=2$ language later.

The above reasoning was based on symmetry arguments. Using the $\mathcal{N}=4$ version of heterotic - IIA duality one can verify the result by explicit computation. The heterotic string on $T^{4}$ is equivalent to the IIA string on $K 3$ 80, 81, 216, 217 and after compactification to four dimensions the heterotic string on $T^{6}$ and the type II string on $K 3 \times T^{2}$ are on the same moduli. Since under the heterotic - type IIA map the heterotic dilaton is identified with the Kähler modulus of the $T^{2}$ one can compute the correction to $\mathcal{F}^{(1)}(S)$ in IIA perturbation theory by a one-loop calculation [215], which is similar to the heterotic $\mathcal{N}=2$ one-loop calculation that we discussed before. The corrections are elliptic $(g=1)$ world sheet instantons from the IIA perspective and they are non-perturbative instanton corrections for the heterotic string, which come from Euclidean fivebranes wrapped around the torus.

Let us now describe the $\mathcal{N}=4$ theory in the $\mathcal{N}=2$ language. The $\mathcal{N}=4$ gravity multiplet splits into various $\mathcal{N}=2$ multiplets: one gets the $\mathcal{N}=2$ gravity multiplet which contains one of the $\operatorname{six} \mathcal{N}=4$ graviphotons. Then one gets two $\mathcal{N}=2$ gravitini multiplets, which absorb four of the graviphotons. The remaining $\mathcal{N}=4$ graviphoton sits in a vector multiplet, together with the dilaton. The $22 \mathcal{N}=4$ vector multiplets decompose into $22 \mathcal{N}=2$ vector and 22 $\mathcal{N}=2$ hypermultiplets. A certain subsector of this, containing $\mathcal{N}=2$ supergravity coupled to $N_{V}=23$ vector multiplets, with moduli space

$$
\mathcal{M}=\frac{S U(1,1)}{U(1)} \times \frac{S O\left(2, N_{V}-1\right)}{S O(2) \times S O\left(N_{V}-1\right)},
$$

can be described using our $\mathcal{N}=2$ formalism. One should note that the prepotential and the higher couplings of the $\mathcal{N}=4$ and $\mathcal{N}=2$ theories differ beyond the tree level. For example the heterotic $\mathcal{N}=2$ function $\mathcal{F}^{(1)}\left(S, T^{a}\right)$ has corrections that depend on all the moduli and not only on $S$ and one does not expect S-duality. Nevertheless we can use our $\mathcal{N}=2$ formalism to get information about the $\mathcal{N}=4$ theory, and in particular about its black hole solutions, as we will see in the next chapter.

Finally there are special heterotic $\mathcal{N}=2$ compactifications which have non-renormalization properties similar to $\mathcal{N}=4$ models. These are stringy analogues of finite $\mathcal{N}=2$ gauge theories, where the matter content is chosen such that the $\beta$-function vanishes. The prime example of such a special heterotic $\mathcal{N}=2$ compactification is the so-called FHSV model [193], which has 12 vector and 11 hypermultiplets. The dual Calabi-Yau space has $h_{1,1}=11=h_{2,1}$ and therefore its Euler number vanishes, $\chi=0$. In this model the tree level moduli space is exact and S- and T-duality are non-perturbative symmetries. Like in the $\mathcal{N}=4$ case the $C^{2}$-coupling gets corrections beyond the tree level, and the corrections are precisely such that the function $\mathcal{F}^{(1)}\left(S, T^{a}\right)$ becomes invariant under the dualities 218. The coupling does not only depend on the dilaton but also on the other 10 moduli through an automorphic form of the T-duality group $O(2,10, \mathbb{Z})$. The algebraic structure behind the formula is controlled by the so-called 'faked Monster Lie superalgebra'.

\footnotetext{
${ }^{13}$ This form of $S$-dependence is also predicted by considering the so-called topological free energy 208 . The fact that such non-perturbative terms have to be present was pointed out in 215.
} 
102CHAPTER 5. FOURDIMENSIONAL STRING AND M-THEORY COMPACTIFICATIONS 


\section{Chapter 6}

\section{Four-Dimensional $\mathcal{N}=2$ Black Holes in String and M-Theory}

In this chapter we describe the computation of the black hole entropy for concrete models both from the macroscopic and the microscopic point of view. In the first section the macroscopic entropy is computed by solving the stabilization equations and plugging the result into the entropy formula (4.84). We start with black holes in type II string theory and M-theory. After discussing general properties of the entropy formula we consider the large radius limit of a general Calabi-Yau compactification. The corresponding $F$-function is leading order in $\alpha^{\prime}$ and receives contributions at tree and one-loop level in $g_{I I A}$. The one-loop term is linear in $C^{2}$. It turns out that we can also include a class of higher order terms, which can be computed in the M-theory picture 175, 176. For the most general black hole, with no restriction on the charges, we can bring the entropy to form (6.10). Fully explicit solutions are obtained after imposing the vanishing of some of the charges or reality constraints on the solution. We discuss three specific cases. The most important result is formula (6.22) for the macroscopic black hole entropy of the large volume limit of a Calabi-Yau compactification, with charge $p^{0}=0$. This is the most general case where the corresponding microscopic entropy is known from [219, 220].

Next we discuss black holes in heterotic $\mathcal{N}=2$ compactifications. In view of heterotic - IIA duality this is a special case of the discussion of type II black holes, but it deserves an explicit study for the following reason: In perturbative heterotic models T-duality is an exact symmetry and therefore one expects that the entropy formula is T-duality invariant. Moreover we can profit from the specific form of the heterotic prepotential and find solutions for the entropy without imposing constraints on the charges. We show in detail how one can make T-duality invariance manifest. In particular we show that the contribution of the perturbative prepotential to the entropy, including all $\alpha^{\prime}$-corrections, can be expressed in terms of the perturbative heterotic string coupling by formula (6.51). Next we include the higher couplings, but now restrict ourselves to the leading order in $\alpha^{\prime}$. In this case we find a manifestly T-dual formula by combining all terms into invariants of the T-duality group in formula (6.64). These two results extend the known results on the U-duality invariance of the entropy of $\mathcal{N}=8$ black holes 221 and on the T- and S-duality invariance of the entropy of $\mathcal{N}=4$ black holes 222. 223. We also discuss certain non-perturbative and non-holomorphic contributions to the entropy. This is illustrated by finding the manifestly T- and S-duality invariant formula (6.84) for the entropy of black holes in $\mathcal{N}=4$ compactifications in presence of higher curvature terms.

In the second part of the chapter we review the computation of the microscopic black hole entropy [219, 220]. Following 224] we discuss $\mathcal{N}=8$ and $\mathcal{N}=4$ compactifications on $T^{6}$ and $K 3 \times T^{2}$ in parallel with generic $\mathcal{N}=2$ compactifications on Calabi-Yau threefolds. The result is the microscopic entropy formula (6.97), which is valid for black holes with charge $p^{0}=0$ in the large radius limit of Calabi-Yau compactifications. This result agrees with the corresponding macroscopic entropy (6.22) and we discuss in detail why this is a highly nontrivial result, which on the macroscopic side depends crucially on the correct treatment of the 
higher curvature terms and on the use of the modified entropy formula (2.55) instead of the Bekenstein-Hawking area law.

\subsection{The Macroscopic Black Hole Entropy}

\subsubsection{Black Holes in Type II String Theory and in M-Theory}

We have already seen in chapter 1 that the stabilization equations (4.85) together with the model-independent entropy formula (4.84) fix the macroscopic entropy in terms of the electric and magnetic charges carried by the black hole. In order to give an explicit expression for the entropy one has to solve the stabilization equations for a specific choice of the function $F(X, \widehat{A})$. Due to the complicated nature of this function, explicit solutions can only be obtained for sufficiently simple choices of $F(X, \widehat{A})$ and for black holes which only carry a subset of the possible charges. Our presentation follows 224, where more details can be found. Results on the entropy of Calabi-Yau black holes in absence of higher curvature terms were obtained in 131, 225].

As a first step it is convenient to introduce rescaled $U(1)$-invariant variables by

$$
Y^{I}=e^{\mathcal{K} / 2} \bar{Z} X^{I} \text { and } \Upsilon=e^{\mathcal{K}} \bar{Z}^{2} \widehat{A} .
$$

Then the stabilization equations take the form

$$
\begin{aligned}
Y^{I}-\bar{Y}^{I} & =i p^{I} \\
F_{J}(Y, \Upsilon)-\bar{F}_{J}(\bar{Y}, \bar{\Upsilon}) & =i q_{J}
\end{aligned}
$$

where we used the homogenity properties of $F(X, \widehat{A})$. We also note that

$$
|Z|^{2}=p^{I} F_{I}(Y, \Upsilon)-q_{I} Y^{I}
$$

The model-independent entropy formula takes the form

$$
\mathcal{S}=\pi\left[|Z|^{2}+4 \operatorname{Im}\left(\Upsilon F_{\Upsilon}(Y, \Upsilon)\right)\right]
$$

where $\Upsilon=-64$. Before turning to explicit models we note that the charge dependence of the entropy formula follows a general pattern: Let $Q$ be a generic charge and recall that the function $F(Y, \Upsilon)$ has an expansion $F(Y, \Upsilon)=\sum_{g \geq 0} F^{(g)}(Y) \Upsilon^{g}$. Now suppose that we solve the stabilization equations iteratively in $\Upsilon$. To leading order, $Y^{I}$ and $F_{J}$ are proportional to $Q$ and the resulting entropy will therefore be proportional to $Q^{2}$, i.e. it is quadratic in the charges. By iteration we then find

$$
\mathcal{S}=\pi \sum_{g=0}^{\infty} a_{g} Q^{2-2 g},
$$

where the constant coefficient $a_{g}$ is related to the contribution of the function $F^{(g)}(Y)$.

We now turn to specific models. The most important model that we have to discuss is based on the function

$$
F(Y, \Upsilon)=D_{A B C} \frac{Y^{A} Y^{B} Y^{C}}{Y^{0}}+D_{A} \frac{Y^{A}}{Y^{0}} \Upsilon .
$$

This function describes the large volume limit of Calabi-Yau compactifications of IIA string theory or eleven-dimensional M-theory. It contains all terms which are (in type IIA language) tree-level in $\alpha^{\prime}$. The first and second term are tree level and one-loop in the type IIA coupling, respectively. The second term is related to a $C^{2}$-term of the form $D_{A} z^{A} C^{2}$ in the effective Lagrangian, where $z^{A}=Y^{A} / Y^{0}$. The coefficients $D_{A B C}, D_{A}$ are related to the triple intersection numbers and second Chern class number of the Calabi-Yau space by

$$
D_{A B C}=-\frac{1}{6} C_{A B C} \text { and } D_{A}=-\frac{1}{24} \frac{1}{64} c_{2 A} .
$$


In our discussion of the stabilization equation we will also include a function $G\left(Y^{0}, \Upsilon\right)$, which contains terms of higher order in $\Upsilon$,

$$
F(Y, \Upsilon)=D_{A B C} \frac{Y^{A} Y^{B} Y^{C}}{Y^{0}}+D_{A} \frac{Y^{A}}{Y^{0}} \Upsilon+G\left(Y^{0}, \Upsilon\right)
$$

where

$$
Y^{0} G_{0}+2 \Upsilon G_{\Upsilon}=2 G .
$$

The contributions encoded in $G\left(Y^{0}, \Upsilon\right)$ are in the M-theory picture due to loop and nonperturbative corrections of Kaluza-Klein modes of the graviton (D0-branes) around the Mtheory circle.

One now computes the explicit expression for $F_{0}$ and $F_{A}$ and gets formulae for $q_{0}$ and $q_{A}$ from the stabilization equations. Note that the function $G\left(Y^{0}, \Upsilon\right)$ only enters into the expression for $q_{0}$, because $G\left(Y^{0}, \Upsilon\right)$ does not depend on $Y^{A}$. The entropy takes the form?

$$
\mathcal{S}=\pi\left[|Z|^{2}-2 i D_{A}\left(\frac{Y^{A}}{\overline{Y^{0}}} \Upsilon-\frac{\bar{Y}^{A}}{Y^{0}} \bar{\Upsilon}\right)-2 i\left(\Upsilon G_{\Upsilon}-\bar{\Upsilon} G_{\bar{\Upsilon}}\right)\right]
$$

where $Z$ is given through the formula $|Z|^{2}=p^{I} F_{I}-q_{I} Y^{I}$ by

$$
\begin{aligned}
|Z|^{2}= & i D_{A B C}\left[\frac{3 Y^{A} Y^{B} \bar{Y}^{C}}{Y^{0}}-\frac{3 \bar{Y}^{A} \bar{Y}^{B} Y^{C}}{\bar{Y}^{0}}-\frac{Y^{A} Y^{B} Y^{C} \bar{Y}^{0}}{\left(Y^{0}\right)^{2}}+\frac{\bar{Y}^{A} \bar{Y}^{B} \bar{Y}^{C} Y^{0}}{\left(\bar{Y}^{0}\right)^{2}}\right] \\
& +i D_{A}\left[\frac{\bar{Y}^{A} \Upsilon}{Y^{0}}-\frac{Y^{A} \Upsilon}{\bar{Y}^{0}}-\frac{Y^{A} \bar{Y}^{0} \Upsilon}{\left(Y^{0}\right)^{2}}+\frac{\bar{Y}^{A} Y^{0} \bar{\Upsilon}}{\left(\bar{Y}^{0}\right)^{2}}\right] \\
& +\frac{1}{2} i\left(Y^{0}+\bar{Y}^{0}\right)\left(G_{0}-\bar{G}_{0}\right)+\frac{1}{2} p^{0}\left(G_{0}+\bar{G}_{0}\right) .
\end{aligned}
$$

Explicit solutions can be found only in cases where one does not take the most general configuration of charges. The three cases where solutions are available are (i) $p^{0}=0$, (ii) so-called axion-free black holes where the moduli $z^{A}=Y^{A} / Y^{0}$ are purely imaginary and (iii) black holes with $\operatorname{Re} Y^{0}=0$.

Black Holes with $p^{0}=0$

When setting $p^{0}=0$ it imediately follows that $Y^{0}$ is real, $Y^{0}=\bar{Y}^{0}$. Then the stabilization equation involving $q_{A}$ simplifies and one can derive

$$
Y^{A}=\frac{1}{6} Y^{0} D^{A B} q_{B}+\frac{1}{2} i p^{A}
$$

where we defined $D^{A B}$ by

$$
D_{A B}:=D_{A B C} p^{C} \text { and } D_{A B} D^{B C}=\delta_{A}^{C} .
$$

Using the $q_{0}$ stabilization equation one finds

$$
4\left(Y^{0}\right)^{2}=\frac{D_{A B C} p^{A} p^{B} p^{C}-4 D_{A} p^{A} \Upsilon}{\widehat{q}_{0}+i\left(G_{0}-\bar{G}_{0}\right)},
$$

where $\widehat{q}_{0} \equiv q_{0}+\frac{1}{12} D^{A B} q_{A} q_{B}$. Furthermore one determines

$$
|Z|^{2}=-\frac{D_{A B C} p^{A} p^{B} p^{C}-2 D_{A} p^{A} \Upsilon}{Y^{0}}+i Y^{0}\left(G_{0}-\bar{G}_{0}\right),
$$

and the entropy is given by

$$
\mathcal{S}=-4 \pi Y^{0} \widehat{q}_{0}-i \pi\left(3 Y^{0} G_{0}+2 \Upsilon G_{\Upsilon}-\text { h.c. }\right) .
$$

\footnotetext{
${ }^{1}$ We do not make use of the fact that $\Upsilon$ is real on the horizon, because the formula looks more symmetric in terms of $\Upsilon$ and $\bar{\Upsilon}$.
} 
One can now solve (6.14) iteratively for $Y^{0}$ in order to express everything in terms of the charges. First note that one already has an explicit expression if $G\left(Y^{0}, \Upsilon\right)=0$. We denote this value by $y^{0}$ :

$$
y^{0}=\frac{1}{2} \sqrt{\frac{D_{A B C} p^{A} p^{B} p^{C}-4 D_{A} p^{A} \Upsilon}{\widehat{q}_{0}}} .
$$

The corresponding zeroth order value for $Y^{A}$ is $y^{A}=\frac{1}{6} D^{A B} q_{B}+\frac{i}{2} p^{A}$ so that the moduli $z^{A}=y^{A} / y^{0}$ are

$$
z^{A}=i p^{A} \sqrt{\frac{\widehat{q}_{0}}{D_{A B C} p^{A} p^{B} p^{C}-4 D_{A} p^{A} \Upsilon}}+\frac{1}{6} D^{A B} q_{B} .
$$

Next observe that we have to restrict the charges to be either positive or negative in order to make sure that the right hand side of (6.14) is positive. We take the magnetic charges $p^{A}$ to be positive. Then $D_{A B C} p^{A} p^{B} p^{C}=-\frac{1}{6} C_{A B C} p^{A} p^{B} p^{C}$ and $-4 D_{A} p^{A} \Upsilon=-\frac{1}{6} c_{2 A} p^{A}$ are negative and we have to take $\widehat{q}_{0}$ to be negative, $\widehat{q}_{0}<0$. The fact that we have to specify the signs of the charges reflects that the Kähler moduli space is a cone. With the above choice the square root in (6.18) is real and $z^{A}$ has a positive imaginary part. This means that the physical range of parameters is $\operatorname{Im} z^{A}>0$ and that $\operatorname{Im} z^{A}=0$ is the boundary of the Kähler cone.

We have to make sure that the large volume approximation is valid near the horizon. In the type IIA picture where the vector multiplet moduli are Kähler moduli this is the case when the moduli near the horizon take values deep inside the Kähler cone, $\operatorname{Im} z^{A}>>0$. This is achieved by taking $\left|\widehat{q}_{0}\right|$ to be much larger than the other charges: $\left|\widehat{q}_{0}\right|>>\left|p^{A}\right|$. Next we have to make sure that the curvature at the horizon is small so that the higher curvature terms can be treated as small corrections. This can be done by making all charges large, so that we have to impose

$$
\left|\widehat{q}_{0}\right|>>\left|p^{A}\right|>>0
$$

on the charges.

Finally we have to impose that the function $G\left(Y^{0}, \Upsilon\right)$ can be treated as a small perturbation, so that an iterative solution of (6.14) makes sense. This imposes the constraint $\left|\operatorname{Im}\left(G_{0}\left(y^{0}, \Upsilon\right)\right)\right|<<\left|\widehat{q}_{0}\right|$.

The first iterative step then yields

$$
Y^{0}=y^{0}\left(1+\frac{1}{2} \frac{i\left(G_{0}\left(y^{0}, \Upsilon\right)-\bar{G}_{0}\left(\bar{y}^{0}, \bar{\Upsilon}\right)\right)}{\left|\widehat{q}_{0}\right|}+\cdots\right) .
$$

Inserting this into (6.16) gives the entropy formula

$$
\mathcal{S}=2 \pi \sqrt{\left|\widehat{q}_{0}\right|\left(-\frac{1}{6} D_{A B C} p^{A} p^{B} p^{C}-256 D_{A} p^{A}\right)}-2 \pi i\left(G\left(y^{0}, \Upsilon\right)-\bar{G}\left(\bar{y}^{0}, \bar{\Upsilon}\right)\right)+\cdots
$$

The zeroth order approximation, which corresponds to the tree order in $\alpha^{\prime}$ is given by setting $G\left(Y^{0}, \Upsilon\right)=0$. When expressing the coefficients $D_{A B C}, D_{A}$ in terms of the topological quantities $C_{A B C}, c_{2 A}$ the entropy takes the form

$$
\mathcal{S}=2 \pi \sqrt{\frac{1}{6}\left|\widehat{q}_{0}\right|\left(C_{A B C} p^{A} p^{B} p^{C}+c_{2 A} p^{A}\right)} .
$$

This formula agrees with a microscopic entropy formula derived from state counting, as we will see in section 6.2.2.

\section{Axion-Free Black Holes}

We now turn to axion-free black holes, which are characterized by the condition that the moduli $z^{A}=Y^{A} / Y^{0}$ are imaginary. Using $\bar{Y}^{0} Y^{A}+Y^{0} \bar{Y}^{A}=0$, it follows that

$$
Y^{A}=i p^{A} \frac{Y^{0}}{\lambda}, \quad \text { and } \quad Y^{0}=\frac{1}{2}\left(\lambda+i p^{0}\right),
$$


where $\lambda$ is real constant. The charges $q_{A}$ are given by

$$
q_{A}=-3 D_{A B C} p^{B} p^{C} \frac{p^{0}}{\lambda^{2}}-4 D_{A} \frac{p^{0} \Upsilon}{\lambda^{2}+\left(p^{0}\right)^{2}} .
$$

Therefore the charges are tightly constrained. We have found a quadratic equation for $\lambda$, which fixes all the moduli in terms of the charges. The stabilization equation for $q_{0}$ shows that $q_{0}$ is not independent, but is given by

$$
q_{A} p^{A}+3 q_{0} p^{0}=-D_{A} p^{A} \Upsilon \frac{16 p^{0}}{\lambda^{2}+\left(p^{0}\right)^{2}}-3 i p^{0}\left(G_{0}-\bar{G}_{0}\right) .
$$

In this case we get $|Z|$ from (6.11)

$$
\begin{aligned}
|Z|^{2}= & -2 D_{A B C} p^{A} p^{B} p^{C} \frac{\lambda^{2}+\left(p^{0}\right)^{2}}{\lambda^{3}}+4 D_{A} p^{A} \Upsilon \frac{\lambda^{2}-\left(p^{0}\right)^{2}}{\lambda\left(\lambda^{2}+\left(p^{0}\right)^{2}\right)} \\
& +\frac{1}{2} i \lambda\left(G_{0}-\bar{G}_{0}\right)+\frac{1}{2} p^{0}\left(G_{0}+\bar{G}_{0}\right),
\end{aligned}
$$

and the entropy is

$$
\begin{aligned}
\mathcal{S}=\pi[ & -2 D_{A B C} p^{A} p^{B} p^{C} \frac{\lambda^{2}+\left(p^{0}\right)^{2}}{\lambda^{3}}+8 D_{A} p^{A} \Upsilon \frac{\lambda}{\lambda^{2}+\left(p^{0}\right)^{2}} \\
& \left.+\frac{1}{2} i \lambda\left(G_{0}-\bar{G}_{0}\right)+\frac{1}{2} p^{0}\left(G_{0}+\bar{G}_{0}\right)-2 i \Upsilon\left(G_{\Upsilon}-\bar{G}_{\Upsilon}\right)\right] .
\end{aligned}
$$

For large $Y^{0}$ the leading part of $G$ is $G=i c\left(Y^{0}\right)^{2}$, with $c$ real 175. Taking $G=i c\left(Y^{0}\right)^{2}$ and $D_{A}=0$ the result (6.27) of [224] reduces to the one obtained in [131].

\section{Black Holes with $\operatorname{Re} Y^{0}=0$}

The third case where we can have an explicit solution is $\operatorname{Re} Y^{0}=0$ which implies $Y^{0}=\frac{1}{2} i p^{0}$. The stabilization equations imply

$$
\begin{aligned}
p^{0} q_{A} & =-6 D_{A B C}\left(Y^{B} Y^{C}+\bar{Y}^{B} \bar{Y}^{C}\right)-4 D_{A} \Upsilon \\
\left(p^{0}\right)^{2} q_{0} & =4 D_{A B C} p^{A}\left(Y^{B} Y^{C}+Y^{B} \bar{Y}^{C}+\bar{Y}^{B} \bar{Y}^{C}\right)+4 D_{A} p^{A} \Upsilon-i\left(p^{0}\right)^{2}\left(G_{0}-\bar{G}_{0}\right) .
\end{aligned}
$$

Again the charge $q_{0}$ is not independent. The constraint is

$$
p^{0} p^{I} q_{I}=2 D_{A B C} p^{A} p^{B} p^{C}-i\left(p^{0}\right)^{2}\left(G_{0}-\bar{G}_{0}\right) .
$$

From (6.11) and (6.10) we obtain

$$
\begin{aligned}
|Z|^{2} & =\frac{2}{p^{0}} D_{A B C}(Y+\bar{Y})^{A}(Y+\bar{Y})^{B}(Y+\bar{Y})^{C}+\frac{4}{p^{0}} D_{A}(Y+\bar{Y})^{A} \Upsilon+\frac{1}{2} p^{0}\left(G_{0}+\bar{G}_{0}\right), \\
\mathcal{S} & =\frac{2 \pi}{p^{0}} D_{A B C}(Y+\bar{Y})^{A}(Y+\bar{Y})^{B}(Y+\bar{Y})^{C}-2 i \pi(G-\bar{G}),
\end{aligned}
$$

where we made use of the homogenity property $(6.9)$ for $G$.

The equations (6.28) are quadratic equations for the scalars $Y^{A}$ and can be used to express them in terms of the charges. However, we do not wish to pursue this in full generality. Below we will determine the value of the $Y^{A}$ for type-II models with a dual heterotic description.

We conclude our discussion of IIA black holes with a remark on the dependence of the entropy on the topology of the Calabi-Yau space. When considering all contributions to the couplings that arise at tree level and loop level in $\alpha^{\prime}$ we find that the topological quantities involved are the intersection numbers, the second Chern class and the Euler number. The entropy depends on these model dependent data and on the charges

$$
\mathcal{S}=\mathcal{S}\left(p^{I}, q_{J} \mid C_{A B C}, c_{2 A}, \chi\right) .
$$

It is amusing to note that the topological quantities $C_{A B C}, c_{2 A}, \chi$ are necessary and sufficient, according to Wall's theorem [226], to determine the underlying Calabi-Yau space up to homotopy. When world-sheet instantons are taken into account, then the entropy also depends on finer data, namely the world-sheet instanton number. 


\subsubsection{Black Holes in Heterotic String Theory}

We now turn to the discussion of black holes in $\mathcal{N}=2$ heterotic string compactifications. As a consequence of heterotic - type IIA duality this is, technically, a special case of the IIA string compactifications, where the Calabi-Yau space is restricted to be a $K 3$ fibration. But concerning the physics involved, heterotic black holes are worth to be studied in their own right.

\section{Tree Level Black Holes}

We start our investigation by considering black hole solutions based on the tree-level prepotential

$$
F=-\frac{Y^{1} Y^{a} \eta_{a b} Y^{b}}{Y^{0}},
$$

where

$$
Y^{a} \eta_{a b} Y^{b}=Y^{2} Y^{3}-\sum_{i}\left(Y^{3+i}\right)^{2} .
$$

We use the conventions of 224]. The results of this subsection were obtained in 227, 228].

The conventions for heterotic models are explained in chapter 5 . The physical moduli are $S=-i Y^{1} / Y^{0}$ and $T^{a}=-i Y^{1+a} / Y^{0}$, where $T^{a}=T, U, V^{i}$. A special case of this class is the $S T U$-model, where

$$
F=-\frac{Y^{1} Y^{2} Y^{3}}{Y^{0}} .
$$

Heterotic tree level black holes have the special property that one can solve the stabilization equations explicitly without restricting the choice of the non-vanishing charges. In order to show that the resulting entropy is manifestly T-duality invariant, and in the case of the $S T U$-model even is triality invariant, we introduce certain invariants of the T-duality group $O\left(2, N_{V}-1, \mathbb{Z}\right)$. First recall that the heterotic charges $\left(N^{I}, M_{J}\right)$ (the charges associated with the symplectic basis $P^{I}, Q_{J}$ adapted to heterotic perturbation theory) differ from the IIA charges $p^{I}, q_{J}$ (the charges associated with the symplectic section $Y^{I}, F_{J}$ determined by the prepotential) by a symplectic transformation:

$$
\begin{aligned}
\left(N^{I}\right) & =\left(p^{0}, q_{1}, p^{2}, \ldots\right) \\
\left(M_{J}\right) & =\left(q_{0},-p^{1}, q_{2}, \ldots\right)
\end{aligned}
$$

T-duality acts on the section $\left(P^{I}, Q_{J}\right)$ by a specific subset of the 'classical' symplectic transformations introduced in chapter 5 . The action on the symplectic vector of charges is read off from the expression for the BPS-mass, which has to be symplectically invariant:

$$
M_{B P S}^{2}=N_{I} Q^{I}-M^{I} P_{I},
$$

where the $\left(U(1)\right.$ invariant) section $\left(Q^{I}, P_{J}\right)$ is evaluated at spatial infinity. This implies that under the transformation

$$
\left(\begin{array}{c}
Q^{I} \\
P_{J}
\end{array}\right) \rightarrow \Gamma\left(\begin{array}{c}
Q^{I} \\
P_{J}
\end{array}\right)
$$

the charges transform as

$$
\left(N^{I}, M_{J}\right) \rightarrow\left(N^{I}, M_{J}\right) \Gamma^{T} .
$$

For a classical T-duality transformation this imples

$$
\left(N^{I}\right) \rightarrow\left(N^{I}\right) \mathbf{U}^{T} \text { and }\left(M_{J}\right) \rightarrow\left(M_{J}\right) \mathbf{U}^{-1},
$$


where $\mathbf{U} \in O\left(2, N_{V}-1, \mathbb{Z}\right)$. This gives rise to three obvious invariants;

$$
\begin{aligned}
\langle M, M\rangle & =2\left(M_{0} M_{1}+\frac{1}{4} M_{a} \eta^{a b} M_{b}\right)=2\left(-q_{0} p^{1}+\frac{1}{4} q_{a} \eta^{a b} q_{b}\right) \\
\langle N, N\rangle & =2\left(N^{0} N^{1}+N^{a} \eta_{a b} N^{b}\right)=2\left(p^{0} q_{1}+p^{a} \eta_{a b} p^{b}\right) \\
M \cdot N & =M_{I} N^{I}=q_{0} p^{0}-q_{1} p^{1}+q_{2} p^{2}+\cdots+q_{n} p^{n} .
\end{aligned}
$$

Here $\eta^{a b}$ is the inverse matrix of $\eta_{a b}$ (5.17):

$$
\left(\eta^{a b}\right)=\left(\begin{array}{ccc}
0 & 2 & 0 \\
2 & 0 & 0 \\
0 & 0 & -\delta^{i j}
\end{array}\right) .
$$

The first two invariants use the pseudo-orthogonality of $\mathbf{U}$, whereas the third invariant is based on the fact that $M$ and $N$ transform with contragradient matrices.

One can now use the stabilization equations to establish

$$
\begin{aligned}
Y^{a} & =\frac{1}{S+\bar{S}}\left[-\frac{1}{2} \eta^{a b} q_{b}-i \bar{S} p^{a}\right] \\
|Z|^{2} & =(S+\bar{S})\left(\bar{Y}^{a} \eta_{a b} Y^{b}-\frac{\bar{Y}^{0}}{Y^{0}} Y^{a} \eta_{a b} Y^{b}+\text { h.c. }\right) \\
q_{1} p^{0} & =-\left(\frac{\bar{Y}^{0}}{Y^{0}}-1\right) Y^{a} \eta_{a b} Y^{b}+\text { h.c. }
\end{aligned}
$$

These equations can be combined into

$$
|Z|^{2}=\frac{S+\bar{S}}{2}\langle N, N\rangle,
$$

which implies (since no higher derivative terms are considered)

$$
\mathcal{S}=\frac{\pi}{2}(S+\bar{S})\langle N, N\rangle .
$$

This relation is very interesting, because it relates the entropy to the tree level string coupling evaluated on the event horizon

$$
\mathcal{S}=\left.\langle N, N\rangle \frac{4 \pi^{2}}{g_{S}^{2}}\right|_{\text {Horizon }} .
$$

We will see that this relation generalizes to the full perturbative level, where one cannot, in general, compute the moduli, the dilaton and the entropy as functions of the charges.

In the tree level case one can use the remaining stabilization equations to express the dilaton explicitly in terms of charges

$$
S=i \frac{M \cdot N}{\langle N, N\rangle}+\sqrt{\frac{\langle M, M\rangle}{\langle N, N\rangle}-\frac{(M \cdot N)^{2}}{\langle N, N\rangle^{2}}}
$$

and therefore the entropy is

$$
\mathcal{S}=\pi \sqrt{\langle M, M\rangle\langle N, N\rangle-(M \cdot N)^{2}} .
$$

This formula is manifestly T-duality invariant. For the special case of the $S T U$-model one can verify that the entropy is invariant under the full triality group. If more moduli are present, the formula is still invariant under S-duality. 
As we discussed in chapter 5 we can describe a subsector of the $\mathcal{N}=4$ heterotic compactification in terms of the tree level $\mathcal{N}=2$ theory. The full $\mathcal{N}=4$ theory contains four additional gauge fields and therefore there are four electric and four magnetic charges that one has to switch off. The full $\mathcal{N}=4$ entropy formula [222, 223] is obtained from our tree level $\mathcal{N}=2$ formula by replacing the $O\left(2, N_{V}-1, \mathbb{Z}\right)$ invariants by the corresponding $O(6,24, \mathbb{Z})$ invariants.

Furthermore for specific choices of the charges, like purely electric or magnetic black holes, and, more generally, for black holes where $M^{I}$ and $N_{I}$ are parallel as vectors, one finds that one gets a vanishing entropy. The cases of purely electric or magnetic charges correspond to singular solutions of the stabilization equations, where the moduli take singular values either on the horizon or at infinity. The associated black hole solutions are degenerate at the horizon, which signals that the solution does not make sense as a four-dimensional geometry. This can be understood from the $\mathcal{N}=4$ point of view as follows: In $\mathcal{N}=4$ theories there are two types of BPS states, short and intermediate ones. But only black holes that are members of intermediate multiplets and which have 4 Killing spinors correspond to regular solutions of the stabilization equations, whereas black holes that are in short $\mathcal{N}=4$ BPS multiplets and which have 8 Killing spinors necessarily are degenerate. One can show that charge configurations with parallel magnetic and electric charge vectors correspond to short multiplets whereas the more generic charge configurations with non-parallel charge vectors correspond to intermediate multiplets.

\section{Perturbative Heterotic Black Holes}

Let us now consider black hole entropy on the basis of the full perturbative heterotic prepotential 131, 229. Remember that the perturbative prepotential has the form

$$
\mathcal{F}=-\frac{Y^{1} Y^{a} \eta_{a b} Y^{b}}{Y^{0}}+\left(Y^{0}\right)^{2} h\left(T^{a}\right)
$$

where the one-loop correction $h\left(T^{a}\right)$ does not depend on the dilaton S.

The action of the T-duality group $O\left(2, N_{V}-1, \mathbb{Z}\right)$ on the heterotic section now takes the form

$$
\Gamma_{\text {pert }}=\left(\begin{array}{cc}
\mathbf{U} & \mathbb{O} \\
\mathbf{U}^{T,-1} \Lambda & \mathbf{U}^{T,-1}
\end{array}\right),
$$

where the symmetric matrix $\Lambda$ accounts for the perturbative modification. The charges now transform in the following way under T-duality:

$$
\left(N^{I}\right) \rightarrow\left(N^{I}\right) \mathbf{U}^{T} \text { and }\left(M_{J}\right) \rightarrow\left(M_{J}\right) \mathbf{U}^{-1}+\left(N^{I}\right) \Lambda^{T} \mathbf{U}^{-1}
$$

Note that the transformation law of the electric charges is modified whereas the magnetic charges $N^{I}$ still transform as at tree level. Therefore $\langle N, N\rangle$ is still an invariant, whereas $\langle M, M\rangle$ and $M \cdot N$ are not.

Proceeding as in the tree level case one can show that the entropy is given by

$$
\mathcal{S}=\left.\langle N, N\rangle \frac{4 \pi^{2}}{g_{\text {pert }}^{2}}\right|_{\text {Horizon }},
$$

where $g_{\text {pert }}$ is the perturbative string coupling defined by

$$
\frac{4 \pi}{g_{\text {pert }}^{2}}=\frac{1}{2}\left(S+\bar{S}+V_{G S}\left(T^{a}, \bar{T}^{a}\right)\right) .
$$

Since the perturbative coupling is by construction T-duality invariant, we have shown that the entropy is T-duality invariant, irrespective of the precise form of $h\left(T^{a}\right)$.

Having derived this important general result we now turn to explicit examples. Beyond tree level we can only get explicit results for the entropy in terms of the charges if the function $h\left(T^{a}\right)$ 
is sufficiently simple and if we restrict the charges. Let us first consider the $S T U$-model and include the cubic part of the one loop correction, which is tree level in $\alpha^{\prime}$. Then the prepotential is

$$
F=-\frac{Y^{1} Y^{2} Y^{3}+\frac{1}{3}\left(Y^{3}\right)^{3}}{Y^{0}} .
$$

The coefficients of this cubic polynomial are now proportional to the triple intersection form of the dual Calabi-Yau space. The solution for the entropy is a special case of the discussion given for IIA black holes, where we discussed three different configurations of charges that allow explicit solutions. Here we only display the formula for non-axionic black holes with $p^{0}=q_{A}=0$. One finds

$$
\mathcal{S}=2 \pi \sqrt{\left|q_{0}\right|\left(p^{1} p^{2} p^{3}+\frac{1}{3}\left(p^{3}\right)^{3}\right)} .
$$

Note that the $\left(p^{3}\right)^{3}$ term is due to the one-loop contribution to the heterotic prepotential, i.e. to the two-derivative part of the action. This is the most simple example of a quantum effect that modifies the entropy of a black hole. From the IIA perspective the prepotential is purely classical, whereas the higher derivative couplings are loop effects.

With some more effort one can treat the $\alpha^{\prime}$-loop correction to the $S T U$ prepotential exactly. More generally we can consider axion-free black holes in a theory with prepotential

$$
F=D_{A B C} \frac{Y^{A} Y^{B} Y^{C}}{Y^{0}}+i c\left(Y^{0}\right)^{2}
$$

where the coefficients $D_{A B C}$ are arbitrary and need not correspond to a perturbative heterotic model. The constant $c$ is chosen to be real because its imaginary part could be removed by a symplectic transformation. In IIA models $D_{A B C}$ is proportional to the triple intersection numbers whereas $c$ is proportional to the Euler number. The $S T U$ model has

$$
D_{A B C}=-\frac{1}{6} \varepsilon_{A B C}-\frac{1}{3} \delta_{A 3} \delta_{B 3} \delta_{C 3} \text { and } c=\frac{\chi \zeta(3)}{16 \pi^{3}},
$$

where the Euler number of the dual Calabi-Yau space is $2\left(h_{1,1}-h_{2,1}\right)=2 \cdot(3-243)=-480$.

Using the stabilization equations and proceeding like in the previous IIA discussion of nonaxionic black holes we find that the entropy is given by

$$
\mathcal{S}=-2 \pi\left(q_{0}-2 c \lambda\right)\left[\lambda+\frac{\left(p^{0}\right)^{2}}{\lambda}\right],
$$

where $\lambda$ is now a solution of the cubic equation

$$
D_{A B C} p^{A} p^{B} p^{C}+2 c \lambda^{3}=q_{0} \lambda^{2} .
$$

This equation can be solved explicitly, but the resulting expressions are not very illuminating, and therefore we will not write them down. One can explicitly verify that the corrections due to the constant terms are small in the large volume limit, by expanding the solution of the full cubic equation in terms of the approximate solution with $c=0$. In the general non-axionic case only the charges $q_{0}, p^{0}, p^{A}$ are independent whereas the $q_{A}$ are determined by

$$
q_{A}=-9 \frac{p^{0}}{\lambda^{2}} D_{A B C} p^{B} p^{C} .
$$

This can be understood in terms of the underlying reality constraint that restricts the moduli to purely real values (in the heterotic parametrization by $S, T^{a}$ ). Since there are $2 N_{V}+2$ charges but only $2 N_{V}$ real moduli, one has $N_{V}$ real constraints, leaving $N_{V}+2$ independent charges $q_{0}, p^{0}, p^{A}$.

If one imposes in addition $p^{0}=0$ it follows that $q_{A}=0$ and the nonvanishing changes are $q_{0}, p^{A} \cdot{ }^{2}$

One can also discuss black hole solutions based on the same prepotential with the constraint Re $Y^{0}$. In this case there is no dependence on $c$ and we get nothing new compared to the previous discussion.

\footnotetext{
${ }^{2}$ When solving the stabilization equations for the explicit form of the entropy and of the moduli one has to discuss this case separately, because one cannot divide by $p^{0}=0$.
} 


\section{World-Sheet Instanton Corrections to the Heterotic Black Hole Entropy}

One might wonder whether it is possible to derive explicit results when the world sheet instanton corrections are taken into account. It turns out that one can get concrete results when expanding the prepotential around one of the special loci, where a particular contribution to $h\left(T^{a}\right)$ is

dominant. This was discussed in 230] for the STU and in [231] for the ST model with the result that one gets corrections to the entropy that depend logarithmically on the charges, but in such a way that the entropy is invariant under monodromy transformations. One sees explicitly that the entropy, in the IIA language, depends on the rational instanton numbers.

One other point about higher $\alpha^{\prime}$-corrections is noteworthy. The higher $\alpha^{\prime}$-corrections have the particular feature that they introduce transcendental numbers such as $\zeta(3)$ into the entropy formula. It is difficult to imagine how such numbers could be understood in terms of counting internal excitations. In 230, 231] a microscopic model for the $\alpha^{\prime}$-loop contribution was proposed in terms of a gas of membranes. But it was remarked in 133 that special values of the $\zeta$-function such as $\zeta(3)$ are related to the world sheet instanton coefficients. Thus it might be that one has to take into account all such terms simultanously and that the full result then yields a rational contribution. In conclusion the issue of $\alpha^{\prime}$-corrections is not yet properly understood and our later discussion of the microscopic entropy will exclusively concern contributions at $\alpha^{\prime}$-tree level.

\section{Higher Curvature Contributions to the Heterotic Black Hole Entropy}

So far our discussion of heterotic black holes was based on the prepotential. We will now consider the effect of higher curvature corrections [224]. Again, this is technically a special case of our discussion of IIA black holes, but interesting regarding the physical content. In particular the heterotic higher coupling functions are modular forms and we expect that the entropy is T-duality invariant.

Let us first consider a general heterotic model at tree level in the string coupling, which is defined by the function

$$
F(Y, \Upsilon)=-\frac{Y^{1} Y^{a} \eta_{a b} Y^{b}}{Y^{0}}+c_{1} \frac{Y^{1}}{Y^{0}} \Upsilon
$$

Here

$$
Y^{a} \eta_{a b} Y^{b}=Y^{2} Y^{3}-\sum_{a=4}^{n}\left(Y^{a}\right)^{2}, \quad a=2, \ldots, n,
$$

with real constants $\eta_{a b}$ and $c_{1}$. The calculation goes through as in the case $\Upsilon=0$, with minor modifications. Using the stabilization equations we first derive

$$
\mathcal{S}=\frac{1}{2} \pi(S+\bar{S})\left(\langle N, N\rangle-512 c_{1}\right)
$$

The value of the dilaton at the horizon is found to be

$$
S=\sqrt{\frac{\langle M, M\rangle\langle N, N\rangle-(M \cdot N)^{2}}{\langle N, N\rangle\left(\langle N, N\rangle-512 c_{1}\right)}}+i \frac{M \cdot N}{\langle N, N\rangle},
$$

and the entropy therefore is

$$
\mathcal{S}=\pi \sqrt{\langle M, M\rangle\langle N, N\rangle-(M \cdot N)^{2}} \sqrt{1-\frac{512 c_{1}}{\langle N, N\rangle}},
$$

where we used the classical T-duality invariants. These formulae reduce properly to the ones derived from the tree level prepotential. Moreover they are manifestly T-duality invariant. As 
in the case of the heterotic tree level black holes we can regard this as the truncation of an $\mathcal{N}=4$ model. Conversely we can lift our $\mathcal{N}=2$ result to the full $\mathcal{N}=4$ theory by replacing $O\left(2, N_{V}-1, \mathbb{Z}\right)$ by the T-duality group $O(6,22, \mathbb{Z})$ of a heterotic $\mathcal{N}=4$ compactification. We will return to $\mathcal{N}=4$ compactifications in the next section.

The tree level result can now be extended to more general models with higher curvature corrections in the same way as we did in the IIA case. Let us then continue the discussion of black holes with $\operatorname{Re} Y^{0}=0$, that we started when considering IIA black holes. We will restrict ourselves to type-II models with a dual heterotic description,

$$
D_{A B C} Y^{A} Y^{B} Y^{C}=-Y^{1} Y^{a} \eta_{a b} Y^{b}, \quad D_{A} Y^{A}=c_{1} Y^{1},
$$

where $D_{1 a b}=-\frac{1}{3} \eta_{a b}$. The first equation in (6.28) yields

$$
\begin{aligned}
Y^{a} & =\frac{1}{Y^{1}+\bar{Y}^{1}}\left[\frac{1}{4} p^{0} \eta^{a b} q_{b}+i \bar{Y}^{1} p^{a}\right] \\
4 c_{1} \Upsilon+p^{0} q_{1}+p^{a} \eta_{a b} p^{b} & =\frac{1}{\left(Y^{1}+\bar{Y}^{1}\right)^{2}}\left[\frac{1}{4}\left(p^{0}\right)^{2} q_{a} \eta^{a b} q_{b}+\left(p^{1}\right)^{2} p^{a} \eta_{a b} p^{b}+p^{0} p^{1} p^{a} q_{a}\right] .
\end{aligned}
$$

Substituting this into the above entropy formula, we obtain

$$
\begin{aligned}
\mathcal{S}= & -\frac{2 \pi}{p^{0}\left(Y^{1}+\bar{Y}^{1}\right)}\left[\frac{1}{4}\left(p^{0}\right)^{2} q_{a} \eta^{a b} q_{b}+\left(p^{1}\right)^{2} p^{a} \eta_{a b} p^{b}+p^{0} p^{1} p^{a} q_{a}\right]-2 i \pi(G-\bar{G}) \\
= & -\frac{\pi}{p^{0}} \sqrt{\left(\left(p^{0}\right)^{2} q_{a} \eta^{a b} q_{b}+4\left(p^{1}\right)^{2} p^{a} \eta_{a b} p^{b}+4 p^{0} p^{1} q_{a} p^{a}\right)\left(q_{1} p^{0}+p^{a} \eta_{a b} p^{b}+4 c_{1} \Upsilon\right)} \\
& -2 i \pi(G-\bar{G}) .
\end{aligned}
$$

We thus see that we have to choose $p^{0}<0$. This expression can be rewritten as follows in terms of the heterotic electric and magnetic charges $M_{I}$ and $N^{I}$,

$$
\begin{aligned}
\mathcal{S}= & -\frac{\pi}{p^{0}} \sqrt{\left(\left(p^{0}\right)^{2}\langle M, M\rangle+\left(p^{1}\right)^{2}\langle N, N\rangle+2 p^{0} p^{1} M \cdot N\right)\left(\langle N, N\rangle+8 c_{1} \Upsilon\right)} \\
& -2 i \pi(G-\bar{G}) .
\end{aligned}
$$

Observe that the charges are subject to the constraint (6.29), which in the case at hand reads $p^{0} M \cdot N+p^{1}\langle N, N\rangle=-i\left(p^{0}\right)^{2}\left(G_{0}-\bar{G}_{0}\right)$. Substituting this into (6.68) yields

$$
\begin{aligned}
\mathcal{S}= & \pi \sqrt{\langle M, M\rangle\langle N, N\rangle-(M \cdot N)^{2}-\left(p^{0}\right)^{2}\left(G_{0}-\bar{G}_{0}\right)^{2}} \sqrt{1-\frac{512 c_{1}}{\langle N, N\rangle}} \\
& -2 i \pi(G-\bar{G}),
\end{aligned}
$$

where we also used $\Upsilon=-64$. This expression reduces to (6.64) in the case of $G=0$.

\section{Non-Perturbative and Non-Holomorphic Contributions to the Heterotic Black Hole Entropy}

We already remarked that tree level $\mathcal{N}=2$ heterotic black holes can be considered as special cases of tree level $\mathcal{N}=4$ black holes. We now return to this subject and discuss non-perturbative aspects of such black holes [224]. This provides an example where the entropy is modified through non-holomorphic contributions to the gravitational couplings.

First recall that in $\mathcal{N}=4$ models (and in $(\mathcal{N}=4$ )- like $\mathcal{N}=2$ models such as the FHSV model) the minimal terms in the Lagrangian are exact, whereas the $C^{2}$-coupling $F^{(1)}(Y)$ is modified by non-perturbative corrections. The reason is that the dilaton dependence of $F^{1}(Y)$ is given by

$$
\mathcal{F}^{(1)}(S)=24 S,
$$

which is not invariant under S-duality. Therefore the tree level result has to be modified by non-perturbative contributions such that the full function $F^{(1)}(S)$ is compatible with S-duality. 
Let us discuss this in the $\mathcal{N}=2$ language by considering a function of the form

$$
F(Y, \Upsilon)=-\frac{Y^{1} Y^{a} \eta_{a b} Y^{b}}{Y^{0}}+F^{(1)}(S) \Upsilon
$$

The discussion is most easily done in the heterotic basis $\left(Y^{0}, F_{1}, Y^{2}, \ldots, F_{0},-Y^{1}, F_{2}, \ldots\right)$. As a first step one can verify that the invariance under the T-duality group $O\left(2, N_{V}-1, \mathbb{Z}\right)$ holds irrespective of the form of $F^{(1)}(S)$. (See [224] for a more detailed account.)

Next one studies S-duality transformations

$$
S \rightarrow \frac{a S-i b}{i c S+d} .
$$

Such a transformation is induced by the following symplectic transformation of the section:

$$
\begin{aligned}
Y^{0} & \rightarrow \tilde{Y}^{0}=d Y^{0}+c Y^{1}, & F_{0} & \rightarrow \widetilde{F}_{0}=a F_{0}-b F_{1}, \\
Y^{1} & \rightarrow \tilde{Y}^{1}=a Y^{1}+b Y^{0}, & F_{1} & \rightarrow \widetilde{F}_{1}=d F_{1}-c F_{0}, \\
Y^{a} & \rightarrow \tilde{Y}^{a}=d Y^{a}-\frac{1}{2} c \eta^{a b} F_{b}, & F_{a} & \rightarrow \widetilde{F}_{a}=a F_{a}-2 b \eta_{a b} Y^{b} .
\end{aligned}
$$

The corresponding action on the charges is

$$
M_{I} \rightarrow \widetilde{M}_{I}=a M_{I}-2 b \eta_{I J} N^{J}, \quad N^{I} \rightarrow \widetilde{N}^{I}=d N^{I}-\frac{1}{2} c \eta^{I J} M_{J},
$$

implying that the T-duality invariant combinations of charges transform under S-duality according to

$$
\begin{aligned}
\langle M, M\rangle & \rightarrow a^{2}\langle M, M\rangle+b^{2}\langle N, N\rangle-2 a b M \cdot N, \\
\langle N, N\rangle & \rightarrow c^{2}\langle M, M\rangle+d^{2}\langle N, N\rangle-2 c d M \cdot N, \\
M \cdot N & \rightarrow-a c\langle M, M\rangle-b d\langle N, N\rangle+(a d+b c) M \cdot N .
\end{aligned}
$$

We have now to take into account that the $F_{I}$ are not independent objects, but functions of $S$ and the moduli. Therefore the transformation behaviour (6.73) of the $F_{I}$ has to be induced by the transformations of the $Y^{I}$. The crucial observation is that the S-duality transformation (6.72) does in general not induce the correct transformation behaviour of the $F_{I}$, but that the correct transformation follows if $f(S)=-i \partial F^{(1)} / \partial S$ is a modular function of weight 2 ,

$$
f(S) \rightarrow(i c S+d)^{2} f(S) .
$$

It is well known that the tree level function $F^{(1)}(S)=i c_{1} S$ indeed receives corrections that are positive powers of $e^{-S}$ that are not visible in perturbation theory and can complete $F^{(1)}(S)$ to a covariant object 215]. In $\mathcal{N}=2$ models (including the FHSV model) there are also moduli dependent corrections [218], but our focus here is S-duality in $\mathcal{N}=4$ models, where such corrections are absent. Therefore the function $F^{(1)}(S)$ does not depend on geometric moduli. The full function $F^{(1)}(S)$ cannot be holomorphic and satisfy property (6.76) at the same time, because there are no (holomorphic) modular forms of weight 2 . There are, however, non-holomorphic objects with the desired transformation behaviour. We arrive at the same conclusion as in the discussion of the T-duality properties of higher couplings: The physical couplings, which are invariant under all symmetries of the theory differ by non-holomorphic terms from the Wilsonian couplings, which are holomorphic, but do not necessarily have all symmetries.

Therefore we have to replace the holomorphic function $F^{(1)}(S)$ by a non-holomorphic function $F^{(1)}(S, \bar{S})$. A concrete example of a non-holomorphic, but covariant function is provided by

$$
F^{(1)}(S, \bar{S})=-i c_{1} \frac{6}{\pi}\left(\log \eta^{2}(S)+\log (S+\bar{S})\right),
$$

where $\eta(S)$ is the Dedekind $\eta$-function. This function reduces to $i c_{1} S$ in the limit $S \rightarrow \infty$, whereas

$$
f(S, \bar{S})=-i \partial_{S} F^{(1)}(S, \bar{S})=c_{1} \frac{3}{\pi^{2}} G_{2}(S, \bar{S})
$$


is a non-holomorphic modular form of weight two. Here

$$
G_{2}(S, \bar{S})=G_{2}(S)-\frac{2 \pi}{S+\bar{S}}
$$

is the non-holomorphic, but modular covariant second Eisenstein series, whereas

$$
G_{2}(S)=-4 \pi \partial_{S} \log \eta(S)
$$

is the holomorphic but non-covariant second Eisenstein series. Note that the function $F^{(1)}(S, \bar{S})$ is not strictly invariant but transforms as

$$
F^{(1)}(S, \bar{S}) \rightarrow F^{(1)}(S, \bar{S})+i c_{1} \frac{6}{\pi} \log (-i c \bar{S}+d) .
$$

This does not change the gravitational coupling but acts as a graviational $\theta$-shift. Also note that above we ignored possible anti-holomorphic contributions, i.e. terms holomorphic in $\bar{S}$, which vanish in the perturbative limit. In comparison to our discussion in chapter 5 we have identified the non-holomorphic part of $F^{(1)}(S, \bar{S})$ by symmetry arguments.

One can now analyse the stabilization equations based on the function

$$
F(Y, \Upsilon)=-\frac{Y^{1} Y^{a} \eta_{a b} Y^{b}}{Y^{0}}+F^{(1)}(S, \bar{S}) \Upsilon
$$

The resulting

$$
|Z|^{2}=\frac{\left(M_{I}+2 i S \eta_{I K} N^{K}\right) \eta^{I J}\left(M_{J}-2 i \bar{S} \eta_{J L} N^{L}\right)}{2(S+\bar{S})}
$$

is manifestly S- and T-duality invariant. When computing the entropy one finds that it is not invariant under S-duality. This indicates that besides the non-holomorphic contribution to the prepotential there must be an additional non-holomorphic contribution to the physical $C^{2}$ coupling. S-duality is restored if the physical $C^{2}$ coupling is given by $F^{(1)}(S, \bar{S})+i c_{1} \frac{3}{\pi} \log (S+\bar{S})$. Then the manifestly $\mathrm{S}$ - and T-duality invariant formula for the entropy is

$$
\begin{aligned}
\mathcal{S} & =\pi\left[|Z|^{2}+4 \operatorname{Im}\left(\Upsilon F^{(1)}(S, \bar{S})+i c_{1} \frac{3}{\pi} \Upsilon \log (S+\bar{S})\right)\right] \\
& =\frac{\pi}{2} \frac{\left(M_{I}+2 i S \eta_{I K} N^{K}\right) \eta^{I J}\left(M_{J}-2 i \bar{S} \eta_{J L} N^{L}\right)}{S+\bar{S}}+768 c_{1} \log \left[(S+\bar{S})|\eta(S)|^{4}\right]
\end{aligned}
$$

This formula depends implicitly on the function $f(S, \bar{S})$ through the equation

$$
f(S, \bar{S}) \Upsilon=\frac{1}{4} \frac{1}{(S+\bar{S})^{2}}\left(M_{I}-2 i \bar{S} \eta_{I K} N^{K}\right) \eta^{I J}\left(M_{J}-2 i \bar{S} \eta_{J L} N^{L}\right)
$$

which determines $S$ in terms of the charges. This equation can be solved iteratively in $\Upsilon$. In the case of a purely imaginary dilaton, the tree level solution $S=i M \cdot N /\langle N, N\rangle$ is exact.

\subsection{The Microscopic Black Hole Entropy}

\subsubsection{Computation of the Microscopic Black Hole Entropy}

In this section we will review the derivation of the microscopic black hole entropy. The microscopic picture of a four-dimensional black hole is obtained by embedding it into ten-dimensional string theory or eleven-dimensional M-theory. These higher dimensional theories have $p$-brane solitons, which can upon compactification reduce to four-dimensional black holes. Type II $p$-branes with Ramond-Ramond charge provide the low energy description of the type II Dbranes that one can introduce in string perturbation theory 11]. Therefore $p$-branes with

Ramond-Ramond charges are themselves called D-branes or Dp-branes for simplicity. The pbrane solitons of eleven-dimensional supergravity are called Mp-branes. The correspondence 
between D-branes and Ramond-Ramond charged p-branes can be used to compute the microscopic entropy of five- and four-dimensional extremal black holes. Starting from the work of [12, which introduced the method and for the first time achieved quantitative agreement between macroscopic and microscopic black hole entropy, this has been extended to five- and four-dimensional black holes in compactifications with $\mathcal{N}=8,4,2$ supersymmetry. The D-brane model also allows to study the entropy of near-extremal black holes, of Hawking radiation and of greybody factors. We refer to [13] for a review and references. The microscopic entropy of four-dimensional $\mathcal{N}=2$ black holes was computed in [219, 220] 3 ]

A $p$-brane is a solution of the effective supergravity equations of motion, which is translationally invariant along $p$ space directions and behaves in the transversal directions like a charged black hole. We refer to [233] for an extensive review. There is an extremal limit where the tension of a $p$-brane equals its charge(density), and in this limit the solution has Killing spinors and is a BPS state of the underlying supersymmetry algebra. Such extremal $p$-branes are higher-dimensional generalizations of the extremal Reissner-Nordstrøm black hole. As usual for BPS states one can have multi-centered solutions, which describe static configurations of extremal $p$-branes located at arbitrary positions.

In order to describe black holes of $\mathcal{N}=2$ supergravity in terms of $p$-branes of type II string theory or M-theory, one has to compactify $p$-branes on Calabi-Yau manifolds. Since this is complicated we first illustrate the structure of the configurations in the context of toroidal compactifications of type II or M-theory. It turns out that the toroidal compactification of a single $p$-brane yields a four-dimensional black hole with vanishing event horizon. This singularity is a compactification artefact which signals that close to the horizon the solution does not make sense as a four-dimensional geometry. In order to describe four-dimensional black holes with a finite event horizon one has to use more complicated $p$-brane bound states. Here one makes use of the fact that specific combinations of $p$-branes (or other BPS solitons) still have Killing spinors and therefore are themselves BPS states. Four-dimensional black holes are obtained by combining four different species of BPS solitons. Here $p$-branes are refered to as different species if they yield different charges by compactification. This is the case when they have different values of $p$, or if they are wraped on homologically different cycles. In order to have a finite horizon the configuration must have precisely four Killing spinors, i.e. it breaks $7 / 8$ of the 32 supersymmetries of the higher-dimensional vacuum.

We will now give two explicit examples which have generalizations to Calabi-Yau compactifications. The first one is a IIA configuration of three D4-branes with charges $p^{1}, p^{2}, p^{3}$ and $q_{0}$ D0-branes. The geometry is such that the $D 4$ branes are wraped on the three different fourcycles of the internal six-torus, such that they mutually intersect transversely on two-cycles and triple-intersect over a zero-cycle. The corresponding ten-dimensional string frame metric is 234

$$
\begin{aligned}
d s_{10}^{2}= & \frac{-1}{\sqrt{H_{0} H^{1} H^{2} H^{3}}} d t^{2}+\sqrt{H_{0} H^{1} H^{2} H^{3}} d \mathbf{x}^{2} \\
& +\sqrt{\frac{H_{0} H^{1}}{H^{2} H^{3}}}\left(d y_{1}^{2}+d y_{2}^{2}\right)+\sqrt{\frac{H_{0} H^{2}}{H^{1} H^{3}}}\left(d y_{3}^{2}+d y_{4}^{2}\right)+\sqrt{\frac{H_{0} H^{3}}{H^{1} H^{2}}}\left(d y_{5}^{2}+d y_{6}^{2}\right)
\end{aligned}
$$

where $y_{i}$ are coordinates along the torus directions and $x_{m}$ are coordinates along the three space directions. The functions $H_{0}, H^{A}$ only depend on the radius $r=\sqrt{x_{1}^{2}+x_{2}^{2}+x_{3}^{3}}$ and are harmonic functions. By toroidal compactification one obtains an extremal four-dimensional black hole with charges $q_{0}, p^{A}$ and metric

$$
d s_{4}^{2}=\frac{-1}{\sqrt{H_{0} H^{1} H^{2} H^{3}}} d t^{2}+\sqrt{H_{0} H^{1} H^{2} H^{3}} d \mathbf{x}^{2} .
$$

The charges are related to the harmonic function as in chapter 4 String and Einstein frame

\footnotetext{
${ }^{3}$ The releyant brane configurations and the microscopic interpretation of the leading term were already discussed in 232, 13
} 
coincide because the four-dimensional IIA dilaton is constant for this solution. We refer to 147 for a more detailed discussion.

From the M-theory point of view $D 0$ branes are Kaluza-Klein modes of the elevendimensional supergravity multiplet along the M-circle, whereas D4 branes result from wraping one dimension of the M5-brane on the M-circle. This explains the structure of the M-theory configuration that describes the same four-dimensional black hole from the eleven-dimensional point of view. One takes a configuration of three M5-branes with charges $p^{A}$ which mutually intersect transversely on three cycles and triple-intersect over a string (one-cycle). One now has to compactify on the M-circle and on a six-torus. The configuration is wraped such that the string wraps on the $\mathrm{M}$-circle. If the radius of the M-circle is taken to be much larger then the six-torus, the configuration describes a five-dimensional black string. In order to account for the D0 branes of the corresponding IIA configuration one has to put $\left|q_{0}\right|$ quanta of lightlike, left-moving (for definiteness) momentum on the string. In four dimensions one obtains the same black hole as before with charges $q_{0}, p^{A}$. The corresponding eleven-dimensional metric is 235]

$$
\begin{aligned}
d s_{11}^{2}= & \frac{1}{\left(H^{1} H^{2} H^{3}\right)^{1 / 3}}\left(d u d v+H_{0} d u^{2}+H^{1} H^{2} H^{3} d \mathbf{x}^{2}\right. \\
& \left.+H^{1}\left(d y_{1}^{2}+d y_{2}^{2}\right)+H^{2}\left(d y_{3}^{2}+d y_{4}^{2}\right)+H^{3}\left(d y_{5}^{2}+d y_{6}^{2}\right)\right),
\end{aligned}
$$

where $u, v$ are light cone coordinates involving time and the M-circle direction.

The above pattern of compactification generalizes to compactifications on $K 3 \times T^{2}$ and on Calabi-Yau threefolds, and then describes extremal black holes of $\mathcal{N}=4$ and $\mathcal{N}=2$ supergravity, respectively. Like the six-torus $T^{6}$ these manifolds posess non-trivial four-cycles on which the above brane configurations can be wraped, and the four-cycles intersect over two-cycles and triple-intersect over zero-cycles. A configuration with charges $p^{A}$ corresponds to wraping the $\mathrm{D} 4$ or M5 branes on a four-cycle $\mathcal{P}$ in the homology class $p^{A}\left[\Sigma_{A}\right]$, where $\Sigma_{A}$ is a basis of $H_{4}(X, \mathbb{Z})$. Note that $A$ now runs over the number of homology generators, which equals the Betti-number $b_{2}(X)=b_{4}(X)$, where $X$ is either a Calabi-Yau threefold, $K 3 \times T^{2}$ or $T^{6}$. The four-cycle has to be holomorphic in order that the configuration is a BPS state 236. The validity of both the macroscopic black hole solutions and the state counting require that we are working in a limit where both $\alpha^{\prime}$-corrections and space-time loops are suppressed. This implies that one has to take all the charges to be large, and $\left|q_{0}\right|$ must be much larger than all the other charges. Thus, one has to impose the same hierarchy of charges both from the macroscopic and the microscopic point of view:

$$
\left|q_{0}\right|>>p^{A}>>0 \text {. }
$$

The fact that the $p^{A}$ are large implies that $\mathcal{P}$ is, in the language of algebraic geometry, a very ample divisor of $X$. Technically this means that $X$ can be embedded into a higher dimensional projective space, such that $\mathcal{P}$ is a hyperplane section. Very ample divisors have various nice properties that have to be used for the state counting.

The divisor $\mathcal{P}$ is not a rigid object but can be holomorphically deformed inside $X$. For very ample divisors a generically chosen representative of such a family is always smooth and therefore is a compact complex Kähler surface. The state counting will be done for such smooth choices of $\mathcal{P}$. The concrete cycle that we described in the context of toroidal compactification is not smooth, because it is a sum of cycles which mutually intersect. At the intersections the cycle is not a manifold. But if the charges $p^{A}$ are sufficiently big, it is guaranteed that every generic deformation of this configuration is smooth. The adaequate picture for the state counting is that one wraps a single D4- or M5-brane on a smooth complex Kähler surface of the homology type specified by the charges.

The state counting has been performed both in the M-theory and in the type IIA picture [219, 220]. We will review the M-theory analysis of [219] in the following and we treat the cases of $T^{6}, K 3 \times T^{2}$ and Calabi-Yau threefolds in parallel. A more detailed presentation can be found in [224]. The M-theory analysis starts with the world volume theory of the M5-brane. The zero 
modes of the M5-brane fit into a tensor multiplet of six-dimensional $(0,2)$ supersymmetry. One takes the radius of the $\mathrm{M}$-circle to be much larger than the radius of the Calabi-Yau manifold and compactifies the M5-brane theory on the divisor $\mathcal{P}$. The resulting two-dimensional effective theory is a superconformal theory with $(0,4)$ supersymmetry. As discussed above the black hole does not correspond to the ground state of this theory but to an excited BPS state where one has switched on $\left|q_{0}\right|$ quanta of left-moving momentum. In the limit of very large $\left|q_{0}\right|$ the asymptotic density of states $d$ and the corresponding microscopic entropy are given by Cardy's formula

$$
d \simeq \exp \left(\mathcal{S}_{\text {micro }}\right) \simeq \exp \left(2 \pi \sqrt{\frac{1}{6}\left|q_{0}\right| c_{L}}\right),
$$

where $c_{L}$ is the left-moving central charge of the two-dimensional effective M5-brane theory. Thus the entropy is determined by counting in how many ways the $\left|q_{0}\right|$ quanta of momentum can be distributed among the zero mode excitations of the M5-brane. The central charge $c_{L}$ encodes how many distinct degrees of freedom exist. This number has to be determined by counting the zero modes of the compactification of the M5-brane theory on $\mathcal{P}$.

The six-dimensional $(0,2)$ tensor multiplet contains a selfdual antisymmetric tensor, five scalars, which describe the motion of the M5 brane along the five transverse directions and two Weyl fermions. Since $\mathcal{P}$ is a Kähler manifold one can express the numbers of the corresponding two-dimensional zero modes in terms of the independent Hodge numbers of $\mathcal{P}$, which are $h_{1,0}(\mathcal{P}), h_{2,0}(\mathcal{P})$ and $h_{1,1}(\mathcal{P})$. The numbers $b_{2}^{ \pm}(\mathcal{P})$ of selfdual and antiselfdual harmonic twoforms are given in terms of Hodge numbers by $b_{2}^{+}(\mathcal{P})=2 h_{2,0}(\mathcal{P})+1$ and $b_{2}^{-}(\mathcal{P})=h_{1,1}(\mathcal{P})-1$.

By dimensional reduction of the antisymmetric tensor one gets $b_{2}^{-}(\mathcal{P})$ left-moving and $b_{2}^{+}(\mathcal{P})$ right-moving scalars. Three of the five directions transverse to the M5-brane are non-compact, whereas the other two directions lie inside the Calabi-Yau space. The zero modes associated with these directions correspond to deformations of $\mathcal{P}$ inside $X$, i.e. they are sections of the normal bundle of $\mathcal{P}$. For a very ample divisor the number of these sections can be counted by a Riemann-Roch theorem, with the result that one gets $2 h_{2,0}(\mathcal{P})-2 h_{1,0}(\mathcal{P})$ left- and right-moving scalars. Finally the compactification of the 2 six-dimensional Weyl spinors yields left- and right-moving two-dimensional fermions that are counted by the sums of the odd and even Betti numbers, respectively. In summary one gets the following numbers of massless two-dimensional fields:

$$
\begin{aligned}
N_{\text {bosonic }}^{\text {left }} & =2 h_{2,0}(\mathcal{P})+h_{1,1}(\mathcal{P})+2-2 h_{1,0}(\mathcal{P}), \\
N_{\text {bosonic }}^{\text {right }} & =4 h_{2,0}(\mathcal{P})+4-2 h_{1,0}(\mathcal{P}), \\
N_{\text {fert }}^{\text {fertionic }} & =4 h_{1,0}(\mathcal{P}), \\
N_{\text {fermionic }}^{\text {right }} & =4 h_{2,0}(\mathcal{P})+4,
\end{aligned}
$$

where we used $h_{0,0}(\mathcal{P})=1$.

The next step is to express the Hodge numbers of $\mathcal{P}$ in terms of topological data of $X$ and the homology of $\mathcal{P}$. The topological data are the triple intersection form $C_{A B C}$, the components $c_{2 A}$ of the second Chern class of $X$ and the Hodge number $h_{1,0}(X)$. The homology class of $\mathcal{P}$ is given by the charges $p^{A}$. Then, the Hodge numbers of $\mathcal{P}$ are given by

$$
\begin{aligned}
& h_{2,0}(\mathcal{P})=\frac{1}{6} C_{A B C} p^{A} p^{B} p^{C}+\frac{1}{12} c_{2 A} p^{A}+h_{1,0}(\mathcal{P})-1, \\
& h_{1,1}(\mathcal{P})=\frac{2}{3} C_{A B C} p^{A} p^{B} p^{C}+\frac{5}{6} c_{2 A} p^{A}+2 h_{1,0}(\mathcal{P}) \\
& h_{1,0}(\mathcal{P})=h_{1,0}(X) .
\end{aligned}
$$

The first two lines follow from the index theorems for the Euler number and Hirzebruch signature, whereas the last line is the Lefshetz hyperplane theorem applied to a very ample divisor.

We can now write down the left- and right-moving central charges $c_{L / R}=N_{\text {bosonic }}^{\text {left }}+$ $\frac{1}{2} N_{\text {fermionic }}^{\text {left }}$ :

$$
c_{L}=C_{A B C} p^{A} p^{B} p^{C}+c_{2 A} p^{A}+4 h_{1,0}(X)
$$




$$
c_{R}=C_{A B C} p^{A} p^{B} p^{C}+\frac{1}{2} c_{2 A} p^{A}+4 h_{1,0}(X) .
$$

The corresponding entropy is

$$
\mathcal{S}_{\text {micro }}=2 \pi \sqrt{\frac{1}{6}\left|q_{0}\right|\left(C_{A B C} p^{A} p^{B} p^{C}+c_{2 A} p^{A}+4 h_{1,0}(X)\right)} .
$$

This is not yet our final result, due to a subtlety that concerns the cases $K 3 \times T^{2}$ and $T^{6}$, but is irrelevant for Calabi-Yau threefolds. As we explained above the two-dimensional conformal field theory has to be a $(0,4)$ supersymmetric sigma-model, so that our configuration describes a BPS state. This requires that the number of right-moving bosons and fermions has to match. Moreover the right-moving scalars must parametrize a quaternionic manifold and therefore the number of right-moving real scalars should be a multiple of four. If $X$ is a Calabi-Yau threefold, then $h_{1,0}(X)=0$ and the above constraints are satisfied. This is, however, not the case for $K 3 \times T^{2}$, which has $h_{1,0}(X)=1$ and for $T^{6}$ which has $h_{1,0}(X)=3$. Therefore our mode counting is not consistent with supersymmetry. Note however that these two cases differ from the case of a Calabi-Yau threefold in that one also gets $h_{1,0}(X)$ gauge fields from the dimensional reduction of the six-dimensional tensor field. These two-dimensional gauge fields are not dynamical and therefore do not contribute to the entropy as degrees of freedom. But they might modify the counting of the other modes, provided that these modes are charged. If we assume this, then the number of left- and right-moving scalars is reduced by $2 h_{1,0}(\mathcal{P})$ due to gauge symmetry. In order to get a supersymmetric right-moving spectrum the same mechanism must remove $4 h_{1,0}(\mathcal{P})$ right-moving fermions. If one furthermore assumes that this mechanism is left-right symmetric, then all left-moving $4 h_{1,0}(\mathcal{P})$ fermions are removed. As a result the entropy formula takes the form

$$
\mathcal{S}_{\text {micro }}=2 \pi \sqrt{\frac{1}{6}\left|q_{0}\right|\left(C_{A B C} p^{A} p^{B} p^{C}+c_{2 A} p^{A}\right)} .
$$

Note that this formula also applies to Calabi-Yau threefolds, where $h_{1,0}(\mathcal{P})=0$.

Besides two-dimensional supersymmetry we can invoke two further independent arguments in favour of the modified state counting for $K 3 \times T^{2}$ and $T^{6}$. The first is microscopic in nature. Anomaly inflow arguments can be used to determine the numbers $c_{L}$ and $c_{R}$ and they yield the same values as our modified state counting [237]. The second argument comes from comparison with the macroscopic black hole entropy. As we showed in the beginning of this chapter the entropy must be an even function of the charges, implying that $c_{L}$ must be odd in the charges. An entropy formula of the type (6.94) with $h_{1,0}(X) \neq 0$ is therefore not compatible with a macroscopic entropy formual based on low energy supergravity.

Finally we note that one can generalize the above discussion from black holes with charges $q_{0}, p^{A}$ to the more general case where only $p^{0}=0$, while the charges $q_{A}$ are non-vanishing. This can be done by adding an M2-brane to the configuration, which is wraped on a two-cycle in the homology class $q_{A} \Sigma^{A}$, where the $\Sigma^{A}$ form a basis of $H_{2}(X, \mathbb{Z}),[13$. As discussed in [13, 219] this has the effect that $q_{0}$ is replaced by

$$
\widehat{q_{0}}=q_{0}+\frac{1}{12} D^{A B} q_{A} q_{B}
$$

in the entropy formula, where $D^{A B}$ is the inverse of $D_{A B}=D_{A B C} p^{C}$. Thus the final entropy formula, which is valid for black holes with $p^{0}=0$ at tree level in $\alpha^{\prime}$ is

$$
\mathcal{S}_{\text {micro }}=2 \pi \sqrt{\frac{1}{6}\left|\widehat{q}_{0}\right|\left(C_{A B C} p^{A} p^{B} p^{C}+c_{2 A} p^{A}\right)} .
$$

\subsubsection{Comparison of Macroscopic and Microscopic Results}

Comparing the formulae (6.22) and (6.97) we find that the macroscopic and microscopic entropies agree. This is a highly non-trivial test of the microscopic picture of black holes provided by string theory in view of the complications we had to go through in order to derive these 
formulae. On the macroscopic side we had in particular to include the higher curvature terms using an elaborate formalism and we had to take into account the modifications of the area law. It is illustrative to look at certain points in more detail. The first subtle point is that the model-independent entropy formula (4.84) which was derived by substituting our near-horizon solution into Wald's formula turned out to be symplectically covariant. Despite the fact that the Lagrangian contains several terms involving the Riemann tensor we only got one correction term to the area law, and this term involved the function $F_{\widehat{A}}$, which is a symplectic function, in contradistinction to almost all other expressions that one can form out of $F(X, \widehat{A})$. All noncovariant contributions to Wald's formula either cancel or vanish for the near-horizon solution. This illustrates that the generalized entropy formula makes perfect sense in supersymmetric theories.

Our second remark concerns the explicit form of the two terms in the entropy formula. It is very crucial that the higher curvature terms enter in two different ways: First they explicitly modify the near horizon solution and in particular the area $A$ of the event horizon and second there is the modification of the area law itself. It is instructive to write out both terms separately:

$$
\begin{aligned}
\pi|Z|^{2} & =2 \pi \frac{\left|\widehat{q}_{0}\right|\left(\frac{1}{6} C_{A B C} p^{A} p^{B} p^{C}+\frac{1}{12} c_{2 A} p^{A}\right)}{\sqrt{\left|\widehat{q}_{0}\right|\left(\frac{1}{6} C_{A B C} p^{A} p^{B} p^{C}+\frac{1}{6} c_{2 A} p^{A}\right)}} \\
\left.4 \pi \operatorname{Im}\left(\Upsilon F_{\Upsilon}\right)\right|_{\Upsilon=-64} & =2 \pi \frac{\frac{1}{12}\left|\widehat{q}_{0}\right| c_{2 A} p^{A}}{\sqrt{\left|\widehat{q}_{0}\right|\left(\frac{1}{6} C_{A B C} p^{A} p^{B} p^{C}+\frac{1}{6} c_{2 A} p^{A}\right)}}
\end{aligned}
$$

Note that both single terms are more complicated than the full answer. The fact that they combine into one single square root depends on the precise prefactor of the term $T^{i j a b} D_{a} D^{c} T_{c b i j}$ in the highest component of the Weyl multiplet (3.62). As we saw in section 1.2.1 this is the only term besides the Einstein-Hilbert term that actually contributes to the entropy.

Moreover when expanding the area term in $c_{2 A} p^{A}$ and comparing with the corresponding expansion of the microscopic entropy formula we find a mismatch already in the leading correction term. In the expansion of the area term the leading correction term even vanishes:

$$
\frac{A}{4}=\pi|Z|^{2}=2 \pi \sqrt{\frac{1}{6}\left|\widehat{q}_{0}\right| C_{A B C} p^{A} p^{B} p^{C}}+2 \pi\left(\frac{1}{12}-\frac{1}{12}\right) c_{2 A} p^{A} \sqrt{\frac{6\left|\widehat{q}_{0}\right|}{C_{A B C} p^{A} p^{B} p^{C}}}+\cdots,
$$

whereas the expansion of (6.97) yields

$$
\mathcal{S}_{\text {micro }}=2 \pi \sqrt{\frac{1}{6}\left|\widehat{q}_{0}\right| C_{A B C} p^{A} p^{B} p^{C}}+2 \pi \cdot \frac{1}{12} \cdot c_{2 A} p^{A} \sqrt{\frac{6\left|\widehat{q}_{0}\right|}{C_{A B C} p^{A} p^{B} p^{C}}}+\cdots .
$$

The observation that the area law under very general assumptions cannot account for the microscopic entropy (6.97) was made in [125]. This then motivated the detailed investigation 91 which used Wald's entropy formula and the off-shell formalism of $\mathcal{N}=2$ supergravity to properly include the effect of higher curvature terms.

Next we would like to point out that the correction is strictly speaking not a curvature squared correction. As we saw in section 3.5.1 the curvature squared term in the highest component of the Weyl multiplet involves the square of the Weyl tensor (see formula 3.120). Since the near-horizon geometry is conformally flat this term vanishes at the event horizon and does not contribute to the entropy. The only correction term to the area law instead comes from the term $T^{i j a b} D_{a} D^{c} T_{c b i j}$ in the highest component of the Weyl multiplet which contains terms linear in the Riemann tensor as we discussed in section 4.2.1. The original proposal for a macroscopic origin of the $c_{2 A} p^{A}$ term in the entropy formula 219] was based on the observation that a term $c_{2 A} z^{A}\left(R_{a b c d}\right)^{2}$ in the Lagrangian gives the leading order term in the expansion (6.101) of the microscopic entropy Now the full supergravity analysis has shown that this

${ }^{4}$ In 219 the entropy was computed using Euclidean methods. Such methods are capable of treating deviations from the area law, and they agree with Wald's formula in all cases where both methods can be applied [66]. 
term combines with other terms and does not contribute to the entropy at all, whereas the term $T^{i j a b} D_{a} D^{c} T_{c b i j}$, which sits in the same component $\widehat{C}$ of the Weyl multiplet does not only give the leading correction but the full result.

Finally we comment on the case of black holes in $\mathcal{N}=4$ and $\mathcal{N}=8$ supergravity. Above we argued that the microscopic entropy formula (6.97) applies to these cases as well. On the supergravity side we worked with the off-shell formulation of $\mathcal{N}=2$ supergravity coupled to vector multiplets, which does not cover the full $\mathcal{N}=4,8$ theories. One can, however, describe a subsector of these theories, and for black holes which only depend on fields of this subsector it is reasonable to expect that our results apply. In particular we expect that the entropy is even in the charges and that the macroscopic entropy formula $(6.22)$ is valid. With the modified state counting of the last section the macroscopic and microscopic entropies agree in these cases as well.

Since the torus $T^{6}$ is flat the second Chern class vanishes and the entropy formula reduces to the first term. The intersection form of $T^{6}$ is $C_{A B C}=\varepsilon_{A B C}$, where $A, B, C=1,2,3$, and we get the formula

$$
\mathcal{S}_{\text {micro }}=2 \pi \sqrt{q_{0} p^{1} p^{2} p^{3}},
$$

which coincides with the Bekenstein-Hawking entropy extracted from the metric (6.87). Since higher curvature terms do not contribute, the use of the area law is justified. A microscopic explanation for this entropy formula was first given in 238, 223, 235, based on the relation between chiral null models and black hole solutions in toroidal compactifications. Note that this derivation does not use D-branes, but gives the same result, as required by U-duality.

In the case of $K 3 \times T^{2}$ the second Chern class is non-vanishing and therefore there is a subleading term in the microscopic entropy which corresponds to a higher curvature correction on the supergravity side. We refer to [224] for a more detailed account. By heterotic - type II duality the same entropy formula applies to heterotic string compactifications on $T^{6}$. The leading part of the entropy has of course the same structure (6.102) as a type II on $T^{6}$. But in addition there is a higher curvature correction.

In conclusion we see that the matching of the macroscopic and microscopic entropy depends on many subtle details. Any small mistake in one of the above points would result in a completely different and much more complicated macroscopic entropy formula. Thus the matching with the microscopic entropy is a strong argument in favour of the microscopic picture that string theory provides for black holes. Complicated as it was, our investigation was limited on the microscopic side to terms which are tree level in $\alpha^{\prime}$. A better microscopic understanding of stringy $\alpha^{\prime}$-corrections remains to be found.

\footnotetext{
${ }^{5}$ Remember that for heterotic models the coefficient of the leading term in the function $F^{(1)}$ is not related to the second Chern class of the internal manifold, but is a universal constant.
} 
122CHAPTER 6. FOUR-DIMENSIONAL $\mathcal{N}=2$ BLACK HOLES IN STRING AND M-THEORY 


\section{Chapter 7}

\section{Summary, Discussion and Outlook}

At the end of this expedition through black hole entropy in supergravity and string theory it might be useful to recall and list the key results.

1. Consider $\mathcal{N}=2$ supergravity with an arbitrary number of vector multiplets and with a general function $F(X, \widehat{A})$. Then the most general static and spherically symmetric field configuration with full $\mathcal{N}=2$ supersymmetry has the geometry $A d S^{2} \times S^{2}$ and can be fully specified in terms of the field $Z$, (4.75, 4.76).

2. By substituting this field configuration, which describes the horizon of a BPS black hole, into the generalized entropy formula (2.55) one obtains the model-independent expression (4.84) for the entropy, which specifies the entropy in terms of the field $Z$. By modelindependent we mean that the formula holds for all possible prepotentials. The formula is covariant with respect to symplectic transformations.

3. As a consequence of symplectic invariance the values of the scalar fields at the horizon are related to the charges by the stabilization equations (4.89). By solving the stabilization equations one can obtain an expression for the entropy as a function of the charges.

4. The entropy is a series in even powers of the charges and the $g$-th contribution is due to the $g$-th coupling function $F^{(g)}(X)$, see (6.5).

5. For black holes in type II compactifications the stabilization equations can be solved in the large volume limit in the three cases $p^{0}=0$ (6.21), axion-free black holes (6.27) and $\operatorname{Re} Y^{0}=0(6.30)$. We can also include a certain class of higher order terms, described by the function $G\left(Y^{0}, \Upsilon\right)$.

6. For black holes in heterotic compactifications the entropy is T-duality invariant. Neglecting higher curvature terms but taking into account all perturbative quantum corrections the entropy is related to the perturbative string coupling, evaluated at the horizon (6.51). Taking into account higher curvature corrections but working at tree level in $\alpha^{\prime}$ one can derive an explicit formula for the entropy in terms of the charges which is manifestly invariant under T-duality (6.64).

7. For black holes in $\mathcal{N}=4$ compactifications we have found the manifestly S- and T-duality invariant entropy formula (6.84). This requires to take into account non-perturbative and non-holomorphic contributions to the gravitational $C^{2}$-couplings.

8. For $\mathcal{N}=8,4,2$ compactifications on $T^{6}, K 3 \times T^{2}$ and Calabi-Yau threefolds one can derive the microscopic entropy formula (6.97) 219, 220] by counting, in the M-theory picture, the collective modes of an M5-brane wrapped on a very ample divisor. The formula agrees 
with the corresponding macroscopic entropy formula. In the cases $\mathcal{N}=2$ and $\mathcal{N}=4$ one has a subleading term which on the macroscopic side is a higher curvature correction. One has to deviate from the Bekenstein-Hawking formula and to use the generalized formula (2.5.5) in order to agree with the microscopic entropy formula.

In conclusion we have shown that the macroscopic and microscopic black hole entropies are equal for $\mathcal{N} \geq 2$ compactifications, to leading order in $\alpha^{\prime}$. The understanding of higher $\alpha^{\prime}$-corrections remains to be achieved. Moreover the state counting in the $\mathcal{N}=4$ case involves indirect arguments, based on supersymmetry or anomaly considerations. A direct derivation based on the effective M5-brane Lagrangian is desirable. Moreover we have shown that the entropy of heterotic black holes in T-duality invariant, and in the case of $\mathcal{N}=4$ compactifications, S-duality invariant, even in presence of non-trivial $C^{2}$ - and $\alpha^{\prime}$-corrections.

An intriguing feature of the entropy is its close relation to couplings in the Lagrangian. We have seen that there is a general relation between the entropy and the series $F^{(g)}(X)$ of gravitational couplings. In one example, where the higher couplings were neglected, we could prove a relation which is valid to all orders in $\alpha^{\prime}$. The relation between black hole entropy and the couplings is a natural one, once it is appreciated that the entropy in general is not just the area of the event horizon, but is given by a variation of the Lagrangian with respect to the Riemann tensor, evaluated at the horizon. Our observation also fits with the fact that the black hole attractor mechanism and the corresponding flow of the scalar fields closely resemble fixed points of the $\beta$-function and renormalization group flows. One of the important developments of the last years was the renewed interest in the relation between string theory and gauge theories. This has proceded in various steps from the discovery of the role of D-branes [11], through the matrix formulation of M-theory [239] to the AdS - CFT correspondence [52]. We think that a deeper understanding of the modified entropy formula and of the black hole attractor mechanism will help to improve our understanding of both string theory and gauge theories.

It is important to realize that most of the results discussed in this work are robust: Though we have no concrete doubts concerning specific details of string theory that we mentioned or used in the paper, we are also aware that our picture of string theory might change significantly in the next years. The discovery of string dualities is certainly a big step forward but we have become aware at the same time that we do not understand well what string theory really is. Before the second string revolution it was clear how to define string theory: it was given by the perturbative quantization of the Polyakov action in a given background geometry (and other classical background fields). Nowadays we believe in one single underlying theory, but it is not clear what are the truly fundamental objects, or more radically, whether there are truly fundamental objects at all. Therefore it is important to reflect on the assumptions that we need to make in deriving the above results. Somewhat ironically in view of the fact that we strongly advocate string theory, we find that most of the results and the most interesting results, do not depend on string theory in detail. All one has to assume is that there is a consistent quantum theory behind supergravity. At time string theory is unique as a candidate.

In the above list, the results one to five are derived on the basis of four-dimensional $\mathcal{N}=2$ supergravity, whereas the entropy counting in M-theory works by a collective mode analysis for solitons in eleven-dimensional supergravity and does not use string theory either. 17 However the other results concern the T- or S-duality invariance of entropy formulae and therefore they belong into the context of string theory and are not motivated by supergravity alone.

Let us finally indicate what are the natural concrete steps in extending the results listed above. We focussed on black hole entropy and the near horizon geometry of black holes in the presence of higher curvature terms. One should now construct the full black hole solutions. This is currently under investigation. Next one can generalize this to multi-centered solutions and to adiabatic motion in the moduli space. Such solutions can be described by superconformal quantum mechanics (see for example [240, 241, 151]), and since this is a specific case of the AdS-CFT correspondence, we can make contact with the gravity and string theory - gauge theory correspondence discussed above. We also think that a better understanding of Wald's

\footnotetext{
${ }^{1}$ This was emphasized in 219$]$.
} 
construction of surface charges is desirable and might be helpful to explore the relation between the supersymmetric attractor mechanism and renormalization group flows. Note that Wald's construction should have an interpretation in terms of cohomology, and that it resembles the descent equations that are familar from the analysis of anomalies in field theory.

Concerning black hole physics the intrinsic limitation of an approach based on BPS states is of course that one can only treat supersymmetric states. The next level of understanding will be reached when non-supersymmetric states can be brought under quantitative control. This includes in particular the Schwarzschild black hole. But as soon as one understands far non-extreme charged black holes this can be treated by switching off the charges.

Clearly the understanding of non-extreme black holes requires a major conceptual step forward. The first indication that this might be possible is provided by what one might call (in the spirit of Wigners remark concerning the relation of mathematics to physics) the unreasonable effectiveness of D-branes. Though the extrapolation to the perturbative regime is strictly speaking only justified for BPS states, it 'just works' more generally. In particular one can relate the entropy of near-extreme black holes to state counting and the resulting formula has a suggestive (though formal) interpretation in terms of branes and antibranes, see [13. Hawking radiation can be computed by considering interactions of open strings living on non-extremal D-brane configurations. Another way of going away from the extreme limit is to slightly perturb static multiblack hole solution. The dynamics of the resulting system of slowly moving, interacting black holes is determined by the metric on the moduli space of the multi-centered solution. This metric has been computed for five- and four-dimensional $\mathcal{N}=2$ supergravity coupled to vector multiplets [150, 151, 152]. In the near-coincident limit the system can be described in terms of superconformal quantum mechanics on the moduli space, see [154] for a recent review. This surprising and fascinating structure might give further insight into the entropy and dynamics of black holes. The impact of $R^{2}$-terms on the metric on moduli space is currently under investigation [153]. It seems that Wald's entropy formula appears in the metric, giving further evidence that the study of multiblack hole system will lead to a deeper understanding of black hole entropy.

Ultimately one has to understand the entropy of generic, non-extreme black holes of Schwarzschild and Kerr type. As already mentioned in the introduction there are general arguments which allow to explain the entropy of general black holes up to one order of magnitude in terms of string (or brane) states [8, 9. Moreover the Schwarzschild black hole has been analysed in the matrix formulation of M-theory [242, 243, 244. The study of stable non-BPS brane configurations (see [245] for a review) and the renewed interest in non-supersymmetric string theories [7, 246] also suggest that non-supersymmetric situations are tractable. We think that all these activities encourage the belief that the gravity / string theory - gauge theory correspondence is the right strategy for a full understanding of the entropy of non-extreme black holes.

These ideas may rise questions about the ultimate role of supersymmetry. Above we argued that our results are robust, because they can be derived to a large extent on the basis of supersymmetry, now we suggest that the we should try to do 'without supersymmetry'. But this is not necessarily a contradiction. We believe that supersymmetry plays a role at the fundamental level, and there are plenty of arguments in favour of this, which we do not need to review here. At the same time we know that our universe is in a non-supersymmetric state. In this situation it is natural to start with objects which share many of the symmetries of the vacuum, such as BPS states and then to improve on it by studying less symmetric states.

\section{Acknowledgements}

First of all I would like to thank Gabriel Lopes Cardoso and Bernard de Wit for the fruitful and pleasent collaboration over the last years. The more recent results, which are briefly summarized in section 4.4. were obtained with Jürg Käppeli as a new member of this collaboration.

I am also indepted to my other coworkers Klaus Behrndt, Gottfried Curio, Ingo Gaida, Renata Kallosh, Dieter Lüst, Swapna Mahapatra, Soo-Jong Rey and Wafic Sabra for sharing 
their insights and knowledge with me. The quantum field theory group in Halle provided a stimulating working environment for me over the last years. Many thanks for this to Robert Böhm, Holger Günther, Michael Haack, Carl Herrmann, Matthias Klein, Jan Louis and Monika Marquart. I would also like to thank Bruce Hunt for several illuminating discussions and remarks on Calabi-Yau manifolds. Part of this work was written during a stay at Cern and I would like to thank the theory division for hospitality and financial support. 


\section{Appendix A}

\section{Space-Time Geometry}

Part of the material in this appendix was taken from 92, 90, 247.

\section{A.1 Tensors}

\section{A.1.1 Metric and Vielbein}

Lorentz indices: $\mu, \nu, \ldots$ are curved indices, $a, b, \ldots$ are flat indices (local Lorentz indices, tangent space indices). They take values $0,1,2,3$.

We work with Minkowski signature $(-+++)$. The flat metric is denoted $\eta_{a b}$.

The vielbein is $e_{\mu}{ }^{a}$ and the inverse vielbein is $e_{a}{ }^{\mu}$. Thus $e_{\mu}{ }^{a} e_{a}{ }^{\nu}=\delta_{\mu}^{\nu}$. The curved and flat metrics are related by

$$
g_{\mu \nu}=e_{\mu}^{a} e_{\nu}^{b} \eta_{a b}, \quad \eta_{a b}=e_{a}^{\mu} e_{b}^{\nu} g_{\mu \nu} .
$$

Curved and flat indices are converted by

$$
V_{a}=e_{a}{ }^{\mu} V_{\mu}, \quad V_{\mu}=e_{\mu}{ }^{a} V_{a} .
$$

Curved and flat indices are moved up and down with $g_{\mu \nu}$ and $\eta_{a b}$ and their inverses $g^{\mu \nu}$ and $\eta^{a b}$, respectively. We define

$$
e=\sqrt{\left|\operatorname{det}\left(g_{\mu \nu}\right)\right|} .
$$

\section{A.1.2 Symmetrization and Antisymmetrization}

Symmetrization and antisymmetrization are done with the following normalization:

$$
V_{\left(a_{1} \cdots a_{p}\right)}=\frac{1}{p !} \sum_{\sigma} V_{\sigma a_{1} \cdots \sigma a_{p}}, \quad V_{\left[a_{1} \cdots a_{p}\right]}=\frac{1}{p !} \sum_{\sigma}(-1)^{\operatorname{sgn}(\sigma)} V_{\sigma a_{1} \cdots \sigma a_{p}},
$$

where $\sigma$ runs over all permutations of $p$ objects and $\operatorname{sgn}(\sigma)$ is the signum of $\sigma$. The normalization is such that $S_{(a b \cdots)}=S_{a b \cdots}$ for symmetric tensors $S_{a b \cdots}$ and $T_{[a b \cdots]}=T_{a b \cdots}$ for antisymmetric tensors $T_{a b \ldots}$.

The standard normalization of the completely antisymmetric four-index symbol with tangent space indices is

$$
\epsilon^{0123}=1 \text {. }
$$

When dealing with antisymmetric tensors in Minkowski signature it is convenient to use the modified symbol

$$
\varepsilon^{0123}:=i \epsilon^{0123}=i
$$

When contracting the $\varepsilon$-tensor with itself, one has to apply the Euclidean rather then the Minkowskian formula, because the signature dependent factor $(-1)^{s}$ has been absorbed by the explicit $i$ :

$$
\varepsilon_{k_{1} \cdots k_{p}, i_{1} \cdots i_{q}} \varepsilon^{k_{1} \cdots k_{p}, j_{1} \cdots j_{q}}=p ! \delta_{i_{1} \cdots i_{q}}^{j_{1} \cdots j_{q}}
$$


where

$$
\delta_{j_{1} \cdots j_{n}}^{i_{1} \cdots i_{n}}:=\operatorname{det}\left(\begin{array}{ccc}
\delta_{j_{1}}^{i_{1}} & \cdots & \delta_{j_{1}}^{i_{n}} \\
\vdots & & \vdots \\
\delta_{j_{n}}^{i_{1}} & \cdots & \delta_{j_{n}}^{i_{n}}
\end{array}\right)
$$

The fully antisymmetric tensor density (with world indices) is

$$
\varepsilon^{\mu \nu \rho \sigma}=i \epsilon^{\mu \nu \rho \sigma}=e e_{a}{ }^{\mu} e_{b}{ }^{\nu} e_{c}{ }^{\rho} e_{d}^{\sigma} \varepsilon^{a b c d} .
$$

In the main text various kinds of $\varepsilon$-symbols appear, like the $S U(2) \varepsilon$ symbol $\varepsilon_{i j}, i=1,2$ and the binormal $\varepsilon_{a b}, a, b=0,1$. We use the letter $\varepsilon$ rather then $\epsilon$ to avoid confusion with the $Q$ supersymmetry parameters $\epsilon^{i}, \epsilon_{i}$. Note however that all the other $\varepsilon$-symbols are conventionally normalized, i.e. they are real.

\section{A.1.3 Selfdual and Antiselfdual Antisymmetric Tensors}

The dual of an antisymmetric Lorentz tensor is

$$
\tilde{F}_{a b}=\frac{1}{2} \varepsilon_{a b c d} F^{c d} .
$$

The selfdual and antiselfdual parts of $F_{a b}$ are

$$
F_{a b}^{ \pm}=\frac{1}{2}\left(F_{a b} \pm \tilde{F}_{a b}\right) .
$$

Note that $F_{a b}^{ \pm}$are complex conjugated quantities in Minkowski space: $\left(F_{a b}^{+}\right)^{*}=F_{a b}^{-}$.

The projection operator onto the (anti-) selfdual part is

$$
\Pi_{a b}^{ \pm c d}=\frac{1}{2}\left(\delta_{[a}^{c} d_{b]}^{c} \pm \frac{1}{2} \varepsilon_{a b} c d\right)
$$

The following identities for (anti-) selfdual tensors are useful:

$$
\begin{aligned}
G_{[a[c}^{ \pm} H_{d] b]}^{ \pm} & = \pm \frac{1}{8} G_{e f}^{ \pm} H^{ \pm e f} \varepsilon_{a b c d}-\frac{1}{4}\left(G_{a b}^{ \pm} H_{c d}^{ \pm}+G_{c d}^{ \pm} H_{a b}^{ \pm}\right), \\
G_{a b}^{ \pm} H^{\mp c d}+G^{ \pm c d} H_{a b}^{\mp} & =4 \delta_{[a}^{[c} G_{b] e}^{ \pm} H^{\mp d] e}, \\
\frac{1}{2} \varepsilon^{a b c d} G_{[c}^{ \pm e} H_{d] e}^{ \pm} & = \pm G^{ \pm[a}{ }_{e} H^{ \pm b] e}, \\
G^{ \pm a c} H_{c}^{ \pm b}+G^{ \pm b c} H_{c}^{ \pm a} & =-\frac{1}{2} \eta^{a b} G^{ \pm c d} H_{c d}^{ \pm}, \\
G^{ \pm a c} H_{c}^{\mp b} & =G^{ \pm b c} H_{c}^{\mp a}, \quad G^{ \pm a b} H_{a b}^{\mp}=0 .
\end{aligned}
$$

\section{A.1.4 Spin Connection and Riemann Tensor}

The anholonomicity coefficients of the vielbein $e_{\mu}{ }^{a}$ are:

$$
\Omega_{\mu \nu a}:=2 \partial_{[\mu} e_{\nu] a} .
$$

The spin connection is

$$
\omega_{\mu a b}:=\frac{1}{2} e_{a}{ }^{\rho} \Omega_{\mu \rho b}-\frac{1}{2} e_{b}{ }^{\sigma} \Omega_{\mu \sigma a}-\frac{1}{2} e_{a}{ }^{\rho} e_{b}{ }^{\sigma} e_{\mu}{ }^{c} \Omega_{\rho \sigma c}
$$

and the Riemann tensor is

$$
R_{\mu \nu}^{a b}:=2 \partial_{[\mu} \omega_{\nu]}^{a b}-2 \omega_{[\mu}^{a c} \omega_{\nu]}^{d b} \eta_{c d} .
$$

By contraction one gets the Ricci tensor

$$
R_{\mu}{ }^{a}=R_{\mu \nu}{ }^{a b} e_{b}{ }^{\nu}
$$

and the Ricci scalar

$$
R=R_{\mu}{ }^{a} e_{a}{ }^{\mu} .
$$

The Weyl tensor is the trace-free part of the Riemann tensor,

$$
C_{\mu \nu}{ }^{a b}=R_{\mu \nu}^{a b}-2 \delta_{[\mu}^{[a} R_{\nu]}^{b]}+\frac{1}{3} R \delta_{[\mu}^{\left[{ }^{a}\right.} \delta_{\nu]}^{b]} .
$$




\section{A.1.5 Consequences of Rotational Invariance}

A rotationally invariant metric can be brought to the form

$$
d s^{2}=-e^{2 g} d t^{2}+e^{2 f}\left(d r^{2}+r^{2} \sin ^{2} \theta d \phi^{2}+r^{2} d \theta^{2}\right),
$$

with two arbitrary functions $f=f(t, r)$ and $g=g(t, r)$. If the metric is in addition static, then one can achieve $f=f(r)$ and $g=g(r)$.

In a spherically symmetric background curved indices take values $\mu, \nu, \ldots=t, r, \phi, \theta$ whereas flat indices take values $a, b, \ldots=0,1,2,3$. The vielbein and its inverse can be brought to the form

$$
e_{\mu}^{a}=\left(\begin{array}{cccc}
e^{g} & 0 & 0 & 0 \\
0 & e^{f} & 0 & 0 \\
0 & 0 & e^{f} r \sin \theta & 0 \\
0 & 0 & 0 & e^{f} r
\end{array}\right), \quad e_{a}^{\mu}=\left(\begin{array}{cccc}
e^{-g} & 0 & 0 & 0 \\
0 & e^{-f} & 0 & 0 \\
0 & 0 & e^{-f} r^{-1} \sin ^{-1} \theta & 0 \\
0 & 0 & 0 & e^{-f} r^{-1}
\end{array}\right)
$$

The following components of the spin connection are non-vanishing:

$$
\omega_{t}^{01}=-g^{\prime} e^{g-f}, \quad \omega_{\phi}^{12}=\left(1+r f^{\prime}\right) \sin \theta, \quad \omega_{\theta}^{13}=1+r f^{\prime}, \quad \omega_{\phi}^{23}=-\cos \theta .
$$

The corresponding curvature tensor $R_{\mu \nu}^{a b}$ has the components

$$
\begin{gathered}
R_{t r}^{01}=\left[g^{\prime \prime}+g^{\prime}\left(g^{\prime}-f^{\prime}\right)\right] e^{g-f}, \quad R_{t \phi}^{02}=g^{\prime}\left(1+r f^{\prime}\right) e^{g-f} \sin \theta, \quad R_{t \theta}^{03}=g^{\prime}\left(1+r f^{\prime}\right) e^{g-f} \\
R_{r \phi}^{12}=\left(r f^{\prime \prime}+f^{\prime}\right) \sin \theta, \quad R_{r \theta}^{13}=\left(r f^{\prime \prime}+f^{\prime}\right), \quad R_{\phi \theta}^{23}=-\sin \theta\left[1-\left(1+r f^{\prime}\right)^{2}\right] .
\end{gathered}
$$

With tangent-space indices, these curvatures read

$$
\begin{aligned}
& R_{01}^{01}=\left[g^{\prime \prime}+g^{\prime}\left(g^{\prime}-f^{\prime}\right)\right] e^{-2 f}, \\
& R_{02}^{02}=R_{03}^{03}=\left[f^{\prime} g^{\prime}+\frac{1}{r} g^{\prime}\right] e^{-2 f}, \\
& R_{12}^{12}=R_{13}^{13}=\left[f^{\prime \prime}+\frac{1}{r} f^{\prime}\right] e^{-2 f}, \\
& R_{23}^{23}=\left[f^{\prime 2}+\frac{2}{r} f^{\prime}\right] e^{-2 f} .
\end{aligned}
$$

The Ricci tensor $\left(R_{\mu \nu}^{a b} e_{b}^{\nu} e_{c}^{\mu}\right)$ and the Ricci scalar are:

$$
\begin{aligned}
R_{0}^{0} & =\left[g^{\prime \prime}+g^{\prime}\left(g^{\prime}+f^{\prime}\right)+\frac{2}{r} g^{\prime}\right] \mathrm{e}^{-2 f} \\
R_{1}^{1} & =\left[2 f^{\prime \prime}+g^{\prime \prime}+g^{\prime}\left(g^{\prime}-f^{\prime}\right)+\frac{2}{r} f^{\prime}\right] \mathrm{e}^{-2 f}, \\
R_{2}^{2} & =R_{3}^{3}=\left[f^{\prime \prime}+f^{\prime}\left(g^{\prime}+f^{\prime}\right)+\frac{1}{r}\left(3 f^{\prime}+g^{\prime}\right)\right] \mathrm{e}^{-2 f}, \\
R & =\left[2\left(2 f^{\prime \prime}+g^{\prime \prime}\right)+2\left(f^{\prime 2}+g^{\prime 2}+f^{\prime} g^{\prime}\right)+\frac{4}{r}\left(2 f^{\prime}+g^{\prime}\right)\right] e^{-2 f} .
\end{aligned}
$$

Finally the components of the Weyl tensor are:

$$
\begin{aligned}
C_{01}^{01}= & C_{23}^{23}=-2 C_{02}^{02}=-2 C_{03}^{03}=-2 C_{12}^{12}=-2 C_{13}^{13}= \\
& \frac{1}{3}\left[-f^{\prime \prime}+g^{\prime \prime}+\left(g^{\prime}-f^{\prime}\right)^{2}+\frac{1}{r}\left(f^{\prime}-g^{\prime}\right)\right] e^{-2 f} .
\end{aligned}
$$

A rotationally invariant antisymmetric tensor takes the form

$$
F_{t r}=F_{E}(t, r), \quad F_{\phi \theta}=F_{M}(t, r) \sin \theta,
$$


with all other independent components vanishing. If $F_{\mu \nu}$ is a field strength satisfying the field equations and Bianchi identities

$$
\partial_{\mu}\left(e F^{\mu \nu}\right)=0, \quad \varepsilon^{\mu \nu \rho \sigma} \partial_{\nu} F_{\rho \sigma}=0
$$

then

$$
F_{E}(t, r)=e^{g-f} \frac{q}{r^{2}}, \quad F_{M}(t, r)=p,
$$

with constants $q, p$ that are proportional to the electric and magnetic charge, respectively. Converting to flat indices one finds

$$
\begin{aligned}
& F_{01}=e^{-(f+g)} F_{E}(t, r)=e^{-2 f} \frac{q}{r^{2}} \\
& F_{23}=e^{-2 f} r^{-2} \sin ^{-1} \theta F_{M}(t, r)=e^{-2 f} \frac{p}{r^{2}} .
\end{aligned}
$$

The components of the dual tensor are:

$$
\tilde{F}_{01}=-i F_{23}, \quad \tilde{F}_{23}=i F_{01} .
$$

The components of the selfdual and antiselfdual parts are:

$$
\begin{aligned}
& F_{01}^{ \pm}=\frac{1}{2}\left(F_{01} \mp i F_{23}\right)=\mp i F_{23}^{ \pm} \\
& F_{01}^{ \pm}=\mp \frac{i}{2} e^{-2 f} r^{-2}\left(F_{M} \pm i e^{f-g} r^{2} F_{E}\right) .
\end{aligned}
$$

\section{A.2 Spinors}

\section{A.2.1 $\gamma$-Matrices}

The $\gamma$-matrices satisfy

$$
\gamma_{a} \gamma_{b}=\eta_{a b}+2 \sigma_{a b}, \quad \gamma_{5}=i \gamma_{0} \gamma_{1} \gamma_{2} \gamma_{3} .
$$

The symmetric and antisymmetric part of the product are

$$
\begin{aligned}
\gamma_{(a} \gamma_{b)} & =\frac{1}{2}\left\{\gamma_{a}, \gamma_{b}\right\}=\eta_{a b} \\
\gamma_{[a} \gamma_{b]} & =\frac{1}{2}\left[\gamma_{a}, \gamma_{b}\right]=2 \sigma_{a b}
\end{aligned}
$$

The following identities for $\gamma$-matrices are useful:

$$
\begin{aligned}
\sigma_{a b} & =-\frac{1}{2} \varepsilon_{a b c d} \sigma^{c d} \gamma_{5} \\
\sigma^{a b} \sigma_{a b} & =-3 \\
\gamma^{c} \sigma_{a b} \gamma_{c} & =0 \\
{\left[\gamma^{c}, \sigma_{a b}\right] } & =2 \delta_{[a}^{c} \gamma_{b]}, \\
{\left[\sigma_{a b}, \sigma^{c d}\right] } & =-4 \delta_{[a}^{[c} \sigma_{b]}^{d]},
\end{aligned}
$$

$$
\begin{aligned}
\gamma^{b} \gamma_{a} \gamma_{b} & =-2 \gamma_{a}, \\
\sigma^{c d} \sigma_{a b} \sigma_{c d} & =\sigma_{a b}, \\
\sigma^{b c} \gamma_{a} \sigma_{b c} & =0 \\
\left\{\gamma^{c}, \sigma_{a b}\right\} & =\varepsilon_{a b}^{c d} \gamma_{5} \gamma_{d} \\
\left\{\sigma_{a b}, \sigma^{c d}\right\} & =-\delta_{[a}^{c} \delta_{b]}^{d}+\frac{1}{2} \varepsilon_{a b}{ }^{c d} \gamma_{5} .
\end{aligned}
$$

Also note that if $T^{a b} \sigma_{a b}$ acts on a spinor of positive chirality, the tensor is projected onto its antiselfdual part:

$$
T^{a b} \sigma_{a b} \epsilon^{i}=T^{-a b} \sigma_{a b} \epsilon^{i}
$$




\section{A.2.2 Charge Conjugation, Dirac-, Majorana- and Weyl-Spinors}

The charge conjugation matrix $C$ is defined by:

$$
-\gamma_{a}^{T}=C \gamma_{a} C^{-1}, \quad \gamma_{5}^{T}=C \gamma_{5} C^{-1}, \quad C^{T}=-C .
$$

We work with four-component spinors. The Dirac conjugate is defined by

$$
\bar{\psi}=\psi^{+} \gamma_{0},
$$

whereas the Majorana conjugate is

$$
\psi^{M}=\psi^{T} C .
$$

Majorana spinors are subject to the reality constraint

$$
\psi^{M}=\bar{\psi},
$$

whereas Weyl spinors are subject to the chirality constraint

$$
\gamma_{5} \psi_{ \pm}= \pm \psi_{ \pm}
$$

Thus, the chiral projections of a spinor are

$$
\psi_{ \pm}=\frac{1}{2}\left(\mathbb{I} \pm \gamma_{5}\right) \psi
$$

In four-dimensional Minkowski space the Majorana and Weyl conditions are not compatible, i.e. they cannot be imposed simultaneously. The chiral projections of a Majorana spinor (which are not Majorana-Weyl spinors as explained in the last sentence) are not independent, as is the case for a Dirac spinor, but are related by

$$
\psi_{ \pm}=C^{-1, T} \gamma_{0}^{T} \psi_{\mp}^{*} .
$$

This follows by chiral decomposition of the Majorana constraint into equations for its left- and righthanded part. (We use a convention where $\gamma_{0}^{+}=-\gamma_{0}$ and $C^{*} C=-\mathbb{I}$.)

\section{A.2.3 Spinor Bilinears and Fierz Rearrangements}

Complex conjugation of spinor bilinears:

$$
(\bar{\psi} \phi)^{\star}=\bar{\phi} \psi, \quad\left(\bar{\psi} \gamma_{a} \phi\right)^{\star}=-\bar{\phi} \gamma_{a} \psi .
$$

Transpositions of bilinears of Majorana spinors:

$$
\bar{\psi} \phi=\bar{\phi} \psi, \quad \bar{\psi} \gamma_{a} \phi=-\bar{\phi} \gamma_{a} \psi .
$$

Fierz rearrangement formula:

$$
\phi \bar{\psi}=-\frac{1}{4}(\bar{\psi} \phi) \mathbb{I}-\frac{1}{4}\left(\bar{\psi} \gamma^{a} \phi\right) \gamma_{a}-\frac{1}{4}\left(\bar{\psi} \gamma_{5} \phi\right) \gamma_{5}+\frac{1}{4}\left(\bar{\psi} \gamma^{a} \gamma_{5} \phi\right) \gamma_{a} \gamma_{5}+\frac{1}{2}\left(\bar{\psi} \sigma^{a b} \phi\right) \sigma_{a b}
$$

These formulae are needed to work out the components of the Weyl multiplet. 


\section{Appendix B}

\section{Abelian Gauge Fields}

\section{B.1 Maxwell and Einstein-Maxwell Theory}

In this section we collect a few useful facts about abelian gauge fields, in particular various forms of the Lagrangian, equations of motion and Bianchi identities and about their solution in static curved backgrounds.

The Lagrangian for a single abelian gauge field is:

$$
\mathcal{L}=\frac{1}{2} F \wedge \star F=\frac{1}{4} e F_{\mu \nu} F^{\mu \nu} .
$$

The Maxwell equations in a curved background are, in the absence of sources,

$$
\nabla_{\mu} F^{\mu \nu}=0 \text { and } \epsilon^{\mu \nu \rho \sigma} \partial_{\nu} F_{\rho \sigma}=0
$$

where $\nabla_{\mu}$ is the Christoffel connection. The first equation is the Euler Lagrange equation obtained by varying the Lagrangian (B.1) with respect to the vector potential $A_{\mu}$, whereas the second is a Bianchi identity, i.e. the integrability condition for the existence of the vector potential

$$
F_{\mu \nu}=\partial_{\mu} A_{\nu}-\partial_{\nu} A_{\mu}=\nabla_{\mu} A_{\nu}-\nabla_{\nu} A_{\mu}
$$

The equations can be rewritten in various ways. Using the formula for the covariant derivative of an antisymmetric tensor the field equation becomes

$$
\frac{1}{\sqrt{-g}} \partial_{\mu}\left(\sqrt{-g} F^{\mu \nu}\right)=0 \text {. }
$$

The second equation is a Bianchi identity and therefore does not depend on the metric. Equivalent forms are

$$
\epsilon^{\mu \nu \rho \sigma} \nabla_{\nu} F_{\rho \sigma}=0 \text { or } \nabla_{\nu}\left(\epsilon^{\mu \nu \rho \sigma} F_{\rho \sigma}\right)=0 \text { or } \partial_{\nu}\left(\epsilon^{\mu \nu \rho \sigma} F_{\rho \sigma}\right)=0
$$

Here and in other equations we use that the Christoffel connection drops out because of the antisymmetrization. The curved space $\epsilon$-tensor is covariantly constant by the vielbein postulate $\nabla_{\mu} e_{\nu}^{a}=0$.

A more interesting way of rewriting the field equations is to go to the Hodge dual fields

$$
{ }^{\star} F_{\mu \nu}=\frac{1}{2} \epsilon_{\mu \nu \rho \sigma} F^{\rho \sigma} .
$$

In Minkowski space the Hodge- $\star$-operator on two-forms satisfies $\star^{2}=-1$ and therefore

$$
F_{\mu \nu}=-\frac{1}{2} \epsilon_{\mu \nu \rho \sigma}{ }^{\rho \sigma}
$$

In terms of the dual field the Maxwell equations take the form

$$
\epsilon^{\mu \nu \rho \sigma} \partial_{\nu}{ }^{\star} F_{\rho \sigma}=0 \text { and } \nabla_{\mu}^{\star} F^{\mu \nu}=0 .
$$


Thus the role of Euler-Lagrange equations and Bianchi identities is reversed, provided one introduces a dual gauge potential

$$
{ }^{\star} F_{\mu \nu}=\partial_{\mu} \breve{A}_{\nu}-\partial_{\nu} \breve{A}_{\mu} .
$$

Note that the original and the dual gauge field are not related by a local field redefinition. Moreover the duality is only valid in the absence of electric and magnetic sources.

The duality is manifest when writing both equations in the same form either as

$$
\nabla_{\mu} F^{\mu \nu}=0 \text { and } \nabla_{\mu}^{\star} F^{\mu \nu}=0
$$

or as

$$
\epsilon^{\mu \nu \rho \sigma} \partial_{\nu} F_{\rho \sigma}=0 \text { and } \epsilon^{\mu \nu \rho \sigma} \partial_{\nu}{ }^{\star} F_{\rho \sigma}=0 .
$$

In this formulation it is obvious that one cannot only exchange $F$ with ${ }^{\star} F$, but that one more generally has the freedom of a uniform $G l(2, \mathbb{R})$ rotation of the vector $\left(F_{\mu \nu},{ }^{\star} F_{\mu \nu}\right)$, i.e. one can take arbitrary linear combinations.

We can decompose the field strength into a selfdual and an antiselfdual part. Since $\star^{2}=-1$ in spaces with Minkowski signature selfdual and antiselfdual tensors are complex:

$$
{ }^{\star} F_{\mu \nu}^{+}=-i F_{\mu \nu}^{+}, \quad{ }^{\star} F_{\mu \nu}^{-}=i F_{\mu \nu}^{-} .
$$

The field strength can be decomposed as

$$
F_{\mu \nu}=F_{\mu \nu}^{+}+F_{\mu \nu}^{-}, \quad{ }^{\star} F_{\mu \nu}=-i F_{\mu \nu}^{+}+i F_{\mu \nu}^{-}
$$

and conversely the self and antiselfdual parts are obtained by projection:

$$
F_{\mu \nu}^{+}=\frac{1}{2}\left(F_{\mu \nu}+i^{\star} F_{\mu \nu}\right), \quad F_{\mu \nu}^{-}=\frac{1}{2}\left(F_{\mu \nu}-i^{\star} F_{\mu \nu}\right) .
$$

Note that the selfdual and antiselfdual parts are complex conjugated, $\left(F_{\mu \nu}^{+}\right)^{*}=F_{\mu \nu}^{-}$for Minkowski signature.

We also note that the other Lorentz scalar that one can form out of the field strength $F$ is a topological term:

$$
\mathcal{L}=\frac{1}{2} F \wedge F=\frac{1}{2}(F, \star F)=\frac{1}{4} e F_{\mu \nu}{ }^{\star} F^{\mu \nu}=\frac{1}{8} \epsilon^{\mu \nu \rho \sigma} F_{\mu \nu} F_{\rho \sigma}=\frac{1}{4} \partial_{\mu} \epsilon^{\mu \nu \rho \sigma} A_{\nu} F_{\rho \sigma} .
$$

Nevertheless such terms can play a role for example for field configurations with a non-trivial behaviour at infinity. In effective $U(1)$ field theories such terms are generated with a field depedent coefficient, and then they are relevant for the local dynamics as well.

In the main part of the paper we use a non-standard definition for the $\epsilon$-tensor with the effect that Minkowski-signature formulae look like Euclidean signature formulae. Then we use a modified definition for the dual tensor, denoted by

$$
\tilde{F}_{\mu \nu}=i^{\star} F_{\mu \nu} .
$$

Consider now finding static solutions, $\partial_{t} F_{\mu \nu}=0$, of the Maxwell equations in a 'conformastatic' curved background. Such a metric can be brought to the isotropic form

$$
d s^{2}=-e^{2 g(\vec{x})} d t^{2}+e^{2 f(\vec{x})} d \vec{x}^{2} .
$$

The corresponding world indices are denoted by $\mu=t, m=x, y, z$.

First look for electric solutions, $F_{m n}=0$. Then the Bianchi identity reduces to

$$
\partial_{m} F_{t n}=\partial_{n} F_{t m}
$$

which is solved by introducing an electrostatic potential,

$$
F_{t m}=\partial_{m} \frac{1}{H} .
$$


The field equation then implies

$$
\sum_{m=x, y, z} \partial_{m}\left(e^{2 f} \frac{1}{H^{2}} \partial_{m} H\right)=0,
$$

which is solved by

$$
e^{f}=H \text { and } \sum_{m=x, y, z} \partial_{m} \partial_{m} H=\Delta H=0,
$$

where $\Delta$ is the (flat) Laplace operator. The Einstein equation then implies $g=-f$.

Consider now a magnetic solution, $F_{t m}=0$. This time the Bianchi identities read

$$
\epsilon^{t m n p} \partial_{m} F_{n p}=0
$$

and are solved by

$$
F_{n p}=\sum_{q=x, y, z} \epsilon_{n p q} \partial_{q} H
$$

where $\epsilon_{n p q}$ is the flat $\epsilon$-symbol, $\epsilon_{x y z}=1$ and $H$ must be a harmonic function,

$$
\sum_{q=x, y, z} \partial_{q} \partial_{q} H=0
$$

Then the field equation is satisfied if one takes $f=-g$ and finally the Einstein equation implies $e^{f}=H$.

Both solutions are related by a electric-magnetic duality rotation and belong to a class of solutions of Einstein-Maxwell theory called the Majumdar Papapetrou solutions. The general form of the solution is

$$
\begin{gathered}
d s^{2}=-H^{-2} d t^{2}+H^{2} d \vec{x}^{2}, \\
F_{\mu \nu}=\cos \theta F_{\mu \nu}^{(0)}+\sin \theta^{\star} F_{\mu \nu}^{(0)}, \\
F^{(0)}:=\frac{1}{2} F_{\mu \nu}^{(0)} d x^{\mu} \wedge d x^{\nu}=\vec{\nabla} \frac{1}{H} d \vec{x} \wedge d t,
\end{gathered}
$$

where $H$ is a harmonic function,

$$
\Delta H=\sum_{m=x, y, z} \partial_{m} \partial_{m} H=0 .
$$

The parameter $\theta$ parametrizes an electric-magnetic duality rotation.

The most simple solution, which is given by a spherically symmetric choice

$$
H=1+\frac{M}{r}
$$

is the dyonic extreme Reissner-Nordstrom black hole with mass $\mathrm{M}$ and electric and magnetic charges $p, q$ given by $q=\cos \theta M$ and $p=\sin \theta M$. Thus the mass is related to the charges by $M^{2}=p^{2}+q^{2}$, and the values $\theta=0, \frac{\pi}{2}, \pi, \frac{3 \pi}{2}$ of the parameter $\theta$ correspond to the cases of positive electric, postive magnetic, negative electric and negative magnetic charge, respectively. Our duality rotation is an $S O(2)$ transformation rather then a general $G l(2, \mathbb{R})$ transformation in order to keep the normalization of the electric and magnetic charge fixed.

A generalization of this is provided by the multi-centered solution, where

$$
H=1+\sum_{i=1}^{N} \frac{M_{i}}{\left|\vec{x}-\overrightarrow{x_{i}}\right|} .
$$

The $\vec{x}_{i}$ are arbitrary points in space. When approaching any of these points the metric becomes asymptotic to the Bertotti-Robinson metric. The 'points' $\overrightarrow{x_{i}}$ represent the postions of the finite size horizons of Reissner-Nordstrom black holes in (asymptotically) isotropic coordinates. The 
electric and magnetic charges and the mass of the $i$-th black hole are given by $M_{i}^{2}=p_{i}^{2}+q_{i}^{2}$. Note that this configuration has the astonishing property that it is static, i.e. the gravitational attraction and the electro- and magneto-static repulsion cancel for all choices of the locations $\vec{x}_{i}$ of the black holes. In particular the mass is additive and is given by the total charge. Note that to achieve a static solution all the black holes must have charges with the same 'sign' in the sense that all the complex charges $q_{i}+i p_{i}$ must lay on the same ray in the complex plane, i.e. one does not have the freedom to make electric-magnetic duality rotations on individual black holes. In order to avoid naked singularities, all the parameters $M_{i}$ in (B.30) have to be positive. The field equations fix the relation between $M_{i}$ and $q_{i}+i p_{i}$ up to a uniform duality rotation [248].

One can consider more general choices for the harmonic functions, but it was shown in 74 that all other choices lead to naked singularities.

\section{B.2 Abelian Gauge Fields coupled to $\mathcal{N}=2$ Supergravity}

We now generalize to the case of $N$ abelian gauge fields with Lagrangian

$$
\mathcal{L}=e \frac{1}{2}\left(-\gamma_{I J} F_{\mu \nu}^{I} F^{J \mu \nu}+\theta_{I J} F_{\mu \nu}^{I{ }^{\star}} F^{J \mu \nu}\right) .
$$

$\gamma_{I J}$ generalizes the gauge coupling $1 / g^{2}$ that we surpressed above. The gauge kinetic term of abelian vector multiplets coupled to $\mathcal{N}=2$ supergravity takes precisely this form with couplings $\gamma_{I J}$ and 'theta-angles' $\theta_{I J}$ that are field-dependent.

Now introduce a complex symmetric coupling matrix:

$$
\mathcal{N}_{I J}=-i \gamma_{I J}+\theta_{I J}
$$

and rewrite the Lagrangian:

$$
\begin{aligned}
\mathcal{L} / e= & \frac{1}{2}\left(-F^{I} \gamma_{I J} F^{J}+F^{I} \theta_{I J^{\star}} F^{J}\right)=\frac{i}{2}\left(F^{+I} \mathcal{N}_{I J} F^{+J}-\text { c.c. }\right) \\
& =-\operatorname{Im}\left(F^{+I} \mathcal{N}_{I J} F^{+J}\right)=\operatorname{Im}\left(F^{-I} \overline{\mathcal{N}}_{I J} F^{-J}\right) .
\end{aligned}
$$

The Euler-Lagrange equations for the vector fields are

$$
\nabla_{\mu}\left(\mathcal{N}_{I J} F^{+J \mu \nu}-\text { c.c. }\right)=0
$$

and the Bianchi identities

$$
\epsilon^{\mu \nu \rho \sigma} \partial_{\nu} F_{\rho \sigma}^{I}=0
$$

can be rewritten as

$$
\nabla_{\mu}\left(F^{+I \mu \nu}-F^{-I \mu \nu}\right)=0 .
$$

As is the case in Maxwell theory one can cast the Euler-Lagrange equations and Bianchi identities into a symmetric form, which displays a continuous electric-magnetic duality symmetry (in the absence of sources). To do so in this more general situation one defines a set of so-called dual gauge fields by

$$
G_{I \mu \nu}^{+}=\mathcal{N}_{I J} F_{\mu \nu}^{+J}
$$

Then the Euler-Lagrange equations read

$$
\nabla_{\mu}\left(G_{I}^{+\mu \nu}-G_{I}^{-\mu \nu}\right)=0
$$

When considering the full $\mathcal{N}=2$ Lagrangian the situation is slightly more general because additional moment couplings $O_{I \mu \nu}^{+} F^{+I \mu \nu}+$ c.c. are present. These describe fermion couplings, and in the off-shell formulation the coupling to a bosonic auxiliary field. Then, the dual gauge fields are defined by

$$
\mathcal{L} / e=\frac{i}{2}\left(F_{\mu \nu}^{+I} G_{I}^{+\mu \nu}-\text { h.c. }\right)
$$


or equivalently by

$$
G_{I}^{+\mu \nu}=e^{-1} \frac{2}{i} \frac{\partial \mathcal{L}}{\partial F_{\mu \nu}^{I+}} .
$$

With this definition the Euler Lagrange equations and Bianchi identities still have the form given above.

The combined system of Euler-Lagrange equations and Bianchi identities is manifestly invariant under duality rotations:

$$
\left(\begin{array}{c}
F^{ \pm I} \\
G_{J}^{ \pm}
\end{array}\right) \longrightarrow\left(\begin{array}{cc}
U_{K}^{I} & Z^{I L} \\
W_{J K} & V_{J}{ }^{L}
\end{array}\right)\left(\begin{array}{c}
F^{ \pm K} \\
G_{L}^{ \pm}
\end{array}\right)=\left(\begin{array}{c}
\breve{F}^{ \pm I} \\
\breve{G}_{J}^{ \pm}
\end{array}\right) .
$$

A detailed discussion of duality in this case was given in the main text.

Due to the presence of field dependent couplings the field equations are now harder to solve. In a static background one can, however, still express the magnetic parts of the field strength and its dual in terms of harmonic functions. This is due to the fact that the equations take the form of Bianchi identities, which do not involve the metric:

$$
\begin{aligned}
\epsilon^{\mu \nu \rho \sigma} \partial_{\nu} F_{\rho \sigma}^{I} & =0, \\
\epsilon^{\mu \nu \rho \sigma} \partial_{\nu} G_{J \rho \sigma} & =0 .
\end{aligned}
$$

Looking for static solutions in a static metric background (B.17) this is solved by

$$
\begin{aligned}
F_{m n}^{I} & =\sum_{p} \epsilon_{m n p} H^{I}, \\
G_{J m n} & =\sum_{p} \epsilon_{m n p} H_{J},
\end{aligned}
$$

where $H^{I}, H_{J}$ are harmonic functions,

$$
\Delta H^{I}=0=\Delta H_{J} .
$$

Since the electric parts $F_{t m}^{I}, G_{J t m}$ are determined by the magnetic parts, this already fixes the gauge fields. But in order to explicitly specify the electric parts one needs to know the functions $f, g$ and the metric and the field dependent matrix $\mathcal{N}_{I J}$. This requires to solve the gravitational and scalar field equations.

This has been done explicitly for various cases (but typically in cases where half of the electric and magnetic charges have been set to zero). The resulting metrics generalize the MajumdarPapapetrou solutions and describe static configurations of extreme black holes carrying electric and magnetic charges with respect to several gauge fields. Generically theses solutions also contain nontrivial space-dependent scalar fields. The functions $f$ and $g$ are still related by $f=-g$ as a consequence of supersymmetry. They are complicated functions of the harmonic functions $H^{I}, H_{J}$ that parametrize the solution.

In the main text we need the explicit solution for a metric which is spherically symmetric in addition to static. Introducing spheric coordinates in the space part the metric takes the form

$$
d s^{2}=-e^{2 g} d t^{2}+e^{2 f}\left(d r^{2}+r^{2}\left(\sin \theta d \phi^{2}+d \theta^{2}\right)\right) .
$$

We denote world indices by $t, r, \phi, \theta$. Then we can take the magnetic gauge fields to have the form

$$
F_{\phi \theta}^{I}(r)=-\partial_{r} H^{I}(r), \quad G_{J \phi \theta}(r)=-\partial_{r} H_{J}(r) .
$$

Spherical symmetry requires to take the harmonic functions to be of single-centered type,

$$
H^{I}=h^{I}+\frac{p^{I}}{r}, \quad H_{J}=h_{J}+\frac{q_{J}}{r},
$$


with the result

$$
F_{\phi \theta}^{I}=\frac{p^{I}}{r^{2}}, \quad G_{J \phi \theta}=\frac{q_{J}}{r^{2}} .
$$

Switching to flat indices we find

$$
F_{23}^{I}=\frac{e^{-2 f}}{r^{2}} p^{I}, \quad G_{J 23}=\frac{e^{-2 f}}{r^{2}} q_{J},
$$

which is what we use in the main text. For a single gauge field with no coupling to scalar fields we have $G_{23}=F_{01}$ and we have rederived the result we found in appendix $A$. 


\section{Appendix $\mathrm{C}$}

\section{Covariant Derivatives}

We review the definition of covariant quantities and covariant derivatives given in 93, 92]: a quantity is covariant with respect to a local transformation if the transformation law does not involve derivatives of the transformation parameter. A derivative is called covariant if its application to a covariant quantity gives another covariant quantity. A given derivative can be made covariant by adding an object that transforms appropriately (namely the connection related to the transformation under consideration). This process we call covariantization, and we illustrate it in a schematic example.

Let $\phi$ be a field that transforms as

$$
\delta \phi(x)=\epsilon(x) \psi(x),
$$

where $\epsilon(x)$ is the transformation parameter. (In general the fields $\phi, \psi$ and the parameter $\epsilon$ can be tensors or spinors with respect to several transformation groups. Note also that $\psi$ could be identical to $\phi$ as is the case for abelian gauge transformations or dilatations for instance. The relevant fact we want to focus on in our example is that the transformation is linear in a space-time dependent parameter.) Now consider how the derivative transforms:

$$
\delta \partial_{\mu} \phi=\epsilon \partial \psi+\partial \epsilon \psi .
$$

This is not covariant according to our definition because of the second term. To covariantize we add a second term to the derivative

$$
D_{\mu} \phi=\partial_{\mu} \phi-h_{\mu} \psi,
$$

where the newly introduced connection $h_{\mu}$ has to transform as

$$
\delta h_{\mu} \psi=\partial_{\mu} \epsilon \psi+\cdots .
$$

As indicated there might be further terms which do not contain $\partial_{\mu} \epsilon$ and are linear in $\epsilon$. Such additional terms are in fact present in many cases, in particular in $\mathcal{N}=2$ supertransformations. Note that the second term contains the transformed field $\psi$ rather than the original field $\phi$. Thus, as an operator, the covariant derivative has the form

$$
D_{\mu}=\partial_{\mu}-\delta\left(h_{\mu}\right),
$$

where the operator $\delta\left(h_{\mu}\right)$ generates a transformation with parameter $h_{\mu}$ on the object to its right (compare to (C.1.C.3)). The new covariant derivative transforms covariantly:

$$
\delta D_{\mu} \phi=\epsilon\left(\partial_{\mu} \psi+\cdots\right) .
$$

When considering several kinds of transformations, one has to add several connections,

$$
D_{\mu}=\partial_{\mu}-\sum_{T} \delta\left(h_{\mu}(T)\right) .
$$


In the superconformal context $D_{\mu}$ denotes the covariant derivative with respect to all superconformal transformations, whereas $\mathcal{D}_{\mu}$ is the convariant derivative with respect to Lorentz transformations, dilatations, $S U(2)_{R} \times U(1)_{R}$ and gauge transformations. $\mathcal{D}_{\mu}$ is useful when going to the Poincaré gauge.

As a concrete example let us work out the covariant derivative of $X^{I}$, which has Weyl and chiral weights $w=1, c=-1$. First covariantize with respect to $U(1)$ transformations and denote the corresponding covariant derivative by $\mathcal{D}_{\mu}^{\prime}$ :

$$
\delta X^{I}=-i \alpha X^{I}, \quad \mathcal{D}_{\mu}^{\prime} X^{I}=\partial_{\mu} X^{I}-h_{\mu}(A) X^{I}, \quad \delta h_{\mu}=-i \partial_{\mu} \alpha .
$$

Since $h_{\mu}(A)=-i A_{\mu}$ this means $\delta A_{\mu}=\partial_{\mu} \alpha$.

After covariantization with respect to dilatations we get the full $\mathcal{D}_{\mu}$ since $X^{I}$ is neutral with respect to the other relevant transformations:

$$
\mathcal{D}_{\mu} X^{I}=\left(\partial_{\mu}-b_{\mu}+i A_{\mu}\right) X^{I}=\partial_{\mu} X^{I}-w b_{\mu} X^{I}-i c A_{\mu} X^{I} .
$$

To obtain the superconformal derivative $D_{\mu}$ we have to take into account that $X^{I}$ transform under $Q$-supertransformations:

$$
\delta X^{I}=\bar{\epsilon}^{i} \Omega_{i}^{I} .
$$

Therefore further covariantization is needed:

$$
D_{\mu} X^{I}=\mathcal{D}_{\mu} X^{I}-\frac{1}{2} \bar{\psi}_{\mu}^{i} \Omega_{i}^{I},
$$

where we used $h_{\mu}^{i}(Q)=\frac{1}{2} \psi_{\mu}^{i}$ and that $\psi_{\mu}^{i}$ transforms into $\mathcal{D}_{\mu} \bar{\epsilon}^{i}+\cdots$. 


\section{Appendix D}

\section{Modular Geometry}

In this appendix we illustrate the geometry of vector multiplet moduli spaces using the most simple examples.

The vector multiplet moduli space of a four-dimensional $\mathcal{N}=2$ supergravity theory or string compactification is a special Kähler manifold. In the most simple case this is a symmetric space. This happens for example in tree level heterotic compactifications, where the moduli space is locally

$$
\frac{S U(1,1)}{U(1)} \times \frac{S O\left(2, N_{V}-1\right)}{S O(2) \times S O\left(N_{V}-1\right)} .
$$

In the following we will discuss some local and global properties of the moduli space. The fact that it is special Kähler will not play an explicit role. Using the local isomorphisms

$$
\frac{S U(1,1)}{U(1)} \simeq \frac{S O(2,1)}{S O(2)} \simeq \frac{S L(2, \mathbb{R})}{S O(2)} \text { and } \frac{S O(2,2)}{S O(2) \times S O(2)} \simeq \frac{S O(2,1)}{S O(2)} \times \frac{S O(1,2)}{S O(2)}
$$

we realize that for $N_{V} \leq 3$ the moduli space locally is a product of $S L(2, \mathbb{R}) / S O(2)$ cosets. This is the space we will consider in the following. The standard realization is given by the complex upper half plane

$$
\mathcal{H}=\{\tau \in \mathbb{C} \mid \operatorname{Im} \tau>0\}
$$

equipped with the Poincaré metric

$$
d s^{2}=(\operatorname{Im} \tau)^{-2} d \tau d \bar{\tau}
$$

which is a Kähler metric with Kähler potential

$$
K=-\log (i(\tau-\bar{\tau})) .
$$

The group $S L(2, \mathbb{R})$ acts from the left on the coset by fractional linear transformations,

$$
\tau \rightarrow \frac{a \tau+b}{c \tau+d}, \text { where }\left(\begin{array}{ll}
a & b \\
c & d
\end{array}\right) \in S L(2, \mathbb{R}) .
$$

Since the matrix $-\mathbb{I}$ acts trivially, the group which properly acts on $\mathcal{H}$ is the projective group $\operatorname{PSL}(2, \mathbb{R})$.

A trivial reparametrization is obtained by replacing the upper half plane $\mathcal{H}$ by the right half plane $\{t \in \mathbb{C} \mid t=-i \tau, \tau \in \mathcal{H}\}$. This is the standard parametrization for heterotic moduli, whereas for IIA moduli one conventionally prefers the upper half plane. A less trivial reparametrization is provided by a conformal transformation that maps the upper halfplane onto the interior of the unit disc. This is the natural realization of $S U(1,1) / U(1)$. Realizations of non-compact symmetric spaces by bounded open domains are somewhat distinguished. In particular they are rare. The spaces (D.1) have several realizations by unbounded but only one 
realization by a bounded domain. Explicit parametrizations of the higher dimensional members of the family (D.1) can be found in [33, 195].

In string theory the global structure of the moduli space is determined by the discrete duality group. For moduli spaces of local structure $G / H$ (where $G$ is non-compact, $H$ is a maximal compact subgroup and $G / H$ is symmetric, as is the case for supergravity models with sufficiently many supercharges, and toroidal string compactifications) the discrete group is a discrete subgroup $G(\mathbb{Z})$ of $G$. The true moduli space is then the left-right coset $G(\mathbb{Z}) \backslash G / H$, whereas the covering space $G / H$ is sometimes called the Teichmüller space ( borrowing terminology from Riemann surfaces). Depending on the context $G(\mathbb{Z})$ is called the T, S or U-duality group. The relevant case for our purposes is $G=S L(2, \mathbb{R}), H=S O(2)$ and $G(\mathbb{Z})=S L(2, \mathbb{Z})$. Depending on the actual case $S L(2, \mathbb{Z})$ is either T-duality or S-duality.

The presence of the discrete duality group implies that points related by the action (D.6), with integer values of $a, b, c, d$ are to be identified. Since $S L(2, \mathbb{Z})$ is generated by the elements $\tau \rightarrow \tau+1$ and $\tau \rightarrow \frac{-1}{\tau}$, a realization of the moduli space is provided by the fundamental domain

$$
\mathcal{F}=\left\{\tau \in \mathcal{H} \mid-\frac{1}{2}<\operatorname{Re} \tau<\frac{1}{2} \text { and }|\tau|>1\right\}
$$

when supplemented by adding boundary points such that all inequivalent points occur once. The boundary contains the fixed points $\infty, i, \rho=\exp (2 \pi i / 3)$ of the modular group, which are relevant for the discussion of gauge symmetry enhancement.

Since physical quantities have to be duality invariant, automorphic forms and automorphic functions of the group $G(\mathbb{Z})$ make their appearence. We discuss such objects for the most simple case $S L(2, \mathbb{Z})$. Part of the following material is taken from 249].

A function has weight $k$ under $S L(2, \mathbb{Z})$, iff

$$
f(\gamma \cdot z)=(c z+d)^{k} f(z)
$$

for $\gamma \in S L(2, \mathbb{Z})$. Holomorphic weight $k$ functions on $H$ are classified according to their behaviour at $\infty$ : They are called modular functions, modular forms, or cusp forms, iff they are meromorphic, holomorphic or vanishing at $\infty$, respectively.

We now turn to explicit examples. For $k \geq 4$ and $k$ even

$$
G_{k}(z)=\sum_{(m, n) \neq(0,0)} \frac{1}{(m z+n)^{k}}
$$

is the $k$-th Eisenstein series. It is absolutely convergent and defines a modular form of degree $k$. $G_{k}$ is finite at $\infty$ :

$$
G_{k}(\infty)=\sum_{n \neq 0} n^{-k}=2 \zeta(k) .
$$

The values of the $\zeta$ function at even $k$ are related to the Bernoulli numbers:

$$
\zeta(k)=-\frac{(2 \pi i)^{k}}{2} \frac{B_{k}}{k !},
$$

where the Bernoulli numbers are defined by

$$
\frac{x}{e^{x}-1}=\sum_{k=0}^{\infty} B_{k} \frac{x^{k}}{k !} .
$$

The normalized Eisenstein series are

$$
E_{k}(z)=\frac{1}{2 \zeta(k)} G_{k}(z)=1-\frac{2 k}{B_{k}} \sum_{n=1}^{\infty} \sigma_{k-1}(n) q^{n} .
$$

They have rational coefficients in the $q=e^{2 \pi i z}$ expansion. The coefficents of the $q$ expansion are arithmetic:

$$
\sigma_{k-1}(n)=\sum_{d \mid n} d^{k-1},
$$


where the sum is over all divisors of $n$.

The second Eisenstein series is only conditionally convergent. Choosing

$$
E_{2}(z)=1+\frac{6}{\pi^{2}} \sum_{m=1}^{\infty} \sum_{n=-\infty, \neq 0}^{\infty} \frac{1}{(m z+n)^{2}}=1-24 \sum_{n=1}^{\infty} \sigma_{1}(n) q^{n}
$$

gives a holomorphic function which has anomalous, inhomogenous behaviour under modular transformations:

$$
z^{-2} E_{2}\left(-\frac{1}{z}\right)=E_{2}(z)+\frac{12}{2 \pi i z} .
$$

The non-holomorphic but modular covariant (weight 2) Eisenstein series is defined by

$$
\widehat{G}_{2}(\tau, \bar{\tau})=G_{2}(\tau)-\frac{2 \pi i}{\tau-\bar{\tau}} .
$$

The absolute modular invariant $j$ function

$$
j(z)=1728 \frac{E_{4}^{3}(z)}{E_{4}^{3}(z)-E_{6}^{2}(z)}
$$

is a modular function of weight 0 , and all modular functions of weight 0 are rational functions of $j$. $j$ has a simple pole with residue 1 at $\infty$ and is holomorphic in $H$. Some special values are $j(\rho)=0, j^{\prime}(\rho)=0, j^{\prime \prime}(\rho)=0, j^{\prime \prime \prime}(\rho) \neq 0$, where $\rho=e^{2 \pi i / 3}$ and $j(i)=1728, j^{\prime}(i)=0, j^{\prime \prime}(i) \neq 0$. The order of these zeros plays a crucial role in the discussion of gauge symmetry enhancement.

The $\eta$ function

$$
\eta(z)=e^{2 \pi i z / 24} \prod_{n=1}^{\infty}\left(1-e^{2 \pi i n z}\right)
$$

is a modular form of weight $1 / 2$ up to phase:

$$
\eta\left(-\frac{1}{z}\right)=\sqrt{\frac{z}{i}} \eta(z),
$$

where the root has nonnegative real part.

The $\eta$ function is related to $E_{2}(z)$ by:

$$
\frac{\eta^{\prime}(z)}{\eta(z)}=\frac{2 \pi i}{24} E_{2}(z)
$$

The derivative of a modular form is in general not a modular form. A covariant derivative can be defined, using the inhomogenous transformation property of $E_{2}$ to cancel the 'anomaly':

$$
D f(z)=f^{\prime}(z)-2 \pi i \frac{k}{12} E_{2}(z) f(z) .
$$

This covariant derivative relates modular forms to modular forms.

Any modular form of weight $k$ is a polynomial in $E_{4}$ and $E_{6}$ :

$$
f(z)=\sum_{4 i+6 j=k} c_{i j} E_{4}^{i}(z) E_{6}^{j}(z) .
$$

A modular form of weight $k$ vanishes at $i$, iff $k \neq 0$ modulo 4 and vanishes at $\rho$, iff $k \neq 0$ modulo 3 .

A modular form of weight $k$ with multiplyer system is by definition an object that transforms as

$$
f(\gamma \cdot z)=e^{i \Phi(\gamma)}(c z+d)^{k} f(z) .
$$


$\eta$ is a form of weight $1 / 2$ with non-trivial multiplyer system. Any such generalized modular form with weight $k$ which is regular in the fundamental domain can be written as

$$
f(z)=\eta^{2 k}\left(\frac{G_{6}}{\eta^{12}}\right)^{m}\left(\frac{G_{4}}{\eta^{8}}\right)^{n} P(j)
$$

where $m, n \in \mathbb{N}$ and $P$ is a polynomial.

Note that one can also consider modular forms of negative weight which then have poles at $i$ and $\rho$ instead of zeros.

The theory of automorphic or modular forms of more complicated groups is less developed. There seems to be a deep relation to infinite dimensional Lie algebras such as Borcherds algebras 195. Both structures are intimately related in string theory.

Since we mentioned the T-duality group $O\left(2, N_{V}-1, \mathbb{Z}\right)$ several times in the paper, we illustrate the complications involved in properly defining and describing this group using the example $O(2,2, \mathbb{Z})$ which is the T-duality group of a two-torus 1 Whereas the corresponding continuous group locally factorizes as $O(2,2) \simeq S L(2, \mathbb{R}) \times S L(2, \mathbb{R})$ it is by no means true that $O(2,2, \mathbb{Z})$ is just the direct product of two $S L(2, \mathbb{Z})$ groups, where one acts on the complex structure modulus whereas the other acts on the (complexified) Kähler modulus. The full Tduality group contains two more $\mathbb{Z}_{2}$ transformations. One of them is mirror symmetry, which exchanges the Kähler and the complex structure modulus and, hence, the two $S L(2, \mathbb{Z})$ groups. The second (which we ignored in the main part) is world sheet parity. A detailed description of this group and its fundamental domain is found in [250, 187, 158.

\footnotetext{
${ }^{1}$ The structure of the group $O(d, d, \mathbb{Z})$ is discussed in 187 .
} 


\section{Appendix E}

\section{The Polylogarithmic Functions}

We quote the definition and some basic properties of the polylogarithmic functions, see for example [195, 231, 207].

The series

$$
L i_{k}(x)=\sum_{n=1}^{\infty} \frac{x^{k}}{n^{k}}, \text { for } \quad 0<x<1
$$

can be extended to a multivalued analytic function on $\mathbb{C}$, called the $k$-th polylogarithmic function, or $k$-th polylog for short.

In particular we have $L i_{1}(x)=-\log (1-x)$ and $x \frac{d}{d x} L i_{k}(x)=L i_{k-1}(x)$. Special values are $L i_{k}(x \rightarrow 0+)=0$ and $L i_{k}(1)=\zeta(k)$. Some integral representations:

$$
\begin{aligned}
L i_{2}(x) & =\int_{0}^{1} \frac{d t}{t} \frac{1}{1-x t} \\
L i_{3}(x) & =-\int_{0}^{1} \frac{d t}{t} \int_{0}^{1} \frac{d s}{s} \log (1-x t s)
\end{aligned}
$$

In non-perturbative world-sheet or space-time contributions in string theory one encounters expressions of the type $L i_{k}\left(e^{-z}\right)$, where $z \rightarrow \infty$ corresponds to the classical or large volume limit and $z \rightarrow 0$ corresponds to a special locus, for example the boundary of the Kähler cone or the boundary of a Weyl chamber. In the classical limit this expression goes exponentially to zero, which shows that the polylogarithmic term is non-perturbative either in $\alpha^{\prime}$ or in $g_{S}$, since $z$ is linear in the moduli. Concerning the behaviour on special loci we note that

$$
\begin{aligned}
& L i_{3}\left(e^{-z}\right)=\zeta(3)-\frac{\pi^{2}}{6} z+\left(\frac{3}{4}-\frac{\log (z)}{2}\right) z^{2}+\mathcal{O}\left(z^{3}\right), \\
& L i_{2}\left(e^{-z}\right)=\frac{\pi^{2}}{6}+(\log (z)-1) z+\frac{z^{2}}{2}+\mathcal{O}\left(z^{3}\right)
\end{aligned}
$$

where we used $\zeta(2)=\frac{\pi^{2}}{6}$.

For $k \leq 0$ the polylog is an elementary function:

$$
\begin{gathered}
L i_{0}(x)=\frac{x}{1-x}, \\
L i_{k}(x)=\left(x \frac{d}{d x}\right)^{|k|} \frac{1}{1-x}, \quad k<0 .
\end{gathered}
$$

These functions appear in the higher gravitational couplings $\mathcal{F}^{(g>1)}$. 


\section{Bibliography}

[1] V.P. Frolov and I.D. Novikov, Black Hole Physics (Kluwer Academic Publishers, 1998).

[2] Geo 600 home page, http://www.geo600.uni-hannover.de.

[3] Virgo project central web site, http://www.virgo.infn.it.

[4] Ligo homepage, http://www.ligo.caltech.edu.

[5] M.B. Green, J.H. Schwarz, and E. Witten, Superstring Theory (Cambridge University Press, 1987).

[6] D. Lüst and S. Theisen, Lectures on String Theory, Lecture Notes in Physics Vol. 346 (Springer-Verlag, 1989).

[7] J. Polchinski, String Theory (Cambridge University Press, 1998).

[8] L. Susskind, Some Speculations about Black Hole Entropy in String Theory, in The Black Hole, edited by C. Teitelboim, pp. 118 - 131, 1993, hep-th/9309145.

[9] G.T. Horowitz and J. Polchinski, Phys. Rev. D55, 6189 (1997), hep-th/9612146.

[10] G.W. Gibbons, Supersymmetric Soliton States in Extended Supergravity Theories, in Unified Theories of Elementary Particles, edited by P. Breitenlohner and H. Dürr, Springer, 1982, Lecture Notes in Physics 160.

[11] J. Polchinski, Phys. Rev. Lett. 75, 4724 (1995), hep-th/9510017.

[12] A. Strominger and C. Vafa, Phys. Lett. B379, 99 (1996).

[13] J. Maldacena, Black Holes in String Theory, PhD thesis, Princeton U., 1996, hep-th/9607235.

[14] T. Mohaupt, Black Holes in Supergravity and String Theory, Lecture Notes, submitted to Class. Quant. Grav., hep-th/0004098.

[15] B. de Wit, J.W. van Holten and A. van Proeyen, Nucl. Phys. B184, 77 (1981), (E.: ibid. B222, $516(1983))$.

[16] B. de Wit, P.G. Lauwers and A. van Proeyen, Nucl. Phys. B255, 569 (1985).

[17] E. Cremmer, C. Kounnas, A. Van Proeyen, J.P. Derendinger, S. Ferrara, B. de Wit and L. Girardello, Nucl. Phys. B250, 385 (1985).

[18] R.M. Wald, Phys. Rev. D48, 3427 (1993), gr-qc/9307038.

[19] G.L. Cardoso, B. de Wit, J. Käppeli and T. Mohaupt, Stationary BPS Solutions in $N=2$ Supergravity with $R^{2}$ Interactions, hep-th/0009234, 2000.

[20] C.W. Misner, K.S. Thorne and J.A. Wheeler, Gravitation (Freeman, 1973). 
[21] R.M. Wald, General Relativity (Universty of Chicago Press, 1984).

[22] S.W. Hawking and G.F.R. Ellis, The large scale structure of space-time (Cambridge University Press, 1973).

[23] A.G. Riess et al., Astronomical Journal 116, 1009 (1998).

[24] S. Weinberg, Rev. Mod. Phys. 61, 1 (1989).

[25] G. 't Hooft, Nucl. Phys. B62, 444 (1973).

[26] G. 't Hooft and M. Veltman, Ann. Inst. Poincare 20, 69 (1974).

[27] S. Deser and P. van Nieuwenhuizen, Phys. Rev. Lett. B50, 245 (1974).

[28] L. Smolin, Towards a background indpendent approach to M theory, hep-th/9808192.

[29] I.L. Buchbinder, S.D. Odintsov and I.L. Shapiro, Effective Action in Quantum Gravity (IOP Publishing, 1992).

[30] B. Zwiebach, Phys. Lett. B156, 315 (1985).

[31] D. J. Gross and J.H. Sloan, Nucl. Phys. B291, 41 (1987).

[32] A. Tseytlin, Phys. Lett. B176, 92 (1986).

[33] G.L. Cardoso, D. Lüst and T. Mohaupt, Nucl. Phys. B450, 115 (1995), hep-th/9412209.

[34] V. Kaplunovsky, Nucl. Phys. B307, 145 (1988), hep-th/9205070, (E.: ibid B382, 436 (1992)).

[35] L.J. Dixon, V.S. Kaplunovsky and J. Louis, Nucl. Phys. B355, 649 (1991).

[36] J. M. Bardeen, B. Carter and S. W. Hawking, Comm. Math. Phys. 31, 161 (1973).

[37] G. Gibbons, R. Kallosh and B. Kol, Phys. Rev. Lett. 77, 4992 (1996), hep-th/9607108.

[38] S.W. Hawking, Phys. Rev. Lett. 26, 1344 (1971).

[39] N.D. Birrel and P.C.W. Davies, Quantum Fields in Curved Space-Time (Cambridge University Press, 1982).

[40] S.W. Hawking, Comm. Math. Phys. 43, 199 (1974).

[41] R.M. Wald, Quantum Field Theory in Curved Spacetime and Black Hole Thermodynamics (University of Chicago Press, 1994).

[42] J. Bekenstein, Phys. Rev. D7, 2333 (1973).

[43] J. Bekenstein, Phys. Rev. D9, 3292 (1974).

[44] A. Ghosh and P. Mitra, Phys. Rev. Lett. 78, 1858 (1997).

[45] G. 't Hooft, Dimensional Reduction in Quantum Gravity, hep-th/9310006, 1993.

[46] L. Susskind, J. Math. Phys. 36, 6377 (1995).

[47] C.G. Callan and J.M. Maldacena, Phys. Lett. B472, 591 (1996).

[48] G.T. Horowitz and A. Strominger, Phys. Rev. Lett. 77, 2368 (1996).

[49] J. Breckenridge, R. Myers, A. Peet and C. Vafa, Phys. Lett. B381, 423 (1996).

[50] J. Maldacena and A. Strominger, Phys. Rev. Lett. 77, 428 (1996). 
[51] C. Johnson, R. Khuri and R. Myers, Phys. Lett. B378, 78 (1996).

[52] J. Maldacena, Adv. Theor. Math. Phys. 2, 231 (1998).

[53] S.S. Gubser, I.R. Klebanov and A.M. Polyakov, Phys. Lett. B428, 105 (1998).

[54] E. Witten, Adv. Theor. Math. Phys. 2, 253 (1998).

[55] O. Aharony, S.S. Gubser, J. Maldacena, H. Ooguri and Y. Oz, Large N Field Theories, String Theory and Gravity, hep-th/9905111, 1999.

[56] B. Carter, In: Black Holes, Eds. C. DeWitt and B.S. De Witt, Gordon and Breach, New York, 1973.

[57] L. Racz and R.M. Wald, Class. Quant. Grav. 9, 2643 (1992).

[58] L. Racz and R.M. Wald, Global Extensions of Spacetimes describing Asymptotic Final States of Black Holes, gr-qc/9507055.

[59] J. Lee and R.M. Wald, J. Math. Phys. 31, 725 (1990).

[60] R.M. Wald, J. Math. Phys. 31, 2378 (1990).

[61] T. Jacobson, G. Kang and R. Myers, Phys. Rev. D48, 6587 (1994), gr-qc/9312023.

[62] V. Iyer and R.M. Wald, Phys. Rev. D50, 846 (1994), gr-qc/9403028.

[63] T. Jacobson, G. Kang and R.C. Myers, Black Hole Entropy in Higher Curvature Gravity, gr-qc/9502009.

[64] G.L. Cardoso, B. de Wit and T. Mohaupt, Deviations from the Area Law for Supersymmetric Black Holes, hep-th/9904005.

[65] B. de Wit, Modifications of the Area Law and $N=2$ Supersymmetric Black Holes, hep-th/9906095.

[66] V. Iyer and R.M. Wald, Phys. Rev. D52, 4430 (1995), gr-qc/9503052.

[67] R.M. Wald, Black Holes and Thermodynamics, gr-qc/9702022.

[68] R.M. Wald, Gravitation, Thermodynamics and Quantum Theory, gr-qc/9901033.

[69] G.W. Gibbons and C.M. Hull, Phys. Lett. B109, 190 (1982).

[70] B. Bertotti, Phys. Rev. 116, 1331 (1959).

[71] I. Robinson, Bull. Acad. Polon. 7, 351 (1959).

[72] S.D. Majumdar, Phys. Rev. 37, 930 (1947).

[73] A. Papapetrou, Proc. R. Irish Acad. A51, 191 (1947).

[74] J.B. Hartle and S.W. Hawking, Comm. Math. Phys. 26, 87 (1972).

[75] R. Rajaraman, Solitons and Instantons (North-Holland, 1982).

[76] J. Wess and J. Bagger, Supersymmetry and Supergravity (Princeton University Press, 1992).

[77] L. Alvarez-Gaume and S.F. Hassan, Introduction to S-Duality in $N=2$ Supersymmetric Gauge Theories, hep-th/9701069.

[78] C. Teitelboim, Phys. Lett. B69, 240 (1977). 
[79] P.K. Townsend, M-Theory from its Superalgebra, hep-th/9712004.

[80] C.M. Hull and P.K. Townsend, Nucl. Phys. B438, 109 (1994), hep-th/9410167.

[81] E. Witten, Nucl. Phys. B443, 85 (1995).

[82] B. de Wit, J. W. van Holten and A. van Proeyen, Nucl. Phys B167, 186 (1980), (E: ibid. B172, 543 (1980)).

[83] B. de Wit and A. Van Proeyen, Nucl. Phys. B245, 89 (1984).

[84] S. Ferrara, M. Kaku , P.K. Townsend and P. van Nieuwenhuizen, Nucl. Phys. B129, 125 (1977).

[85] B. de Wit and J. van Holten, Nucl. Phys. B155, 530 (1979).

[86] E. Fradkin and M. Vasiliev, Phys. Lett. B85, 47 (1979).

[87] M. Müller, Phys. Lett. B172, 353 (1986).

[88] M. Müller, Nucl. Phys. B282, 557 (1987).

[89] G. Akemann, R. Grimm, M. Hasler and C. Herrmann, Class. Quant. Grav. 16, 1617 (1999), hep-th/9812026.

[90] P. Claus, B. de Wit, M. Faux, B. Kleijin, R. Siebelink and P. Termonia, Nucl. Phys. B512, 148 (1998), hep-th/9712203, hep-th/9712203.

[91] G.L. Cardoso, B. de Wit and T. Mohaupt, Phys. Lett. B451, 309 (1999), hep-th/9812082.

[92] B. Kleijn, New Couplings in $\mathcal{N}=2$ Supergravity, PhD thesis, Utrecht University, 1998, ISBN 90-393-1975-8.

[93] A. van Proeyen, Superconformal tensor calculus in $N=1$ and $N=2$ supergravity, in Supersymmetry and supergravity '83, edited by B. Milewski, Singapore, 1983, Karpac Winter School 1983, World Scientific.

[94] B. de Wit, Introduction to Supergravity, in Supersymmetry and Supergravity '84, edited by P. F. B. de Wit and P. van Nieuwenhuizen, Singapore, 1984, World Scientific.

[95] L. Castellani, R. D’Auria and S. Ferrara, Class. Quant. Grav. 7, 1767 (1990).

[96] R. D’Auria, S. Ferrara and P. Fre, Nucl. Phys. B359, 705 (1991).

[97] P. Fre, Lectures on Special Kähler Geometry and Electric-Magnetic Duality, hep-th/9512043.

[98] L. Andrianopoli, M. Bertolini, A. Ceresole, R. D'Auria, S. Ferrara, P. Fre and T. Magri, $N=2$ Supergravity and $N=2$ Super Yang-Mills Theory on General Scalar Manifolds, hep-th/9605032.

[99] R.N. Mohapatra, Unification and Supersymmetry (Springer, 1986).

[100] R. Haag, J. Lopuszanski and M. Sohnius, Nucl. Phys. B88, 257 (1975).

[101] B. de Wit, Nucl. Phys. B158, 189 (1979).

[102] M. de Roo, J.W. van Holten, B. de Wit and A. van Proeyen, Nucl. Phys. B173, 175 (1980).

[103] R.J. Firth and J.D. Jenkins, Nucl. Phys. B85, 525 (1975). 
[104] P. Fayet, Ncul. Phys. B113, 135 (1976).

[105] R. Grimm, M. Sohnius and J. Wess, Nucl. Phys. B133, 275 (1978).

[106] P. Breitenlohner and M.F. Sohnius, Nucl. Phys. B178, 151 (1980).

[107] A. Salam and J. Strathdee, Ncul. Phys. B97, 293 (1975).

[108] B. de Wit, J.W. van Holten and A. Van Proeyen, Phys. Lett. B95, 51 (1980).

[109] P. Breitenlohner and M.F. Sohnius, Nucl. Phys. B187, 409 (1981).

[110] E. Bergshoeff, M. de Roo and B. de Wit, Nucl. Phys. B182, 173 (1981).

[111] B. de Wit, $N=2$ Electric-Magnetic Duality in a Chiral Background, in Proceedings of the 29-th Symposium Ahrenshoop on the Theory of Elementary Particles, p. 191, Nucl. Phys. Proc. Suppl. 49, 1996.

[112] M.K. Gaillard and B. Zumino, Nucl. Phys. B193, 221 (1981).

[113] P. Dirac, Proc. R. Soc. A133, 60 (1931).

[114] J. Schwinger, Phys. Rev. 144, 1087 (1966).

[115] D. Zwanziger, Phys. Rev. 176, 1480, 1489 (1968).

[116] E. Witten, Phys. Lett. B86, 283 (1979).

[117] B. Craps, F. Roose, W. Troost and A. Van Proeyen, Nucl. Phys. B503, 565 (1997), hep-th/9703082.

[118] P. Binetruy, G. Girardi and R. Grimm, Supergravity Couplings: A Geometric Formulation, hep-th/0005225.

[119] A. Strominger, Comm. Math. Phys. 133, 163 (1990).

[120] A. Ceresole, R. D'Auria, S. Ferrara and A. Van Proeyen, Nucl. Phys. B444, 92 (1995), hep-th/9502072.

[121] S. Ferrara, R. Kallosh and A.Strominger, Phys. Rev. D52, 5412 (1995), hep-th/9508072.

[122] A. Strominger, Phys. Lett. B383, 39 (1996), hep-th/9602111.

[123] S. Ferrara and R. Kallosh, Phys. Rev. D54, 1514 (1996), hep-th/9602136.

[124] S. Ferrara and R. Kallosh, Phys. Rev. D54, 1525 (1996), hep-th/9603090.

[125] K. Behrndt, G.L. Cardoso, B. de Wit, D.Lüst, T.Mohaupt and W. Sabra, Phys. Lett. B429 (1998), hep-th/9801081.

[126] W.A. Sabra, Mod. Phys. Lett. A12, 2585 (1997), hep-th/9703101.

[127] W.A. Sabra, Nucl. Phys. B510, 247 (1998), hep-th/9704147.

[128] K. Behrndt, D. Lüst and W.A. Sabra, Nucl. Phys. B510, 264 (1998), hep-th/9705169.

[129] K.P. Tod, Phys. Lett. B121, 241 (1983).

[130] B. Biran, F. Englert, B. de Wit and H. Nicolai, Phys. Lett. B124, 45 (1983).

[131] K. Behrndt, G.L. Cardoso, B. de Wit, R. Kallosh, D. Lüst and T. Mohaupt, Nucl. Phys. B488, 236 (1997), hep-th/9610105.

[132] S. Ferrara, G.W. Gibbons and R. Kallosh, Nucl. Phys. B500, 75 (1997), hep-th/9702103. 
[133] G. Moore, Arithmetic and Attractors, hep-th/9807087.

[134] R. Kallosh, A. Linde and M. Shmakova, Supersymmetric Multi Basin Attractors, hep-th/9910021.

[135] R. Kallosh, Multivalued Entropy of Supersymmetric Black Holes, hep-th/9912053.

[136] A. Chamseddine, S. Ferrara, G.W. Gibbons and R. Kallosh, Phys. Rev. D55, 3647 (1996), hep-th/9610155.

[137] L. Andrianopoli, R. D'Auria and S. Ferrara, Int. J. Mod. Phys. A12, 3759 (1997), hep-th/9608015.

[138] R. D'Auria and P. Fre, BPS Black Holes in Supergravity: Duality Groups, P-branes, Central Charges and the Entropy, in The Physics of Black Holes, 1998, hep-th/9812160.

[139] K. Behrndt, Phys. Lett. B396, 77 (1997), hep-th/9610232.

[140] R. Kallosh, M. Shmakova and W.K. Wong, Phys.Rev. D54, 6284 (1996), hep-th/9607077.

[141] B. de Wit and A. Van Proeyen, Phys. Lett. B293, 94 (1992), hep-th/9207091.

[142] W.A. Sabra, Mod. Phys. Lett. A13, 239 (1998).

[143] A.H. Chamseddine and W.A. Sabra, Phys. Lett. B426, 36 (1998).

[144] A.H. Chamseddine and W.A. Sabra, Phys. Lett. B460, 63 (1999), hep-th/9903046.

[145] A. Chou, R. Kallosh, J. Rahmfeld, S.-J. Rey, M. Shmakova and W.K. Wong, Nucl. Phys. B508, 147 (1997), hep-th/9704142.

[146] I. Gaida, S. Mahapatra, T. Mohaupt and W.A. Sabra, Class. Quant. Grav. 16, 419 (1999), hep-th/9807014.

[147] K. Behrndt, I. Gaida, D. Lüst, S. Mahapatra and T. Mohaupt, Nucl. Phys. B508, 659 (1997), hep-th/9706096.

[148] M. Bertolini and M. Trigiante, Regular R-R and NS-NS BPS black holes, hep-th/9910237.

[149] M. Bertolini and M. Trigiante, Regular BPS black holes: macroscopic and microscopic description of the generating solution, hep-th/0002191.

[150] J. Gutowski and G. Papadopoulos, Phys. Lett. B472, 45 (2000), hep-th/9910022.

[151] A. Maloney, M. Spradlin and A. Strominger, Superconformal Multi-Black Hole Moduli Spaces in Four Dimensions, hep-th/9911001.

[152] J. Gutowski and G. Papadopoulos, Phys. Rev. D62, 064023 (2000), hep-th/0002242.

[153] G.L. Cardoso, B. de Wit, J. Käppeli and T. Mohaupt, in preparation.

[154] R. Britto-Pacumio, J. Michelson, A. Strominger and A. Volovich, Lectures on Superconformal Quantum Mechanics and Multiblack Hole Moduli Spaces, hep-th/9911066, 1999 .

[155] P. Candelas, Lectures on Complex Manifolds, in Superstrings '87, 1987.

[156] T. Hübsch, Calabi-Yau Manifolds (World Scientific, 1992).

[157] B.R. Greene, String Theory on Calabi-Yau Manifolds, hep-th/9702155. 
[158] P. S. Aspinwall, Compactification, Geometry and Duality: $N=2$, hep-th/001001.

[159] J. Bagger and E. Witten, Ncul. Phys. B222, 1 (1983).

[160] P. Candelas, X. de la Ossa, P. Green and L. Parkas, Nucl. Phys. B359, 21 (1991).

[161] A. Strominger, Nucl. Phys. B451, 96 (1995), hep-th/9504090.

[162] B.R. Greene, D.R. Morrison and A. Strominger, Nucl. Phys. B451, 109 (1995), hep-th/9504145.

[163] W. Lerche, C. Vafa and N. Warner, Nucl. Phys. B324, 427 (1989).

[164] B. Greene and M. Plesser, Nucl. Phys. B338, 15 (1990).

[165] S. Hosono, A. Klemm and S. Theisen, Lectures on Mirror Manifolds, in Integrable Models and Strings, 1994, 3rd Baltic Student Seminar, Helsinki.

[166] I. Antoniadis, E. Gava, N.S. Narain and T.R. Taylor, Nucl. Phys. B413, 162 (1994).

[167] M. Bershadsky, S. Cecotti, H. Ooguri and C. Vafa, Comm. Math. Phys. 165, 311 (1993).

[168] S. Hosono, A. Klemm, S. Theisen and S.T. Yau, Comm. Math. Phys. 167, 301 (1995), hep-th/9308122.

[169] M. Bershadsky, S. Cecotti, H. Ooguri and C. Vafa, Nucl. Phys. B405, 279 (1993).

[170] S. Cecotti, P. Fendley, K. Intrilligator and C. Vafa, Nucl.Phys. B386, 405 (1992), hep-th/9204102.

[171] I. Antoniadis, E. Gava and K.S. Narain, Nucl. Phys. B383, 93 (1992), hep-th/9204030.

[172] M.A. Shifman and A.I. Vainshtein, Nucl. Phys. B 277, 456 (1986).

[173] V. Kaplunovsky and J. Louis, Nucl. Phys. B422, 57 (1994).

[174] V. Kaplunovsky and J. Louis, Nucl. Phys. B444, 191 (1995).

[175] R. Gopakumar and C. Vafa, M-Theory and Topological Strings-I, hep-th/9809187.

[176] R. Gopakumar and C. Vafa, M-Theory and Topological Strings-II, hep-th/9812127.

[177] A.C. Cadavid, A. Ceresole, R. D'Auria and S. Ferrara, Phys. Lett. B357, 76 (1995), hep-th/9506144.

[178] M. Günaydin, G. Sierra and P.K. Townsend, Nucl. Phys. B242, 244 (1984).

[179] M. Günaydin, G. Sierra and P.K. Townsend, Nucl. Phys. B253, 573 (1985).

[180] E. Witten, Nucl. Phys. B460, 195 (1996), hep-th/9603150.

[181] P.S. Aspinwall, K3 Surfaces and String Duality, hep-th/9611137.

[182] D.R. Morrison and C. Vafa, Nucl. Phys. B473, 74 (1996), hep-th/9602114.

[183] D.R. Morrison and C. Vafa, Nucl. Phys. B476, 437 (1996), hep-th/9603161.

[184] J. Louis, J. Sonnenschein, S. Theisen and S. Yankielowicz, Nucl. Phys. B480, 185 (1996), hep-th/9606049.

[185] S. Kachru and C. Vafa, Nucl. Phys. B450, 69 (1995), hep-th/9505105.

[186] S. Ferrara and A. Van Proeyen, Class. Quant. Grav. 6, 243 (1989).

[187] A. Giveon, M. Porrati and E. Rabinovici, Phys. Rept. 244, 77 (1994), hep-th/9401139. 
[188] B. de Wit, V. Kaplunovski, J. Louis and D. Lüst, Nucl. Phys. B451, 53 (1995), hep-th/9504006.

[189] A. Klemm, W. Lerche and P. Mayr, Phys. Lett. B357, 313 (1995), hep-th/9506112.

[190] I. Antoniadis and H. Partouche, Nucl. Phys. B460, 470 (1996), hep-th/9509009.

[191] G.L. Cardoso, G. Curio, D. Lüst and T. Mohaupt, Phys. Lett. B382, 241 (1996), hep-th/9603108.

[192] M.J. Duff, J.T. Liu and J. Rahmfeld, Nucl. Phys. B459, 125 (1996), hep-th/9508094.

[193] S. Ferrara, J.A. Harvey, A. Strominger and C. Vafa, Phys. Lett. B361, 59 (1995), hep-th/9505162.

[194] I. Antoniadis, S. Ferrara, E. Gava, K.S. Narain and T.R. Taylor, Nucl. Phys. B447, 35 (1995), hep-th/9504034.

[195] J.A. Harvey and G. Moore, Nucl. Phys. B463, 315 (1996), hep-th/9510182.

[196] I. Antoniadis, S. Ferrara and T.R. Taylor, Nucl. Phys. B460, 489 (1996), hep-th/9511108.

[197] C. Vafa and E. Witten, Dual String Pairs with $N=1$ and $N=2$ Supersymmetry in Four Dimensions, in S-Duality and Mirror Symmetry, 1995, Nucl. Phys. B Proc. Suppl. 46225 , hep-th/9507050.

[198] P.S. Aspinwall and J. Louis, Phys. Lett. B369, 233 (1996), hep-th/9510234.

[199] S. Kachru, A. Klemm, W. Lerche, P. Mayr and C. Vafa, Nucl. Phys. B459, 537 (1995), hep-th/9508155.

[200] N. Seiberg and E. Witten, Nucl. Phys. B426, 19 (1994), hep-th/9407087.

[201] A. Klemm, On the Geometry behind $N=2$ Supersymmetric Effective Actions in Four Dimensions, in Duality - Strings and Fields, 1997, 33rd Karpacz Winter School of Theoretical Physics, hep-th/9705131.

[202] P. Mayr, Fortsch. Phys. 47, 39 (1999).

[203] P.S. Aspinwall and M.R. Plesser, T-Duality Can Fail, hep-th/9905036, 1999.

[204] W. Lerche, D.-J. Smit and N.P. Warner, Nucl. Phys. B372, 87 (1992), hep-th/9108013.

[205] A. Ceresole, R. D’Auria and T. Regge, Nucl. Phys. B414, 517 (1994), hep-th/9307151.

[206] V. Kaplunovsky, J. Louis and S. Theisen, Phys. Lett. B357, 71 (1995), hep-th/9506110.

[207] M. Marino and G. Moore, Nucl. Phys. B543, 592 (1999), hep-th/9808131.

[208] G.L. Cardoso, G. Curio, D. Lüst, T. Mohaupt and S.-J. Rey, Nucl. Phys. B464, 18 (1996), hep-th/9512129.

[209] G. Curio, Phys. Lett. B366, 131 (1996), hep-th/9509042.

[210] G. Curio, Phys. Lett. B368, 78 (1996), hep-th/9509146.

[211] I. Antoniadis, E. Gava, K.S. Narain and T.R. Taylor, Nucl. Phys. B455, 109 (1995), hep-th/9507115.

[212] B. de Wit, G.L. Cardoso, D. Lüst, T. Mohaupt and S.-J. Rey, Nucl. Phys. B481, 353 (1996), hep-th/9607184, hep-th/9607184. 
[213] M. de Roo, Nucl. Phys. B255, 515 (1985).

[214] A. Sen, Int. Jour. Mod. Phys. A329, 217 (1994), hep-th/9402032.

[215] J.A. Harvey and G. Moore, Phys. Rev. D57, 2323 (1998), hep-th/9610237.

[216] A. Sen, Nucl. Phys. B450, 103 (1995), hep-th/9504027.

[217] J.A. Harvey and A. Strominger, Nucl. Phys. B449, 535 (1995), hep-th/9504047.

[218] J.A. Harvey and G. Moore, Phys. Rev. D57, 2329 (1998), hep-th/9611176.

[219] J. Maldacena, A. Strominger and E. Witten, JHEP 2 (1997), hep-th/9711053.

[220] C. Vafa, Adv. Theor. Math. Phys. 2, 207 (1998), hep-th/9711067.

[221] R. Kallosh and B. Kol, Phys. Rev. D53, 5344 (1996), hep-th/9602014.

[222] M. Cvetic and D. Youm, Phys. Rev. D53, 584 (1996), hep-th/9507090.

[223] M. Cvetic and A.A. Tseytlin, Phys. Rev. D53, 5619 (1996), hep-th/952103.

[224] G.L. Cardoso, B. de Wit and T. Mohaupt, Nucl. Phys. 567B, 87 (2000), hep-th/9906094.

[225] M. Shmakova, Phys. Rev. D56, 540 (1997), hep-th/9612076.

[226] C.T.C. Wall, Invent. Math. 1, 355 (1966).

[227] K. Behrndt, R. Kallosh, J. Rahmfeld, M. Shmakova and W.K. Wong, Phys. Rev. D54, 6293 (1996), hep-th/9608059.

[228] G.L. Cardoso, D. Lüst and T. Mohaupt, Phys. Lett. B388, 266 (1996), hep-th/9608099.

[229] S.-J. Rey, Nucl. Phys. B508, 569 (1997), hep-th/9610157.

[230] K. Behrndt and I. Gaida, Phys. Lett. B401, 263 (1997), hep-th/9702168.

[231] K. Behrndt, G.L. Cardoso and I. Gaida, Nucl. Phys. B506, 267 (1997), hep-th/9704095.

[232] K. Behrndt and T. Mohaupt, Phys. Rev. D56, 2206 (1997), hep-th/9611140.

[233] K. Stelle, Lectures on Supergravity p-Branes, in High Energy Physics and Cosmology, p. 287, 1996, hep-th/9701088.

[234] A.A. Tseytlin, Nucl. Phys. B475, 149 (1996), hep-th/9604035.

[235] A.A. Tseytlin, Nucl. Phys. B475, 431 (1996), hep-th/9605091.

[236] K. Becker, M. Becker and A. Strominger, Nucl. Phys. B456, 130 (1995), hep-th/9507158.

[237] D. Freed, J.A. Harvey, R. Minasian and G. Moore, Adv. Theor. Math. Phys. 2, 601 (1998), hep-th/9803205, hep-th/9803205.

[238] F. Larsen and F. Wilczek, Phys. Lett. B375, 37 (1996), hep-th/9511064.

[239] T. Banks, W. Fischler, S.H. Shenker and L. Susskind, Phys. Rev. D55, 5112 (1996), hep-th/9610043.

[240] G.W. Gibbons and P.K. Townsend, Phys. Lett. B454, 187 (1999), hep-th/9812034.

[241] J. Michelson and A. Strominger, Superconformal Multi-Black Hole Quantum Mechanics, hep-th/9908044.

[242] T. Banks, W. Fischler, I.R. Klebanov and L. Susskind, Phys. Rev. Lett. 80, 226 (1998), hep-th/9709091. 
[243] I.R. Klebanov and L. Susskind, Phys. Lett. B416, 62 (1998), hep-th/9707108.

[244] T. Banks, W. Fischler, I.R. Klebanov and L. Susskind, JHEP 9801 (1998), hep-th/9711005.

[245] J. Schwarz, TASI Lectures on Non-BPS D-Branes Systems, hep-th/9908144.

[246] I.R. Klebanov and A.A. Tseytlin, Nucl. Phys. B546, 155 (1999), hep-th/9811035.

[247] I. Bakas, M. Bourdeau and G.L. Cardoso, Nucl. Phys. B510, 103 (1998), hep-th/9706032.

[248] S. Chandrasekhar, The Mathematical Theory of Black Holes (Oxford Science Publications, 1992).

[249] M. Cvetic, A. Font, L.E. Ibanez, D. Lüst and F. Quevedo, Nucl. Phys. B361, 194 (1991).

[250] J. Erler, Investigation of Moduli Spaces in String Theory, PhD thesis, TU München, 1992, MPI-PH-92-21. 